\title{
Cryopreservation of pancreatic islets
}

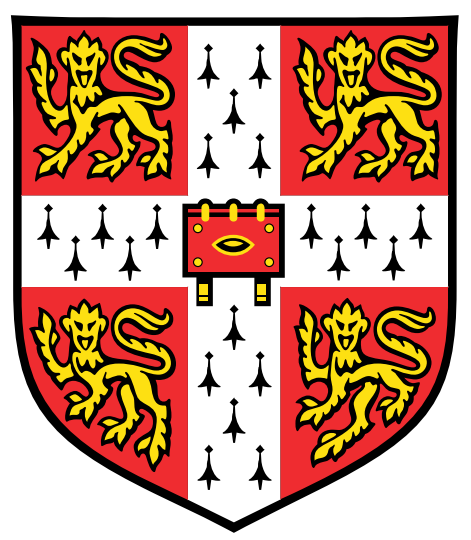

Nikola Doležalová

Supervisors: Dr. Kourosh Saeb-Parsy, Prof. Nigel K. H. Slater

Department of Surgery

Department of Chemical Engineering and Biotechnology

University of Cambridge

This dissertation is submitted for the degree of

Doctor of Philosophy

Hughes Hall

February 2019 



\section{Declaration}

This dissertation is the result of my own work and includes nothing which is the outcome of work done in collaboration except as declared in the Acknowledgements and specified in the text. It is not substantially the same as any that I have submitted, or, is being concurrently submitted for a degree or diploma or other qualification at the University of Cambridge or any other University or similar institution. I further state that no substantial part of my dissertation has already been submitted, or, is being concurrently submitted for any such degree, diploma or other qualification at the University of Cambridge or any other University or similar institution. This dissertation contains fewer than 60,000 words excluding figures, photographs, tables, appendices and bibliography.

Nikola Doležalová

February 2019 



\title{
Cryopreservation of pancreatic islets
}

\author{
Nikola Doležalová
}

Pancreatic islet transplantation is a curative treatment for diabetes but access to this therapy is limited by the availability of islets. Long-term cryopreservation of islets could partially address this limitation but current cryopreservation protocols, which use the toxic cryoprotectant dimethyl sulphoxide (DMSO), achieve suboptimal cryosurvival and are not clinically used. The aim of this project was to develop an improved and clinically-relevant method for cryopreservation of islets. The project examined the efficacy of the non-toxic cryoprotectant trehalose in combination with (1) membrane-permeabilising biopolymers PP-50 and PP-75 to increase intracellular uptake, and (2) antioxidants MitoQ and salidroside to reduce cryopreservation-induced oxidative stress.

Due to the complex architecture of pancreatic islets, human mononuclear cells were first used to optimise the use of permeabilising polymers. Current methods of viability assessment were critically examined and improved, followed by systematic screening of cryopreservation conditions. The efficacy of promising cryopreservation protocols were subsequently confirmed for mouse pancreatic islets using viability and functional assays.

PP-50 and PP-75 polymers were shown to be unsuitable for trehalose-based cryopreservation of mononuclear cells and islets due to potential toxicity and need for long incubation. Investigation of diffusion kinetics demonstrated that a minimal incubation time of $6 \mathrm{~h}$ at $37^{\circ} \mathrm{C}$ is required to enable diffusion of solutes into the islet core. This finding implies that current clinical viability assessment protocols only survey the periphery of islets. A range of imaging modalities were used to confirm that islet core viability can be dramatically different from the islet periphery. Using these insights, a novel cryopreservation protocol was developed in which islets are pre-incubated for $6 \mathrm{~h}$ at $37^{\circ} \mathrm{C}$ with trehalose and antioxidants, followed by addition of DMSO, resulting in significantly greater viability of cryopreserved islets.

In conclusion, this study identified important limitations of the current cryobiological viability assessment of mononuclear cells and pancreatic islets. Permeabilising biopolymers did not improve trehalose-based cryopreservation in primary cells. An improved method for cryopreservation of pancreatic islets was developed which combines DMSO and trehalose in a novel manner and can be further improved by addition of antioxidants. 



\section{Acknowledgements}

I would like to thank to my supervisor Dr. Kourosh Saeb-Parsy for his support and guidance throughout this project. Thank you for dedicating plenty of time and effort to help me on the way to become a researcher. Thank you for teaching me that every problem has a solution and that the only way to fail is not to try. I cannot imagine a more inspiring, caring (and fun) $\mathrm{PhD}$ supervisor.

I would also like to thank my other supervisor, Prof. Nigel K. H. Slater, for his undying positivity and for excellent advice. Thank you for providing the chemical engineering expertise whenever my biology background would not suffice.

Very special gratitude goes to my science family, the KSP group. Thank you, Timothy Elliott Beach, Anja Gruszczyk, Nikitas Georgakopoulos, Olivia Tysoe, Alba Matas Cespedes, Krishnaa Mahbubani, Michelle Curran and Bethany Bareham for being wonderful colleagues but even better friends. I feel very blessed to have met these people and proud to be able to work with them.

My research would not be possible without the help and support of all colleagues from the Department of Surgery. I would especially like to thank Sylvia Rehakova, Fae Hills, Rachel Seear, Margaret C. Negus, Paul D. Smith, Lila Tran, Alison Warrington, Linda Butler and Ivonne Gamper for brilliant technical support. Thank you to all other colleagues from the department for making it a great place to work. I would also like to thank colleagues from the Department of Chemical Engineering and Biotechnology for providing expertise about the permeabilising polymers and antioxidants.

I am very grateful to my first year viva examiners, Dr. Graham Christie and Dr. Thomas Moreau, as well as to my colleague Noha Al-Otaibi for providing useful advice on the direction of the project.

I am very grateful to the W. D. Armstrong Fund for providing me with PhD studentship and thus making this project possible.

I would also like to acknowledge those who helped with writing and corrections of this thesis, especially to Kourosh Saeb-Parsy, Nigel K. H. Slater and Vincentius Aji Jatikusumo.

Thank you to the Cambridge Biorepository for Translational Medicine (CBTM) for obtaining and processing human tissue, especially to Dr. Krishnaa Mahbubani, Bethany 
Bareham, Nikitas Georgakopoulos, Olivia Tysoe and Kathleen Elliott. I am also very grateful to Mr. John Casey and the SNBTS's Islet Isolation Laboratory in Edinburgh isolation and provision of human pancreatic islets for research and their advice. I am very grateful to all human donors and their families for providing the gift of human tissue for research. Without them this project would not be possible.

I would especially like to thank all collaborators who contributed to this project:

- to Anja Gruszczyk and Prof. Mike Murphy from the MRC Mitochondrial Biology Unit, University of Cambridge, for their help with the antioxidant experiments;

- to Dr. Kerry Barkan and Dr. Graham Ladds from the Department of Pharmacology, University of Cambridge, for their help with cAMP release assays;

- to Dr. Jakub Surmacki and Dr. Sarah E. Bohndiek from the Cavendish Laboratory, University of Cambridge, for their help with Raman spectroscopy experiments;

- to Jackie Higgins from the Department of Surgery for providing excellent care for my experimental animals and helping with animal procedures;

- to the staff of Central Biomedical Services for housing and maintaining the experimental animals and providing support for the in vivo experimental work;

- to Dr. Elisa Laurenti and Emily Calderbank from the Stem Cell Institute, University of Cambridge, for their help with CFU assays;

- to Dr. Brijesh Yadav and Alexandra Polakova who were visiting student at the Department of Surgery for helping with some of the splenocyte experiments;

- to Dr. Krishnaa Mahbubani and Dr. Alex Chen for synthesising and providing the permeabilising polymers and the proton nuclear magnetic resonance data;

- to Francesco Pampaloni and Till Moreth from the Goethe University in Frankfurt for their help with light sheet microscopy imaging;

- to Kevin O'Holleran and Martin Oliver Lenz from the Cambridge Advanced Imaging Centre, University of Camrbidge, for help with multi-photon imaging;

- to Core Biochemical Assay Laboratory (CBAL) for biochemistry measurements;

- to BRC Phenotyping Hub for providing training and support for confocal microscopy and flow cytometry. 
I am grateful to my family and my dearest friends Jana Stupková, Miroslav Vojtek, Soňa Večeřová and Hana Zavadilová who always believe in me and send me love from far away. Also thank you to Veronika Kolejáková who encouraged me to aim high. Without her I would never think of applying to Cambridge.

Thank you to the love of my life, Aji, for always standing by me, always having words of encouragement and for making me feel at home, wherever I am.

Most of all, I am grateful to my loving parents and my little sister who never doubted me even when I did, who have always been there when things were not going well and who are a great inspiration for my life. I want to dedicate this thesis to them. 



\section{Conference presentations}

53 $^{\text {rd }}$ Annual Meeting of the Society for Cryobiology (July 2016 in Ottawa, Canada)

- Oral presentation: A novel cryoprotectant for preservation of human haematopoietic stem cells

- Abstract published: Cryobiology, Volume 73, Pages 400-401 (December 2016)

54 ${ }^{\text {th }}$ Annual Meeting of the Society for Cryobiology (July 2017 in Hefei, China)

- Work shortlisted for the Peter Steponkus Crystal Award

- Oral presentation: Trehalose and membrane-permeabilising biopolymers PP-50 and PP-75 for cryopreservation of pancreatic islets

- Abstract published: Cryobiology, Volume 80, Pages 175-176 (February 2018)

$\mathbf{1 8}^{\text {th }}$ Congress of the European Society for Organ Transplantation (September 2017 in Barcelona, Spain)

- Oral presentation: DMSO-free cryopreservation of pancreatic islets using trehalose and membrane-permeabilising biopolymer PP-50

$2^{\text {st }}$ British Transplantation Society Annual Congress (March 2018 in Brighton, UK)

- Work shortlisted for the Medawar Medal

- Oral presentation: Accurate viability assessment and cryopreservation of pancreatic islets requires prolonged incubation with viability dyes and cryoprotectants for more than 12 hours

55 $^{\text {th }}$ Annual Meeting of the Society for Cryobiology (July 2018 in Madrid, Spain)

- Work shortlisted for the Peter Steponkus Crystal Award

- Oral presentation: Characterizing diffusion kinetics to improve pancreatic islet cryopreservation

- Abstract published: Cryobiology, Volume 85, Pages 144-145 (December 2018) 



\section{Manuscripts in preparation}

Manuscript 1: Pre-incubation with trehalose improves DMSO-based cryopreservation of mouse pancreatic islets

Manuscript 2: Standardisation of viability assessment methods in cryobiology

Manuscript 3: Current methods of staining and imaging of pancreatic islet viability disregards islet core 



\begin{abstract}
Pancreatic islet transplantation is a curative treatment for diabetes but access to this therapy is limited by the availability of islets. Long-term cryopreservation of islets could partially address this limitation but current cryopreservation protocols, which use the toxic cryoprotectant dimethyl sulphoxide (DMSO), achieve suboptimal cryosurvival and are not clinically used. The aim of this project was to develop an improved and clinically-relevant method for cryopreservation of islets. The project examined the efficacy of the non-toxic cryoprotectant trehalose in combination with (1) membrane-permeabilising biopolymers PP-50 and PP-75 to increase intracellular uptake, and (2) antioxidants MitoQ and salidroside to reduce cryopreservation-induced oxidative stress.

Due to the complex architecture of pancreatic islets, human mononuclear cells were first used to optimise the use of permeabilising polymers. Current methods of viability assessment were critically examined and improved, followed by systematic screening of cryopreservation conditions. The efficacy of promising cryopreservation protocols were subsequently confirmed for mouse pancreatic islets using viability and functional assays.

PP-50 and PP-75 polymers were shown to be unsuitable for trehalose-based cryopreservation of mononuclear cells and islets due to potential toxicity and need for long incubation. Investigation of diffusion kinetics demonstrated that a minimal incubation time of $6 \mathrm{~h}$ at $37^{\circ} \mathrm{C}$ is required to enable diffusion of solutes into the islet core. This finding implies that current clinical viability assessment protocols only survey the periphery of islets. A range of imaging modalities were used to confirm that islet core viability can be dramatically different from the islet periphery. Using these insights, a novel cryopreservation protocol was developed in which islets are pre-incubated for $6 \mathrm{~h}$ at $37{ }^{\circ} \mathrm{C}$ with trehalose and antioxidants, followed by addition of DMSO, resulting in significantly greater viability of cryopreserved islets.

In conclusion, this study identified important limitations of the current cryobiological viability assessment of mononuclear cells and pancreatic islets. Permeabilising biopolymers did not improve trehalose-based cryopreservation in primary cells. An improved method for cryopreservation of pancreatic islets was developed which combines DMSO and trehalose in a novel manner and can be further improved by addition of antioxidants.
\end{abstract}





\section{Table of Contents}

$\begin{array}{ll}\text { List of Figures } & \text { xxi }\end{array}$

$\begin{array}{ll}\text { List of Tables } & \mathbf{x x v}\end{array}$

List of Abbreviations $\quad$ xxvii

1 Introduction 1

1.1 Need for cryopreservation of cells and tissues . . . . . . . . . . . . 1

1.2 Pancreatic islets ...................... 2

1.2.1 Pancreatic islet morphology and function . . . . . . . . . . 2

1.2.2 Regulation of insulin secretion in $\beta$-cells . . . . . . . . . . 2

1.2.3 Regulation of blood glucose levels . . . . . . . . . . . . . 3

1.2.4 Pancreatic islets in diabetes treatment . . . . . . . . . . . 5

1.2.5 Cryopreservation in pancreatic islet transplantation . . . . . . . 6

1.3 Mononuclear cells . . . . . . . . . . . . . . . 7

1.3.1 Sources and use of mononuclear cells . . . . . . . . . . . 7

1.3.2 Haematopoietic progenitors and their cryopreservation . . . . . . 7

1.4 Physical aspects of freezing cells . . . . . . . . . . . . . . 9

1.4.1 Overview of freezing in biological systems . . . . . . . . . . 9

1.4.2 Theories and mechanism of cryoinjury . . . . . . . . . . . 9

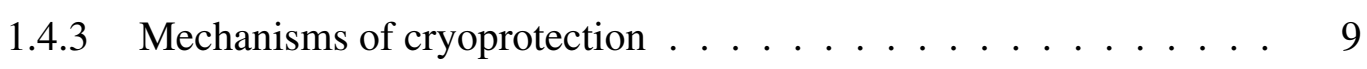

1.5 Cryoprotectants . . . . . . . . . . . . . . . . . . . 10

1.5.1 The ideal cryoprotectant . . . . . . . . . . . . . . . . . . . . 10

1.5.2 Overview of cryoprotectants . . . . . . . . . . . . 11

1.5.3 Mechanisms of cryoprotectant toxicity . . . . . . . . . . . . 12

1.6 Current cryopreservation methods . . . . . . . . . . . . . . 12

1.6.1 HSC cryopreservation protocols . . . . . . . . . . . . . 12

1.6.2 Pancreatic islet cryopreservation protocols . . . . . . . . . . . . 14 
1.7 Current methods of cryosurvival assessment . . . . . . . . . . . . . . 18

1.7.1 Assessment of mononuclear cell cryosurvival . . . . . . . . . . . 18

1.7.2 Assessment of pancreatic islet cryosurvival . . . . . . . . . . 19

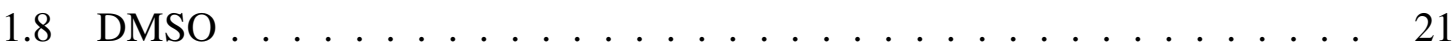

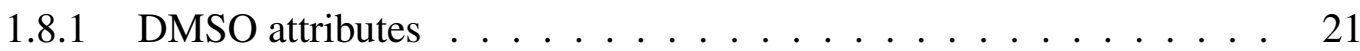

1.8.2 DMSO toxicity . . . . . . . . . . . . . 22

1.8 .3 Alternatives to DMSO . . . . . . . . . . . . . 22

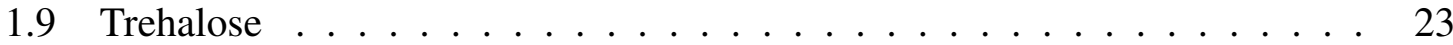

1.9.1 Trehalose attributes . . . . . . . . . . . . . 23

1.9.2 Cryopreservation with trehalose . . . . . . . . . . . . 24

1.9.3 Intracellular trehalose delivery . . . . . . . . . . . . . . . 24

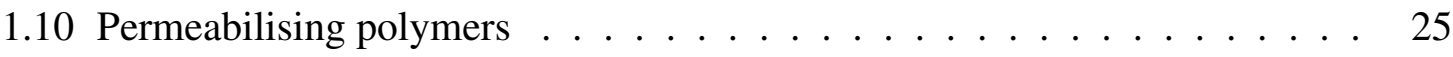

1.10.1 Characteristics . . . . . . . . . . . . . . 25

1.10 .2 PP-50 efficacy and toxicity . . . . . . . . . . . 26

1.10.3 Trehalose and permeabilising polymers . . . . . . . . . . 27

1.11 Antioxidants . . . . . . . . . . . . . . . . . . 27

1.11.1 Oxidative stress in cryopreservation . . . . . . . . . . . . 27

1.11 .2 Salidroside . . . . . . . . . . . . . . . 28

1.11 .3 MitoQ . . . . . . . . . . . . . . . . . . . 29

1.12 Project aims . . . . . . . . . . . . . . . . 30

1.13 Experimental approach . . . . . . . . . . . . . . 31

2 Materials and Methods $\quad 33$

2.1 Tissues from deceased human organ donors . . . . . . . . . . . . . 33

2.1 .1 Tissue retrieval . . . . . . . . . . . . . . . . . 33

2.1 .2 Processing tissues . . . . . . . . . . . . 33

2.1.3 Obtaining human pancreatic islets . . . . . . . . . . 34

2.1 .4 Information storage . . . . . . . . . . . . . . . . 34

2.2 Animals and animal procedures . . . . . . . . . . . . . . . . . . . . . . . . . 35

2.2.1 Animal provenance and husbandry . . . . . . . . . . . . . 35

2.2.2 Isolation of mouse pancreatic islets . . . . . . . . . . . . 35

2.2.3 Transplantation of islets under the kidney capsule of NSG mice . . 36

2.2.4 Induction of diabetes in NSG mice . . . . . . . . . . . . . . . 37

2.2.5 Collection and processing of mouse peripheral blood . . . . . . . 38

2.3 Compound provision and synthesis . . . . . . . . . . . . . . 39

2.3.1 Synthesis of PP-50 and PP-75 . . . . . . . . . . . . . . . 39

2.3.2 Fluorescent labelling of PP-50 . . . . . . . . . . . . . 40 
2.3.3 Provision of antioxidants . . . . . . . . . . . . . . 41

2.4 Cryopreservation . . . . . . . . . . . . . . . . . . 41

2.4.1 Cryopreservation of mononuclear cells . . . . . . . . . . . . 41

2.4.2 Thawing of mononuclear cells . . . . . . . . . . . . . . . 41

2.4.3 Cryopreservation of pancreatic islets . . . . . . . . . . . . . 42

2.4.4 Thawing of pancreatic islets . . . . . . . . . . . . . 43

2.4 .5 Liquid nitrogen storage . . . . . . . . . . . . . . . . . 44

2.5 Assays for single cell analysis . . . . . . . . . . . . . . . . . 44

2.5.1 Cell counting . . . . . . . . . . . . . . . . . 44 44

2.5.2 Toxicity assays for mononuclear cells . . . . . . . . . . . . 45

2.5.3 Culture assays for mononuclear cells . . . . . . . . . . . . 45

2.5.4 Flow cytometry of mononuclear cells . . . . . . . . . . . . 45

2.5.5 Fluorescence activated cell sorting (FACS) . . . . . . . . . . 46

2.5.6 Haematopoietic colony forming unit (CFU) assay . . . . . . . . . . 47

2.5 .7 Total protein assay . . . . . . . . . . . . . . . . . . . . . . . . . . . . . . .

2.6 Assays for pancreatic islets . . . . . . . . . . . . . . . . 50

2.6.1 Assessment of purity by dithizone staining . . . . . . . . . . 50

2.6.2 Assessment of viability by fluorescent imaging . . . . . . . . . 50

2.6.3 Assessment of viability by flow cytometry . . . . . . . . . . . . 52

2.6.4 Diffusion assays for pancreatic islets . . . . . . . . . . . . . 52

2.6.5 Toxicity assays for pancreatic islets . . . . . . . . . . 53

2.6 .6 ATP/ADP assay . . . . . . . . . . . . . . . . . 54

2.6 .7 Static glucose stimulation assay . . . . . . . . . . . . 55

2.6.8 Mitochondrial DNA oxidation assay . . . . . . . . . . . . 57

2.6 .9 Cyclic AMP release assay . . . . . . . . . . . . . . . 5 58

2.7 Imaging . . . . . . . . . . . . . . . . . . . 59

2.7.1 Confocal microscopy . . . . . . . . . . . . . . . . . . 59

2.7.2 Multi-photon microscopy . . . . . . . . . . . . . . . . 60

2.7.3 Light sheet fluorescence microscopy . . . . . . . . . . . . . . 60

2.7.4 Raman spectroscopy . . . . . . . . . . . . . . . . . . . 61

2.8 Statistical analysis . . . . . . . . . . . . . . . . . . . 62

$3 \quad$ Viability assessment of mononuclear cells and pancreatic islets 63

3.1 Introduction and aims . . . . . . . . . . . . . . . . . 63

3.2 Suitable viability assessment method for cryobiology . . . . . . . . . . . 64

3.3 Cryosurvival assessment for mononuclear cells . . . . . . . . . . . 66

3.3.1 Gating using light-scattering properties versus fluorescence . . . . . 66 
3.3.2 Quantification of viability and cryopreservation efficacy . . . . 68

3.3.3 Survival of cell subsets . . . . . . . . . . . . . . . . 70

3.4 Cryosurvival assessment for pancreatic islets . . . . . . . . . . . . 70

3.4.1 Current viability assessment method evaluation . . . . . . . . 70

3.4.2 Increasing diffusion rate of molecules into pancreatic islets . . . . . 73

3.4.3 Improvement of islet viability imaging method . . . . . . . . . . 78

3.5 Discussion . . . . . . . . . . . . . . . . . . . . . 80

3.5.1 Mononuclear cell viability assessment . . . . . . . . . . . . . 80

3.5.2 Pancreatic islet viability assessment . . . . . . . . . . . . 80

3.6 Conclusion . . . . . . . . . . . . . . . . . . . . 83

4 Splenocyte cryopreservation with trehalose and permeabilising polymers $\quad 85$

4.1 Introduction and aims . . . . . . . . . . . . . . . . . . . 85

4.2 Initial efficacy of trehalose and PP-50 . . . . . . . . . . . . . 86

4.2.1 Efficacy for cryopreservation of splenic mononuclear cells . . . . 86

4.2.2 Efficacy for cryopreservation of bone marrow mononuclear cells . . 88

4.2.3 Colony forming ability of cryopreserved CD34 ${ }^{+}$cells . . . . . . . . 91

4.3 General parameters of cryopreservation protocol . . . . . . . . . . . . . . 92

4.3 .1 Cell density . . . . . . . . . . . . . . . . . . 92

4.3.2 Addition of fetal calf serum . . . . . . . . . . . . . . . . . 94

4.3.3 Optimisation of thawing protocol . . . . . . . . . . . . . . 97

4.4 Incubation parameters for trehalose and PP-50 . . . . . . . . . . . . . 105

4.4.1 Incubation time at $37^{\circ} \mathrm{C} \ldots \ldots . \ldots 105$

4.4 .2 Incubation temperature . . . . . . . . . . . . . . 107

4.4 .3 Incubation $\mathrm{pH} \ldots \ldots \ldots \ldots 111$

4.5 Concentration of components . . . . . . . . . . . . . . . 113

4.5.1 Concentration of trehalose . . . . . . . . . . . . . 113

4.5.2 Concentration of polymers . . . . . . . . . . . . . 116

4.6 Function and safety of permeabilising polymers . . . . . . . . . . . . 118

4.6.1 Trehalose uptake measurements by Raman spectroscopy . . . . . . 118

4.6.2 Safety of permeabilising biopolymers . . . . . . . . . . . . . . 121

4.7 Discussion . . . . . . . . . . . . . . . . . . . . . 121

4.7.1 Trehalose as an alternative to DMSO . . . . . . . . . . . . 121

4.7.2 PP-50 as a method of intracellular trehalose delivery . . . . . . . 122

4.7.3 Safety of the new protocol . . . . . . . . . . . . . 123

4.8 Conclusion . . . . . . . . . . . . . . . . . . . . . . . . . . . . 124 
5 Developing a novel islet cryopreservation protocol 125

5.1 Introduction and aims . . . . . . . . . . . . . . . . . . 125

5.2 Toxicity of cryoprotectants for pancreatic islets . . . . . . . . . . . 126

5.2 .1 Toxicity of DMSO . . . . . . . . . . . . . . 126

5.2 .2 Toxicity of trehalose . . . . . . . . . . . . . . 128

5.3 Optimisation of DMSO protocol parameters . . . . . . . . . . . . . 128

5.3 .1 Addition of DMSO . . . . . . . . . . . . . . . . . . 128

5.3 .2 Freezing rate . . . . . . . . . . . . . . . . 132

5.3.3 DMSO removal protocol . . . . . . . . . . . . . . . 134

5.4 Trehalose as a sole cryoprotectant for islets . . . . . . . . . . . . . . 139

5.4 .1 Trehalose concentration . . . . . . . . . . . . . . 139

5.4 .2 Trehalose incubation time . . . . . . . . . . . . . 139

5.5 Addition of trehalose to DMSO protocol . . . . . . . . . . . . . . . . 142

5.5.1 Effect of pre-incubation with trehalose . . . . . . . . . . . . . 142

5.5.2 Concentrations of components . . . . . . . . . . . . 146

5.6 Addition of antioxidants: MitoQ and salidroside . . . . . . . . . . . . . 150

5.6.1 Effect of antioxidants during cryopreservation . . . . . . . . . 150

5.6.2 Stage of antioxidant addition . . . . . . . . . . . . . 152

5.7 Function of islets cryopreserved by new protocols . . . . . . . . . . . . . 154

5.7 .1 In vitro efficacy . . . . . . . . . . . . . . . . . . . . . . . . . . 154

5.7 .2 In vivo efficacy . . . . . . . . . . . . . . . . . . . . . . 157

5.8 Discussion . . . . . . . . . . . . . . . . . . . . . . . 160

5.8.1 Cryopreservation of islets with trehalose . . . . . . . . . . 160

5.8.2 New insight into cell cluster survival . . . . . . . . . . . . . . . 162

5.8 .3 Cryopreservation with antioxidants . . . . . . . . . . 162

5.9 Conclusion . . . . . . . . . . . . . . . . 163

6 General discussion $\quad 165$

6.1 Introduction . . . . . . . . . . . . . . . . . . 165

6.2 Research aims and major findings _. . . . . . . . . . . . . 165

6.3 Impact of the findings . . . . . . . . . . . . . . . . . . 166

6.3.1 Standards of viability assessment in cryobiology . . . . . . . 166

6.3.2 Limitations in pancreatic islet viability assessment . . . . . . . . 166

6.3.3 Diffusion of small molecules into the core of pancreatic islets . . . 167

6.3.4 Trehalose potential for cryopreservation of mononuclear cells . . . 168

6.3.5 Safety and efficacy of permeabilising polymers in primary cells . . 169

6.3.6 Novel cryopreservation protocol for pancreatic islets . . . . . . . 170 
6.3.7 Potential of antioxidants in islet cryopreservation . . . . . . . . 171

6.3.8 Cryopreservation of other multicellular aggregates . . . . . . . 171

6.4 Limitations . . . . . . . . . . . . . . . . . . . . 173

6.4.1 Assumptions for diffusion studies . . . . . . . . . . . 173

6.4.2 Unavailability of islet size data . . . . . . . . . . . . . . 173

6.4.3 Statistical analysis of the bi-modally distributed data . . . . . . . 174

6.4.4 Mouse islets as an experimental model . . . . . . . . . . . . 174

6.4.5 Short-term cryopreservation . . . . . . . . . . . . . 175

6.5 Future directions . . . . . . . . . . . . . . . . . . 175

6.5.1 Viability assessment . . . . . . . . . . . . 175

6.5 .2 Cell cluster survival _. . . . . . . . . . . . 176

6.5.3 Further modifications of the cryopreservation protocol . . . . . . 176

6.5.4 Validation of the novel cryopreservation protocol . . . . . . . . . 177

6.6 Conclusion . . . . . . . . . . . . . . . . . . . . 180

$\begin{array}{ll}\text { References } & 183\end{array}$

$\begin{array}{lll}\text { Appendix A Donor demographics } & \mathbf{2 0 3}\end{array}$

A.1 Demographic information of deceased human donors . . . . . . . . . . 203

A.2 Summary of demographic parameters for experimental sets . . . . . . . . . 204

A.3 Human islet preparation characteristics . . . . . . . . . . . . . . . . 205

$\begin{array}{lll}\text { Appendix B } & \text { Flow cytometry panels and gating strategy } & 207\end{array}$

Appendix C Efficacy of permeabilising polymers in islet cryopreservation 211

C.1 Toxicity of polymers for pancreatic islets . . . . . . . . . . . . . . 211

C.2 Polymer attachment to cell membrane . . . . . . . . . . . . . . . 213

C.3 Polymer efficacy in cryopreservation . . . . . . . . . . . . 215

Appendix D Optimisation of in vitro functional assays 219

D.1 Measurements immediately versus 24 h post-thaw . . . . . . . . . . . . 219

D.2 Insulin concentration versus stimulation index . . . . . . . . . . . . 221

D.3 Normalisation of GSIS data . . . . . . . . . . . . . . . . . . . . . 221

$\begin{array}{lll}\text { Appendix E STZ-induced diabetes in NSG mice } & \mathbf{2 2 3}\end{array}$

E.1 Dose of STZ . . . . . . . . . . . . . . . . . . . 223

E.2 Dose of pancreatic islets for transplantation . . . . . . . . . . . 225

E.3 Validation of the portable glucometer . . . . . . . . . . . . . . 227 


\section{List of Figures}

1.1 Islet size distribution and cellular composition in mouse and human pancreas 3

1.2 Regulation of insulin secretion in $\beta$-cells and regulation of blood glucose levels 4

1.3 Human haematopoietic tree and associated markers . . . . . . . . . . . 8

1.4 Schematic of permeation of DMSO into pancreatic islets . . . . . . . . 15

1.5 Schematic of cooling and warming rates in the standard pancreatic islet cryopreservation protocol . . . . . . . . . . . . . . . . 17

1.6 Chemical structure of dimethyl sulphoxide . . . . . . . . . . . . . 21

1.7 Trehalose and its building blocks . . . . . . . . . . . . . 23

1.8 Chemical structure of PLP and its variants . . . . . . . . . . . . . 26

1.9 Structure of antioxidants salidroside and MitoQ . . . . . . . . . . . . . 29

2.1 Method of mouse pancreatic islet isolation . . . . . . . . . . . 37

2.2 Chemical structure and ${ }^{1} \mathrm{H}-\mathrm{NMR}$ spectra of PLP, PP-50, and PP-75 . . . . . 40

2.3 Colony forming units assay. . . . . . . . . . . . . . . . . . . . 49

2.4 A fresh sample of human pancreatic islets stained by dithizone dye . . . . . 50

2.5 Image analysis of islet viability staining . . . . . . . . . . . . 51

2.6 Gating strategy for pancreatic islet flow cytometry . . . . . . . . . . . 53

2.7 Method used for assessing dye diffusion into the centre of pancreatic islets . 54

2.8 ATP/ADP ratio assay reactions . . . . . . . . . . . . . . . . . 55

2.9 Glucose stimulation assay protocol . . . . . . . . . . . . . 57

2.10 Cyclic AMP assay principle . . . . . . . . . . . . . . 60

3.1 Viability assessment considerations for single cells and multicellular aggregates 65

3.2 Comparison of gating strategies for viability assessment of different cryopreservation methods . . . . . . . . . . . . . . 67

3.3 Method of quantification of absolute number of cells and cell subsets surviving cryopreservation . . . . . . . . . . . . . . . . . . 69

3.4 Current imaging method for pancreatic islet viability assessment . . . . . 71 
3.5 Diffusion of nuclear dye into pancreatic islets at room temperature . . . . . 72

3.6 Diffusion of nuclear dye into pancreatic islets with DMSO as a penetration enhancer . . . . . . . . . . . . . . . . . 74

3.7 Effect of hydrostatic pressure on diffusion of nuclear dye into pancreatic islets 76

3.8 Effect of temperature on diffusion of nuclear dye into pancreatic islets . . . 77

3.9 Imaging of pancreatic islet core using confocal, two-photon and light sheet microscopy . . . . . . . . . . . . . . . . . . . . . 79

4.1 Initial efficacy of DMSO, trehalose and PP-50 for cryopreservation of human mononuclear cells from spleen . . . . . . . . . . . . . . . 88

4.2 Initial efficacy of DMSO, trehalose and PP-50 for cryopreservation of human mononuclear cells from bone marrow . . . . . . . . . . . . . . . . 89

4.3 Comparison of three cryopreservation conditions in a 14-day haematopoietic CFU assay . . . . . . . . . . . . . . . . . . . . . . . . 990

4.4 Effects of different cell densities on the outcome of cryopreservation . . . . 93

4.5 Effects of addition of fetal calf serum to DMSO-cryopreserved splenocytes 95

4.6 Effects of addition of fetal calf serum to trehalose-cryopreserved splenocytes 96

4.7 Study design of the thawing protocol refinement . . . . . . . . . . . . . . . 99

4.8 Effect of thawing protocol changes on cryopreservation with DMSO . . . . 100

4.9 Final comparison of thawing protocols for DMSO-cryopreserved samples. . 101

4.10 Effect of thawing protocol changes on cryopreservation with trehalose + PP-50103

4.11 Final comparison of thawing protocols for trehalose + PP-50-cryopreserved samples. . . . . . . . . . . . . . . . . . . . . 104

4.12 Optimisation of length of incubation at $37^{\circ} \mathrm{C}$ before splenocyte cryopreser-

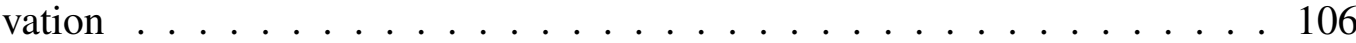

4.13 Optimisation of incubation temperature before splenocyte cryopreservation 108

4.14 Culture of splenocytes with culture medium or $200 \mathrm{mM}$ trehalose . . . . . . 110

4.15 Optimisation of incubation $\mathrm{pH}$ before splenocyte cryopreservation . . . . . 112

4.16 Optimisation of trehalose concentration and incubation time . . . . . . . . 114

4.17 Optimisation of PP-50 and PP-75 polymer concentration . . . . . . . . . 117

4.18 Raman spectroscopy for trehalose uptake measurement and total protein assay 119

5.1 Toxicity of DMSO and trehalose for human and mouse pancreatic islets . . 127

5.2 Design of the DMSO addition protocol study . . . . . . . . . . . . . . . 129

5.3 Comparison of DMSO addition protocols for mouse and human pancreatic islets . . . . . . . . . . . . . . . . . . . . . . . 131 
5.4 Comparison of controlled and uncontrolled freezing for mouse and human pancreatic islets . . . . . . . . . . . . . . . . . . 133

5.5 Design of the islet cryoprotectant removal protocol study . . . . . . . . . 135

5.6 Comparison of thawing protocols for mouse and human pancreatic islets . . 137

5.7 Cryopreservation with trehalose only: concentrations and incubation times . 140

5.8 Supplementation of the DMSO protocol with trehalose . . . . . . . . . . 143

5.9 New insight into the mechanism of survival of cell clusters . . . . . . . . . 145

5.10 Trehalose concentration in the DT6h protocol . . . . . . . . . . . . . 147

5.11 DMSO concentration in the DT6h protocol . . . . . . . . . . . . . . 149

5.12 Effect of salidroside and MitoQ antioxidants addition on post-thaw viability of pancreatic islets . . . . . . . . . . . . . . 151

5.13 In vitro function of pancreatic islets cryopreserved by the optimised protocols 155

5.14 In vivo function of pancreatic islets cryopreserved with DMSO and DT6h protocols . . . . . . . . . . . . . . . . 158

B.1 Gating strategies for enumeration of haematopoietic progenitor cells . . . . 208

B.2 Comparison of cryopreservation protocols efficacy and frequencies of CD34 ${ }^{+}$ cells . . . . . . . . . . . . . . . . . . . . 209

C.1 Toxicity of PP-50 and PP-75 for mouse and human pancreatic islets . . . . 212

C.2 Attachment of fluorescent polymer to pancreatic islet cells . . . . . . . . . 214

C.3 Efficacy of PP-50 and PP-75 in pancreatic islet cryopreservation . . . . . 216

D.1 Optimisation of in vitro functional assays for pancreatic islets and their analysis 220

E.1 Optimisation of STZ dose in the in vivo diabetes model using NSG mice . . 224

E.2 Optimisation of pancreatic islet dose for transplantation under the kidney capsule of diabetic NSG mice . . . . . . . . . . . . . . . . . . . 226 



\section{List of Tables}

1.1 Main cell types in pancreatic islets . . . . . . . . . . . . . . 2

1.2 Overview of most common penetrating and non-penetrating cryoprotectants 11

1.3 Composition of PLP and examples of its amino acid-grafted variants . . . . 25

2.1 Freezing programme for pancreatic islets . . . . . . . . . . . . . . 42

2.2 Cryoprotectant removal protocol for mouse pancreatic islets . . . . . . . 43

2.3 Cryoprotectant removal protocol for human pancreatic islets . . . . . . . . 44

2.4 Antibodies and stains used for the analysis of fresh and thawed mononuclear cells . . . . . . . . . . . . . . . . . . 4 46

2.5 Antibodies and stains used for the fluorescence cell sorting of mononuclear

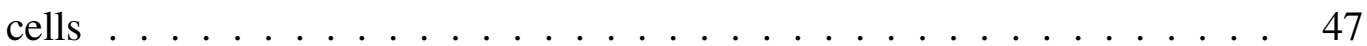

2.6 Antibodies and stains used for the analysis of cells harvested from the colonies of haematopoietic cells grown for the CFU assay . . . . . . . . . . 48

2.7 Composition of Krebs-Ringer Bicarbonate Buffer . . . . . . . . . . . . . . 56

2.8 Components of the PCR mixture . . . . . . . . . . . . . . . . . 58

2.9 PCR programmes for long and short targets . . . . . . . . . . . . 59

4.1 Composition of cryoprotectant solutions . . . . . . . . . . . . . . . 87

4.2 Numbers of deceased human donor samples used for the cell density study . 92

5.1 Protocols for DT6h DMSO concentration experiments . . . . . . . . . . . 148

5.2 Design of antioxidants addition stage experiments . . . . . . . . . . . 153

A.1 Demographics of deceased human organ donors used in experiments . . . 203

A.2 Summary of deceased donor demographics used for each set of experiments. 204

A.3 Clinical and islet isolation information about human islet preparations . . . 205

B.1 Antibody panels used for analysis of cells by flow cytometry . . . . . . . 210 



\title{
List of Abbreviations
}

\author{
Acronyms / Abbreviations \\ 7-AAD 7-Aminoactinomycin D \\ ${ }^{1} \mathrm{H}-\mathrm{NMR} \quad$ Proton nuclear magnetic resonance \\ AF647 Alexa Fluor ${ }^{\circledR} 647$ \\ AFPs Anti-freeze proteins \\ AN Alive nucleated cells; Syto13 ${ }^{+}$7-AAD ${ }^{-}$cell population \\ ANOVA Analysis of variance \\ BM Bone marrow \\ BSA Bovine serum albumin \\ cAMP Cyclic AMP \\ CFU Colony forming unit \\ CFU-GM Granulomonocytic colony forming units \\ DBD Donation after brainstem death \\ DCD Donation after circulatory death \\ DMEM Dulbecco's Modified Eagle Medium \\ DMSO Dimethyl sulphoxide \\ EG Ethylene glycol \\ ESCs Embryonic stem cells
}




$\begin{array}{ll}\text { FCS } & \text { Fetal calf serum } \\ \text { FDA } & \text { Fluorescein diacetate } \\ \text { FSC } & \text { Forward scatter } \\ \text { GIP } & \text { Glucose-dependent insulinotropic polypeptide }\end{array}$

GLP-1 Glucagon-like peptide 1

GMP Good Manufacturing Practice

GSIS Glucose-stimulated insulin secretion

HSC Haematopoietic stem cell

HSD Honest significant differences

IEQ Islet equivalents

iPSC Induced pluripotent stem cell

IVC Inferior vena cava

KRB Krebs-Ringer Bicarbonate Buffer

LT-HSC Long-term haematopoietic stem cell

MitoQ Mitoquinone

mtDNA Mitochondrial DNA

no CPA No cryoprotectant

NSG NOD scid gamma (NOD.Cg-Prkdc ${ }^{\text {scid }} I 12 \mathrm{rg}^{\mathrm{tm} 1 \mathrm{Wjl}} / \mathrm{SzJ}$ ) mouse strain

OCR Oxygen consumption rate

OCT Optimal Cutting Temperature Compound

PCR Polymerase Chain Reaction

PI Propidium iodide

PLP Poly(L-lysine iso-phthalamide)

ROS Reactive Oxygen Species 
SSC Side scatter

ST-HSC Short-term haematopoietic stem cell

STZ Streptozotocin

T Trehalose

TP Trehalose + PP-50

UCB Umbilical cord blood 



\section{Chapter 1}

\section{Introduction}

\subsection{Need for cryopreservation of cells and tissues}

An effective method for long-term preservation of cells and tissues is essential in many fields, particularly in medicine, as a way to overcome the limited time window for the clinical or experimental use of fresh tissues. The shortage of organs for transplantation is currently one of the most pressing challenges in medicine and contributes to significant number of deaths worldwide. Enabling cryopreservation of cells, tissues and organs has a potential to extend the pool of available organs, enable more accurate screening and matching, and remove some of the logistical challenges of transplantation [1].

Freezing of tissues is now routinely performed in fertility treatments, umbilical cord blood stem cell banking or haematopoietic stem cell (HSC) transplantation, but significant advances are needed to enable cryopreservation of other tissues and whole organs. An essential step for the future use of regenerative therapies is development of methods for banking of immunophenotyped embryonic stem cells (ESCs), induced pluripotent stem cells (iPSCs) or other regenerative cellular therapies [2].

Pancreatic islet transplantation is another area in which there is an urgent and current unmet need for an effective cryopreservation strategy. Often it is not possible to obtain sufficient islet cell mass for pancreatic islet transplantation from one donor. Islets from multiple donors are therefore combined to achieve insulin independence in the recipient. However, pancreatic islets can only be cultured for a few days [3], making simultaneous transplantation of fresh islets pooled from several donors an unresolved challenge. This hurdle could potentially be overcome by finding an effective and safe method of islet cryopreservation. 


\subsection{Pancreatic islets}

\subsubsection{Pancreatic islet morphology and function}

The islets of Langerhans, or pancreatic islets, are small distinct cell clusters within the pancreas (constituting about $4.5 \%$ of the adult human pancreas volume [4], found in higher density in the tail region of the pancreas [5]) that are able to secrete endocrine hormones. Pancreatic islets consist of several endocrine cell types, summarised in Table 1.1.

Table 1.1 Main cell types in pancreatic islets

\begin{tabular}{|c|c|c|c|c|}
\hline Cell type & $\begin{array}{l}\text { Hormone } \\
\text { secretion }\end{array}$ & Main function & $\begin{array}{l}\% \text { in human } \\
\text { islets* }\end{array}$ & $\begin{array}{l}\% \text { in mouse } \\
\text { islets* }\end{array}$ \\
\hline$\alpha$-cells & Glucagon & Increasing blood glucose levels & $30-40$ & $15-20$ \\
\hline$\beta$-cells & Insulin & Decreasing blood glucose levels & $50-60$ & $70-80$ \\
\hline$\delta$-cells & Somatostatin & $\begin{array}{l}\text { Inhibition of insulin and } \\
\text { glucagon release }\end{array}$ & $5-15$ & $5-10$ \\
\hline PP-cells & $\begin{array}{l}\text { Pancreatic } \\
\text { polypeptide }\end{array}$ & Reduction in appetite & - & - \\
\hline$\varepsilon$-cells & Ghrelin & $\begin{array}{l}\text { Stimulation of appetite, increased } \\
\text { fat deposition }\end{array}$ & - & - \\
\hline
\end{tabular}

*Percentages of $\alpha, \beta$ and $\delta$-cells in mouse and human islets are derived from Brissova et al. [6].

The organisation of these cell types in islets differs between species: in mouse, most $\beta$-cells populate the central part of the islet and the rest of the cell types predominate on the periphery, while in human islets, the different cell types are more evenly distributed [6]. Although islets significantly vary in size (diameter ranges from $50-250 \mu \mathrm{m}$ ), the diameter of a human islet is on average $109 \mu \mathrm{m}$ [4] and contains between 1,000 - 10,000 cells [7]. In both mouse and human islets, the distribution of islet sizes is similar with relatively high number of small islets and low numbers of large islets [8] (Figure 1.1). In mouse islets, very high proportion of the islets contains $\beta$-cells, regardless of the islet size. Conversely, in human islets, the proportion of $\beta$-cells decreases with the islets size [8] in all head, body and tail of the pancreas [5].

\subsubsection{Regulation of insulin secretion in $\beta$-cells}

Pancreatic $\beta$-cells constantly express a glucose transporter GLUT-2 on their surface, enabling them to continuously sense glucose concentrations in the blood stream. Uptake of glucose by these transporters initiates metabolic and signalling pathways that ultimately lead to release 

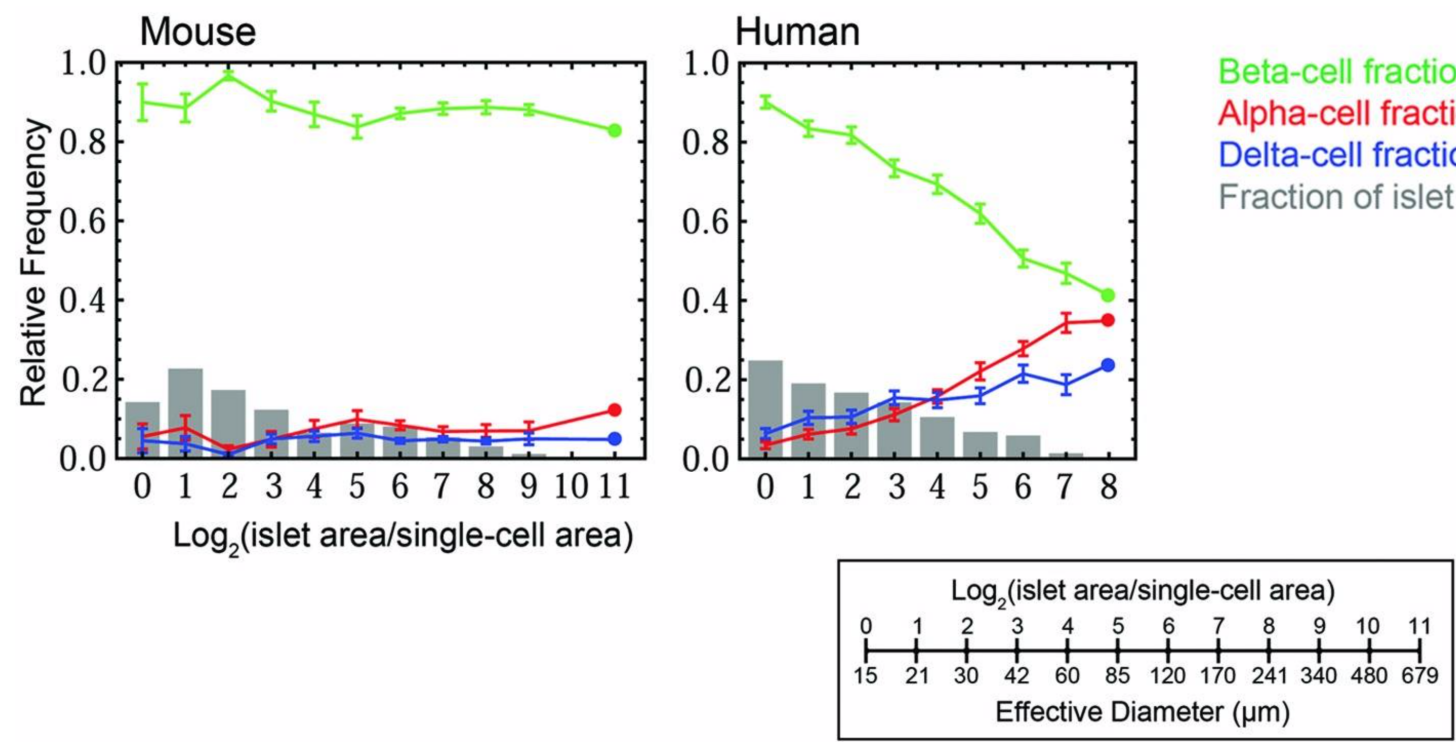

Fig. 1.1 Islet size distribution and cellular composition in mouse and human pancreas. Grey bars represent the frequency of islet size, green, red and blue lines represent the frequency of $\beta, \alpha$ and $\delta$ cells, respectively. Reproduced from Kilimnik et al. [8] with permission from Taylor \& Francis.

of insulin from storage granules, as depicted in Figure 1.2. Insulin release can be further amplified in the presence of incretin hormones such as glucagon-like peptide 1 (GLP-1) or glucose-dependent insulinotropic polypeptide (GIP). These are released by gut cells after food intake and are bound to receptors on the membrane of $\beta$-cells which leads to synthesis of cyclic adenosine monophosphate (cAMP) by adenylyl cyclase and subsequent downstream signalling leading to an increase in insulin degranulation [11].

Insulin release can be inhibited by signalling molecules, including somatostatin, adrenaline and noradrenaline, which target different parts of the secretion pathway (through inhibitory G-proteins or hyperpolarisation of the membrane) [11]. In turn, insulin acts as an inhibitor of glucagon secretion by $\alpha$-cells [12].

\subsubsection{Regulation of blood glucose levels}

As mentioned above, elevated blood glucose levels lead to secretion of insulin by $\beta$-cells. Insulin receptors are present on the membrane of skeletal muscle and adipose cells, as well as hepatocytes. These cell types have membrane glucose transporters (GLUT-4 in muscle and fat cells, GLUT-2 in liver cells) stored in recycling endosomes inside the cells. Unlike $\beta$-cells which constantly express GLUT- 2 transporters on their membrane, these receptors are only exposed on the membrane of muscle cells, hepatocytes and adipocytes after exocytosis of the endosomes is triggered by insulin binding to its receptor. Glucose taken up by these cells 

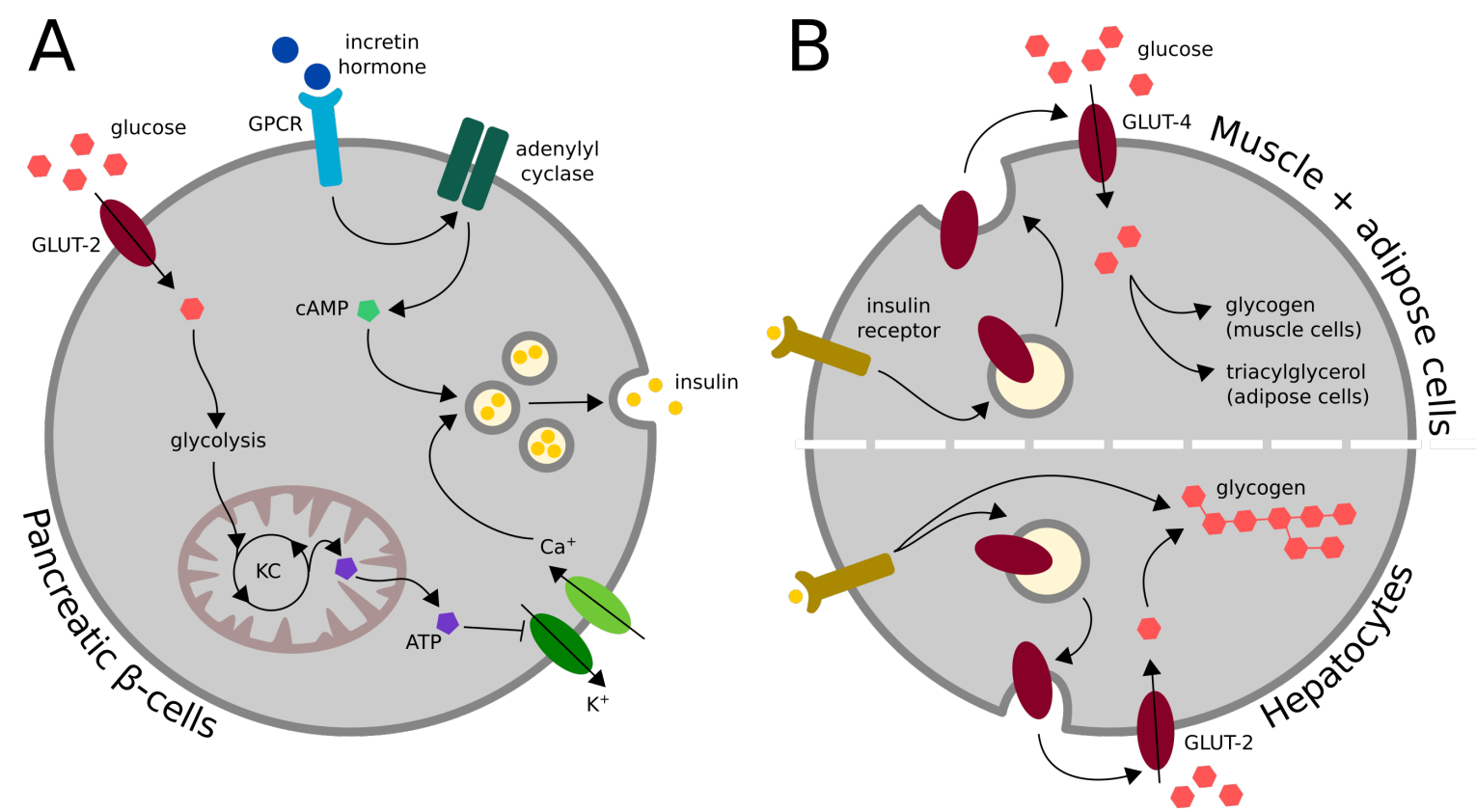

Fig. 1.2 Regulation of insulin secretion in $\beta$-cells and regulation of blood glucose levels. (A) Diagram of glucose sensing and insulin secretion by $\beta$-cells. Glucose is taken up by GLUT- 2 transporter and is catabolised through glycolysis and Krebs cycle, accompanied by synthesis of ATP. ATP-sensitive $\mathrm{K}^{+}$ channels are closed upon the ATP release, causing depolarisation and opening of voltage-dependent $\mathrm{Ca}^{+}$channels. Increased intracellular $\mathrm{Ca}^{+}$concentration then leads to degranulation of the insulin granules and release of insulin into the blood stream. This pathway is necessary for initiation of the insulin secretion in $\beta$-cells. G-protein coupled receptors (GPCRs) on the surface of $\beta$-cells can bind incretin hormones (i.e. GLP-1, GIP) which can in turn stimulate adenylyl cyclase to produce cAMP. This pathway is important for maintaining and amplifying the insulin secretion [9]. (B) In adipose, skeletal muscle and liver cells, GLUT-4 or GLUT-2 transporters would normally be stored in recycling endosomes inside the cells. When insulin is released from $\beta$-cells and binds to an insulin receptor of these cells, signalling cascade induces fusion of these endosomes with cellular membrane, exposing the glucose transporters and enabling glucose uptake. In adipose cells, intracellular glucose would be stored in the form of triglycerols, whereas in muscle cells and hepatocytes it is stored in glycogen molecules. During fasting, these storage molecules can be broken down to glucose and ATP. The detailed signalling pathways and regulation mechanisms differ between cell types. Adapted from Carroll et al. [10] and Rutter et al. [11]. ATP = adenosine triphosphate; cAMP = cyclic adenosine monophosphate; GIP = glucose-dependent insulinotropic polypeptide; GLP-1 = glucagon-like peptide 1; GLUT-2 = Glucose transporter type 2; GLUT-4 = Glucose transporter type 4; GPCR = Gprotein coupled receptor; $\mathrm{KC}=$ Krebs cycle (citric acid cycle). 
can then be stored as glycogen (skeletal muscle and hepatocytes) or triacylglycerol (adipose cells). Thus, concentration of glucose in the blood stream (and in the intracellular space of these cells) is decreased in response to glucose [10].

During fasting, glucagon is secreted by pancreatic $\alpha$-cells and induces breakdown of glycogen in hepatocytes. Similarly, during physical activity, released adrenaline stimulates catabolism of glycogen in skeletal muscles for use as an energy source. This results also in an increased release of glucose into the blood stream [13].

\subsubsection{Pancreatic islets in diabetes treatment}

Diabetes mellitus is a group of medical conditions characterised by increased blood glucose levels, either because of reduced insulin production by $\beta$-cells (type 1 diabetes) or due to acquired resistance to insulin (type 2 diabetes). The treatment for type 2 diabetes initially involves changes in lifestyle, and in more advanced cases the administration of drugs, which can include insulin [14]. Treatment of type 1 diabetes entails tight regulation of blood glucose levels by insulin therapy. Whole pancreas transplantation [15] or pancreatic islet transplantation $[16,17]$ can also be used for management of selected cases and can partly or fully normalise glucose levels.

Whole pancreas transplantation has the disadvantage of being a very invasive procedure with high risk of post-transplant complications. Due to these risks, majority of patients are transplanted with pancreas simultaneously with or subsequently after kidney transplantation as a treatment for end-stage diabetic renal failure. Solitary pancreas transplantation is more unusual as the benefits do not always justify the risk of the transplantation procedure and requirement of immunosuppression [18]. Transplantation of isolated pancreatic islets avoids some of these issues by removing the exocrine portion of the pancreas and by the use of less invasive infusion of the transplanted tissue into the portal vein. However, successful islet transplantation was not clinically attainable for a long time and was only achieved after the optimisation of the isolation procedure [19, 20] and creation of the Edmonton protocol which introduced steroid-free immunosuppression regimen and serial islet transplantations to achieve critical islet mass [18]. According to the Edmonton protocol, 10,000 islet equivalents (IEQ) per kilogram of body weight are administered during pancreatic islet transplantation [21]. Reports suggest that 6,000 to 9,000 islets per kilogram of body weight are sufficient to achieve insulin independence [20]. This means that between 420,000 and 700,000 islets are needed for the transplantation into a $70 \mathrm{~kg}$ patient, however, the typical islet mass gained from one pancreas is 400,000 islets [20]. As a consequence, patients often receive islets from more than one donor on multiple occasions in order to benefit from the 
transplant. In a recent clinical trial, $45.8 \%$ patients received a single islet infusion, $52.1 \%$ received two infusions and $2 \%$ received three infusions [22].

Although pancreatic islet transplantation currently has worse long-term graft survival than whole pancreas transplantation (52\% 5-year pancreas graft survival versus $48 \% 5$-year islet graft survival in the United Kingdom) [23], there is potential for optimisation of the transplantation site, immunosuppressive regimen and dose of islets to further improve outcomes. Indeed, when islet transplantation patients receive an additional islet transplantation from a second or third donor, the 5-year graft survival rises to $60 \%$ [23].

\subsubsection{Cryopreservation in pancreatic islet transplantation}

The need to transplant islets from multiple donors currently leads to subjecting patients to multiple surgeries. In addition, it is preferable for patients to receive their multiple islet transplants in a limited time window after a first pre-conditioning with $\mathrm{T}$ cell-depleting therapy (personal communication with Dr. Shareen Forbes). Cryopreservation of islets from multiple donors and subsequent pooling would enable transplanting the appropriate dose of islets at one time, potentially decreasing the risk of complications coming from the transplantation procedure and increased amount of immunosuppression. It would also allow creation of biobanks and enable effective HLA matching of the islets, which is currently not performed, even though it has been shown to contribute to development of donor-specific antibodies and subsequent rejection of the graft [24].

Early after the introduction of the Edmonton protocol, two diabetic patients were transplanted with a combination of fresh and cryopreserved pancreatic islets and one of these patients remained insulin independent at one year after transplantation. Patients who were transplanted only with fresh islets from a single donor, did not remain insulin independent one year after transplantation due to insufficient dose of transplanted islets [25]. This case report provides a proof of principal in human showing that cryopreserved pancreatic islets can be used to improve transplantation outcome, at least when used in combination with fresh islets.

Protocols using cryopreserved pancreatic islets, however, were never permanently introduced into clinical practice due to low post-thaw survival and the use of fetal calf serum [26]. Better cryopreservation protocols are therefore needed but research on this topic is limited by the low availability of human islets for research and high complexity of cryopreservation protocols due to the fact that islets are multicellular aggregates. For the purposes of this project, human mononuclear cells and mouse pancreatic islets were therefore used for optimisation of the cryopreservation protocols. 


\subsection{Mononuclear cells}

\subsubsection{Sources and use of mononuclear cells}

Mononuclear cells are the fraction of cells from peripheral blood (or other sources) which contain a single spherical nucleus as opposed to red blood cells (no nucleus) or granulocytes (nucleus with multiple lobes). They can be isolated from the whole blood by centrifugation on a density gradient. These cells include primarily immune cells such as lymphocytes and monocytes. Granulocytes and red blood cells are excluded by the gradient separation but some contamination with these cells may remain in the mononuclear fraction.

Mononuclear cells are widely used in immunological and haematological research, as well as in research into blood borne diseases and cancer. There is also increasing interest in development of therapies based on regulatory $\mathrm{T}$ lymphocytes or trans-differentiation of blood cells into other cell types using the iPSC technology. Clinically, they can be used for isolation of haematopoietic progenitors which are later used in HSC transplantation.

Mononuclear cells for therapeutic use are routinely obtained from bone marrow (BM), umbilical cord blood (UCB) or mobilised peripheral blood. Alternatively, they can be found in spleen, but the frequency of the therapeutic haematopoietic progenitors is considerably lower, which then presents a challenge of gathering sufficient number of cells to achieve engraftment after transplantation [27, 28].

\subsubsection{Haematopoietic progenitors and their cryopreservation}

The cell type in the centre of existing clinical therapies derived from mononuclear cells is haematopoietic progenitor cell. Long-term HSCs (LT-HSCs) are thought to be the true multipotent self-renewing population with a generally accepted phenotype of $\mathrm{CD} 34^{+} \mathrm{CD} 38^{-}$ $\mathrm{CD}^{+}{ }^{+} \mathrm{CD}_{45 \mathrm{RA}^{-}}$[29]. Through many developmental stages, including short-term HSCs (ST-HSCs) and lineage-committed progenitors, LT-HSCs give rise to all lineages of the haematopoietic tree (Figure 1.3). For the purposes of transplantation, mononuclear cells are typically enriched for the $\mathrm{CD} 34^{+}$population by magnetic separation [31]. In this thesis, $\mathrm{CD}^{+} 4^{+}$cells will be further referred to as haematopoietic progenitors and these two names will be used interchangably.

HSCs are used for treatment of haematological or other diseases and typically have to be cryopreserved prior to use, except for certain cases of allogenic transplantations that are completed within $72 \mathrm{~h}$ [32]. Avoiding prolonged periods of culture minimises the risk of bacterial or viral contamination and differentiation of the stem cells [33]. The ability to 


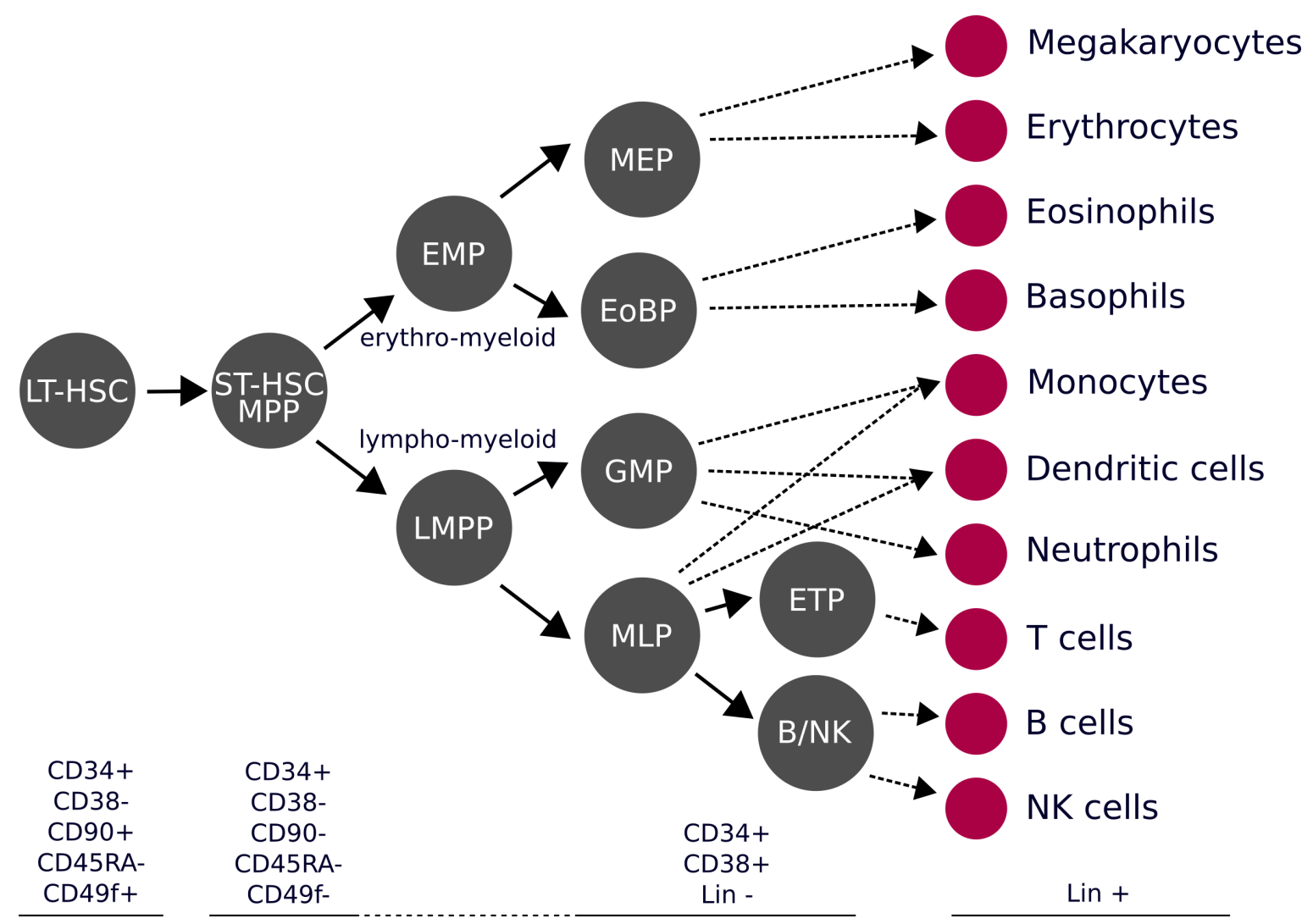

Fig. 1.3 Human haematopoietic tree and associated markers. Adapted from Doulatov et al. [29] with revision from Gorgens et al. [30]. B/NK = B cell and NK cell progenitor; EMP = erythromyeloid progenitor; EoBP = eosinophil and basophil progenitor; ETP = early T-lineage progenitor; GMP = granulocyte and macrophage progenitor; LMPP = lymphoid-primed multipotent progenitor; LT-HSC $=$ long-term HSC; MEP = megakaryocyte and erythrocyte progenitor; MLP = multilymphoid progenitor; MPP = multipotent progenitor; ST-HSC $=$ short term HSC. 
cryopreserve cells allows sufficient time for quality and safety control of the cells or their transport.

Cryopreservation of $\mathrm{CD} 34^{+}$cells is therefore a routine practice and protocols used are summarised in Section 1.6.1. While these protocols can preserve sufficient amount of cells to ensure clinical efficacy of HSC transplantation, improvements on these protocols can have a rapid impact on these existing clinical protocols.

\subsection{Physical aspects of freezing cells}

\subsubsection{Overview of freezing in biological systems}

When mixed water systems cool down, ice crystals begin to form by the process of nucleation. The 'minimal crystal' grows during the freezing process, excluding solutes from its structure which increases the concentration of the solutes in the remaining liquid phase. Formation of ice crystals can be prevented by cooling the system down very rapidly (over $-1000{ }^{\circ} \mathrm{C} / \mathrm{min}$ ), which results in formation of amorphous glass in a process known as vitrification [34].

\subsubsection{Theories and mechanism of cryoinjury}

There is evidence that an optimal freezing rate exists for each cell type. Both exceeding and falling behind of this optimal rate is a source of freezing injury. The two-factor hypothesis proposed by Mazur in 1972 suggests that intracellular ice and changes in solute concentration are the main sources of injury during cryopreservation [35].

If cells are cooled slower than the optimal rate, damage is mostly caused by osmotic stress from the loss of water to extracellular ice formation. As ice forms in the extracellular space, the concentration of the solutes increases, resulting in cellular dehydration to account for the osmotic change. During thawing, the process is reversed and rapid inflow of water into the concentrated cytoplasm represents additional stress factor [35, 36].

Conversely, excessively rapid freezing results in formation of intracellular ice and eventually irreversible damage to the cell membrane or other cellular structures [35, 37]. This risk is present not only during the freezing but also during thawing when recrystallisation is accompanied by growth of crystals, especially when the warming is performed slowly $[35,36]$.

\subsubsection{Mechanisms of cryoprotection}

Many organisms living in cold areas are adapted for survival in sub-zero temperatures. Coldtolerant species utilise a variety of mechanisms to mitigate the detrimental effects of freezing. 
These include production of glucose, disaccharides, polyols, ice nucleators, and specialised antifreeze proteins or glycolipids [38].

Inspired by the nature, the protective effects of the first cryoprotectants were discovered in 1940s and 1950s, enabling cryopreservation of cells. A cryoprotectant is a substance which, when added to the freezing medium, is capable of reducing the freezing injury to the cryopreserved cells. The underlying mechanism of cryoprotection involves lowering the solute concentration by increasing the intracellular osmotic pressure. This lowers the freezing point of the system, thus reducing the incidence of ice crystal formation $[34,39]$.

An alternative method of preventing damage by ice crystal formation is vitrification of the cells, which involves formation of a non-crystalline amorphous solid state. However, achieving vitrification in many biological systems is challenging because cryopreservation is performed in volumes too large to achieve homogenous freezing. Vitrification is not possible without elimination of water-water interactions which is usually achieved by increasing the concentration of the cryoprotectant, often to the point where it exerts toxic effects on the cells [40].

\subsection{Cryoprotectants}

\subsubsection{The ideal cryoprotectant}

An effective cryoprotectant should be able to protect cells from the damage caused by ice crystal formation, while reducing the osmotic stress, and subsequently allow cells to return to their normal physiological state. Cell membrane integrity, cell volume, and other morphological qualities of the cells should be preserved as well. It must also maintain the genomic and epigenetic characteristics of the cells, their expression profile, and thus their physiological function after thawing. It must not induce differentiation or de-differentiation of cells. Furthermore, to be applicable in clinical and research practice, attention must be paid to the cost of the method, the time of the pre-freeze and post-thaw processing, transport requirements and storage space requirements. A cryoprotectant used in clinical practice must meet additional requirements related to patient safety.

The ideal cryopreservation protocol will most likely not be identical for all cell types because of differences in cell size, water content, intracellular structures, membrane composition, and cell density. The cryopreservation protocols must therefore be tailored to various cell types [34]. Special attention also needs to be paid to cryopreservation of cell clusters, since the protocol must allow the cryoprotectant to penetrate the cells located centrally within the cluster, making diffusion kinetics a central parameter. 


\subsubsection{Overview of cryoprotectants}

The first study utilising glycerol as a cryoprotectant showed that addition of a protective agent can significantly increase the survival rate of spermatozoa [41]. Further research showed other neutral solutes with low molecular weight, such as ethylene glycol and diethylene glycol, can have similar effects as glycerol. Sugars (glucose and xylose) and sugar alcohols (erythritol) can also provide some, albeit reduced, level of protection, mostly because the cell membrane is only partially permeable to them [39]. Subsequently, dimethyl sulphoxide (DMSO) was identified as a potentially ideal cryoprotectant, preferred to glycerol because of the higher permeability of cell membrane to DMSO [42]. Glycerol and DMSO still remain the most commonly used cryoprotectants in clinical practice and research.

Currently available cryoprotectants can be classified into two groups based on their ability to cross the plasma membrane: penetrating or non-penetrating. Penetrating cryoprotectants, such as DMSO, glycerol or other alcohols, can easily enter the cell and replace a fraction of water molecules in the intracellular space, preventing dehydration after outflow of water during the cooling process. Conversely, non-penetrating cryoprotectants such as various mono-, di- and polysaccharides, in most cases cannot permeate through the membrane and thus only act extracellularly [43]. The mechanism of extracellular cryoprotection lies in modification of the membrane permeability which helps to maintain the cell volume in a tolerable range [44]. Examples of penetrating and non-penetrating cryoprotective substances are shown in Table 1.2.

Table 1.2 Overview of most common penetrating and non-penetrating cryoprotectants

Penetrating cryoprotectants

$\begin{array}{ll}\text { Sulphoxides } & \text { Dimethyl sulphoxide } \\ \text { Amides + imides } & \text { Formamide, acetamide, propionamide etc. } \\ \text { Alcohols } & \begin{array}{l}\text { Glycerol, methanol, ethylene glycol, propylene glycol, erythritol, } \\ \text { mannitol, sorbitol }\end{array}\end{array}$

Non-penetrating cryoprotectants

Monosaccharides Glucose, galactose, arabinose, mannose, fructose etc.

Disaccharides Sucrose, trehalose, lactose, maltose etc.

Polysaccharides Raffinose, dextran, hydroxyethyl starch

Macromolecules Ficoll, polyethylene glycol, polyvinylpyrrolidone, polyvinyl alcohol, hyaluronan

Adapted from Swain et al. [43] with revisions from Meryman [44] and Stolzing et. al [45] 


\subsubsection{Mechanisms of cryoprotectant toxicity}

While cryoprotectants can prevent irreversible cell damage during freezing, they may themselves have toxic effects. This toxicity limits the concentration of the cryoprotectant that can be used for cryopreservation and therefore its protective potency [46]. To be considered toxic, it must be clearly demonstrated that the cryoprotectant causes significant and irreversible changes to cells detrimental to survival and function in relevant concentration ranges, exposure times and temperatures $[43,47]$. Mechanism of toxicity of cryopreservation solutions has not been fully clarified but variety of harmful effects have been observed.

Toxic effects of cryoprotectants can be categorised as non-specific (relevant to all cryoprotectant) or specific (related to individual cryoprotectants or group of cryoprotectants). Non-specific effects include cell dehydration, changes in membrane permeability, or external environment polarity [46]. Specific toxic effects include chemical modifications of cellular structures or enzymes, changes in cytoskeleton, changes in transcription profile, and many others $[46,47]$. Toxic effects associated with DMSO are discussed in detail in Chapter 1.8.2.

Reduction of cryoprotectant toxicity can be achieved by step-wise cryoprotectant addition and removal which reduces the osmotic stress to the cells [43]. Use of lower concentrations and shorter exposure times reduce the toxicity but often also the efficacy. Toxic effects of individual cryoprotectants can also be diminished by combining several cryoprotectants in lower and less toxic concentrations [43] or by toxicity neutralisation by other substances [48].

\subsection{Current cryopreservation methods}

\subsubsection{HSC cryopreservation protocols}

\section{Choice of cryoprotectant}

Current protocols for cryopreservation of haematopoietic progenitors in clinical and research practice differ significantly between institutions. The predominant cryoprotectant used is DMSO at $10 \%$ concentration, but lower concentrations of DMSO are also used [32, 49] with varying degrees of success $[50,51]$. DMSO-containing cryopreservation solutions are usually supplemented by serum, as this has been shown to have a beneficial effect on cell recovery [52]. Other additives such as hydroxyethyl starch [53] or disaccharides [54, 55] are sometimes used. Protocols that do not utilise DMSO are yet to be clinically validated. 


\section{Freezing rate}

In clinical practice, controlled-rate freezers are recommended for freezing of haematopoietic progenitors [56]. The programmed freezing rate should reflect the specific needs of the cell type and the cryoprotectant used. It must also compensate for the heat released during transition from liquid to solid phase by increasing the freezing rate around the eutectic point (temperature where all the components of a mixture freeze) [57]. Nevertheless, uncontrolled freezing may be preferred to use of a controlled-rate freezer due to its lower cost. Moreover, uncontrolled freezing has been shown to have comparable efficacy to controlled-rate freezing for these cells $[53,58]$ with the exception of granulomonocytic colony forming units (CFUGM) growth efficiency where uncontrolled freezing results in slightly worse outcomes [59]. The ideal freezing rate for human haematopoietic progenitors cryopreserved in $10 \%$ DMSO was shown to be between 1 and $3{ }^{\circ} \mathrm{C} / \mathrm{min}[60]$.

\section{Cryopreservation temperature}

Long-term storage of samples in mechanical freezers with temperatures of approximately $-80{ }^{\circ} \mathrm{C}$ is not ideal since the temperature is not low enough to completely stop the biochemical activity of some enzymes, which may have implications for cell viability [61]. Conversely, storage in liquid phase of nitrogen $\left(-196^{\circ} \mathrm{C}\right)$ carries the risk of contamination [62]. This can be partly avoided by keeping the samples in the nitrogen vapour phase or in more efficient mechanical freezers (temperatures as low as $-150{ }^{\circ} \mathrm{C}$ ).

\section{Cell density}

Another variable in cryopreservation protocols is cell concentration. Although cell concentrations below $2 \times 10^{7}$ cells/mL were initially preferred due to toxicity concerns [32], later studies validated the use of higher cell densities (up to $5 \times 10^{8}$ ) for successful HSC cryopreservation $[63,64]$. Cryopreservation in higher cell densities implies lower final volume, reducing the cost of storage and risk to the patient resulting from the amount of cryoprotectant infused into the body as part of the clinical therapy. No significant differences were found between storage of cells in vials and bags [65].

\section{Thawing and post-thaw processing}

Standard thawing protocols for haematopoietic progenitors used by most institutions includes rapid thawing in $37^{\circ} \mathrm{C}$ water bath although slower thawing at $20^{\circ} \mathrm{C}$ does not significantly decrease the post-thaw viability of cryopreserved haematopoietic progenitors [66]. The 
optimal rate of thawing has been shown to be dependent on the rate of freezing. Slow warming is more damaging for rapidly frozen cells than slowly frozen cells but generally, rapid thawing results in enhanced recovery regardless of the freezing rate [67]. Concerns about contamination caused by thawing in water bath have been addressed by development of a dry heat device [68].

Subsequent washing of the cells is recommended to ensure the removal of the cryoprotectant. It usually includes slow dilution of the cryoprotectant to reduce the osmotic shock and removal of the supernatant after centrifugation [69]. Although this removes most of the cryoprotectant and improves the viability and clonogenic activity of the cells, it also results in significant cell losses (approximately $30-40 \%[69,70]$ ). For clinical purposes, automated methods of post-thaw washing have been developed [70].

\subsubsection{Pancreatic islet cryopreservation protocols}

Compared to single cell cryopreservation, freezing and thawing of cell clusters such as pancreatic islets presents additional challenges. The size of the islets makes it necessary to incubate them longer with the cryoprotectant to allow for cryoprotectant diffusion throughout the cluster and its equilibration with the water inside cells. During the incubation and cryopreservation, the different cell layers will be subjected to different cryoprotectant and intracellular water concentrations and non-uniform temperature changes, which can lead to increased ice crystal formation in some regions of the tissue [71]. Moreover, the size variability between islets (shown earlier in Figure 1.1) makes it nearly impossible to design an ideal incubation and freezing protocol if islets are cryopreserved in a heterogeneous batch. Important parameters of the pancreatic islet cryopreservation are summarised below.

\section{Addition of cryoprotectants}

Most adult pancreatic islet cryopreservation protocols use DMSO as a cryoprotectant. If islets are incubated in a single concentration of DMSO, its penetration into the centre of the cluster is not rapid enough to allow for equilibration and avoid its high time-dependent toxicity at the same time (Figure 1.4). Multi-step protocols were therefore designed to first allow equilibration of low and less toxic concentrations of DMSO, followed by short incubation in the final DMSO concentration. Step-wise addition protocols also minimise the osmotic stress from addition of high concentrations of solutes [73].

The current standard protocol designed by Rajotte suggests incubation in 0.67 M DMSO for $5 \mathrm{~min}$ at $22{ }^{\circ} \mathrm{C}$, followed by incubation in $1 \mathrm{M} \mathrm{DMSO}$ for $25 \mathrm{~min}$ at $22{ }^{\circ} \mathrm{C}$ before final incubation in $2 \mathrm{M} \mathrm{DMSO}$ for $15 \mathrm{~min}$ at $0{ }^{\circ} \mathrm{C}$ [74]. 


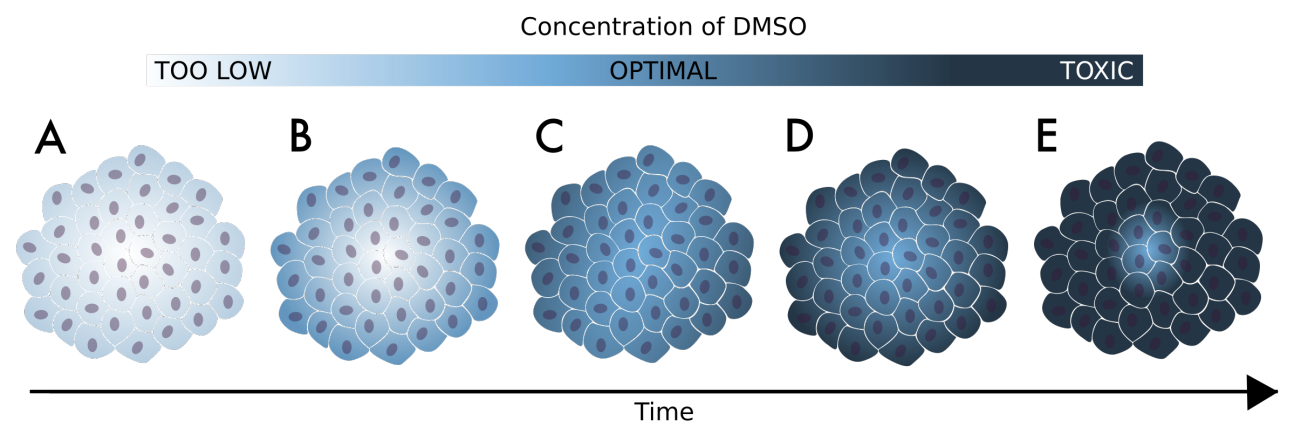

Fig. 1.4 Schematic of permeation of DMSO into pancreatic islets. In pancreatic islets immersed in DMSO solution (single concentration), DMSO diffuses into the core in a time-dependent manner. (A) and (B) Short incubation times do not provide sufficient concentration of DMSO to provide cryopreservation effect in the whole islet volume. (C) (D) and (E) Later, the prolonged exposure of the peripheral cells to DMSO starts exhibiting its toxic effect. Achieving homogenous concentration of DMSO in the whole islet volume is not possible using simple incubation in single concentration of DMSO. The time scale on this schematic depends on the size of a particular islet; it has been shown that an islet of $150 \mu \mathrm{m}$ diameter can equilibrate with $1.5 \mathrm{~mol} / \mathrm{kg}$ DMSO in $\sim 10 \mathrm{~min}$ at room temperature [72]

Diffusion rates and ideal incubation steps are highly likely to be different for other cryoprotectants (e.g ethylene glycol, glycerol or non-penetrating cryoprotectants), although many have not yet been experimentally determined. Mazur and Rajotte have provided early evidence of permeation rates for DMSO and glycerol in fetal rat pancreas: full equilibration with $2 \mathrm{M}$ DMSO was achieved in only $10-15$ min at $0{ }^{\circ} \mathrm{C}$ while it took $10-30$ times longer to achieve equilibration for glycerol [75]. Evidence from other publications suggests that the standard 45 min incubation protocol with DMSO should allow for full equilibration of DMSO in islets [76]. Benson et al. have shown that a $150 \mu \mathrm{m}$ diameter islet can equilibrate with $1.5 \mathrm{~mol} / \mathrm{kg}$ in $\sim 10 \mathrm{~min}$ at room temperature [72].

\section{Cryoprotectant mixtures}

The current standard method uses DMSO at $2 \mathrm{M}$ final concentration. Studies examining the ideal final concentration of DMSO are limited. Lakey et al. have shown in human islets that decreasing the concentration to $1.5 \mathrm{M}$ does not negatively impact the post-thaw islet recovery and insulin secretion [77]. Conversely, another group has performed similar experiments with rat islets but found that decreasing DMSO concentration from $14.4 \%$ (equivalent to $2 \mathrm{M}$ ) to $10 \%$ (equivalent to $1.4 \mathrm{M}$ ), as well as increasing it above $14.4 \%$ resulted in lower viability [78]. 
Taylor et al. compared DMSO and glycerol for cryopreservation of adult rat pancreatic islets and found that DMSO was superior to glycerol in preserving post-thaw insulin secretion patterns and ability to reverse diabetes in rats [79].

Ethylene glycol (EG) was successfully shown in a rat model to be a less toxic alternative to DMSO [80], with EG achieving a higher recovery count and DNA content per islet, higher glucose-stimulated insulin secretion and ability to reach insulin independence in diabetic mice [81]. However, these results have not been replicated using human islets [82].

Beattie et al. tested addition of $11.1 \mathrm{mM}$ glucose and $300 \mathrm{mM}$ trehalose to a DMSObased cryopreservation medium. They cryopreserved adult and fetal-like pancreatic islets and observed a significant increase in recovery when trehalose was added together with DMSO for both tissue types compared to glucose addition. However, they did not directly compared the protocol to the original one proposed by Rajotte et al. [83].

\section{Freezing and thawing rate}

Slower freezing rates are considered to be beneficial in slow-cooling (as opposed to vitrification) since they allow sufficient time for water to escape the cells which then prevents formation of intracellular ice [35]. Rajotte noticed that freezing rate for cell clusters tends to be slower than for single cells and suggested the freezing rate of $-0.25{ }^{\circ} \mathrm{C} / \mathrm{min}$ for pancreatic islets. Rajotte's full freezing protocol includes a seeding stage at $-7.5^{\circ} \mathrm{C}$ for $10-15 \mathrm{~min}$ to allow for nucleation and release of latent heat of fusion, slow-cooling to $-40{ }^{\circ} \mathrm{C}$ and plunging to liquid nitrogen [73] (Figure 1.5). Other groups use cooling rates of -0.25 or $-0.3{ }^{\circ} \mathrm{C} / \min [79]$.

Foreman et al. compared different cooling rates for islets partially or fully-equilibrated with DMSO and found that the cooling rates from -0.3 to $-1000{ }^{\circ} \mathrm{C} / \mathrm{min}$ were equivalent as long as the islets fully equilibrated with the cryoprotectant [76].

Regarding thawing rate, Rajotte observed that if islets were slow-cooled to $-40{ }^{\circ} \mathrm{C}$ they required rapid thawing. Conversely, slow thawing was ideal for samples slow-cooled to $-75{ }^{\circ} \mathrm{C}$ [73]. Current protocol includes thawing of cryovials in $37{ }^{\circ} \mathrm{C}$ water bath until the last small crystal is visible which usually achieves thawing rates between 150 and $200{ }^{\circ} \mathrm{C} / \mathrm{min}$ [84]. It has been shown that rapidly thawed islets recovered better post-thaw compared to islets thawed slowly at room temperature [85].

\section{Cryoprotectant removal}

For human pancreatic islets, the recommended procedure for DMSO removal is dilution by $0.75 \mathrm{M}$ solution of sucrose followed by equilibration for $30 \mathrm{~min}$, and step-wise addition of 


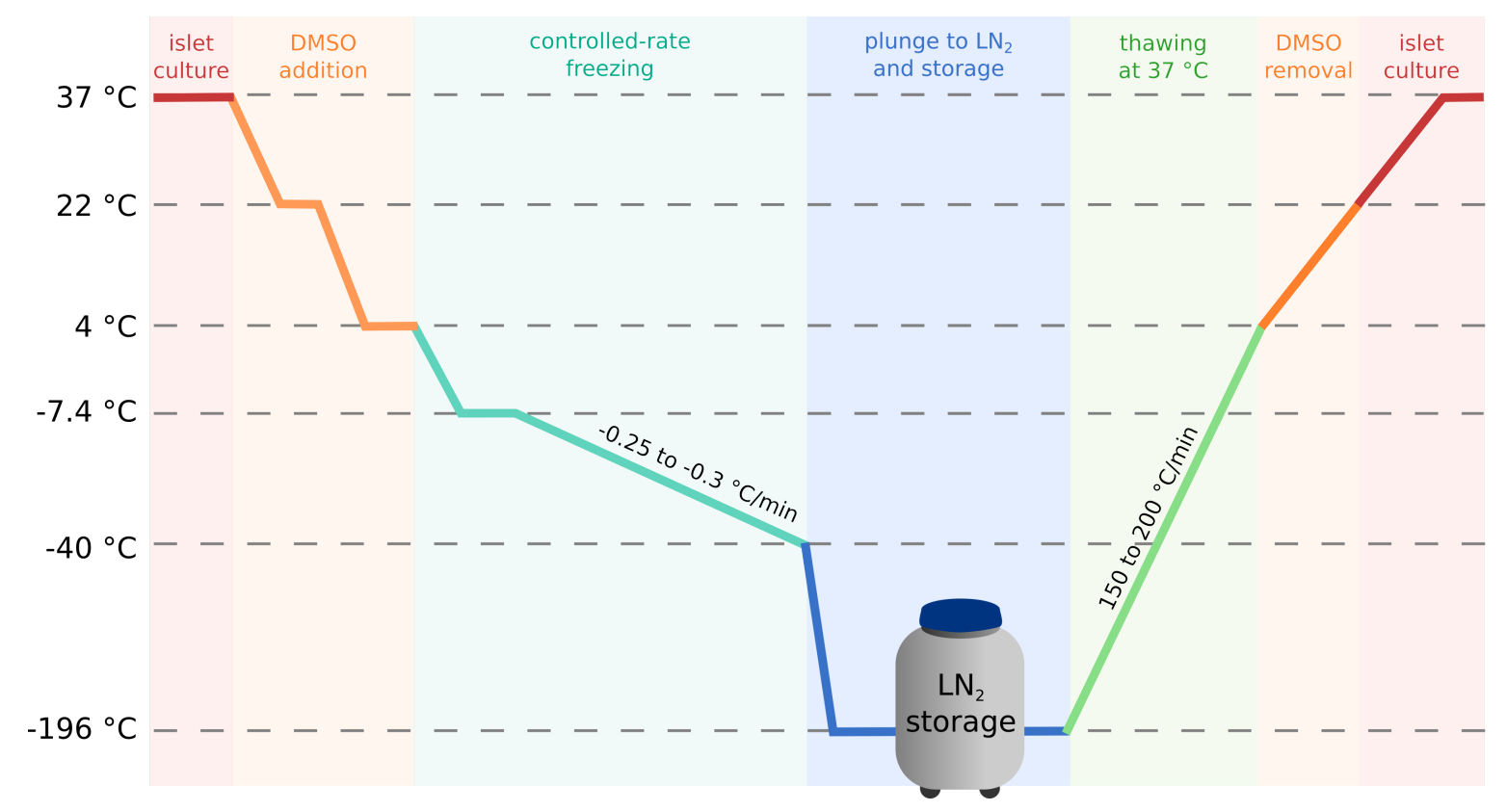

Fig. 1.5 Schematic of cooling and warming rates in the standard pancreatic islet cryopreservation protocol. Pancreatic islets are brought from the $37^{\circ} \mathrm{C}$ culture to room temperature, at which point the DMSO is added step-wise. Once the final DMSO concentration has been reached, islets are transferred to $4{ }^{\circ} \mathrm{C}$ and further incubated for $15 \mathrm{~min}$, before controlled-rate freezing commences. Samples are cooled down to $-7.4{ }^{\circ} \mathrm{C} / \mathrm{min}$ and after allowing for nucleation and release of the latent heat of fusion, cooling rate of -0.25 or $-0.3{ }^{\circ} \mathrm{C} / \mathrm{min}$ is used to bring the samples down to $-40{ }^{\circ} \mathrm{C}$. At the end of this freezing programme, samples are plunged to liquid nitrogen and stored in a dewar until thawing. Thawing is performed by immersing vials in $37{ }^{\circ} \mathrm{C}$ water bath until the last crystal is visible, bringing the islets into physiological medium by step-wise dilution of DMSO, upon which islets can be cultured at $37^{\circ} \mathrm{C}$ again. Figure adapted from Rajotte [73]. $\mathrm{LN}_{2}=$ liquid nitrogen. 
thawing medium every $5 \mathrm{~min}$. A $24 \mathrm{~h}$ post-thaw culture at $37{ }^{\circ} \mathrm{C}$ has also been suggested to allow a recovery period for the islets prior to in vitro or in vivo testing $[73,86]$.

Different methods of thawing have been suggested for islets isolated from dogs. The method of Lakey et al. omits the sucrose dilution and simply uses a step-wise dilution of DMSO over $24 \mathrm{~min}$. They showed that this method results in equivalent islet survival when cryopreservation is done in glass tubes and superior to the sucrose method when freezing is done in freezer bags [87].

\subsection{Current methods of cryosurvival assessment}

\subsubsection{Assessment of mononuclear cell cryosurvival}

\section{Methods used in clinical transplantation and cryobiology}

The most common methods used for assessment of viability of mononuclear cells (and other single cell suspensions) are trypan blue staining and flow cytometry. Trypan blue offers a rapid non-specific viability assessment of all cells in the mixed population of mononuclear cells and is performed under a light microscope using haemocytometer. Flow cytometry offers the option of labelling and distinguishing different cell types, a useful feature if a particular sub-population is of interest. The disadvantages of flow cytometry are longer time, higher cost and specialised equipment requirements.

Adaptation of these methods for the purposes of cryobiology has been noted in some studies [88]. Yang et al. noticed that cryosurvival is reported inconsistently in different publications. Reporting only the viability of the post-thaw sample overestimates the actual efficacy of cryopreservation since it does not account for the loss of cells during the freezing process. It does, however, give a useful evaluation of the sample quality. More accurately, percentage survival should be reported which relates the number of viable cells after thawing to the number of viable cells pre-freeze [89]. However, this recommended practice of reporting viability along with absolute counts has not been rigorously followed and no standard method for cryobiological assessment of mononuclear cells exists to date.

\section{Survival of haematopoietic progenitors}

Because of the clinical use of haematopoietic progenitors for HSC transplantation, assessment of their survival has been a well explored area. The focus of the community has been the standardisation of haematopoietic progenitor markers, flow cytometry gating strategies and cell enumeration method between clinical centres. The widely standardised ISHAGE 
guidelines for enumeration of $\mathrm{CD} 34^{+}$cells are routinely used in clinical practice and use a gating strategy based on light scatter properties of cells and fluorescence from anti-CD45 antibody conjugated to FITC fluorochrome, anti-CD34 antibody conjugated to PE fluorochrome and 7-AAD dead cell dye [90].

This method, however, only uses very crude determination of progenitor capacity by using the CD34 as a marker which labels all early and late progenitors. To identify true stem cells, additional markers should be used in flow cytometry measurement (see Figure 1.3), or the ability to differentiate into multiple lineages should be evaluated by functional assays. The widely used in vitro functional assay for haematopoietic progenitors is the colony forming units (CFU) assay. It can determine the proliferation and differentiation potential of progenitors, mainly towards the myeloid and erythroid lineages. An in vivo assay, consisting of depletion of bone marrow cells by irradiation of mice followed by transplantation of haematopoietic progenitors can also be used for identification of LT-HSCs [91].

\subsubsection{Assessment of pancreatic islet cryosurvival}

Many of the methods used for assessment of islet survival are very specific due to the fact that these are 3D cellular clusters which vary significantly in size and shape. A variety of methods have been developed for assessment of islets before transplantation but most have important limitation in predicting clinical outcomes. In cryobiology research, the protocols used for survival assessment mostly follow the methods used in clinical transplantation described below.

\section{Islet viability}

Staining islets with a combination of membrane integrity stains and dye based on enzymatic activity of cells is the most common method for islet viability measurement. These dyes include propidium iodide (PI), ethidium bromide or ethidium homodimer-1 for staining of dead cells and fluorescein diacetate (FDA), acridine orange or Syto ${ }^{\circledR}$ and Sybr® dyes for staining of live cells [92]. Despite its wide-spread use, especially of the FDA/PI combibation, it has been shown that viability determined by this method does not correlate with clinical outcomes [93-95].

There is little current insight into the reasons underlying this discrepancy. Potential reasons include inability of these dyes to detect apoptotic cells [95] and a biased method of analysis. Currently, islets stained with viability dye are commonly manually categorised based on the perceived viability [94]. This method of image analysis is highly subjective 
and therefore automated image analysis programmes should ideally be employed to avoid variability between assessors.

Assays using dissociated islets are in some aspects less biased since precise methods such as flow cytometry can be used. However, during the dissociation process, islets may be subjected to additional damage caused by enzymatic digestion or mechanical stress (based on the dissociation method used) and cell losses are high. After dissociation, cell death rate also potentially increases because of the loss of inter-cellular contact and communication which is required by many tissues, including islets [96].

Basic viability staining of both intact and dissociated islets, nevertheless, remains a method widely used in the clinical practice due to its simplicity and because it is one of the few assays that can be performed prospectively - i.e. before the transplantation.

\section{Islet size}

Size of islets is an important parameter in clinical transplantation and currently determines the dose of islets given to a patient. However, it is challenging to measure, especially if exocrine tissue is also present in the sample. A classic method of size and purity measurement involves staining with dithizone (a dye specific for insulin producing cells due to their high content of zinc) and manual categorisation of islet size observed under a light microscope using a calibrated grid. The results is then expressed as islet equivalents (IEQ, one IEQ is equivalent to an islet of $150 \mu \mathrm{m}$ diameter) [97]. New image analysis methods and use of novel technologies (such as large particle flow cytometry or nuclei counting) were suggested but have not been introduced into clinical practice [95].

\section{Islet function in vitro}

Glucose-stimulated insulin secretion (GSIS) is a classical assay used for evaluation of islets' ability to respond to varying concentrations of glucose. This assay can be done either in a static culture or as a perifusion measurement where islets are placed in a solution flow of changing glucose concentrations followed by continuous sampling of the secretions. The results obtained from the static assays have been useful for predictions of transplantation outcome in some [94] but not other studies [98, 99]. Variability in the results of this functional assay may be caused by insulin degranulation by damaged cells, variable assay conditions or the absence of necessary signal molecules [95].

Two methods focusing on function of mitochondria have been recently suggested to replace current viability and functional measurements. The advantage of mitochondrial assays is that they are able to detect dying cells at the apoptosis stage, unlike standard viability 
measurements. Papas et al. suggested measuring oxygen consumption rates (OCR) of intact islets. They have shown that OCR and OCR to DNA ratio correlate with transplantation outcomes in diabetic mice [100]. Measuring the ATP/ADP ratios has also shown promise in accurate prediction of in vivo results [101].

\section{Islet function in vivo}

Demonstrating reversal of diabetes in vivo is the ultimate measure of pancreatic islet function. Transplantation of pancreatic islets under the kidney capsule of immunodeficient mice has been shown to correlate with the outcome of clinical transplantation and is therefore regarded as the most reliable assay for assessment of islet quality. The downsides of this approach include long duration, technical difficulty and cost of the assay as well as the necessity to time the diabetes induction with availability of islets [95].

\subsection{DMSO}

\subsubsection{DMSO attributes}

Dimethyl sulphoxide (chemical formula $\left(\mathrm{CH}_{3}\right)_{2} \mathrm{SO}$ ) is a compound composed of a polar sulphoxide moiety connected to two non-polar methyl moieties (Figure 1.6). This structure gives DMSO high affinity to water; one molecule of DMSO is thought to bind two water molecules by hydrogen bonds. This interaction decreases the freezing point of the solution [102].<smiles>CS(C)=O</smiles>

Fig. 1.6 Chemical structure of dimethyl sulphoxide. Polar part of the molecule consists of sulphoxide, attached to the two methyl groups with non-polar character.

The cell membrane is highly permeable to DMSO. This characteristic was initially thought to be the predominant mechanism of its cryoprotective properties [42], allowing it to equilibrate on both sides of the membrane easily and thus prevent both ice crystal formation and dehydration [54]. Subsequently, its high cryoprotective efficiency was also attributed to its colligative properties (dependent on the concentration of the substance, not on its specific chemical properties) [103], but the possibility of its direct interaction with other molecules and cellular structures was also explored. Anchordoguy and colleagues showed that the 
oxygen atom in DMSO interacts with positively charged phospholipid heads [104]. Another mechanism of protection includes the preferential exclusion of DMSO from the vicinity of proteins at low temperatures, which keeps proteins surrounded by water molecules and prevents their denaturation [105].

\subsubsection{DMSO toxicity}

Despite of its cryoprotective properties, DMSO can exert toxic effects that are depending on its concentration, exposure time, and temperature [33]. Although cells can tolerate even high concentrations $(25 \%)$ of DMSO at $2{ }^{\circ} \mathrm{C}$ for up to one hour, the same exposure of haematopoietic progenitors at $20^{\circ} \mathrm{C}$ results in a significant decrease in viability and ability to form haematopoietic colonies [106]. DMSO can exert a range of adverse cellular effects, such as changing the dynamics of cytoskeleton [107], altering the activity of enzymes involved in glycolysis and other biochemical processes [108] or causing protein denaturation [109]. In regenerative medicine, there is a concern about the effect of DMSO on protein expression by embryonic stem cells [110] or epigenetic profile of embryoid bodies [111].

Toxicity of DMSO does not only has an impact at the cellular level, but cases of adverse effects in transplant patients have also been observed after infusion of DMSOcryopreserved cells. In many cases, DMSO toxicity manifests as nausea, vomiting, and abdominal cramps [49]. However, more serious effects can also be associated with administration of DMSO, including cardiovascular, neurological, gastrointestinal and renal complications or allergies (reviewed by Shu et al. [112] and Cox et al. [113]). Transplant centres are being encouraged to use lower concentrations of DMSO or to remove it before administration to patients but this has not become standard practice [49].

\subsubsection{Alternatives to DMSO}

Attempts have been made to supplement or substitute DMSO with naturally occurring protective substances such as saccharides. The disaccharides sucrose and trehalose are the most promising candidates. They can both form 'glass' and stabilise proteins and membranes, although trehalose is a more effective cryoprotectant than sucrose [114]. One possible explanation is trehalose's larger hydration radius which enables it to better protect proteins by preferential exclusion of the sugar from the proteins, in a manner similar to DMSO [115]. It is also more effective than sucrose in replacing water molecules in the hydration layer of the phospholipid membrane and thus provide greater protection against damage from dehydration [116]. Moreover, trehalose is less prone to hydrolysis than sucrose in suboptimal conditions such as low $\mathrm{pH}[117]$. 


\subsection{Trehalose}

\subsubsection{Trehalose attributes}

The disaccharide trehalose is composed of two D-glucose units linked by an $\alpha, \alpha-1,1-$ glycosidic bond (Figure 1.7). This link gives the disaccharide a non-reducing character and prevents its degradation in acidic conditions or by the activity of $\alpha$-glucosidase. Trehalose is naturally produced by many lower organisms and metabolised by trehalase enzyme [118]. Although unable to synthesise trehalose, numerous higher organisms, including humans, express trehalase in the small intestine and other organs and are therefore able to digest the disaccharide $[118,119]$. Trehalose has been shown to cause little or no toxicity in humans [118], with the exception of very rare cases of trehalose intolerance [120].

Several hypotheses have been proposed to explain the cryoprotective effects of trehalose. The water-replacement hypothesis suggests that trehalose replaces water molecules in binding polar groups of molecules and other cellular structures (most importantly the cell membrane) and therefore aids in preserving their conformation during freezing [121]. Water entrapment or preferential hydration hypothesis suggests that trehalose does not bind directly to the surface of proteins but instead surrounds thin layer of water molecules around the protein, keeping it hydrated $[115,122]$. The vitrification hypothesis suggests that trehalose allows the formation of amorphous glass which minimises the mechanical damage caused by crystal formation [123]. Additional evidence suggests that trehalose can promote stability of proteins by lowering the oxidative stress $[124,125]$ which could be advantageous for highly sensitive cells such as haematopoietic progenitors [126] or pancreatic islets [127]. These mechanisms may all contribute to the protective effects of trehalose [118].

A
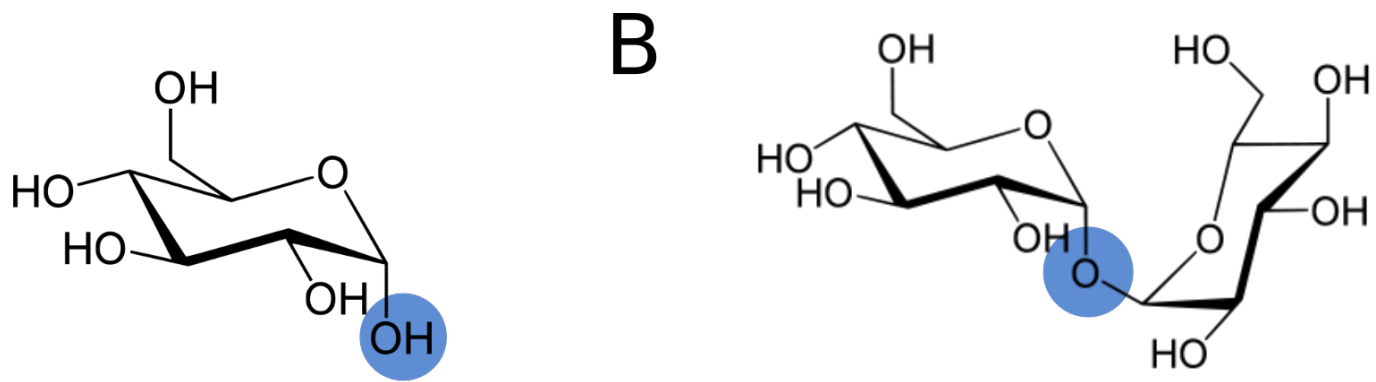

Fig. 1.7 Trehalose and its building blocks. (A) Monosaccharide $\alpha$-D-glucose; the hydroxyl group of the hemiacetal which takes part in forming the chemical bond in trehalose, is highlighted in blue circle. (B) Disaccharide trehalose. Highlighted glycosidic bond is created by joining hemiacetal hydroxyl groups of two $\alpha$,D-glucoses. 


\subsubsection{Cryopreservation with trehalose}

Trehalose has been added in some protocols as a supplement to DMSO or EG, resulting in improvements in plating efficiency, proliferation, albumin and urea formation of hepatocytes [128] or viability of bovine embryos [129]. Others that attempted to use trehalose as the only cryoprotectant, have faced the necessity for intracellular delivery of the disaccharide $[54,130]$. Several studies have suggested that $200 \mathrm{mM}$ concentration of trehalose delivers the highest protective effect [54, 128].

Trehalose has also been investigated for cryopreservation of haematopoietic progenitors. Using the immature haematopoietic cell line TF-1, Buchanan et al. showed that trehalose is a suitable cryoprotectant for HSCs as it preserves their stem cell phenotype and clonogenic potential [54]. Zhang and colleagues demonstrated improvements in colony forming ability of UCB cells if trehalose was added to conventional cryopreservation medium [131]. Similar results were observed by Limaye et al. who cryopreserved HSCs from UCB and foetal liver in $10 \%$ DMSO supplemented with trehalose [132]. Scheinkonig and colleagues investigated using trehalose as the sole cryoprotectant. Although the viability of cells from bone marrow and peripheral blood cryopreserved in trehalose did not reach that of DMSO-cryopreserved cells, the number of colony forming units was higher in trehalose [130].

Cryopreserved islets demonstrated high viability and in vivo survival when the disaccharide was added to the standard cryopreservation solution simultaneously with DMSO [83]. Another study reported that addition of to standard DMSO freezing protocol improved cryosurvival of hydrogel-encapsulated islets [133]. Modified Kyoto solution containing trehalose as a cryoprotectant has been suggested as a preservation solution for human islet isolation that may be superior to University of Wisconsin (UW) solution [134].

\subsubsection{Intracellular trehalose delivery}

Trehalose should be delivered intracellularly to achieve maximal cryoprotection $[54,135$, 136]. However, mammalian cell membranes do not have a transporter for trehalose and uptake by passive diffusion only facilitates intracellular delivery of small quantities of the disaccharide [114]. Several approaches have been explored to increase the intracellular uptake of trehalose, including administration of genetically engineered pores into the membrane [54, 135], microinjections [137], electroporation [138], induction of thermal shock [139], delivery during membrane phase transition [83], addition of trehalose-containing liposomes [136], and using engineered trehalose conjugated to lipophilic groups [140]. A group of amphipathic polymers capable of integrating into the membrane and changing its permeability has also been developed and shown to permit trehalose uptake by erythrocytes and SAOS-2 cells 
(human osteosarcoma cell line) [141, 142], as discussed below. All these methods have their advantages and disadvantages in different cellular and multicellular systems which were reviewed by Stewart et al. [143].

\subsection{Permeabilising polymers}

\subsubsection{Characteristics}

PP-50 is an amphipathic pseudo-peptidic polymer developed by the Department of Chemical Engineering and Biotechnology at the University of Cambridge. It is synthesised by grafting L-phenylalanine (containing an aromatic group) onto a hydrophobic poly(L-lysine isophthalamide) (PLP) backbone via the pendant, weakly ionisable carboxylic acid moieties (Figure 1.8) [144]. The polymer undergoes a conformational change in different $\mathrm{pH}$ : the molecule is extended at higher $\mathrm{pH}$ than its $\mathrm{pKa}$ and folded to a globular structure at lower $\mathrm{pH}$. Such hydrophobically-stabilised structure disrupts the cell membrane and allows the uptake of molecules, such as drugs or trehalose, to which the cell membrane is normally impermeable. These $\mathrm{pH}$-dependent conformational changes also enable removal of the polymer from the membrane by increasing the $\mathrm{pH}$ [141]. Lynch and colleagues have proposed a precise mechanism of membrane disruption, in which membrane thinning localised around the polymer incorporated into the membrane allows more frequent formation of gaps which facilitates trehalose uptake [125].

PP-50 belongs to a whole family of $\mathrm{pH}$ responsive amphipathic polymers with membrane permeabilising ability based on the PLP backbone. Potential modifications include the amino acid grafted (L-valine in PV series, L-leucine in PL series and L-phenylalanine in PP series) and the degree of substitution (from approximately 30 to $65 \%$ ). Composition of some of the polymer variants is summarised in Table 1.3 [141]. These polymers are also biodegradable [145].

Table 1.3 Composition of PLP and examples of its amino acid-grafted variants

\begin{tabular}{lccccccc}
\hline Polymer & PLP & PV-50 & PL-50 & PP-30 & PP-50 & PP-60 & PP-75 \\
\hline Molar ratio* $(\%)$ & 0 & 50.0 & 50.0 & 30.0 & 50.0 & 60.0 & 75.0 \\
Degree of substitution** $(\%)$ & 0 & 46.9 & 46.9 & 32.4 & 46.2 & 54.6 & 63.2 \\
\hline
\end{tabular}

Adapted from Lynch et al. [141]; *Molar ratio of amino acid to carboxylic moieties on PLP used during synthesis; **Actual degree of substitution after synthesis (number of aminoacids grafted per 100 carboxylic acid groups) 


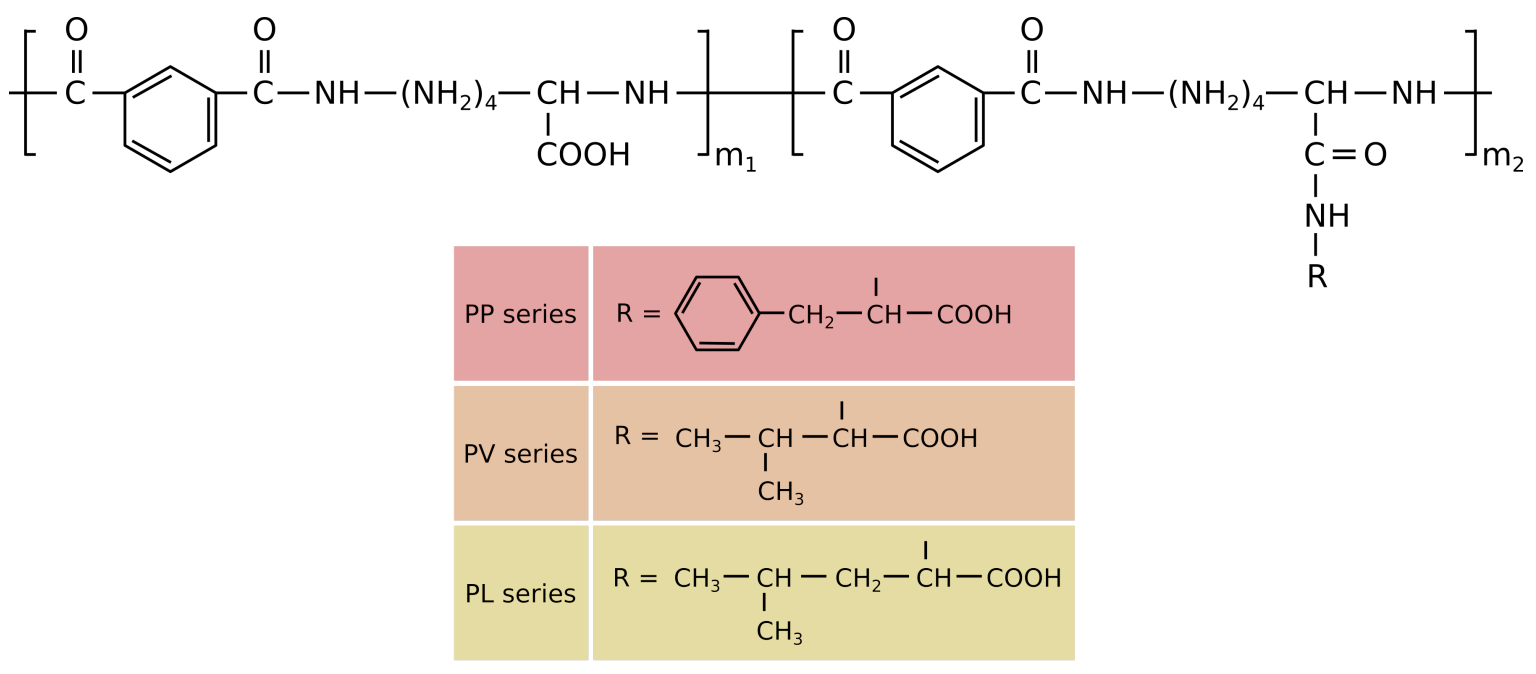

Fig. 1.8 Chemical structure of PLP and its variants. Chemical formula of the synthesised poly(L-lysine isophthalamide) (PLP) backbone containing ungrafted repeat units (with index $\mathrm{m}_{1}$ ) and repeat units grafted with aminoacids (index $\mathrm{m}_{2}$ ) . Aminoacids grafted onto the backbone were L-phenylalanine (PP series, including PP-50 and PP-75), L-leucine (PL series) or L-valine (PV series) (figure adapted from Chen et al. [144])

\subsubsection{PP-50 efficacy and toxicity}

Phenylalanine is the most hydrophobic amino acid at $\mathrm{pH} 7.0$ and, compared to grafting with L-lysine and L-leucine, gives the polymer the most optimal properties for permeabilising the membrane after grafting onto the PLP backbone [144]. Moreover, PP-50 was found to be more effective than PL-50 and PV-50 in loading of trehalose into ovine erythrocytes. The minimal degree of L-phenylalanine substitution in PP series for effective trehalose loading was shown to be $32.4 \%$ [141].

Several studies tested the potential toxicity of the amphipathic polymers on erythrocytes or nucleated cell lines. Toxicity of PLP, PL-75 and PP-75 was evaluated by propidium iodide (PI) staining after $48 \mathrm{~h}$ incubation of HeLa cells with concentration of the polymers from 0 to $2000 \mu \mathrm{g} / \mathrm{mL}$. In this assay, PLP did not result in in vitro toxicity within the concentration range and PL-75 and PP-75 were toxic at concentrations higher than 500 and $50 \mu \mathrm{g} / \mathrm{mL}$ respectively [144]. PP-50 did not decrease metabolic activity of SAOS-2 cells measured by MTS assay up to the concentration $200 \mu \mathrm{g} / \mathrm{mL}$ when incubated for 2 or $24 \mathrm{~h}[142]$. 


\subsubsection{Trehalose and permeabilising polymers}

Few studies have examined intracellular delivery of trehalose mediated by membranedisrupting biopolymers. Lynch et al. showed that intracellular trehalose loading by PP-50 increased cryosurvival of ovine erythrocytes compared to extracellular trehalose alone [141]. Trehalose loading into ovine erythrocytes facilitated by PP-50 has been shown to be dependent on $\mathrm{pH}$, incubation time, temperature, and PP-50 and trehalose concentrations. Efficient loading was only possible at $\mathrm{pH}$ lower than 7.05. Increase in PP-50 concentration led to a rise in intracellular trehalose concentration following incubation, reaching the maximum trehalose permeability at $100 \mu \mathrm{g} / \mathrm{mL}$ of PP-50. Intracellular trehalose concentration reached its maximum if $360 \mathrm{mM}$ extracellular solution of trehalose was used with PP-50; it was suggested that higher concentrations of trehalose could inhibit PP-50 interactions with the membrane. The highest loading of trehalose was observed at a higher incubation temperature $\left(37^{\circ} \mathrm{C}\right)$ and a longer incubation time $(9 \mathrm{~h})$ [141].

SAOS-2 cell line was also used to assess the efficacy of PP-50 in cryopreservation with trehalose to extend the work to nucleated cells. Sharp et al. reported that addition of $\mathrm{PP}-50$ to trehalose cryopreservation medium increases cell recovery. The optimal PP-50 concentration for SAOS-2 cell line was $25 \mu \mathrm{g} / \mathrm{mL}$ with optimal osmolarity of the solution at $133 \mathrm{mOsm} / \mathrm{L}$ [142]. Mercado et al. reported co-localisation of fluorescent PP-50 and fluorescent trehalose in the supra-nuclear space in SAOS-2 cells, suggesting that PP-50 mediated this uptake of trehalose. While addition of PP-50 significantly improved cryosurvival of SAOS-2 cells frozen with $200 \mathrm{mM}$ trehalose, this was still well below the efficacy of the standard $10 \%$ DMSO cryopreservation protocol [146].

Cryopreservation of primary cells, including human haematopoietic progenitors or pancreatic islets with trehalose and permeabilising polymers has not been previously studied.

\subsection{Antioxidants}

\subsubsection{Oxidative stress in cryopreservation}

All cells can tolerate limited exposure to the reactive oxygen species (ROS) that are naturally generated during the process of oxidative phosphorylation and can even be key to the homeostatic metabolic function, signalling of cells or fighting infections. ROS also arise as a consequence of ionising radiation, ischemia, and cryopreservation. Cells express endogenous antioxidant enzymes in amounts sufficient to protect them from damage during homeostasis. However, when the antioxidant capacity of cells is exceeded, redox homeostasis fails and cells are subjected to potentially damaging amounts for ROS [147]. This can have a detrimental 
impact on the mitochondrial health, integrity of DNA, function of proteins in cells and ultimately lead to apoptosis or malignant transformation [148].

The cryopreservation process inherently causes stress to cells and it may be necessary to target multiple pathways to prevent apoptosis and ROS-related damage to DNA, proteins and other cellular components. ROS can arise in cryopreserved tissue for multiple reasons. Firstly, it has been shown that osmotic stress, which is to some extent always present during cryopreservation due to addition of cryoprotectants, can increase production of ROS. Additionally, thawed cells initiate many repair mechanisms which increases the oxidative metabolism, generating more ROS [149]. Majority of antioxidants previously tested in cell cryopreservation showed improvement in the post-thaw cell quality but there is also evidence for antioxidant resveratrol that while its addition decreased the oxidation of cellular components, it did not achieve an improvement in function of cells (in this case, the sperm motility) [150].

Pancreatic islet cells have decreased amounts of endogenous antioxidant enzymes (catalase, glutathione peroxidase and superoxide dismutase) compared to other tissues such as liver, which makes them more sensitive to increased levels of ROS [127]. Addition of variety of antioxidant compounds during the isolation and culture of islets before transplantation has been tested with encouraging results [151]. Increased levels of ROS are found during the progression of type 2 diabetes and antioxidants have been shown to prevent hyperglycaemia and other markers of diabetes [152]. Addition of exogenous antioxidants may therefore tilt the balance towards survival during pancreatic islet cryopreservation.

\subsubsection{Salidroside}

Positive effect of natural plant-derived compounds on pancreatic islet survival and insulin secretion has been well demonstrated [153]. Even in cryopreservation, the extract from Curcuma longa called curcumin has been shown to improve the post-thaw viability and insulin secretion profile in pancreatic islets [154]. Another natural antioxidant, salidroside, is a promising candidate for improvement of cryopreservation outcome but has not yet been tested either for islet culture or cryopreservation.

Salidroside (Figure 1.9A) is an active compound of the medicinal herb Rhodiola rosea with demonstrated pharmacological effects [155]. Al-Otaibi et al. have shown that salidroside improves cryopreservation of red blood cells by positively affecting their oxidative status, manifested as a decrease in lipid peroxidation and protein carbonylation [156]. Similarly, decreased lipid oxidation and lactate dehydrogenase activity, as well as increased expression of proteins involved with DNA repair and cell proliferation were demonstrated for human leukaemia cell line cryopreserved with combination of DMSO and salidroside [157]. Safety 


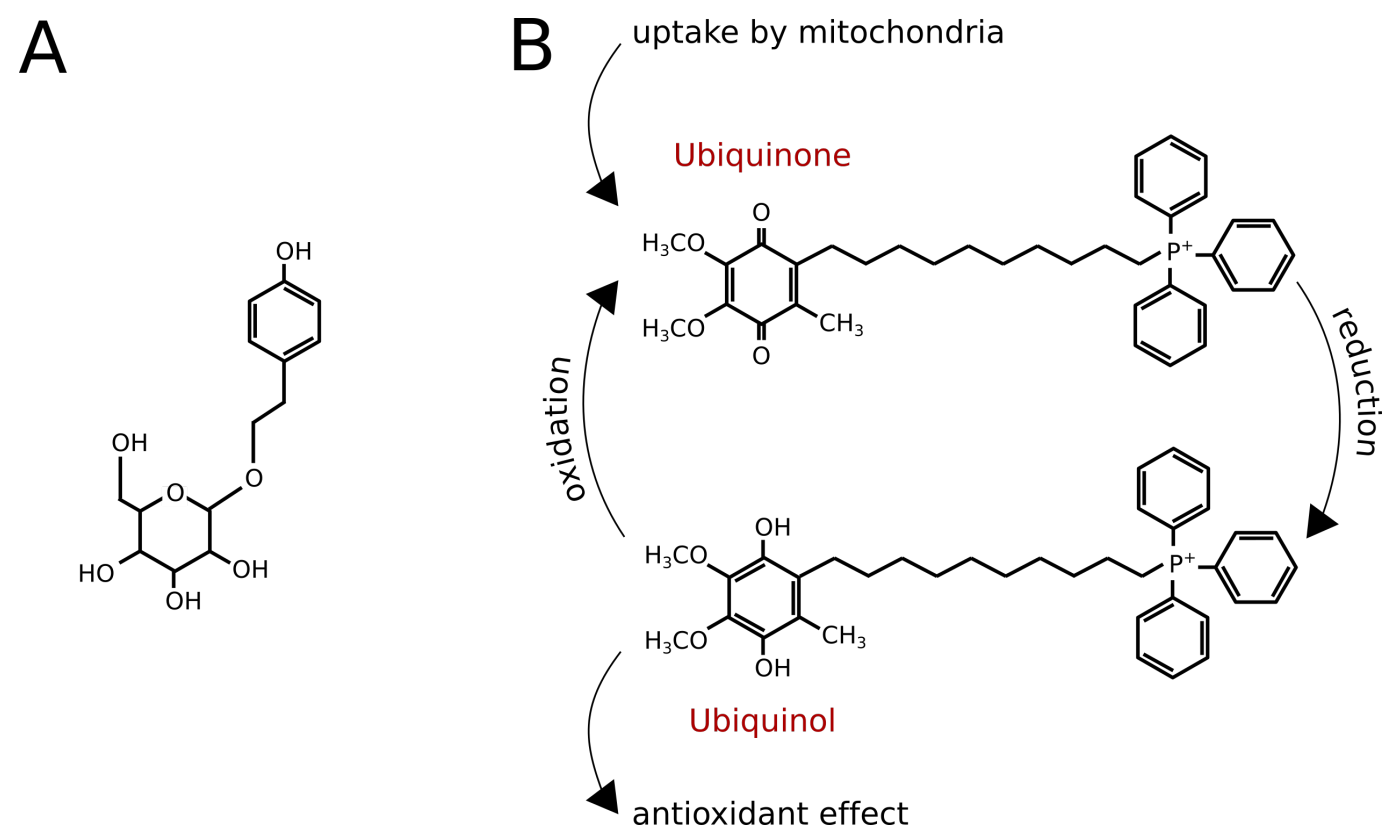

Fig. 1.9 Structure of antioxidants salidroside and MitoQ. (A) Chemical structure of salidroside (B) Chemical structure of MitoQ and changes happening during oxidation and reduction.

of orally administrated $R$. rosea has also been demonstrated in multiple clinical trials [158]. Although the precise mechanism behind its anti-oxidative properties is currently unclear, it is known that in red blood cells, salidroside targets signalling pathways involved in induction of apoptosis and prevents activation of caspase- 3 and increase in intracellular $\mathrm{Ca}^{2+}$ ions [155].

\subsubsection{MitoQ}

The majority of ROS in cells is generated during the reactions of mitochondrial respiratory chain. Increased concentration of ROS in mitochondria causes oxidation of mitochondrial components (mtDNA, lipids, proteins) which can then initiate a cascade of events leading to apoptosis and ultimately the death of the cell [148].

Mitoquinone (MitoQ) is a mitochondria-targeted antioxidant composed of a lipophilic triphenylphosphonium cation and antioxidant ubiquinone. The lipophilic part of the molecule directs the transport of MitoQ to mitochondria, where it accumulates and therefore delivers the antioxidative effect directly at the main source of ROS. Upon entrance into mitochondria, the oxidised ubiquinone form is reduced by complex II of the respiratory chain to ubiquinol which can then exhibit its antioxidant function by being oxidised back to ubiquinone. It can then be repeatedly recycled back to the original ubichinol form which ensures that each molecule can exhibit its effect many times (Figure 1.9B) [159]. 
MitoQ has been shown to decrease oxidative damage during ischemia reperfusion injury in kidney and heart $[160,161]$. Two clinical trials have been performed using MitoQ for the treatment of hepatitis C [162] and Parkinson's disease [163] and demonstrated safety of MitoQ. Additionally, MitoQ was shown to provide protection against oxidative stress in $\beta$-cell lines in the context of type 2 diabetes progression [164]. It has been previously tested in the context of cryopreservation in sperm [165] and heart valve tissue [166], suggesting that it is a good candidate for ameliorating oxidative stress in cryopreservation. It has never been tested for cryopreservation of pancreatic islets.

\subsection{Project aims}

The ultimate aim of this project was to improve cryopreservation methods for pancreatic islets for the purposes of transplantation by investigating potential of novel compounds including disaccharide trehalose, permeabilising biopolymers PP-50 and PP-75 and antioxidants MitoQ and salidroside. Optimisation of culture, viability assessment and performance of these compounds in a single-cell model are important steps leading to the final cryopreservation method for cell clusters.

The specific aims of this project were to:

1. Improve the methods of viability assessment of pancreatic islets for the purposes of cryopreservation research and transplantation

2. Use mononuclear cells from deceased organ donors as a single cell model for investigation of the efficacy of trehalose and membrane-permeabilising polymers

3. Investigate the potential of trehalose, permeabilising polymers and antioxidants for cryopreservation of pancreatic islets:

(a) In a novel DMSO-free method

(b) As additives to the existing DMSO-based method 


\subsection{Experimental approach}

To improve viability assessment in both mononuclear cells and pancreatic islets, the key step was identification of the limitations of current methods. These were demonstrated using a series of experiments and approaches to improve them were suggested.

Because function of permeabilising polymers has never been explored in primary cells, mononuclear cells were used for optimisation of its function before proceeding to multicellular islet model. All mononuclear cell experiments were performed using human cells from deceased human organ donors. Screening of methods for pancreatic islet cryopreservation was performed using mouse islets and where possible, replicated using deceased human organ donor islets.

To select suitable cryopreservation protocols, a variety of cryopreservation protocols, cryoprotectant combinations and additives were systematically screened using flow cytometry for mononuclear cells and live/dead staining and wide-field fluorescent microscopy for pancreatic islets. Slow freezing was chosen over vitrification due to disadvantages of vitrification including use of toxic concentrations of cryoprotectants and the related difficulty of removing these from multicellular aggregates. Slow-freezing has generally been better explored than vitrification and standard protocol exists which can be modified with available technologies. The modifications of the cryopreservation methods considered in this project include:

- Pre-cryopreservation culture conditions

- Culture medium composition and culture temperature

- Composition of cryopreservation medium

- Presence of DMSO

- Addition of trehalose

- Addition of permeabilising polymers for intracellular trehalose delivery

- Addition of antioxidants

- Concentration of all components (cells, DMSO, additives)

- Incubation of cells with the cryoprotectants

- Method of cryoprotectant addition

- Incubation time

- Incubation temperature 
- Freezing protocol

- Freezing rate

- Thawing protocol

- Thawing rate

- Removal of cryoprotectant

The successful protocols selected by this optimisation were then validated using variety of in vitro functional assays and in vivo testing in mice. With pancreatic islets, the focus was to evaluate the function of critical $\beta$-cell pathways described in Chapter 1.2.2 (by glucosestimulated insulin secretion and cAMP production after stimulation with incretin hormones), mitochondrial health (mitochondrial DNA oxidation and ATP/ADP ratios) and ability to survive and function in vivo (transplantation into diabetic mice). 


\section{Chapter 2}

\section{Materials and Methods}

\subsection{Tissues from deceased human organ donors}

\subsubsection{Tissue retrieval}

Tissues from deceased human organ donors after circulatory death (DCD) or brainstem death (DBD) were collected under ethical approval (REC Ref: 15/EE/0152) after obtaining informed consent for use in research from the donor family. Deceased donors are a potential alternative source of haematopoietic progenitors suitable for transplantation as they retain their function even after certain periods of hypoxia [27, 167].

Bone marrow (BM) was aspirated from vertebrae using an $11 \mathrm{G}$ bone marrow biopsy needle (Jamshidi ${ }^{\mathrm{TM}}$ ) and collected into a heparinised $50 \mathrm{~mL}$ syringe. Multiple pieces of spleen were excised and placed on ice in a container filled with Belzer UW®Cold Storage Solution (Bridge to Life Ltd.) within one hour of circulatory arrest. Some of the spleens were obtained as attached to pancreases intended for transplantation and subsequently declined by multiple transplant centres (under ethical approval REC Ref: 12/EE/0253, substituted by 16/EE/0227). Notably, such spleen tissue will have spent a prolonged period in cold preservation solution before being ready for processing in the laboratory $(12-18 \mathrm{~h})$. BM was processed and cryopreserved within $24 \mathrm{~h}$ after receipt and the spleen within $72 \mathrm{~h}$ after receipt.

\subsubsection{Processing tissues}

\section{Spleen}

Spleen was cut into small pieces of approximately $1 \mathrm{~cm}^{3}$ using a scalpel and transformed into a single cell suspension by one of the following two methods. 
In the first method, the tissue was pushed through a $100 \mu \mathrm{m}$ sieve using a syringe plunger, until it was completely disintegrated, and the sieve was then washed with Dulbecco's Modified Eagle Medium (DMEM, Thermo Fisher Scientific) supplemented with 10 \% FCS (Sigma Life Sciences). Second method utilised the gentleMACS ${ }^{\mathrm{TM}}$ Dissociator (Miltenyi Biotec). Sections of spleen were placed into gentleMACS ${ }^{\mathrm{TM}} \mathrm{C}$ Tubes (Miltenyi Biotec) and covered with DMEM $+10 \%$ FCS. Tissue was dissociated into a single-cell suspension using a pre-set programme on the device.

Cell suspension from either method was filtered through a $70 \mu \mathrm{m}$ cell strainer and mononuclear cells were isolated by density gradient separation. $20 \mathrm{~mL}$ of the cell suspension was carefully layered on top of $15 \mathrm{~mL}$ of Lymphoprep ${ }^{\mathrm{TM}}$ (Stemcell Technologies) in a $50 \mathrm{~mL}$ Falcon tube. The tubes were then centrifuged at $800 \mathrm{~g}$ for $25 \mathrm{~min}$ at $20^{\circ} \mathrm{C}$ with the brake off. The layer of mononuclear cells was collected by Pasteur pipette and washed twice by DMEM $+10 \%$ FCS, centrifuged at $300 \mathrm{~g}$ for $7 \mathrm{~min}$ with the brake on. Cells were then counted using haemocytometer and resuspended in a cryopreservation medium for freezing or in culture medium for analysis of fresh sample.

\section{Bone marrow}

Bone marrow was filtered through a $70 \mu \mathrm{m}$ cell strainer, separated on density gradient media and further processed as described above for spleen.

\subsubsection{Obtaining human pancreatic islets}

Pancreatic islets were isolated from pancreases of human deceased organs donors by SNBTS Islet Cell Laboratory in Edinburgh (ethical approval REC Ref: 16/WM/0093). These clinically isolated islets were declined for transplantation due to low purity, viability or yield and subsequently offered for research use. They were shipped in CMRL 1066 Supplemented culture media (Corning) at room temperature and further processed upon arrival. The purity and viability of the received islets was validated upon receipt in our laboratory using dithizone staining and fluorescein diacetate/propidium iodide staining as described in Sections 2.6.1 and 2.6.2.

\subsubsection{Information storage}

Anonymised donor information was retrieved from a database managed by research assistants at the Department of Surgery. A dedicated database of samples stored in the liquid nitrogen dewar was managed and regularly updated, ensuring that manipulation of samples in the dewar by all users was kept to a minimum. 


\subsection{Animals and animal procedures}

\subsubsection{Animal provenance and husbandry}

All animals were housed and maintained in University Biomedical Services facility under specific-pathogen-free conditions. All procedures were performed under project licences (PPL numbers 70/8702 and 80/2638) approved by the United Kingdom Home Office under the Animal (Scientific Procedures) Act 1986.

Male C57BL/6JAX mice were purchased from Charles River, UK and subsequently used for isolation of mouse pancreatic islets as described in Section 2.2.2. These mice were maintained following standard husbandry procedures in the animal facility and used for experiments between the age of 5 and 12 weeks.

Fully immunodeficient NSG (NOD.Cg-Prkdc ${ }^{\text {scid }}$ IL $2 \mathrm{rg}^{\mathrm{tm} 1 \mathrm{Wjl}} / \mathrm{SzJ}$, NOD scid gamma) mice, which lack B, T and NK lymphocytes [168] were bred in-house, litters weaned at three weeks of age and used for experiments between 6 and 13 weeks of age. Maintenance of this strain was performed with special care, reflecting its immunocompromised background (weekly cage changes and daily checks prior to other mouse strains, aseptic technique).

\subsubsection{Isolation of mouse pancreatic islets}

C57BL/6JAX mice were sacrificed by neck dislocation schedule 1 method. In a supine position (head facing the surgeon), laparotomy was performed and diaphragm cut though and fixed with a tape to allow space for the liver to be flipped over and uncover the gall bladder and bile duct. Bile duct leads though the head of the pancreas and enters the duodenum at hepato-pancreatic ampulla (white patch on the duodenum). Two clamps were placed on the bowel, one on each side of the ampulla to prevent leaking of the perfusate into the bowel (Figures 2.1A and B). Solution of collagenase XI (2.5 mL, Sigma Life Science, $1000 \mathrm{U} / \mathrm{mL}$ in HBSS medium) was slowly injected into the bile duct, starting from the gall bladder, using a $2.5 \mathrm{~mL}$ syringe with a $30 \mathrm{G}$ beveled needle. Once the volume was injected, the pancreas lobules were clearly separated by the injected solution (Figure 2.1C). Pancreas was then carefully dissected out of the abdomen, placed into a $50 \mathrm{~mL}$ Falcon tube containing another $2 \mathrm{~mL}$ of collagenase XI solution and kept on ice until pancreases from all animals were retrieved and tissue transferred into laboratory.

In the laboratory, Falcon tubes with the pancreases were incubated in $37{ }^{\circ} \mathrm{C}$ water bath and shaken every 5 min until the tissue was digested into a fine suspension (typically for 12 to $18 \mathrm{~min}$ ). Digestion was then terminated by adding $25 \mathrm{~mL}$ of ice-cold solution of HBSS supplemented with $1 \mathrm{mM} \mathrm{CaCl}_{2}$ into each tube and centrifuging at $300 \mathrm{~g}$ for $1 \mathrm{~min}$ at $4{ }^{\circ} \mathrm{C}$. 
Another two washes with $\mathrm{HBSS}+\mathrm{CaCl}_{2}$ were performed and the digest in each tube was then resuspended in $10 \mathrm{~mL}$ Histopaque 1077 Hybri Max $^{\mathrm{TM}}$ (Sigma Aldrich) at room temperature. RPMI medium ( $5 \mathrm{~mL}$, at room temperature) was carefully layered on top of the Histopaque and tubes were centrifuged (Figure 2.1D). After centrifuging, the islet-enriched layer can be found on the interface of Histopaque and RPMI, as shown in Figure 2.1E. To increase the yield, both RPMI and Histopaque layers were collected into a new Falcon tube, topped up with RPMI and centrifuged at $300 \mathrm{~g}$ for $3 \mathrm{~min}$. The pellet was transferred into a $10 \mathrm{~mm}$ Petri dish. Although the density gradient separation yielded reasonably pure fraction of pancreatic islets if assessed by dithizone staining, full purification was achieved by manual handpicking of islets under inverted microscope (Figures $2.1 \mathrm{~F}$ and $\mathrm{G}$ ).

Single pancreas yielded approximately 150 islets. These were subsequently cultured in RPMI $+10 \% \mathrm{FCS}+1 \%$ Penicilin/Streptomycin at $37{ }^{\circ} \mathrm{C}$ and $5 \% \mathrm{CO}_{2}$ in humidified atmosphere for 1 to 7 days before being used for further experiments. Viability of islets did not decrease over this period (data not shown) and to control the quality of islets, viability staining was always done before the start of any experiment.

\subsubsection{Transplantation of islets under the kidney capsule of NSG mice}

Pancreatic islets were handpicked into one $1.5 \mathrm{~mL}$ Eppendorf tube per each individual injected mouse, centrifuged at $300 \mathrm{~g}$ for $1 \mathrm{~min}$, supernatant removed and islet in each tube resuspended in $30 \mu \mathrm{L}$ of Matrigel (Corning). Samples were kept on ice until the injections to prevent solidification of the Matrigel.

NSG mice were anaesthesised using mixture of isoflurane and oxygen, shaved on the left side and cleaned with chlorhexidine. The animal was placed on a heat mat set to $37^{\circ} \mathrm{C}$, positioned on its side. Small incision in the skin and muscle was made at the location of the kidney. Kidney was then gently pushed outside through this opening and kept from drying out using warmed saline. A small incision was made in the kidney capsule with a sharp $20 \mathrm{G}$ needle. Through this incision, a blunt needle attached to Hamilton syringe containing the Matrigel suspension with islets was carefully pushed through the kidney parenchyma and $30 \mu \mathrm{L}$ of the suspension was injected under the kidney capsule. Syringe was loaded before each injection to prevent differences in number of received islets per mouse.

After removing the needle from the injected kidney, bleeding was stopped by applying mild pressure on the wound with a cotton bud. Once the kidney was returned to the peritoneal cavity, the muscle layer was closed using a continuous 5-0 vicryl sutures and the skin layer with interrupted sutures. To ensure that the animals do not open the wound, two to three autoclips were applied onto the stitches. After the surgery, the animals were placed into 


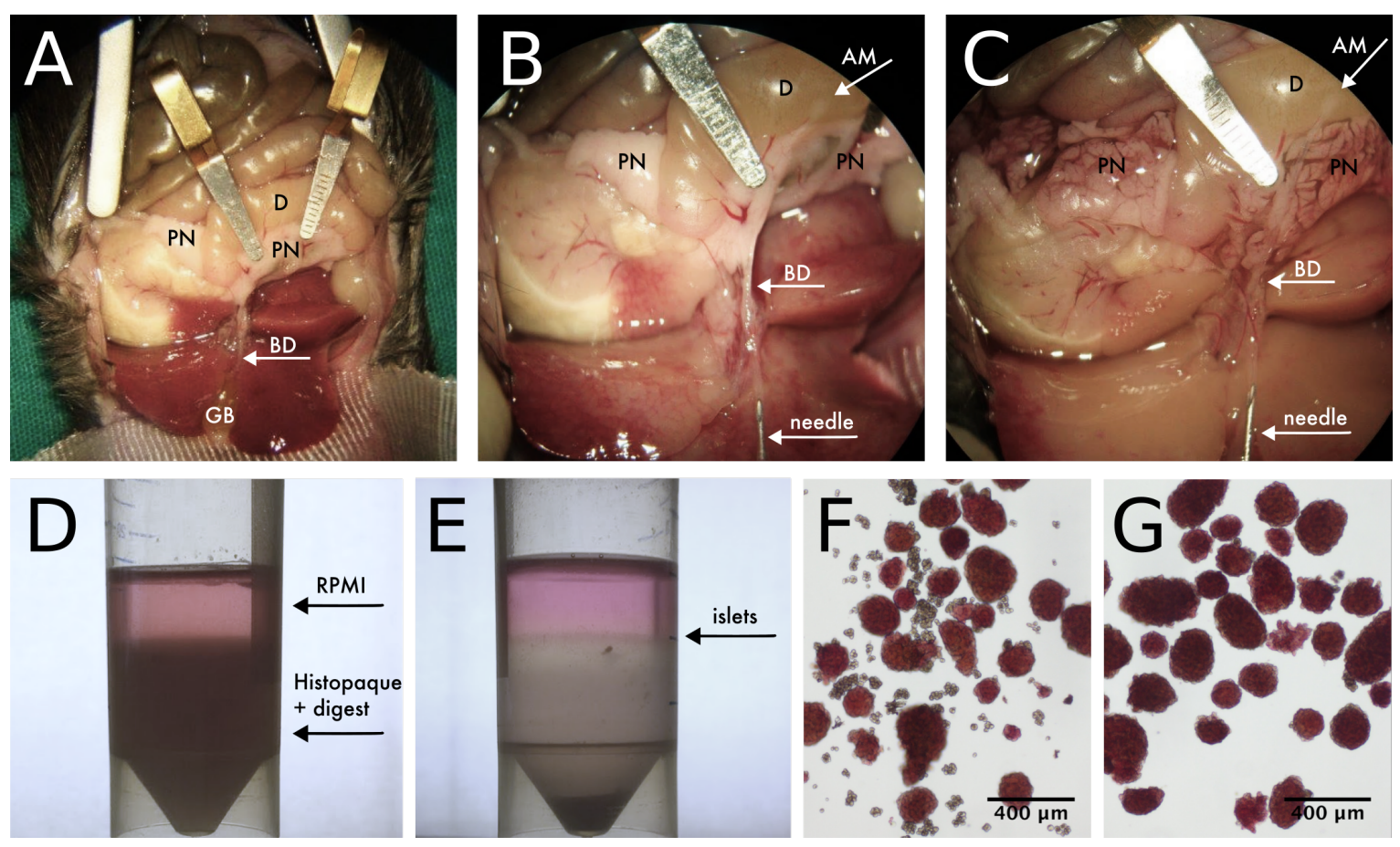

Fig. 2.1 Method of mouse pancreatic islet isolation. (A) Preparation of the animal before injection with collagenase. (B) Close-up of the organs and the injection site before injection. (C) Close-up of the organs and the injection site after injection with visible discolouring of the liver and extension of the pancreas lobules. (D) Tube with the pancreas digest before density gradient separation. (E) Tube with the pancreas digest after density gradient separation. Highlighted is the layer of islets in between Histopaque and RPMI. (F) Microscopic picture of dithizone-stained pancreatic islets before handpicking. Endocrine tissues stain pink while the exocrine acinar cells do not. (G) Microscopic picture of dithizone-stained pancreatic islets after handpicking. $\mathrm{AM}=$ hepato-pancreatic ampulla; $\mathrm{BD}=$ bile duct $; \mathrm{D}=$ duodenum; $\mathrm{GB}=$ gall bladdder; $\mathrm{PN}=$ pancreas .

individual recovery cages and monitored for several hours, then returned into their original cages.

\subsubsection{Induction of diabetes in NSG mice}

Streptozotocin (STZ, Sigma Life Science) solution was prepared on the day of the injection by dissolving STZ powder in freshly prepared citrate buffer $(0.1 \mathrm{M}$ citric acid $+0.1 \mathrm{M}$ sodium citrate, $\mathrm{pH} 4.2$ ) at a concentration of $10 \mathrm{mg} / \mathrm{mL}$ and kept on ice until the injections.

NSG mice were weighed, anaesthesised using mixture of isoflurane and oxygen and injected with $40 \mathrm{mg} / \mathrm{kg} \mathrm{STZ}$ intraperitoneally on five subsequent days using $30 \mathrm{G}$ needle. Higher doses of STZ were initially tested which produced rapid-onset severe diabetes but these methods were abandoned due to a rapid weight loss of the animals (Appendix E). 
In addition to their regular chow, these mice were fed mashed food with the same composition for the duration of the experiment. Weights and blood glucose were measured before the first injection of STZ and subsequently every 3-4 days until the end of the experiment. Blood glucose was measured using iHealth Smart Gluco-Monitoring system (iHealth Labs) by tail vein bleeds.

Mice were considered diabetic when their blood glucose reached over $13.5 \mathrm{mmol} / \mathrm{L}$ on two consecutive measurements and used for transplantation when blood glucose reached over $18 \mathrm{mmol} / \mathrm{L}$. Diabetic animals were transplanted with fresh or cryopreserved islets (or Matrigel as a control) under the kidney capsule (as described in Section 2.2.3) to assess the function of islet cells.

Animals were culled if they lost over $20 \%$ of the weight measured at the start of the experiment or at 4 weeks after transplantation. Blood serum was collected at the start of the experiment, before the islet transplantation and at the endpoint for biochemistry measurements. At the endpoint, blood was collected for serum using inferior vena cava bleed and transplanted kidney removed under terminal anaesthesia, embedded in paraffin and kept for potential immunohistochemical analysis (not included in this thesis).

\subsubsection{Collection and processing of mouse peripheral blood}

\section{Tail vein bleeds for regular glucose measurements}

Mice were gently placed in a restrainer and tail vein was incised using a $30 \mathrm{G}$ needle. Small drop of blood was loaded into the strip of iHealth Smart Gluco-Monitoring system (iHealth Labs) and displayed glucose concentrations were recorded. Glucose meter was validated agains biochemistry measurements in Appendix E). Bleeding was stopped by applying mild pressure.

\section{Tail vein bleeds for collection of serum}

Animals were placed in a $37^{\circ} \mathrm{C}$ warming cabinet for 10 min to allow for dilation of blood vessels. Once warmed, mice were gently placed into a restrainer, tail vein was incised using a $25 \mathrm{G}$ needle and $1-6$ drops of blood were collected from a tail vein into a blood collection tube. Bleeding was then stopped by applying mild pressure.

\section{Inferior vena cava bleeds}

Animals were anesthesised with isoflurane and oxygen and placed on a heat mat set to $37^{\circ} \mathrm{C}$. Laparotomy was performed to reveal the abdominal organs. Bowels and liver were carefully 
moved to the side using a cotton bud to reveal the inferior vena cava (IVC). Using $1 \mathrm{~mL}$ syringe with attached $25 \mathrm{G}$ needle with the bevel facing up, blood was slowly drawn from the top portion of the IVC (typically up to $700 \mu \mathrm{L}$ ). Exsanguination under anaesthesia was a non-recovery procedure used as a killing technique.

\section{Extraction of serum from peripheral blood and glucose quantification}

Blood collected from tail vein or IVC bleeds was kept at $4{ }^{\circ} \mathrm{C}$ for $24 \mathrm{~h}$ after collection, allowing for it to coagulate. Tubes were then spun in a microcentrifuge at $13000 \mathrm{~g}$ for $7 \mathrm{~min}$. Clear serum was collected from the top supernatant layer, transferred into a new Eppendorf tube and stored at $-20{ }^{\circ} \mathrm{C}$ until analysis.

Glucose measurements in mouse serum were done by the Core Biochemical Assay Laboratory (supported by the MRC MDU Mouse Biochemistry Laboratory [MRC MC UU 12012/5]). Serum glucose was measured using a colorimetric assay on the Siemens Dimension EXL autoanalyser. All reagents and calibrators were supplied by Siemens.

\subsection{Compound provision and synthesis}

\subsubsection{Synthesis of PP-50 and PP-75}

The PP-50 and PP-75 polymers were synthesised and kindly provided by Dr. Krishnaa T. Mahbubani and Alex Chen. The synthesis steps are briefly described below.

The polymer backbone (PLMEP) was synthesised by combining potassium carbonate and L-lysine methyl ester dihydrochloride aqueous solution with iso-phthaloyl dichloride solution in acetone. The polymers of varying length were formed at the liquid-liquid interface. After one hour, the formed globule was thoroughly washed from organic substances. It was then dried in a $55^{\circ} \mathrm{C}$ vacuum oven overnight.

The dry globule was ground into a powder and dissolved in DMSO. Solution of sodium hydroxide in ethyl alcohol was then added and incubated for three minutes to allow precipitation. At this step, the methyl groups of PLMEP were substituted by hydroxyl groups. The precipitate was collected by vacuum filtration and its aqueous solution was purified by dialysis which ensures DMSO removal. After seven days of dialysis, the $\mathrm{pH}$ of the purified solution was adjusted to 7.4 by $1 \mathrm{M}$ sodium hydroxide, followed by lyophilisation of PLP.

In the next step, L-phenylalanine was grafted on the PLP backbone in a reaction of PLP with L- phenylalanine methyl ester $\cdot \mathrm{HCl}$, mixed with triethyl amine and 4-dimethylaminopyridine in DMSO/DMF (dimethylformamide) solution. A solution of N,N'-dicyclohexylcarbodiimide in DMF was then drop-wise added to the solution. After 60 h, $5 \%$ sodium 


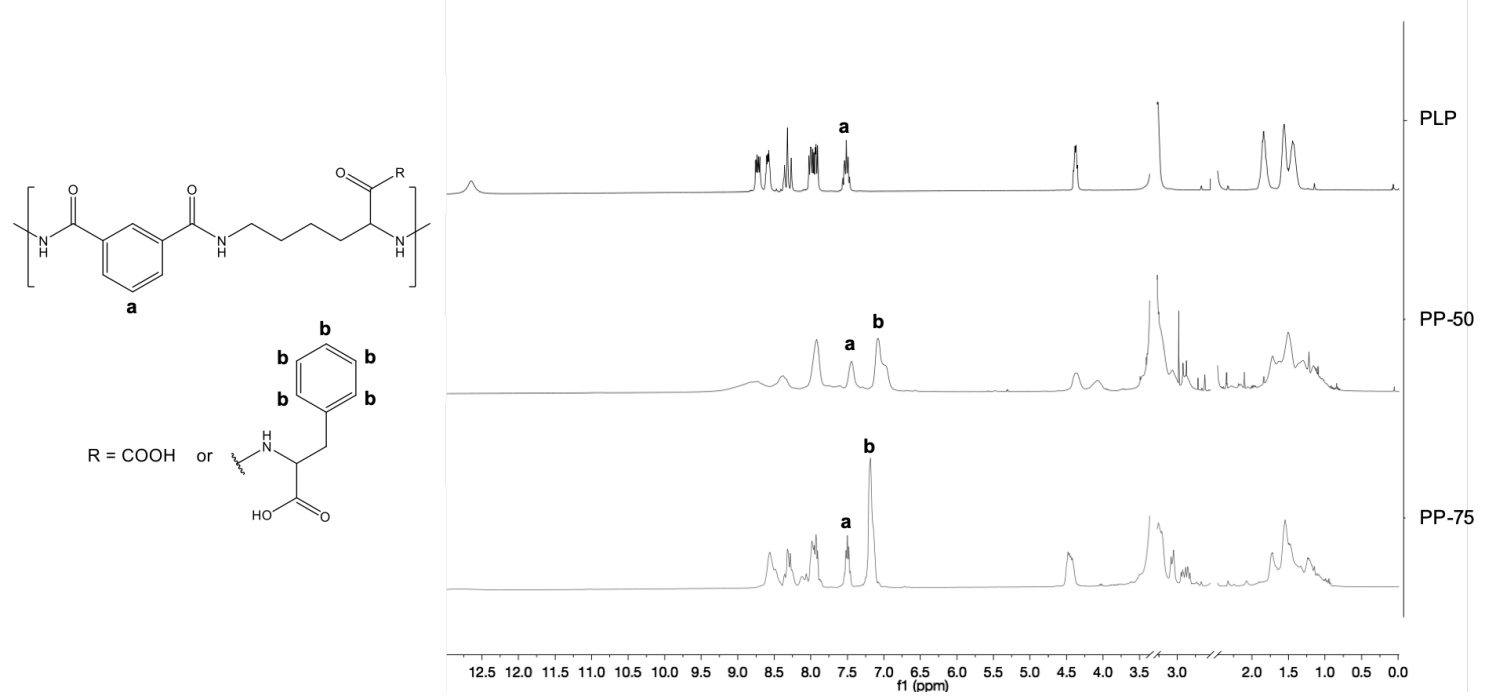

Fig. 2.2 Chemical structure and ${ }^{1} \mathrm{H}-\mathrm{NMR}$ spectra of PLP, PP-50, and PP-75. The unique proton on the backbone benzene was denoted as a and assigned to the according peak on the NMR spectra. Five protons on the phenyl group were denoted as $\mathbf{b}$ and assigned to the peak on the NMR spectra of PP-50 and PP-75. DMSO and $\mathrm{H}_{2} \mathrm{O}$ peaks at 2.5 and $3.5 \mathrm{ppm}$ were removed for better visualisation of the spectra. This figure is a courtesy of Dr. Alex Chen. ${ }^{1} \mathrm{H}-\mathrm{NMR}=$ Proton nuclear magnetic resonance; ppm $=$ parts per million .

hydroxide was added to the reaction mixture to allow precipitation. Again, the precipitate was collected by vacuum filtration and purified by dialysis in distilled water. After ten days, the product was collected, $\mathrm{pH}$ adjusted to 7.4 and it was lyophilised into a fine powder. Prior to use, the PP-50 powder was dissolved in PBS at a concentration $20 \mathrm{mg} / \mathrm{mL}$.

The PLP backbone and the two synthesised polymers were subjected to proton nuclear magnetic resonance $\left({ }^{1} \mathrm{H}-\mathrm{NMR}\right)$ analysis by Dr. Alex Chen and the results are displayed in Figure 2.2. The grafting percentage calculated by ${ }^{1} \mathrm{H}-\mathrm{NMR}$ was $46.0 \%$ and $60.8 \%$ for PP-50 and PP-75 respectively.

\subsubsection{Fluorescent labelling of PP-50}

Fluorescent labelling of PP-50 was done with kind help of Dr Krishnaa T. Mahbubani. First, PP-50 was dissolved in mixture of DMSO and distilled water. N-(3-dimethylaminopropyl)$\mathrm{N}$ '-ethylcarbodiimide hydrochloride (EDC) and N-hydroxysuccinimide (sulfo-NHS) were dissolved in distilled water. The EDC/Sulfo-NHS mixture was added to the PP-50 solution and left to react for one hour at room temperature. Alexa Fluor ${ }^{\circledR} 647$ (AF647) cadaverine (Thermo Fisher Scientific) was dissolved in $1 \mathrm{M} \mathrm{Na}_{2} \mathrm{CO}_{3}$ and added to the activated PP50/EDC/Sulfo-NHS mixture to react at room temperature overnight in the dark. 
On the next day, $5 \%$ DMSO in PBS was added to the solution to dissolve any precipitate. The solution was then dialysed against distilled water for 1 week in 3500 Da tubing with daily water changes. After one week, the remaining solution was transferred to a $50 \mathrm{~mL}$ Falcon tube and concentrated $\mathrm{HCl}$ was added to precipitate the AF647-labelled PP-50. Mixture was then centrifuged at $2500 \mathrm{~g}$ for $3 \mathrm{~min}$ and supernatant centrifuged again to recover any remaining precipitate. Blue solid was then dissolved using $\mathrm{NaOH}$ and $\mathrm{PBS}$, dialysed again against distilled water for $3 \mathrm{~h}$ and subsequently lyophilised to yield a blue solid powder. The powder was then dissolved in PBS at concentration of $2 \mathrm{mg} / \mathrm{mL}$ prior to use.

\subsubsection{Provision of antioxidants}

MitoQ was kindly provided by Prof. Michael P. Murphy in the powder form. The batch was synthesised in 2012. Prior to use, the powder was dissolved in ethanol at concentration of $50 \mathrm{mM}$ and further diluted with ethanol to make a working stock of $50 \mu \mathrm{M}$. Powder and dissolved MitoQ and stock solutions were stored at $-20{ }^{\circ} \mathrm{C}$.

Salidroside was purchased from Sigma Aldrich. Stock solution was made by diluting the salidroside powder in DMSO at $200 \mathrm{mM}$ concentration. Powder form and stock solutions were stored at $-20{ }^{\circ} \mathrm{C}$.

\subsection{Cryopreservation}

\subsubsection{Cryopreservation of mononuclear cells}

Mononuclear cells from spleen, bone marrow or peripheral blood were frozen in $1.8 \mathrm{~mL}$ cryovials (Thermo Fisher Scientific) in a final volume of $1 \mathrm{~mL}$ under different experimental conditions. These included DMSO control and various combinations of trehalose (solutions made from D-(+)-trehalose dihydrate, Sigma Life Sciences) and PP-50/PP-75 polymers in DMEM medium with or without FCS. Other variations included addition of incubation period or changing concentrations of the components. Cryovials were then transferred to a freezing container (Nalgene) and placed in a $-80{ }^{\circ} \mathrm{C}$ freezer overnight. The samples were then transferred into a liquid nitrogen dewar for at least one day before the thawing and analysis.

\subsubsection{Thawing of mononuclear cells}

The thawing procedure was optimised during a series of experiments for different cryopreservation solutions (see Results section 4.3.3), the protocol described here is the final optimised 
protocol. Sample was removed from the liquid nitrogen dewar onto dry ice and transferred to a $37{ }^{\circ} \mathrm{C}$ water bath. Samples frozen down with DMSO were kept in the water bath only until a small ice crystal was left, whereby the trehalose samples were incubated for $10 \mathrm{~min}$. The suspension was then transferred into a $50 \mathrm{~mL}$ Falcon tube. The thawing media, consisting of DMEM + $50 \%$ FCS and pre-warmed to $37^{\circ} \mathrm{C}$, was added slowly drop-wise to the tube up to the volume of $20 \mathrm{~mL}(20 \times$ the original volume). The tube was then centrifuged at $300 \mathrm{~g}$ for $5 \mathrm{~min}$. The cells were resuspended and counted using haemocytometer. Cells were then ready for further analysis.

\subsubsection{Cryopreservation of pancreatic islets}

Human or mouse pancreatic islets were handpicked into a $1.8 \mathrm{~mL}$ cryovial and cryoprotectant was added according to the experimental protocol. Standard cryopreservation protocol consists of cryopreservation in 2 M DMSO in RPMI (mouse islets) or CMRL (human islets) with $10 \%$ FCS. Addition of 2 M DMSO was optimised (see Section 5.3.1) and the final protocol consists of three additions of DMSO over $45 \mathrm{~min}$ to gradually increase its concentration ( $5 \mathrm{~min}$ in $0.66 \mathrm{M}$ DMSO at room temperature, $25 \mathrm{~min}$ in $1 \mathrm{M} \mathrm{DMSO}$ at room temperature and $15 \mathrm{~min}$ in $2 \mathrm{M} \mathrm{DMSO}$ at $4{ }^{\circ} \mathrm{C}$ ). Any variations to this standard protocol are described in the Results section. Additionally, compounds such as trehalose (D-(+)-trehalose dihydrate, Sigma Life Sciences), MitoQ or salidroside (Sigma Aldrich) were used during the incubation period before the addition of DMSO. Details of cryoprotectant combinations are described in the Results section.

Freezing was performed using the CRF-1 controlled rate freezer (Grant) set to a programme described in Table 2.1. Briefly, islets were brought to $-7.4{ }^{\circ} \mathrm{C}$ and held at that temperature for $15 \mathrm{~min}$ to allow for nucleation and release of latent heat of fusion. Following this, islets were slow-frozen to $-40{ }^{\circ} \mathrm{C}$ at the rate of $-0.3{ }^{\circ} \mathrm{C} / \mathrm{min}$, followed by plunging to liquid nitrogen. Vials were then stored in liquid phase of liquid nitrogen until thawing.

Table 2.1 Freezing programme for pancreatic islets

\begin{tabular}{llll}
\hline Step & Rate & Duration & Notes \\
\hline$+4{ }^{\circ} \mathrm{C}$ to $-7.4{ }^{\circ} \mathrm{C}$ & $-2{ }^{\circ} \mathrm{C} / \mathrm{min}$ & $5.75 \mathrm{~min}$ & \\
$-7.4{ }^{\circ} \mathrm{C}$ & $0{ }^{\circ} \mathrm{C}$ & $15 \mathrm{~min}$ & Hold temperature for nucleation \\
$-7.4{ }^{\circ} \mathrm{C}$ to $-40{ }^{\circ} \mathrm{C}$ & $-0.3{ }^{\circ} \mathrm{C} / \mathrm{min}$ & $108 \mathrm{~min}$ & Slow freezing \\
$-40{ }^{\circ} \mathrm{C}$ to $-196{ }^{\circ} \mathrm{C}$ & $>-1000{ }^{\circ} \mathrm{C} / \mathrm{min}$ & $10 \mathrm{~s}$ & Plunge to liquid nitrogen \\
\hline
\end{tabular}




\subsubsection{Thawing of pancreatic islets}

The thawing procedure for pancreatic islets was optimised during a series of experiments for human and mouse pancreatic islets (see Section 5.3.3), the protocols described here are the final optimised protocols.

Cryopreserved mouse pancreatic islet samples were removed from liquid nitrogen and transferred to water bath on dry ice. Rapid thawing in a $37{ }^{\circ} \mathrm{C}$ water bath was performed until the last ice crystal was visible in the vial and dilution of the cryoprotectant with culture medium (RMPI + $10 \%$ FCS) was performed over 24 min as described in Table 2.2. The final concentration of cryoprotectant was decreased to $10 \%$ of the original one (i.e. final concentration of DMSO in samples cryopreserved in $2 \mathrm{M}$ DMSO after performing this protocol was $0.2 \mathrm{M}$ ). Islets were then handpicked into a fresh Petri dish containing culture medium and either used immediately or cultured at $37{ }^{\circ} \mathrm{C}$ in $5 \% \mathrm{CO}_{2}$ humidified atmosphere.

Table 2.2 Cryoprotectant removal protocol for mouse pancreatic islets

\begin{tabular}{ccc}
\hline $\begin{array}{c}\text { Time after thawing } \\
(\mathrm{min})\end{array}$ & $\begin{array}{c}\text { Addition of CM } \\
(\mu \mathrm{l})\end{array}$ & $\begin{array}{c}\text { Concentration of CPA } \\
(\% \text { original concentration })\end{array}$ \\
\hline- & - & $\mathbf{1 0 0 . 0}$ \\
0 & 100 & $\mathbf{8 0 . 0}$ \\
2 & $"$ & 66.7 \\
4 & $"$ & 57.1 \\
6 & $"$ & 50.0 \\
8 & 200 & $\mathbf{4 0 . 0}$ \\
10 & $"$ & 33.3 \\
12 & $"$ & 28.6 \\
14 & $"$ & 25.0 \\
16 & 400 & $\mathbf{2 0 . 0}$ \\
18 & $"$ & 16.7 \\
20 & $"$ & 14.3 \\
22 & $"$ & 12.5 \\
24 & 800 & $\mathbf{1 0 . 0}$ \\
\hline
\end{tabular}

Notes: $\mathrm{CM}=$ culture medium; $\mathrm{CPA}=$ cryoprotectant; " = same as above

Human pancreatic islets were transferred from liquid nitrogen onto dry ice and transported to the $37{ }^{\circ} \mathrm{C}$ water bath where they were rapidly thawed until last crystal was visible. Cryovials were then centrifuged ( $300 \mathrm{~g}$ for $1 \mathrm{~min}$ ) and supernatant substituted for $1 \mathrm{~mL}$ of $0.75 \mathrm{M}$ sucrose solution in CMRL $+10 \%$ FCS. After 30 min incubation on ice, contents of cryovials were transferred to Petri dishes at room temperature and culture medium (CMRL $+10 \%$ FCS, Corning) was added every five minutes as outlined in Table 2.3 until the final 
concentration of cryoprotectants reached $2.2 \%$ of the original concentration (i.e. $0.04 \mathrm{M}$ DMSO if cryopreserved with $10 \%$ DMSO) and $0.15 \mathrm{M}$ sucrose. Islets were then handpicked into a fresh Petri dish containing culture medium and either used immediately or cultured at $37{ }^{\circ} \mathrm{C} 5 \% \mathrm{CO}_{2}$ humidified atmosphere.

Table 2.3 Cryoprotectant removal protocol for human pancreatic islets

\begin{tabular}{cccc}
\hline $\begin{array}{c}\text { Time after } \\
\text { thawing }+ \text { spin } \\
(\mathrm{min})\end{array}$ & $\begin{array}{c}\text { Addition of } \\
\text { CM/SUC } \\
(\mathrm{mL})\end{array}$ & $\begin{array}{c}\text { Concentration } \\
\text { of CPA }\end{array}$ & $\begin{array}{c}\text { Concentration } \\
\text { of SUC }\end{array}$ \\
\hline- & - & 100.0 & 0.0 \\
0 & $1(\mathrm{SUC})$ & 10.0 & 100.0 \\
30 & $1(\mathrm{CM})$ & 5.2 & 50.0 \\
35 & $1(\mathrm{CM})$ & 3.5 & 33.3 \\
40 & $2(\mathrm{CM})$ & 2.2 & 20.0 \\
\hline
\end{tabular}

Notes: $\mathrm{CM}=$ culture medium; $\mathrm{CPA}=$ cryoprotectant; $\mathrm{SUC}=0.75 \mathrm{M}$ sucrose

\subsubsection{Liquid nitrogen storage}

All cryopreserved samples were stored in liquid phase of liquid nitrogen inside one of three dewars available. The level of liquid nitrogen in these dewars was manually monitored twice a week and filled up as necessary. Sample database described in Section 2.1.4 was consulted prior to any deposit or removal of samples. Transferring of samples into and from the liquid nitrogen dewar was done in a rapid fashion and sample boxes were strictly handled on dry ice to decrease the impact of temperature fluctuations.

\subsection{Assays for single cell analysis}

\subsubsection{Cell counting}

Absolute cell number of fresh or thawed mononuclear cells was determined either manually using a haemocytometer (Improved Neubauer, Hawksley) or using Trucount ${ }^{\mathrm{TM}}$ tubes (BD Biosciences). To count with haemocytometer, the cell suspension was diluted with white blood cell counting fluid ( $2 \%$ acetic acid supplemented with crystal violet) or Trypan Blue solution (MP Biomedicals). Count of cells in four large squares ( $1 \mathrm{~mm}^{2}$ area each) was performed for each sample and average value was then used for the calculation of absolute cell count following this formula: 
Absolute cell count $=$ Average count per square $\times$ dilution factor $\times 10^{4} \times$ volume in $\mathrm{mL}$

To determine absolute cell count with Trucount ${ }^{\mathrm{TM}}$ tubes, known volume of cell suspension was pipetted into the Trucount tube and diluted with staining buffer (PBS $+0.1 \%$ bovine serum albumin $+0.1 \%$ sodium azide). Using BD FACSCanto ${ }^{\mathrm{TM}}$ II flow cytometer (BD Biosciences), at least 20,000 events were recorded. Cells were gated based on Syto13 and 7-AAD (7- Aminoactinomycin D) staining and beads were gated using the PE-Cy7 versus APC-Cy7 dot plot, found in upper right quadrant. Absolute cell count was calculated as:

$$
\text { Absolute cell count }=\frac{\text { cells in gate }}{\text { beads in gate }} \times \frac{\text { beads in Trucount tube }}{\mu L \text { of sample in Trucount tube }} \times \text { total volume of sample in } \mu L
$$

\subsubsection{Toxicity assays for mononuclear cells}

Fresh human splenocytes resuspended in DMEM $+10 \%$ FCS were aliquoted into wells of 96-well plates and incubated with $0-1000 \mu \mathrm{g} / \mathrm{ml}$ of PP-50/PP-75 or $0-800 \mathrm{mM}$ trehalose at $37{ }^{\circ} \mathrm{C}$ with $5 \% \mathrm{CO}_{2}$ for 2,24 or $48 \mathrm{~h}$. Cells were washed off after the incubation period with PBS and viability of the cells was analysed immediately with Syto13 (Invitrogen, 1:500,000 dilution) and 7-AAD Viability Staining Solution (eBioscience, 1:20 dilution). Absolute cell count was determined using BD Trucount ${ }^{\mathrm{TM}}$ tubes (BD Biosciences).

\subsubsection{Culture assays for mononuclear cells}

Fresh human splenocytes were resuspended in $200 \mathrm{mM}$ trehalose (in DMEM + $10 \%$ FCS) and $500 \mu \mathrm{L}$ were aliquoted into $1.5 \mathrm{~mL}$ Eppendorf tubes (one for each condition). Each tube was then incubated at a different temperature $\left(3{ }^{\circ} \mathrm{C}\right.$ in the fridge, $20{ }^{\circ} \mathrm{C}$ at room temperature and $37^{\circ} \mathrm{C}$ in the water bath). At each time point measured, the vial was well mixed and small known volume of the suspension was taken out into a Trucount tube. The cells were then stained with Syto13 (Invitrogen, 1:500,000 dilution) and 7-AAD (eBioscience, 1:20 dilution) using no-wash procedure and data collected on a flow cytometer. Absolute counts of alive nucleated cells were normalised to absolute counts of alive nucleated cells at time 0 .

\subsubsection{Flow cytometry of mononuclear cells}

Human cells (fresh or thawed) were resuspended in staining buffer (PBS $+0.1 \%$ bovine serum albumin $+0.1 \%$ sodium azide) and treated with human or mouse FcR blocking agent (Miltenyi Biotech, 1:20 dilution) for $15 \mathrm{~min}$ at $4{ }^{\circ} \mathrm{C}$ prior to staining. After one washing step 
Table 2.4 Antibodies and stains used for the analysis of fresh and thawed mononuclear cells

\begin{tabular}{llll}
\hline Antibody/stain & Conjugate & Supplier & Dilution used \\
\hline 7-AAD & - & eBioscience & $1: 20$ \\
Annexin V & APC & Thermo Fisher Scientific & $1: 200$ \\
CD19 & APC-Vio770 & Miltenyi Biotech & $1: 20$ \\
CD19 & PE & BioLegend & $1: 200$ \\
CD19 & PE-Vio770 & Miltenyi Biotech & $1: 20$ \\
CD3 & PE-Cy7 & BioLegend & $1: 400$ \\
CD34 & PE & eBioscience & $1: 20$ \\
eFluor 780 & - & eBioscience & $1: 2000$ \\
Human haematopoietic & APC & eBioscience & $1: 20$ \\
lineage cocktail & & & \\
Syto13 & - & Thermo Fisher Scientific & $1: 500,000$ \\
\hline
\end{tabular}

Notes: For the antibodies, fluorochrome conjugates are indicated. Suppliers and dilutions used (determined by titration for each of the reagents) are stated for all reagents

(centrifugation at $200 \mathrm{~g}$ for $4 \mathrm{~min}$ ), cells were stained by fluorochrome conjugated antibodies (each was titrated to ensure the correct concentration, see Table 2.4) in staining buffer for $15 \mathrm{~min}$ at $4{ }^{\circ} \mathrm{C}$ protected from light. The viability stain used was either 7-AAD or eFluor 780, for the latter the staining was done subsequently in PBS for 20 min at $4{ }^{\circ} \mathrm{C}$, protected from light. Staining for annexin V was done in annexin binding buffer (50 mM HEPES, $700 \mathrm{mM}$ $\mathrm{NaCl}, 12.5 \mathrm{mM} \mathrm{CaCl}_{2}, \mathrm{pH}=7.4$ ) for $15 \mathrm{~min}$ in dark at room temperature. Information about the antibodies and stains used for mononuclear cells is summarised in Table 2.4. The choice of antibody panels used for the experiments is justified in Appendix B.

The analysis of the stained samples was done using a 6-colour flow cytometer BD FACSCanto $^{\mathrm{TM}}$ II and computer equipped with the BD FACSDiva ${ }^{\mathrm{TM}}$ software (BD Biosciences, version 6.1.3). For each analysis, unstained control and single stained compensation controls were included and automatic compensation was performed. After recording data from the samples, FCS files were exported and further analysed by the FlowJo software (version 10.1). The gating strategy for determining the viability of cells and enumerating the haematopoietic progenitors is shown in Appendix B.

\subsubsection{Fluorescence activated cell sorting (FACS)}

Thawed human mononuclear cells were counted and stained with fluorochrome-conjugated antibodies (Table 2.5) for $30 \mathrm{~min}$ at $4{ }^{\circ} \mathrm{C}$. Single stained compensation beads (BD CompBeads, BD Biosciences) were used as compensation controls, except for the viability stain (Zombie 
Aqua $^{\mathrm{TM}}$, Biolegend) where cells were used for compensation setup. FACS was performed using BD FACSAria ${ }^{\mathrm{TM}}$ Fusion or BD FACSAria ${ }^{\mathrm{TM}}$ III cell sorter (provided by NIHR Cambridge BRC Cell Phenotyping Hub) and the desired number of cells of target population from each sample was collected into a $1.5 \mathrm{~mL}$ Eppendorf tube. Cells were kept on ice during the whole procedure.

The target population collected for the colony forming units assay was Zombie Aqua ${ }^{-}$,

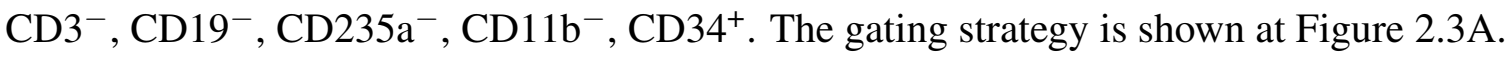

Table 2.5 Antibodies and stains used for the fluorescence cell sorting of mononuclear cells

\begin{tabular}{llll}
\hline Antibody/stain & Conjugate & Supplier & Dilution used \\
\hline CD11b & APC-Cy7 & BioLegend & $1: 300$ \\
CD19 & Alexa700 & BioLegend & $1: 300$ \\
CD235a & PE & BD Biosciences & $1: 1000$ \\
CD3 & FITC & BD Biosciences & $1: 500$ \\
CD34 & APC & BD Biosciences & $1: 200$ \\
CD38 & PE-Cy7 & BioLegend & $1: 100$ \\
Zombie Aqua & - & BioLegend & $1: 2000$ \\
\hline
\end{tabular}

Notes: For the antibodies, fluorochrome conjugates are indicated. Suppliers and dilutions used (determined by titration for each of the reagents) are stated for all reagents

\subsubsection{Haematopoietic colony forming unit (CFU) assay}

Aliquots of $2.5 \mathrm{~mL}$ MethoCult ${ }^{\mathrm{TM}}$ medium (H84435, StemCell Technologies) were thawed at $4{ }^{\circ} \mathrm{C}$. Eppendorf tubes containing CD $34^{+}$cells from bone marrow after the cell sorting were centrifuged at $500 \mathrm{~g}$ for $5 \mathrm{~min}$ and supernatant aspirated to leave approximately $50 \mu \mathrm{L}$ of medium in the tube. The cells were resuspended and the whole volume was then transferred into a tube of MethoCult ${ }^{\mathrm{TM}}$. The suspension was mixed by aspirating up and down several time using $1 \mathrm{~mL}$ syringe with $18 \mathrm{G}$ needle. Tubes were allowed to stand for $15 \mathrm{~min}$ to let any bubbles rise to the top. Using the syringe and needle, the suspension was drawn from a Methocult ${ }^{\mathrm{TM}}$ tube and plated in duplicates into the wells of a 6-well plate (SmartDish ${ }^{\mathrm{TM}}$, Stemcell Technologies), $1 \mathrm{~mL}$ into each well (Figure 2.3B). The 6-well plates were then placed into a larger container together with two $35 \mathrm{~mm}$ Petri dishes containing sterile PBS to keep the culture hydrated and incubated at $37{ }^{\circ} \mathrm{C}$ and $5 \% \mathrm{CO}_{2}$ for 14 days.

On days 11 and 14, the colonies of haematopoietic cells were counted by STEMvision ${ }^{\mathrm{TM}}$ (Stemcell Technologies) automated colony counter. Images of the colonies were acquired using the STEMvision ${ }^{\mathrm{TM}}$ Acquisition application (Figure 2.3D). The automatic count of 
three colony types (Figure 2.3C) by STEMVision ${ }^{\mathrm{TM}}$ Analyser application was then manually validated and adjusted using the STEMvision ${ }^{\mathrm{TM}}$ Marker software (Figure 2.3E). The colony counts from all conditions were normalised to the number of $\mathrm{CD} 34^{+}$cells seeded.

After the colonies were counted on day 14, cells from the dishes were washed with PBS + $3 \%$ FCS, the suspension was harvested, combining the duplicate cultures, and washed twice to remove the Methocult ${ }^{\mathrm{TM}}$ medium. Flow cytometry was then performed, using the markers for erythrocytes (Glycophorin A/CD235a), myeloid cells (CD33), monocytes/macrophages (CD14) and granulocytes (CD15) (Table 2.6). Gating strategy is shown at Figure 2.3F. The stained samples were transferred into BD Trucount ${ }^{\mathrm{TM}}$ tubes (BD Biosciences) to determine the absolute count of the harvested cells.

Table 2.6 Antibodies and stains used for the analysis of cells harvested from the colonies of haematopoietic cells grown for the CFU assay

\begin{tabular}{llll}
\hline Antibody/stain & Conjugate & Supplier & Dilution used \\
\hline 7-AAD & - & eBioscience & $1: 20$ \\
CD235a & FITC & Miltenyi Biotech & $1: 20$ \\
CD33 & PE & Miltenyi Biotech & $1: 50$ \\
CD14 & PE-Vio770 & Miltenyi Biotech & $1: 100$ \\
CD15 & APC & Miltenyi Biotech & $1: 20$ \\
\hline
\end{tabular}

Notes: For the antibodies, fluorochrome conjugates are indicated. Suppliers and dilutions used (determined by titration for each of the reagents) are stated for all reagents

\subsubsection{Total protein assay}

Fresh mononuclear cells were incubated in 96-well plate $\left(10^{6}\right.$ cells per well) with a range of polymer concentrations for $4 \mathrm{~h}$ at room temperature. After the incubation, cells were centrifuged (at $300 \mathrm{~g}$ for $5 \mathrm{~min}$ ) and resuspended in NP-40 cell lysis buffer (Invitrogen) supplemented with cOmplete ${ }^{\mathrm{TM}}$ Protease inhibitor (Roche). Lysates were diluted and $25 \mu \mathrm{L}$ was transferred into a new flat-bottomed 96-well plate for the assay. Same volume of prediluted bovine serum albumin (BSA) standards were also transferred into separate wells of the new plate. Total protein measurement was performed using the BCA Protein Assay Kit II (BioVision) according to manufacturer's instructions. Briefly, $200 \mu \mathrm{L}$ of prepared detection mix was added to each well with standard or sample and the plate was incubated at $37{ }^{\circ} \mathrm{C}$ for 30 minutes. Measurement was then performed using the FLUOstar Optima plate reader (BMG Labtech), excitation $480 \mathrm{~nm}$, emmision $560 \mathrm{nM}$. 

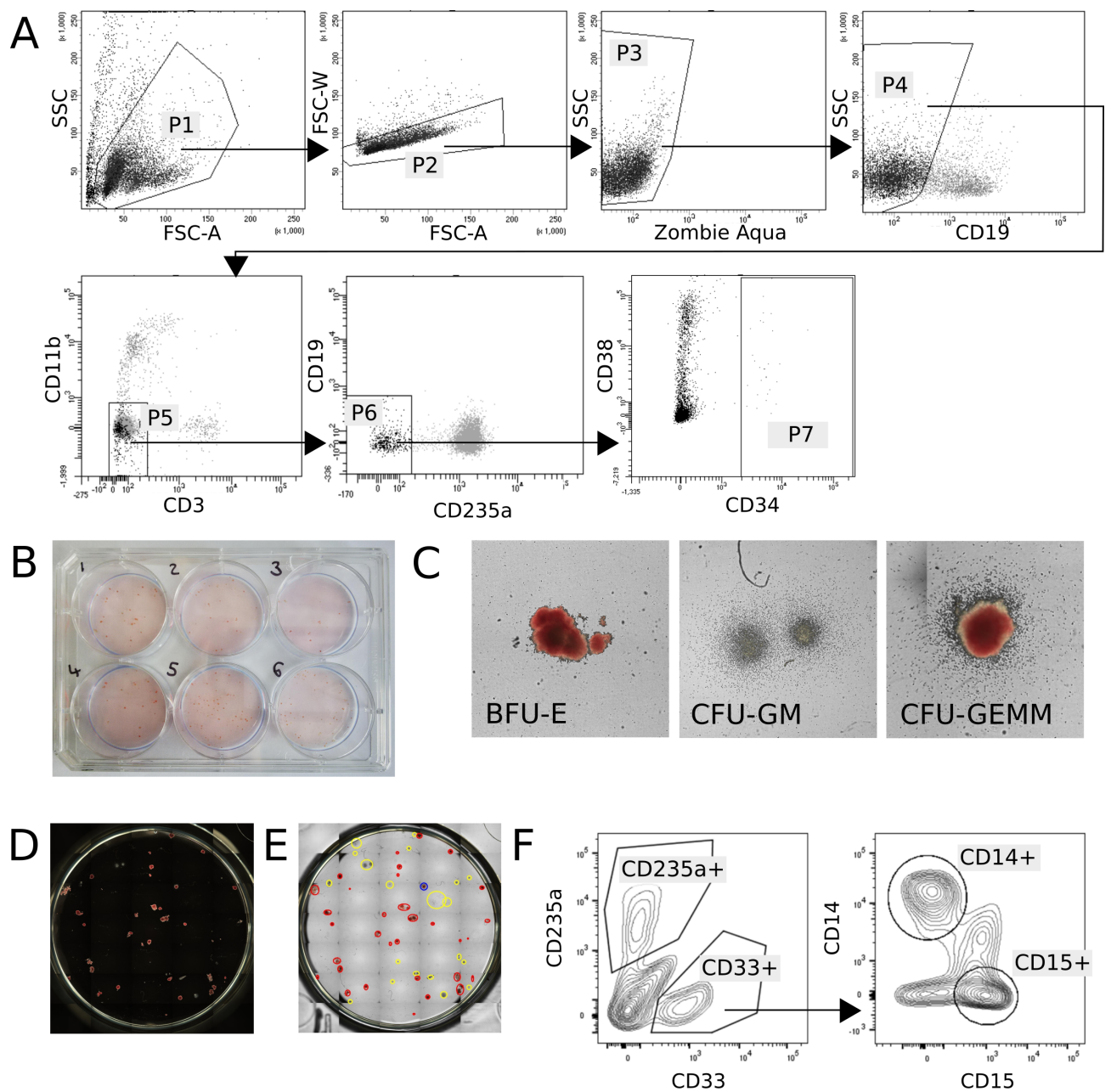

Fig. 2.3 Colony forming units assay. (A) Gating strategy for fluorescence activate cell sorting. Gate $\mathrm{P} 1=$ cell population, $\mathrm{P} 2=$ singlets, $\mathrm{P} 3=$ Zombie Aqua $^{-}$(viable cells), $\mathrm{P} 4=\mathrm{CD} 19^{-}$cells, $\mathrm{P} 5=\mathrm{CD}^{-}$ $\mathrm{CD} 11 \mathrm{~b}^{-}$cells, $\mathrm{P} 6=\mathrm{CD} 19^{-} \mathrm{CD} 235 \mathrm{a}^{-}$cells, $\mathrm{P} 7=\mathrm{CD} 34^{+}$cells. Target population for sorting is Zombie Aqua $^{-}, \mathrm{CD}^{-}, \mathrm{CD} 19^{-}, \mathrm{CD} 235 \mathrm{a}^{-}, \mathrm{CD} 11 \mathrm{~b}^{-}, \mathrm{CD}^{+} 4^{+}$. (B) SmartDish ${ }^{\mathrm{TM}}$ with duplicate cultures seeded with $\mathrm{CD} 34^{+}$cells; after 14 days of culture red and white haematopoietic colonies are visible. (C) Haematopoietic colonies were classified into three categories: red BFU-E (burst-forming unit erythroid), white CFU-GM (granulocyte-macrophage colony forming unit) and mixed CFUGEMM (granulocyte-erythrocyte-monocyte-megakaryocyte colony forming unit). (D) Reconstructed dark-field image of one SmartDish ${ }^{\mathrm{TM}}$ well with haematopoietic colonies after 14 days of culture photographed by STEMVision $^{\mathrm{TM}}$. (E) Automated recognition of colonies by the STEMVision ${ }^{\mathrm{TM}}$ Analyser software. Different colony types are marked by different colour rings: red for BFU-E, yellow for CFU-GM and blue for CFU-GEMM. (F) Gating strategy for analysis of cells from colonies after 14 days of culture. First of all, cells, singlets and alive cells were selected by gating (as shown in (A)). Myeloid and erythroid cells were then distinguished based on their expression of CD235a and $\mathrm{CD} 33$. $\mathrm{CD} 33^{+}$myeloid cells were further distinguished as $\mathrm{CD} 14^{+}$monocytes/macrophages and $\mathrm{CD} 15^{+}$granulocytes. Results were then expressed as percentage of erythroid cells, myeloid cells, macrophages or granuloces from the total cell count. 

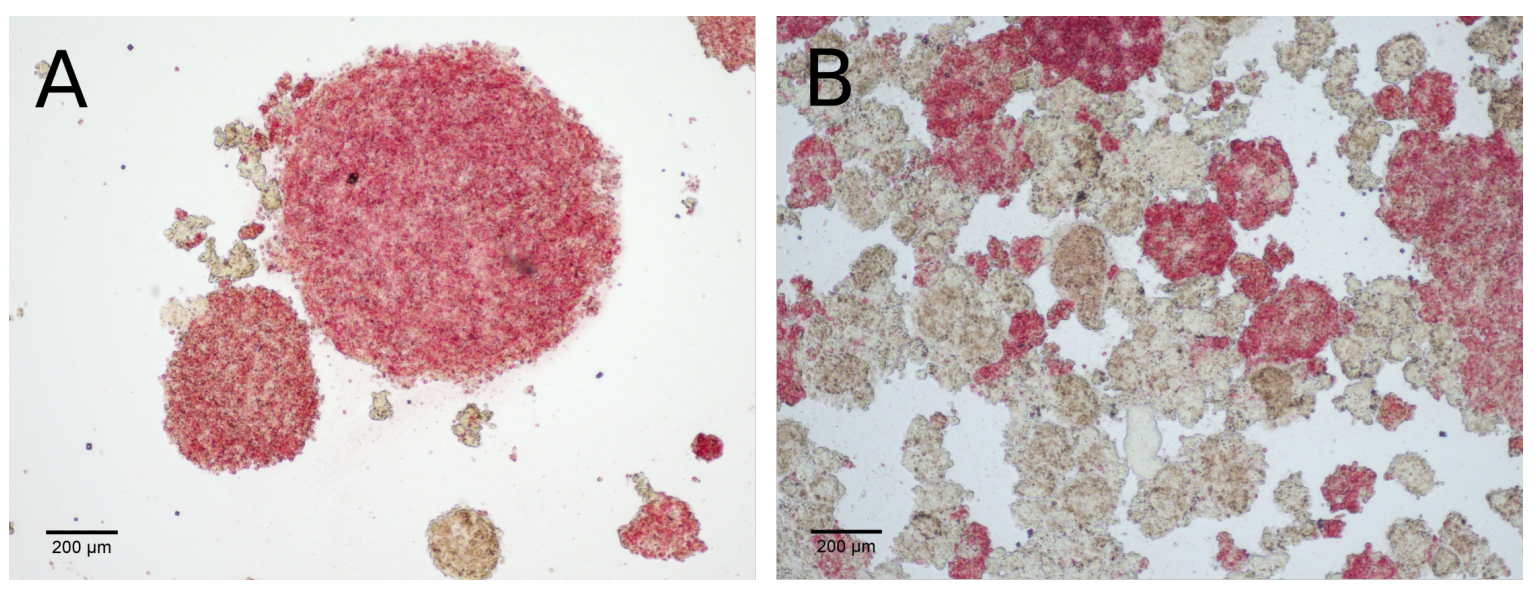

Fig. 2.4 A fresh sample of human pancreatic islets stained by dithizone dye. (A) Example of very pure batch of islets containing almost solely islets (red colour) and no acinar tissue (grey colour). Human islet size is typically very variable and this image shows a particularly large islet. (B) Example of pancreatic islet sample containing large percentage of acinar tissue. Both images were taken at $4 x$ objective magnification.

\subsection{Assays for pancreatic islets}

\subsubsection{Assessment of purity by dithizone staining}

On arrival of fresh human pancreatic islets, the purity of the batch was validated by dithizone staining. The stock of dithizone was prepared at the concentration of $0.2 \mathrm{mg} / \mathrm{mL}$ and $\mathrm{pH} 7.4$ and aliquots were stored at $-20^{\circ} \mathrm{C}$. Thawed aliquot of dithizone was filtered through $0.22 \mu \mathrm{m}$ syringe filter before staining. An aliquot of approximately 200 IEQ was obtained from the islet suspension stock and resuspended in $2 \mathrm{~mL}$ of the dithizone stain. After 10 - $12 \mathrm{~min}$ of incubation islets were immediately imaged on a glass slide with cover slip using bright field illumination on the Olympus IX81 inverted fluorescence microscope. The pancreatic islets stain red as the dye binds to the zinc ions in the secretory granules of $\beta$-cells. The acinar tissue is not stained by this dye (Figure 2.4). The purity of the islet sample was visually assessed as a percentage of red-staining tissue in the whole sample.

\subsubsection{Assessment of viability by fluorescent imaging}

Islets were handpicked and placed in $300 \mu \mathrm{L}$ of PBS. Fresh mastermix of 1:10 dilution of propidium iodide (PI) stock $(0.5 \mathrm{mg} / \mathrm{mL}$, Sigma-Aldrich) and 1:100 dilution of fluorescein diacetate (FDA) stock ( $5 \mathrm{mg} / \mathrm{mL}$, Sigma-Aldrich) were prepared and used for staining within $30 \mathrm{~min} .20 \mu \mathrm{L}$ of the mastermix was added to the islet sample and immediately imaged by an Olympus IX81 inverted fluorescence microscope (FDA using the FITC filter and PI using 


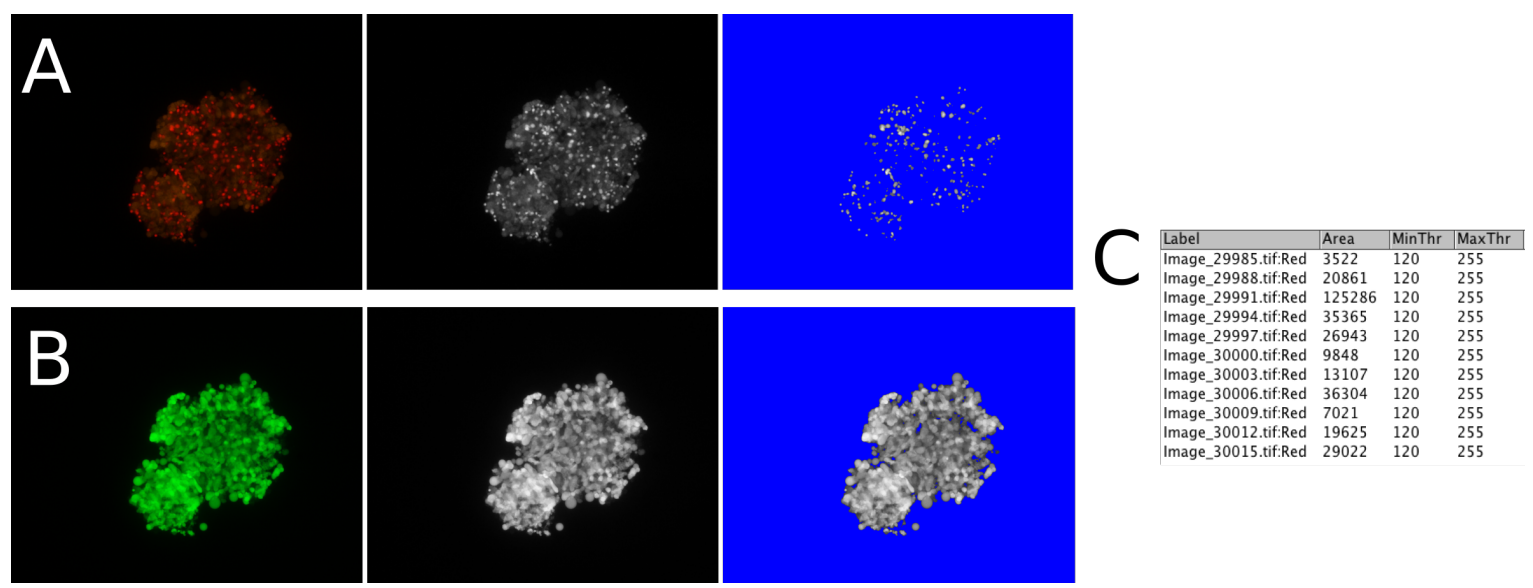

Fig. 2.5 Image analysis of islet viability staining. Example of analysis using a cryopreserved mouse islet stained with FDA and PI. (A) Images taken in TRITC channel were used for analysis of PI-stained cells within islet. First, the original image was converted to RGB stack, followed by applying the threshold to include only the positive areas while everything else is converted to a blue background. (B) Analogical analysis pipeline for the FITC channel containing FDA staining. (C) Table generated after applying the code above to a folder of images cryopreserved under a certain condition. The table contains the Image ID (Label), the positive area measurement (Area) and the lowed and upper threshold values (MinThr, MaxThr). All microscope images were taken at 10x objective magnification. $\mathrm{FDA}=$ fluorescein diacetate $\mathrm{PI}=$ propidium iodide .

the TRITC filter). Each channel was taken as a separate image and the parameters of the microscope (exposure time, gain) were kept the same throughout each experiment to enable unbiased quantification. The FDA positive (alive) and PI positive (dead) area was measured using ImageJ. Briefly, the images were converted to RGB stack and threshold was applied to select only the positive area in the appropriate colour channel (Figure 2.5A and B). The top threshold was always kept at the maximum (255) while the lower threshold value was determined by manually selecting ideal threshold value for three random images from each experiment. This threshold was then applied to all images from the experiment.

A simple macro code was written to perform the batch analysis and its annotated version is shown below for both FDA and PI images (FDA image analysis required an additional line to switch to the appropriate colour channel).

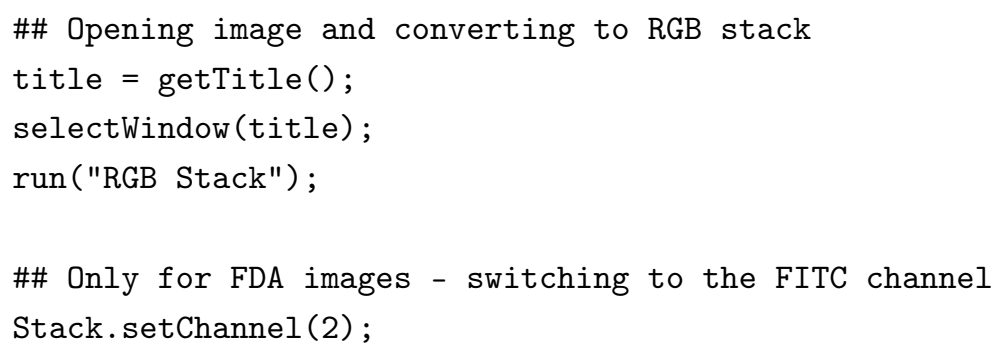




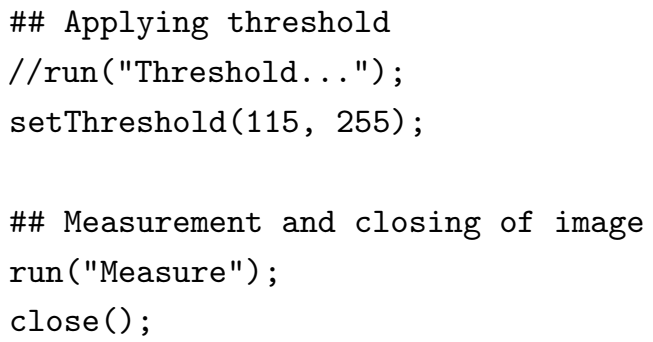

Using this code, a table was generated which contained the positive area measurement for each image (Figure 2.5C). These values were then transferred to Microsoft Excel. Viability of each imaged islet was calculated using following formula:

$$
\text { Percentage islet viability }=\frac{\text { FDA positive area }}{F D A \text { positive area }+ \text { PI positive area }} \times 100 \text {. }
$$

\subsubsection{Assessment of viability by flow cytometry}

Sufficient amounts of pancreatic islets (approximately 2000 IEQ produces around $10^{6}$ cells) were trypsinised by $5 \mathrm{~min}$ incubation in $0.05 \%$ trypsin $0.53 \mathrm{mM}$ EDTA (Sigma-Aldrich). Trypsinised islets were then pipetted several times through p1000 and p200 pipette tips for full disintegration. Trypsin reaction was then stopped by addition of culture medium containing FCS. Cells were counted using Trucount ${ }^{\mathrm{TM}}$ tubes (BD Biosciences) and stained for viability with combination of FDA (alive cells) and PI (dead cells). The cells were incubated with the stains (with the exception of PI) for $25 \mathrm{~min}$ at $37{ }^{\circ} \mathrm{C}$ and washed twice in staining buffer before analysis. Samples and appropriate controls were run on FACSCanto ${ }^{\mathrm{TM}}$ II flow cytometer equipped with BD FACSDiva ${ }^{\mathrm{TM}}$ software (BD Biosciences, version 6.1.3). Subsequent analysis was done in FlowJo software (version 10.1).

\subsubsection{Diffusion assays for pancreatic islets}

Fresh mouse pancreatic islets were pre-stained with $400 \mu \mathrm{g} / \mathrm{mL}$ Hoechst 33342 nucleic acid stain (ThermoFisher) under different conditions (variations in temperature, DMSO concentration and $g$ force). At the end of the incubation time, islets were washed with PBS and handpicked with minimal volume of medium $(\leq 10 \mu \mathrm{L})$ into a Peel-A-Way embedding conical mold (Polysciences, Inc.). $60-80 \mu \mathrm{L}$ of Optimal Cutting Temperature Compound (OCT) was carefully mixed with the handpicked islets in the bottom of the mold to avoid any bubbles and the drop was frozen on dry ice. Once rigid, it was covered with more OCT to create a block which is easy to cryosection, again frozen on dry ice and stored at $-80{ }^{\circ} \mathrm{C}$ until sectioning. 


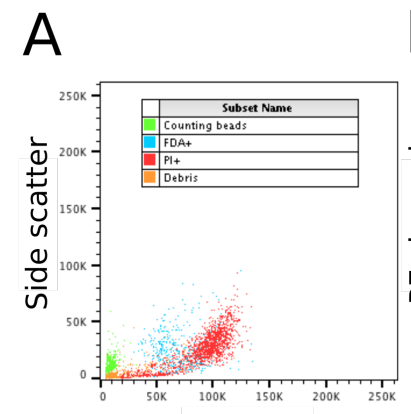

Forward scatter

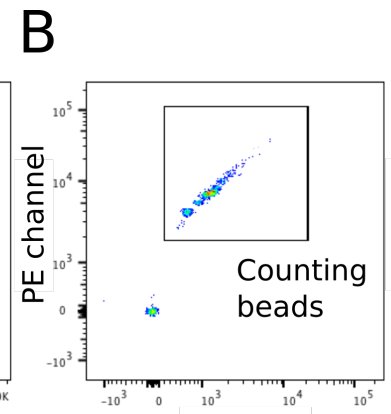

APC channel

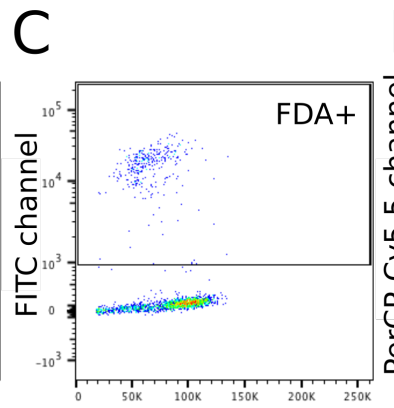

Forward scatter

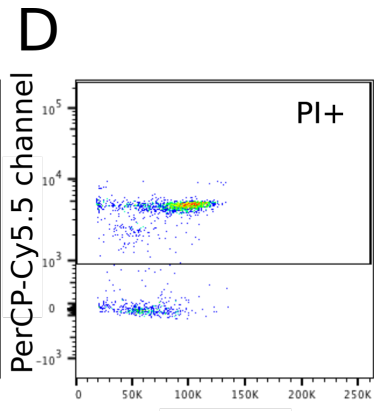

Forward scatter

Fig. 2.6 Gating strategy for pancreatic islet flow cytometry. (A) FSC/SSC view of the recorded cytometry events (singlets only). Counting beads shown in green, $\mathrm{FDA}^{+}$(live) cells in blue and $\mathrm{PI}^{+}$ (dead) events in red. (B) Gating on counting beads which can be done in multiple combinations of different fluorescent channels. Channels unused by any fluorochromes were used for this analysis. (C) Gating on FDA ${ }^{+}$events in the FSC/FITC channel view. (D) Gating on $\mathrm{PI}^{+}$events in the FSC/PerCPCy5.5 channel view. FDA = fluorescein diacetate; FCS $=$ forward scatter; PI = propidium iodide; $\mathrm{SSC}=$ side scatter.

Cryosectioning of temperature-equilibrated OCT blocks was done using Hacker Bright OTF5000 cryostat (Hacker Instruments and Industries Inc.) at a thickness of $11 \mu \mathrm{m}$. Sections were transferred to a room temperature microscope slide and frozen immediately on dry ice to avoid dehydration. Prior to imaging, coverslips were mounted onto the slides using a dry mount method. Wet mounting was not suitable since it allowed movement of dye molecules across the samples. Therefore, dry mounting was used in which two thin strips $(2-3 \mathrm{~mm})$ of double-sided tape were stuck on sides of each glass coverslip (Figure 2.7A). Frozen slides were rapidly thawed, coverslip mounted on top using the tape and frozen again to avoid any dehydration. Slides were kept on dry ice and were only removed while being imaged.

Imaging was performed using the Leica Sp5 (Leica Microsystems) inverted confocal microscope (NIHR Cambridge BRC Cell Phenotyping Hub) as outlined in Section 2.7.1. Bright field and fluorescent images of 3 - 10 islet sections were taken for each experimental condition (Figure 2.7B). Only sufficiently round islets were included in the analysis as the image analysis method was not suitable to analyse irregularly shaped or elliptical islets. Image analysis was done in ImageJ plugin Concentric Circles as outlined in Figure 2.7C).

\subsubsection{Toxicity assays for pancreatic islets}

For each tested condition, 3 wells of 15 - 20 islets were handpicked into a 24 -well flatbottomed plate, covered with $500 \mu \mathrm{L}$ tested solution and incubated at $37{ }^{\circ} \mathrm{C}, 5 \% \mathrm{CO}_{2}$ humidified atmosphere. For every experiment, a culture medium control was included (RMPI $+10 \%$ FCS $1 \%$ Penicillin/Streptomycin for mouse islets or CMRL $+10 \%$ FCS $+1 \%$ 

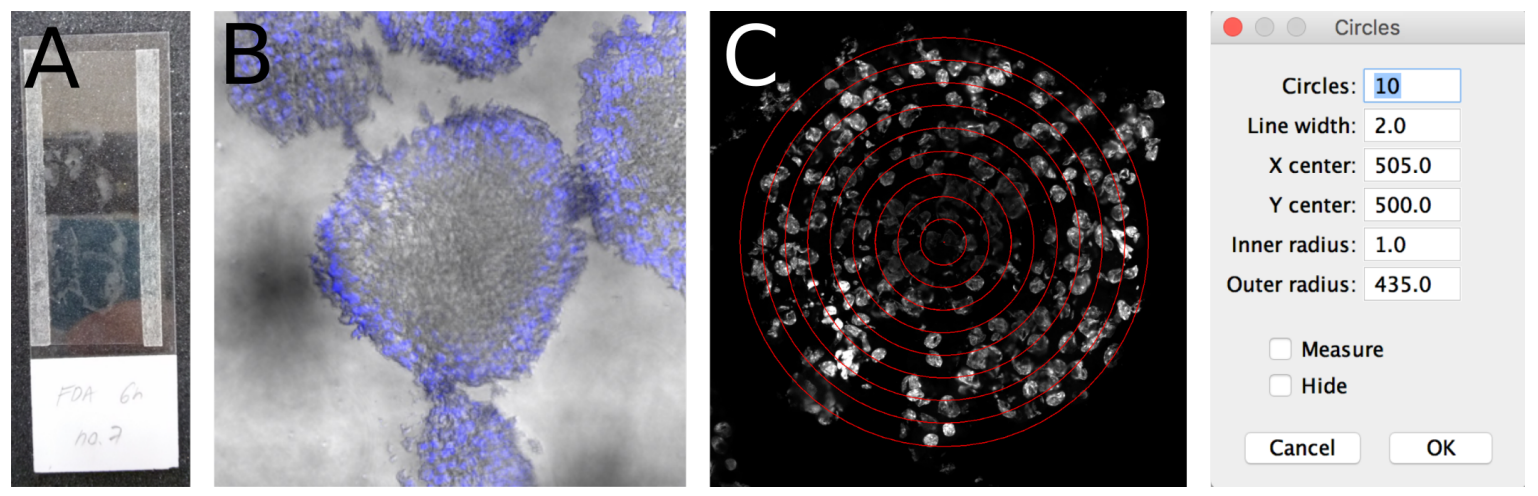

Fig. 2.7 Method used for assessing dye diffusion into the centre of pancreatic islets. (A) Dry mount method of mounting a coverslip using a double-sided tape. (B) Example of an image taken on a confocal microscope showing a section of an islet pre-stained with Hoechst 33342 nuclear dye. (C) Concentric circles plugin in ImageJ used for calculating the average fluorescent intensity along each concentric circle. Using these values, a staining profile of each islet can be generated. Multiple parameters can be changed to adjust for the size and position of the islet on the image. In real analysis, the circles were drawn 5 pixels apart ( $30-80$ circles per islet depending on the size of the islet).

Penicillin/Streptomycin for human islets). These three wells were then used to measure viability at three different time points ( $2 \mathrm{~h}, 24 \mathrm{~h}$ and $48 \mathrm{~h})$. At the end of each time point, the content of the respective wells (medium + islets) were transferred into a fresh tube for fluorescent viability staining (described in Section 2.6.2). Viability of islets at at beginning of experiment was measured and used as a $0 \mathrm{~h}$ control for all conditions.

\subsubsection{ATP/ADP assay}

Fifty pancreatic islets for each experimental sample (fresh or thawed) were handpicked into a $1.5 \mathrm{~mL}$ Eppendorf tube and adenonucleotides were extracted by adding $1 \mathrm{~mL}$ of ice-cold perchloric acid ( $3 \% \mathrm{v} / \mathrm{v} \mathrm{HClO}_{4}, 2 \mathrm{mM} \mathrm{Na} 2 \mathrm{EDTA}, 0.5 \%$ Triton X-100) followed by vortexing. Extracted samples were stored at $-80{ }^{\circ} \mathrm{C}$ until ATP and ADP quantification using luciferase-based assay described by Strehler [169].

Briefly, samples for ATP and ADP standard curves were prepared from $20 \mathrm{mM}$ stock solutions of ATP and ADP sodium salts (Sigma) serially diluted in the perchloric acid extractant. In the next step, $400 \mu \mathrm{L}$ of each experimental and standard curve sample was pH-neutralised using ice-cold $\mathrm{KOH}$ solution (2 M KOH, 2 mM Na 2 EDTA, $50 \mathrm{mM}$ MOPS) until white $\mathrm{KClO}_{4}$ precipitate formed. Samples were then centrifuged $\left(17000 \mathrm{~g}, 1 \mathrm{~min}, 4{ }^{\circ} \mathrm{C}\right)$ and supernatant used for further measurements.

$250 \mu \mathrm{L}$ of experimental sample supernatant was prepared for quantification of ADP by degradation of endogenous ATP (Figure 2.8A). This was done by incubating the supernatant 


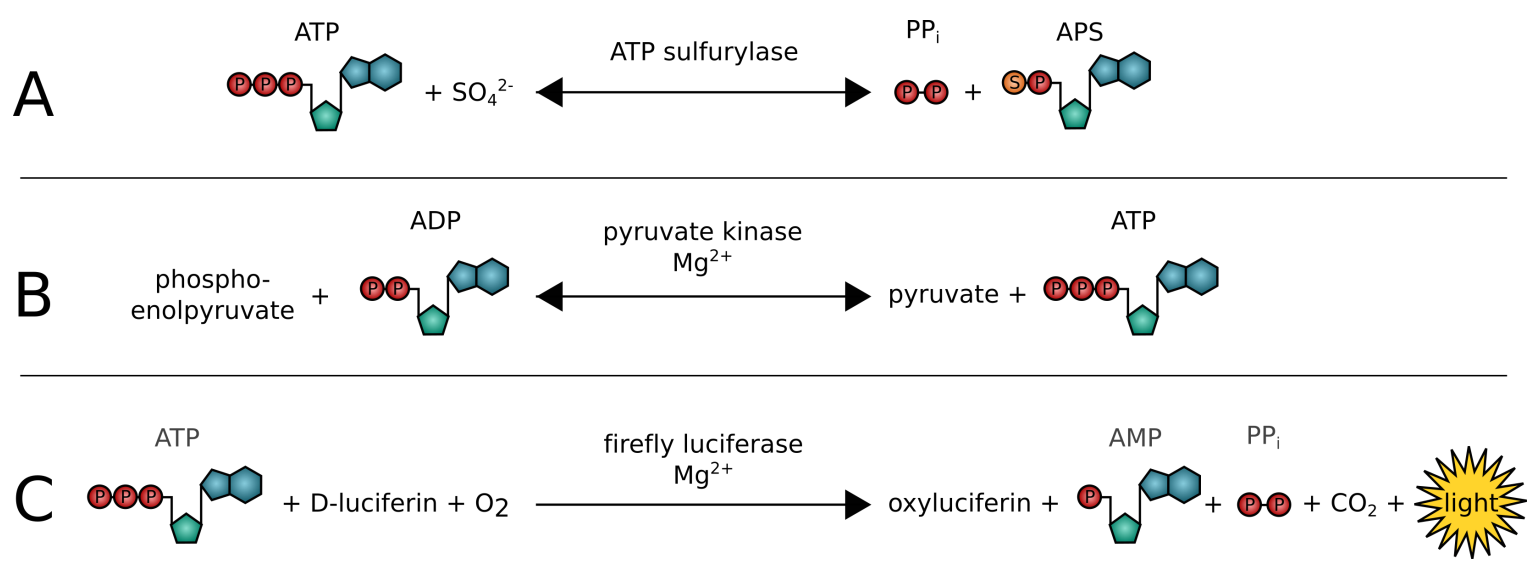

Fig. 2.8 ATP/ADP ratio assay reactions. (A) Degradation of endogenous ATP: ATP sulfurylase catalyses reaction of ATP and sulfate to form pyrophosphate and adenosine 5'-phosphosulfate (APS), which depletes ATP present in the sample. (B) Conversion of ADP to ATP: In the presence of phosphoenolpyruvate, pyruvate kinase converts ADP to ATP along with a pyruvate by-product. (C) Luciferase step: Firefly luciferase catalyses D-luciferin oxygenation accompanied by emission of light in the presence of ATP and oxygen. The amount of emitted light correlates with the amount of ATP in the sample (or ADP converted to ATP in (B)).

with $250 \mu \mathrm{L}$ ATP sulfurylase assay buffer $\left(20 \mathrm{mM} \mathrm{Na}_{2} \mathrm{MoO}_{4}, 5 \mathrm{mM}\right.$ GMP, $0.2 \mathrm{U}$ ATP sulfurylase (New England Biolabs), in Tris- $\mathrm{HCl}$ buffer (100 mM Tris- $\mathrm{HCl}, 10 \mathrm{mM} \mathrm{MgCl} 2$ (pH 8.0))) for $30 \mathrm{~min}$ at $30{ }^{\circ} \mathrm{C}$ while shaking, followed by heating to $100{ }^{\circ} \mathrm{C}$ for $5 \mathrm{~min}$ and cooling on ice.

$100 \mu \mathrm{L}$ of standards, one $100 \mu \mathrm{L}$ sample for ATP measurement and two $200 \mu \mathrm{L}$ samples for ADP measurement were mixed in luminometer tubes with $400 \mu \mathrm{L}$ Tris-acetate buffer (100 $\mathrm{mM}$ Tris, $2 \mathrm{mM} \mathrm{Na} 2 \mathrm{EDTA}, 50 \mathrm{mM} \mathrm{MgCl} 2$, $\mathrm{pH}$ adjusted to 7.75 with glacial acetic acid). To one of the ADP measurement samples, $10 \mu \mathrm{L}$ pyruvate kinase solution (100 mM PEP, $6 \mathrm{U}$ pyruvate kinase suspension (Sigma)) was added and incubated for $30 \mathrm{~min}$ at $25^{\circ} \mathrm{C}$ in the dark to convert ADP to ATP (Figure 2.8B). The other ADP measurement sample remained unconverted and served as a blank.

For all standard curve and experimental samples, $100 \mu \mathrm{L}$ Luciferase/Luciferin Solution (7.5 mM DTT, $0.4 \mathrm{mg} / \mathrm{ml} \mathrm{BSA,} 1.92 \mu \mathrm{g}$ luciferase/ml (Sigma), $120 \mu \mathrm{M}$ D-luciferin (Sigma), in Tris-acetate buffer ( $25 \%$ v/v glycerol)) was auto-injected and luciferase luminescence measured using Berthold AutoLumat Plus luminometer three times for 1 min (Figure 2.8C).

\subsubsection{Static glucose stimulation assay}

Salts for Krebs-Ringer Bicarbonate buffer (KRB, composition in Table 2.7) were dissolved in $\mathrm{ddH}_{2} \mathrm{O}$, filter-sterilised and stored at room temperature for a maximum of one month. On 
the day of the experiment, working KRB solutions were prepared by adding $10 \%$ FCS and 1.65 M glucose stock solution to reach $3.3 \mathrm{mM}$ (low glucose $\mathrm{KRB}$ ) or $16.7 \mathrm{mM}$ (high glucose $\mathrm{KRB}$ ) glucose concentration. Sufficient amount of $3.3 \mathrm{mM}$ and $16.7 \mathrm{mM} \mathrm{KRB}$ were warmed up to $37^{\circ} \mathrm{C}$.

Table 2.7 Composition of Krebs-Ringer Bicarbonate Buffer

\begin{tabular}{lc}
\hline Chemical & Concentration $(\mathrm{mM})$ \\
\hline $\mathrm{NaCl}$ & 137 \\
$\mathrm{KCl}$ & 4.7 \\
$\mathrm{KH}_{2} \mathrm{PO}_{4}$ & 1.2 \\
$\mathrm{MgSO}_{4} \cdot 7 \mathrm{H}_{2} \mathrm{O}$ & 1.2 \\
$\mathrm{CaCl}_{2} \cdot 2 \mathrm{H}_{2} \mathrm{O}$ & 2.5 \\
$\mathrm{NaHCO}_{3}$ & 25 \\
\hline
\end{tabular}

Fresh or thawed pancreatic islets were thoroughly washed from any leftover cryoprotectant (to avoid an effect of remaining trehalose on the insulin secretion). The islets were handpicked into a Petri dish containing $5 \mathrm{~mL}$ pre-warmed $3.3 \mathrm{mM} \mathrm{KRB}$ and incubated for $30 \mathrm{~min}$ at $37^{\circ} \mathrm{C}, 5 \% \mathrm{CO}_{2}$. For each tested condition, either 70 (parallel set-up, Figure 2.9A) or 40 (serial set-up, Figure 2.9B) islets were handpicked. After $30 \mathrm{~min}$, all islets were handpicked into a fresh Petri dish with $5 \mathrm{~mL}$ or pre-warmed $3.3 \mathrm{mM}$ KRB to wash any insulin produced during the incubation.

Each condition was tested in triplicates: 8 - 10 islets from each condition were handpicked into each of three $1.5 \mathrm{~mL}$ Eppendorf tubes and covered with $500 \mu \mathrm{L}$ of either $3.3 \mathrm{mM}$ or $16.7 \mathrm{mM} \mathrm{KRB}$ (as outlined in Figure 2.9). All tubes were then incubated for $1 \mathrm{~h}$ in a shaking incubator $\left(100 \mathrm{rpm}\right.$ at $\left.37^{\circ} \mathrm{C}\right)$. At the end of the incubation, all tubes were mixed and spun in a microcentrifuge (300 $\mathrm{g}$ for $30 \mathrm{~s}$ ) and all supernatant transferred into a new Eppendorf tube and stored at $-20{ }^{\circ} \mathrm{C}$ prior to insulin measurement. In the serial setup of the experiment (Figure 2.9B), the islet previously incubated at $3.3 \mathrm{mM} \mathrm{KRB}$ were now covered by $500 \mu \mathrm{L}$ of $16.7 \mathrm{mM} \mathrm{KRB}$ and incubated for another hour at $37^{\circ} \mathrm{C}$ while shaking, then mixed, centrifuged and the supernatant taken out into a new Eppendorf tube. At the end of the experiment, all islet pellets were frozen at $-80{ }^{\circ} \mathrm{C}$ for potential DNA quantification for the purposes of normalising the insulin data to the amount of cells.

Insulin concentrations from the supernatant were measured by the Core Biochemical Assay Laboratory (supported by the MRC MDU Mouse Biochemistry Laboratory [MRC MC UU 12012/5]) using an electrochemiluminescent sandwich immunoassay from MesoScale Discovery. 

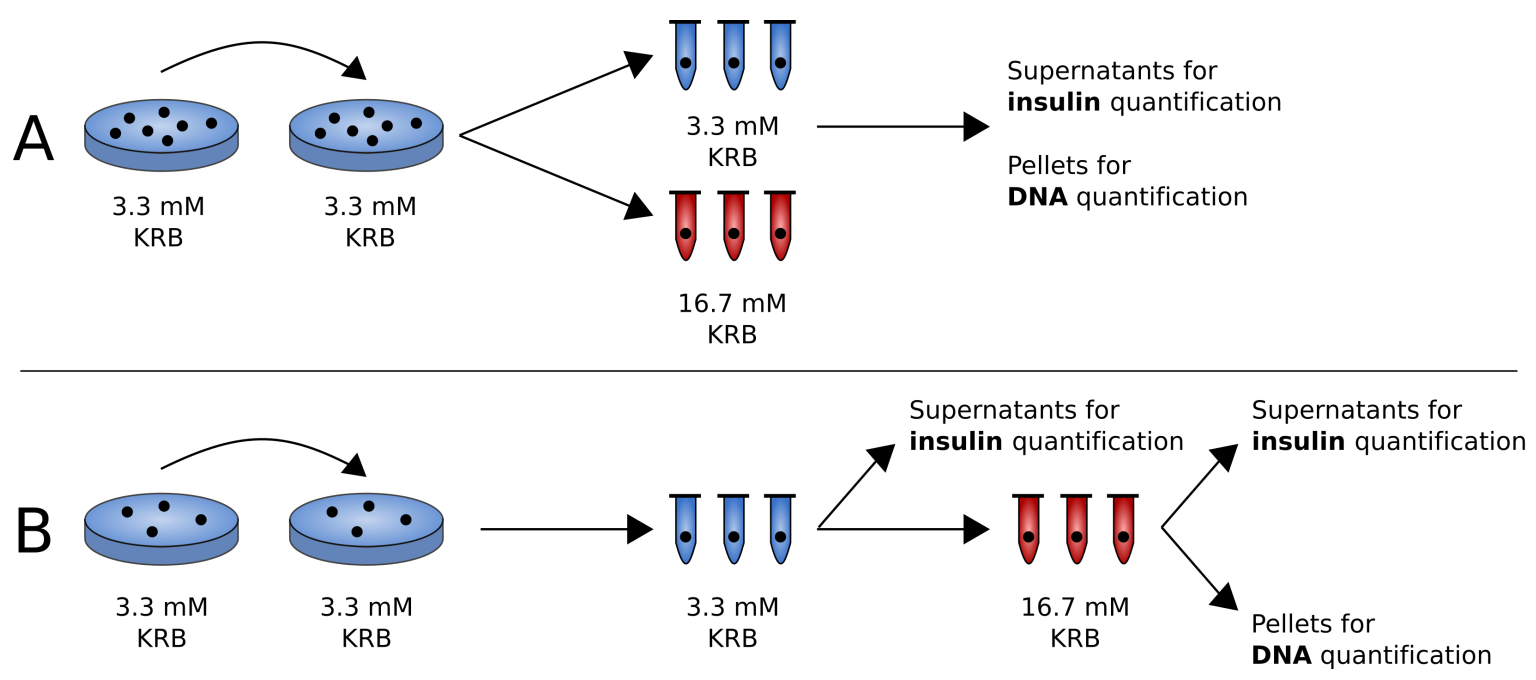

Fig. 2.9 Glucose stimulation assay protocol. Each black circle represents $\sim 10$ pancreatic islets; low glucose $(3.3 \mathrm{mM}) \mathrm{KRB}$ shown in blue, high glucose $(16.7 \mathrm{mM}) \mathrm{KRB}$ shown in red. (A) Parallel set-up of the glucose stimulation assay: starting with $\sim 70$ islets, $8-10$ islets are picked into triplicate tubes with $3.3 \mathrm{mM} \mathrm{KRB}$ and triplicate tubes with $16.7 \mathrm{mM} \mathrm{KRB}$ and incubated in parallel. (B) Serial set-up of the glucose stimulation assay: starting with $\sim 40$ islets, $8-10$ islets are first picked into triplicate tubes with $3.3 \mathrm{mM} \mathrm{KRB}$ and after retrieving supernatant post-incubation, the same islets are reused for the incubation in $16.7 \mathrm{mM}$ KRB. KRB $=$ Krebs-Ringer Bicarbonate buffer.

In Appendix D, insulin concentrations were normalised to DNA content. DNA from the pellets was extracted using the GenElute ${ }^{\mathrm{TM}}$ Mammalian Genomic DNA Miniprep Kit (Sigma-Aldrich) according to manufacturer instructions and DNA stored at $-20{ }^{\circ} \mathrm{C}$ until quantification. Quantification was done using Quant-iT ${ }^{\mathrm{TM}}$ PicoGreen $^{\mathrm{TM}}$ dsDNA Assay Kit (ThermoFisher Scientific) in 96-well flat-bottomed black polystyrene microplate (Corning) and the absorbance measured using FLUOstar Optima plate reader (BMG Labtech) using $480 \mathrm{~nm}$ excitation and emitted light detected at $520 \mathrm{nM}$ wavelength. DNA concentrations were determined based on a standard curve in each experiment. Insulin concentrations in Chapter 5 were not normalised to DNA based on optimisation in the Appendix D.

\subsubsection{Mitochondrial DNA oxidation assay}

Using quantitative Polymerase Chain Reaction (PCR) targeting mitochondrial DNA (mtDNA), the level of oxidative damage (such as strand breaks, oxidised bases or abasic sites) within mitochondria can be measured. Such damage to DNA stops polymerase from progressing along the DNA strand, thus reducing the amplification of long target ( $\sim 10 \mathrm{kbp})$ measured by the quantitative PCR. To control for the mtDNA copy number, a second short target ( $\geq 200 \mathrm{bp}$ ) is amplified in a parallel reaction and used for normalising the data. 
DNA was isolated from fresh or thawed pancreatic islets using GenElute ${ }^{\mathrm{TM}}$ Mammalian Genomic DNA Miniprep Kit (Sigma-Aldrich) according to manufacturer instructions and DNA stored at $-20{ }^{\circ} \mathrm{C}$ until the assay. Before the assay, the samples were thawed on ice and DNA concentration measured using NanoDrop ${ }^{\mathrm{TM}} 8000$ Spectrophotometer (ThermoFisher Scientific). Samples were then diluted to $3 \mathrm{ng} / \mathrm{mL}$ for the assay.

Table 2.8 Components of the PCR mixture

\begin{tabular}{llll}
\hline Component & Stock & Final concentration & Supplier \\
\hline Buffer & $10 \times$ & $1 \times$ & TaKaRa \\
BSA & $10 \mathrm{mg} / \mathrm{mL}$ & $100 \mathrm{ng} / \mathrm{mL}$ & New England Biolabs \\
dNTPs & $10 \mathrm{mM}$ & $200 \mu \mathrm{M}$ & TaKaRa \\
Forward primer & $10 \mu \mathrm{M}$ & $200 \mathrm{pM}$ & Sigma \\
Reverse primer & $10 \mu \mathrm{M}$ & $200 \mathrm{pM}$ & Sigma \\
Mg(OAc) $)_{2}$ & $25 \mathrm{mM}$ & $1 \mathrm{mM}$ & TaKaRa \\
LA Taq polymerase & $25 \mathrm{U}$ & $1 \mathrm{U}$ & TaKaRa \\
\hline
\end{tabular}

Notes: Primer sequences for mouse: Forward (5'-GCC AGC CTG ACC CAT AGC CAT AAT-3'), Reverse for short target (5'- GCC GGC TGC GTA TTC TAC GTT A -3') and Reverse for long target (5'-GAG AGA TTT TAT GGG TGT AAT GCG G-3').

For PCR, a mastermix of all components was prepared as per Table 2.8, with the exception of the polymerase which was added separately. Reaction was performed in PCR tubes and each reaction consisted of $5 \mu \mathrm{L}(15 \mathrm{ng})$ of DNA template from samples, $35 \mu \mathrm{L}$ of PCR mastermix and $1 \mathrm{U}$ of TaKaRa LA Taq polymerase. Reactions were performed in duplicates for each sample and one of the control samples was also amplified at $50 \%$ concentration to check whether the final concentration of amplified DNA lies in a linear range and nontemplate control was included. Reactions were then performed using programmes outlined in Table 2.9. Number of cycles for short and long target were optimised for our tissue type.

PCR products were quantified using Quant-iT ${ }^{\mathrm{TM}}$ PicoGreen ${ }^{\mathrm{TM}}$ dsDNA Assay Kit (ThermoFisher Scientific) according to manufacturer's instructions. Size of the PCR product was also checked by running a $0.8 \%$ agarose gel. For the analysis, all samples were corrected to the non-template control and normalised to amplification of the short target.

\subsubsection{Cyclic AMP release assay}

Fresh or thawed pancreatic islets (150-200 islets per condition) were dissociated into single cells using 5 min incubation with $0.05 \%$ trypsin $+0.53 \mathrm{mM}$ EDTA (Sigma-Aldrich) and mechanical disruption with $\mathrm{p} 1000$ pipette. Dissociation was stopped by addition of complete medium containing FCS and cells were counted using hemocytometer. 
Table 2.9 PCR programmes for long and short targets

\begin{tabular}{|c|c|c|c|}
\hline Cycles & Denaturation & Annealing & Extension \\
\hline \multicolumn{4}{|c|}{ Short target } \\
\hline $\begin{array}{l}1-21 \\
22\end{array}$ & $\begin{array}{l}94^{\circ} \mathrm{C} \text { for } 30 \mathrm{~s} \\
94^{\circ} \mathrm{C} \text { for } 10 \mathrm{~min}\end{array}$ & $64^{\circ} \mathrm{C}$ for $45 \mathrm{~s}$ & $72^{\circ} \mathrm{C}$ for $45 \mathrm{~s}$ \\
\hline \multicolumn{4}{|c|}{ Long target } \\
\hline $\begin{array}{l}1 \\
2-24 \\
25\end{array}$ & $\begin{array}{l}94^{\circ} \mathrm{C} \text { for } 1 \mathrm{~min} \\
94^{\circ} \mathrm{C} \text { for } 15 \mathrm{~s} \\
94^{\circ} \mathrm{C} \text { for } 10 \mathrm{~min}\end{array}$ & \multicolumn{2}{|c|}{$64{ }^{\circ} \mathrm{C}$ for $12 \mathrm{~min}$} \\
\hline
\end{tabular}

Measurements of cAMP were performed with the help of Dr. Kerry Barkan at the Department of Pharmacology, University of Cambridge, using LANCE ${ }^{\circledR}$ cAMP Detection Kit (PerkinElmer) in accordance with the manufacturer's instructions. The principle of the assay is explained in Figure 2.10. Duplicate or triplicate wells were prepared for each condition and each point on the standard curve. Correct amounts of single cell suspensions (4000 cells per well) were resuspended in the appropriate volume of antibody mix (Alexa Fluor ${ }^{\circledR}$ 647(AF647) anti-cAMP antibody in stimulation buffer (HBSS, 5 mM HEPES, 0.5 mM IBMX, $0.1 \%$ BSA, pH 7.4) and seeded in a white 384-well Optiplates (PerkinElmer). Forskolin, GLP1(7-26)amide, cAMP standard or DMSO control were diluted in stimulation buffer to the appropriate concentration.

For samples, $5 \mu \mathrm{L}$ per well of cell suspensions with antibody were added to the wells, together with $5 \mu \mathrm{L}$ of tested compound (DMSO for blank, $10^{-4} \mathrm{M}$ forskolin, $10^{-5}$ or $10^{-6} \mathrm{M}$ GLP1(7-36)amide) and incubated for $30 \mathrm{~min}$ at room temperature. $10 \mu \mathrm{L}$ of detection solution containing europium-labelled streptavidin and biotin-cAMP in detection buffer was then added in each well and samples were further incubated for $60 \mathrm{~min}$ at room temperature. After 60 min, measurement was taken using a Mithras LB 940 microplate reader (Berthold Technology); excitation $340 \mathrm{~nm}$ and emission $665 \mathrm{~nm}$.

\subsection{Imaging}

\subsubsection{Confocal microscopy}

Imaging of whole pancreatic islets or islet sections stained with combinations of fluorescent dyes or loaded with fluorescent compounds was performed using the Leica Sp5 (Leica Microsystems) inverted confocal microscope (NIHR Cambridge BRC Cell Phenotyping Hub). 

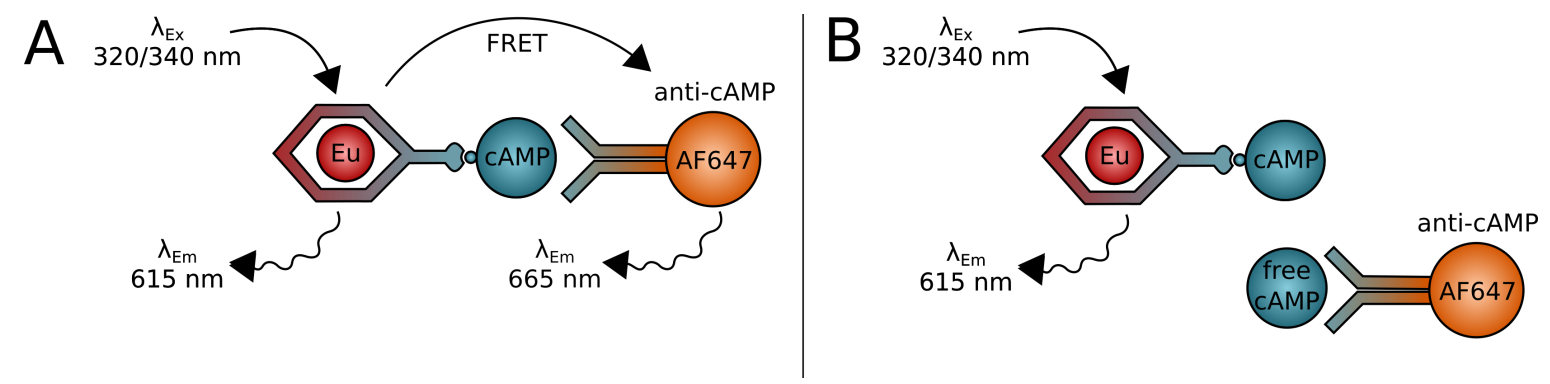

Fig. 2.10 Cyclic AMP assay principle. (A) In the absence of free cAMP, europium-labelled streptavidin bound to biotin-cAMP tracer binds to the cAMP-specific antibody, conjugated to AF647. Europium compound acts as an energy donor and upon excitation, transfers the excitation energy through FRET onto the AF647 fluorophore, which then emits fluorescence at wavelength of $665 \mathrm{~nm}$. (B) In the presence of free cAMP produced by cells, the europium-labelled streptavidin bound to biotin-cAMP is competing with the free cAMP for binding to the AF647-conjugated anti-cAMP antibody. This prevents the FRET and results in decrease of fluorescence emission at $665 \mathrm{~nm}$ depending on the concentration of free cAMP. AF647 = Alexa Fluor ${ }^{\circledR} 647$ fluorescent conjugate; $\mathrm{cAMP}=$ cyclic $\mathrm{AMP} ; \mathrm{Eu}=$ europium; FRET $=$ fluorescence resonance energy transfer; $\lambda_{\mathrm{Ex}}=$ excitation wavelength; $\lambda_{\mathrm{Em}}=$ emission wavelength.

Detection band widths were set for each fluorochrome according to its spectral properties and the effect of spectral overlap was minimised by choice of compatible fluorochrome combinations, avoiding simultaneous detection of several fluorochromes and decreasing the detection band widths if necessary. Collection of images and some simple pre-processing steps were performed using the LAS X software (Leica Microsystems).

\subsubsection{Multi-photon microscopy}

Pancreatic islets stained with a combination of fluorescent dyes were imaged using the TriM Scope II (LaVision BioTec) upright 2-photon scanning fluorescence microscope by Kevin O'Holleran at the Cambridge Advanced Imaging Centre (University of Cambridge). Different dyes were detected by using appropriate set of wide bandpass emission filters.

\subsubsection{Light sheet fluorescence microscopy}

Imaging on a light sheet microscope was done in collaboration with Till Moreth from the Goethe University in Frankfurt. Pancreatic islets stained with combination of fluorescent dyes were embedded in $1 \%$ low melting agarose and drawn into a glass capillary. Once the agarose solidified, the capillary was inserted into the holder of the microscope and imaged using the Zeiss Lightsheet Z.1 (Zeiss). The excitation/emission wavelengths were 405/415 
for Hoechst 33342, 488/498 for FDA and 561/571 for PI. Images were processed by Till Moreth.

\subsubsection{Raman spectroscopy}

For a calibration curve, solutions of increasing trehalose concentrations were prepared from D-(+)-trehalose dihydrate (Sigma Life Sciences) in distilled water by serial dilutions.

Human osteosarcoma SAOS-2 cell line was used for the proof-of-concept Raman measurements of intracellular treahalose concentration. The cells were cultured in culture-treated T25 and T75 flasks (ThermoFisher) in complete medium consisting of DMEM + $10 \%$ FCS $+1 \%$ Penicillin/Streptomycin at $37{ }^{\circ} \mathrm{C}, 5 \% \mathrm{CO}_{2}$ humidified atmosphere. Confluence of cells was checked regularly and medium was exchanged every $2-3$ days. When confluence reached about $80 \%$, cells were passaged (every $3-4$ days). This was done by washing cells in preheated PBS twice and incubation with $0.05 \%$ trypsin-EDTA (Sigma Aldrich) for 5 min at $37^{\circ} \mathrm{C}$. Once the cells had detached, the trypsin activity was stopped by adding an equal volume of complete culture medium. Cells were split in 1:4 ratio and brought into fresh complete culture medium.

SAOS-2 cells were used for the Raman spectroscopy experiment in an early passage. At a confluence stage, the cells were seeded into a 6-well culture-coated plate (ThermoFisher) with Quartz substrate glass slide on the bottom for the cells to grow on. Cells were cultured until $70 \%$ confluence was reached on the Quartz slides. The culture medium was then exchanged for the tested solution ( $200 \mathrm{mM}$ trehalose, $200 \mathrm{mM}$ trehalose $+100 \mu \mathrm{g} / \mathrm{mL} \mathrm{PP}-50$, control culture medium) and incubated for $6 \mathrm{~h}$ at $37^{\circ} \mathrm{C}$. At the end of this incubation, the cells were washed twice with PBS to remove any extracellular trehalose and fixed using $4 \%$ paraformaldehyde solution for $15 \mathrm{~min}$. After the fixation, cells were covered with PBS and kept at $4{ }^{\circ} \mathrm{C}$ until analysis.

Ramans spectra were measured using Acton SpectraPro SP 2300 spectroscope (Princeton Instruments), connected to WiTec alpha300 M+ Raman confocal microscope (WiTec) using the $782.92 \mathrm{~nm}$ excitation laser. Averaged Raman spectra across 10 cells were recorded for each condition (each consisting of 30 individual Raman spectra). The optimal Raman band to identify trehalose is at $844 \mathrm{~cm}^{-1}$ and all subsequent analysis was performed using this band. Analysis was performed using Origin Software (OriginLab). All Raman measurements and data analysis was performed by Dr. Jakub Surmacki at the Cavendish Laboratory, University of Cambridge. 


\subsection{Statistical analysis}

Statistical analysis was performed using the GraphPad Prism software (version 8.0.1). Power analysis was not performed for in vitro experiments since it was expected that each optimisation step would provide only an incremental increase in the sample viability and therefore the recommended sample size for such small effect size would be very high and impractical (regarding the limited availability of human tissue and mouse pancreatic islets). The aim was to use sample size of 3-5 for these optimisation experiments and then perform a full power calculation for the final comparison of the standard and fully optimised protocol. For in vivo experiments, pilot experiments were performed which ascertained likely effect size and variance, upon which power calculations were performed to confirm group sizes.

For in vitro datasets with a sample size smaller than 5, samples of the same type from multiple experiments (for example all splenocyte DMSO samples with the incubation time of $0 \mathrm{~h}$ and cell density of $10^{7}$ cells $/ \mathrm{mL}$ ) were pooled together for test of consistency with Gaussian distribution. By such method, datasets with sample size between 7 and 10 were created, which were suitable for performing a normality test. Nevertheless, I am aware that the with such small sample size there is a risk that some deviations from normality will not be detected. Normality of the distribution was assessed by Shapiro-Wilk test on a three dataset of the flow cytometry of splenocytes which have all passed the normality test for the cryoprotectant DMSO, trehalose alone and trehalose with PP-50. Based on this result, Gaussian distribution was assumed for all the smaller datasets which could have not been tested. For large sample sizes the normality tests were performed using Shapiro-Wilk test.

For normally distributed data, two-tailed paired t-test was used for comparison of two groups. To compare three or more groups, one-way or two-way ANOVA (analysis of variance), followed by Tukey's honestly significant differences (HSD) test (controlling for multiple comparisons) was used if three or more groups were compared to each other, Sidak's correction if two groups in two-way ANOVA were compared to each other and Dunnett's test if groups were compared to a single control group. Results were considered statistically significant if $\mathrm{p}$-value $\leq 0.05$.

Results obtained from viability staining of pancreatic islets at $24 \mathrm{~h}$ after thawing followed bimodal distribution. Although more exact model could be developed, for the purposes of this thesis, calculation of means using standard methods was used as an approximation to determine average viability of each sample. Once multiple experimental repeats were pooled together, these means then followed Gaussian distribution and were analysed using standard methods described above. 


\section{Chapter 3}

\section{Viability assessment of mononuclear cells and pancreatic islets}

\subsection{Introduction and aims}

Reporting the viability of cell samples is crucial in good research practice. Standard viability assessment methods for both single cells and multicellular aggregates can be accurate but also present a risk of over- or underestimation of viability if important details are neglected. Particularly in cryobiology where the comparison of post-thaw survival of multiple cryopreservation protocols is performed, it is critical for the viability assessment to be accurate, unbiased and reproducible.

Section 3.2 focuses on identification of the requirements for a reliable cryosurvival assessment method with regards to the field-specific needs of cryobiology. These parameters are then taken into consideration to modify viability assessment method for mononuclear cells in Section 3.3. Special attention is paid to flow cytometry and its adaptations to allow for unbiased enumeration of cells and cell subsets in cryobiological samples.

Viability assessment of multicellular aggregates such as pancreatic islets is more complex, given their larger size, complicated architecture and unknown parameters of the staining process. Clinical assessment of pancreatic islet viability is done by rapid two-colour fluorescent staining and imaging on a wide-field microscope. It has been long known that islet viability measured using this method does not correlate with their in vitro and in vivo function [92] and in Section 3.4, some of the possible reasons for this were identified by studying diffusion properties of islets and investigating different imaging methods. 


\subsection{Suitable viability assessment method for cryobiology}

For single cells, the choice of viability assessment method is often trypan blue staining or flow cytometry. Majority of research fields focus on identification and characterisation of cells which are alive and excluding dead cells from the flow cytometric analysis is entirely acceptable as long as the cell death rate is low. In cryobiology, however, ignoring dead cells and debris is not appropriate. Different cryopreservation protocols produce different ratios of alive cells, dead cells and debris and these proportions should be quantified in studies pursuing freezing methods comparison.

Quantification of dead cells and debris, however, is problematic. As cells die, they can disintegrate into many fragments of debris. One potential solution is to report absolute numbers of live cells in comparison with other samples within the same experiment, or better, with the absolute number of cells in the sample before freezing (Figure 3.1). Reporting absolute numbers/aggregate sizes and comparison with pre-freeze sample provides essential information about the potential clinical application of the cryopreservation method, because specific target number of (viable) cells are needed to achieve a therapeutic effect in a patient.

In some cases, mixed populations of cell types are cryopreserved (B cells, T cells, haematopoietic progenitors in BM mononuclear cells; $\alpha-, \beta-, \delta$-cells in pancreatic islets etc.). Cryosurvival of certain cell type may be of particular interest (e.g. CD34 ${ }^{+}$for bone marrow transplantation) but not all freezing protocols will be equally efficient for all cell types. A cryopreservation method most suitable for progenitors may not, for example, be the right choice if we are looking to preserve T cells for CAR-T cell therapies. An ideal viability assessment method can detect the differences in survival of cell subsets and hence inform optimisation of cell subset-specific freezing protocols.

Finally, the cryobiology field would benefit from standardisation of cryosurvival reporting method. Currently, comparison of publications from different groups are often hindered by the fact that some groups report viability as percentage of all cells, some percentages of certain cell subsets, some as absolute numbers.

Taking all these considerations into account, key cryobiology-specific needs for both single cell and multicellular aggregates viability assessment method therefore include:

1. Accurate identification of not only live cells, but also dead cells and debris

2. Unbiased method of cell/cell cluster enumeration

3. Accurate comparison of the cell samples before freezing and after thawing

4. Information about survival of cell subsets within mixed cell populations 


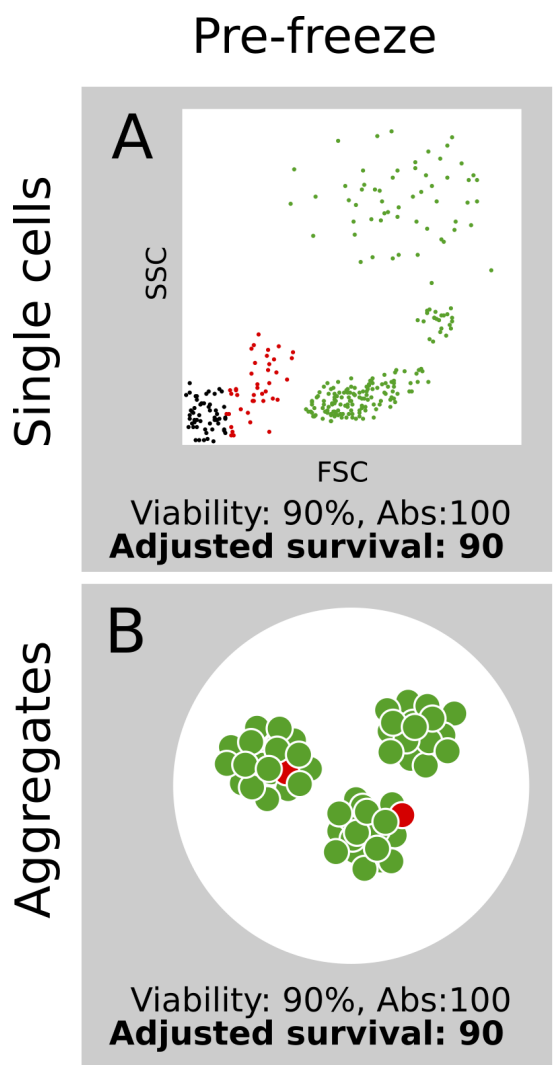

Post-thaw C1
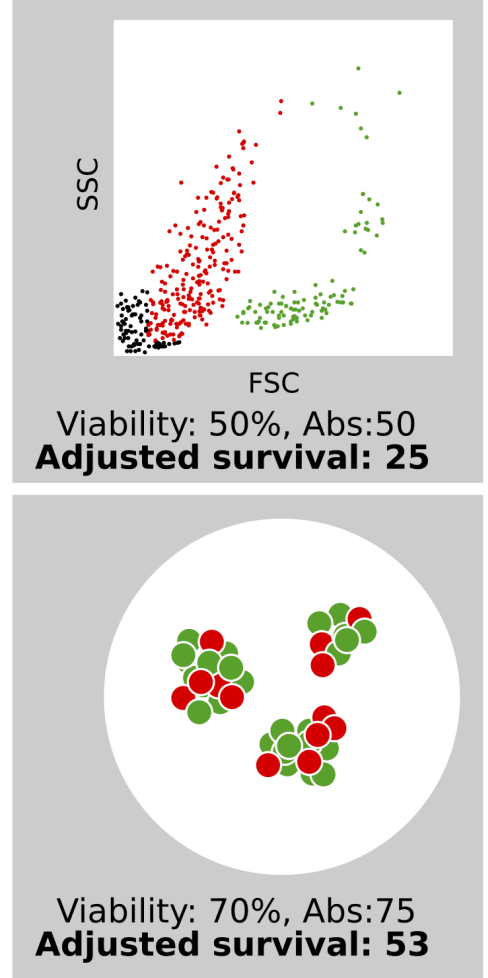

Post-thaw C2
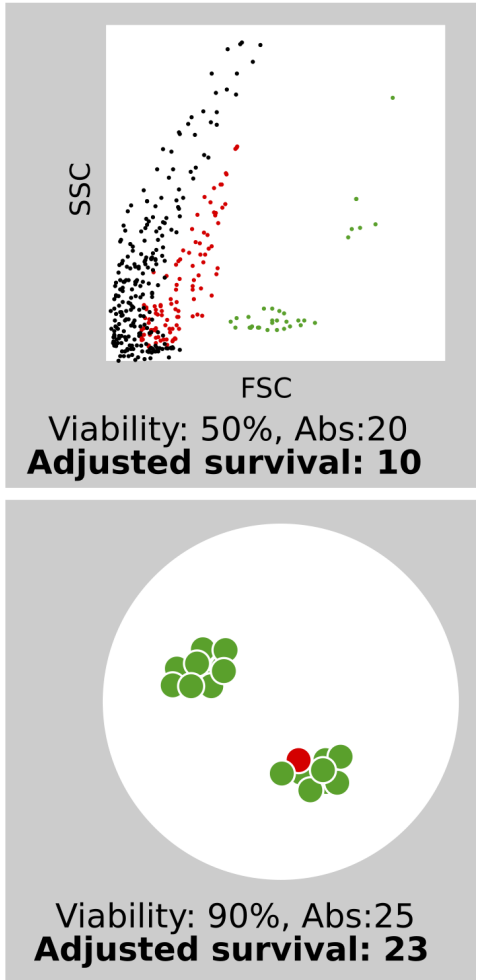

Fig. 3.1 Viability assessment considerations for single cells and multicellular aggregates. Live cells are shown in green, dead cells in red, debris in black. (A) A schematic of flow cytometric analysis of mononuclear cell viability in fresh sample and two cryopreserved samples $(\mathrm{C} 1, \mathrm{C} 2)$. Reporting percentage of viability as the only outcome can be misleading as the amount of debris is often ignored. In this example, the $\mathrm{C} 1$ and $\mathrm{C} 2$ protocols produce the same ratio of live and dead cells but in $\mathrm{C} 2$, many cells have been disintegrated into debris which was not accounted for in the viability measurement. Appropriate way would be to report the absolute cell counts in each sample and to compare the outcome to the same sample before freezing. (B) A schematic of microscopic analysis of pancreatic islets stained with dual viability stain. In this case, the Abs parameter must take into account both the number of cell clusters and their size. In some cryopreservation methods, the size of islets would be largely unchanged but viability is affected $(\mathrm{C} 1)$ while other protocols will be be more prone to islet disintegration but will preserve viability well $(\mathrm{C} 2)$. Abs = Absolute number of cells in the sample; $\mathrm{FSC}=$ forward scatter; SSC $=$ side scatter; Viability $=\%$ viable cells of all cells; Adjusted survival $=$ Viability $\times$ Abs. 
5. Replicable protocols enabling comparison of cryopreservation protocols between labs

\subsection{Cryosurvival assessment for mononuclear cells}

In this project, flow cytometry was chosen over trypan blue staining as a viability assessment method for its adaptability, accuracy and possibility to identify subsets in mixed cell populations. Classical flow cytometry protocols can be easily adapted to provide not only percentages of dead and surviving cells but also their absolute numbers, simplifying the comparison of multiple groups.

\subsubsection{Gating using light-scattering properties versus fluorescence}

In flow cytometry, population of interest often has characteristic shape and position when plotted in forward scatter (FSC) against side scatter (SSC) view. Some studies consider gating based only on light scatter characteristics sufficient for selection of viable cells, an approach which has been shown inadequate or even impossible in many cases [170]. Common recommendation in flow cytometry is to also apply a forward scatter threshold to eliminate debris from the analysis.

There are several issues with assessing cryopreserved cells based on light scatter properties. During freezing, cells undergo substantial osmotic and thermal stress which can change their size [88], intrinsic morphology and cell membrane characteristics. All these parameters have influence on light scattering parameters of the cells, and gating based solely on FSC and SSC characteristics, such as in the case of 'classical' gating strategy (Figure 3.2A, B and $\mathrm{C}$ ), could therefore result in unwanted exclusion of viable cells from the analysis and loss of information about dead cells and debris. Partial overlap of live cells, dead cells and debris in their light scatter properties can also make their clear discrimination impossible [170].

To avoid these issues, fluorescence can be used to accurately gate on the cell population of interest. Membrane integrity stains (such as 7-AAD) are traditionally used for determination of cell viability. Syto ${ }^{\circledR} 13$ dye which binds to nucleic acids, can be used to stain all nucleated cells (both alive and dead). Combination of Syto13 and membrane integrity dye, can therefore be used to effectively exclude debris and erythrocytes from the analysis, as suggested by Reardon et al. [88].

In this project, this strategy of combining a viability dye and a nuclear dye was adopted. Minimal FSC threshold was set on the flow cytometer to prevent loss of information about any events, including debris. The suggested gating strategy is shown on the example of mononuclear cells in Figure 3.2D, E and F. The identified population of alive nucleated (AN) 

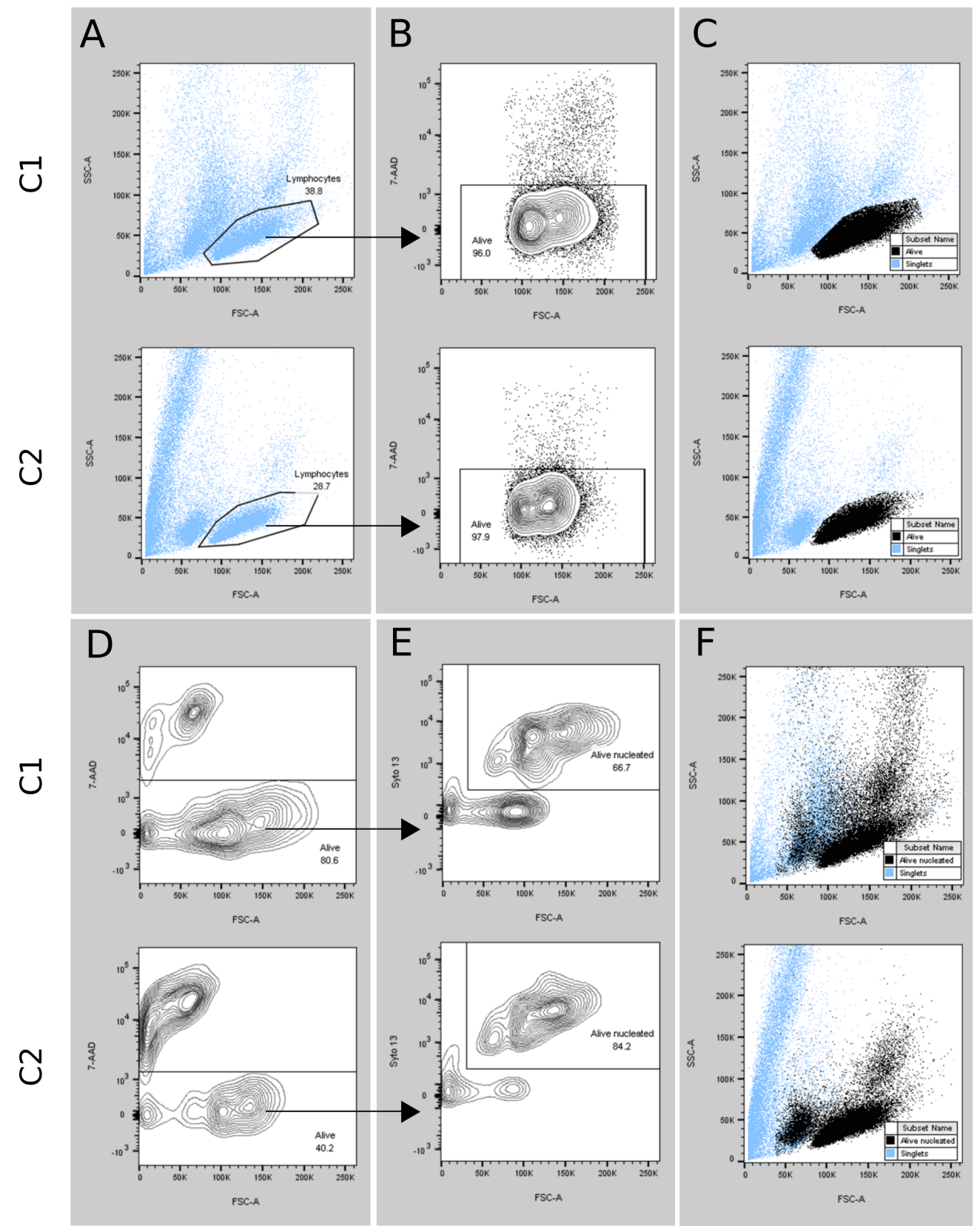

Fig. 3.2 Comparison of gating strategies for viability assessment of different cryopreservation methods (C1 and C2). (A) First step of 'classical' gating strategy - gating on the cell population using FCS vs. SSC plot. (B) Second step of 'classical' gating strategy - gating on viable cells using a plot with viability marker (in this case 7-AAD). (C) Position of the viable cells on the FSC vs. SSC plot after the 'classical' gating. (D) First step of optimised gating strategy - gating on negative population using a plot with viability marker (in this case 7-AAD). (E) Second step of optimised gating strategy - gating on positive population using a plot with nucleated cell marker Syto13. (F) Position of alive nucleated cells (black) on the FSC vs. SSC plot after the optimised gating. Blue events represent debris, erythrocytes and dead cells. FSC = forward scatter; SSC = side scatter; 7 - $\mathrm{AAD}=7$-aminoactinomycin $\mathrm{D}$. 
cells includes all cells (lymphocytes, granulocytes and other nucleated cells) regardless of their light scatter properties which is in stark contrast to the rather artificial border created by the 'classical' technique (Figure 3.2A, B, and C).

\subsubsection{Quantification of viability and cryopreservation efficacy}

Accurate detection of viable cells in the sample does not necessarily lead to correct reporting of the outcome of cryopreservation. In cryobiology publications, it is often not specified which denominator was used to report the \% viability/survival, although there are multiple possibilities:

1. \% of total number of recorded events (often with unspecified FSC threshold)

2. \% of all cells with position typical for the particular cell type on FSC/SSC plot

3. $\%$ of all cells positive for a marker characteristically expressed by all the analysed cells (e.g. $\mathrm{CD} 45^{+}$for mononuclear cells)

As explained in Section 3.2, is it advantageous to report absolute numbers of cells which in fact eliminates the need for choosing the 'correct' denominator. However, where the absolute count was not available in this project, following approximation relating to the number of all singlet events was used to calculate viability as \% of alive nucleated (AN) cells (Syto13 ${ }^{+}, 7-\mathrm{AAD}^{-}$with the assumption that the amount of debris in different samples negatively correlates with the success of cryopreservation):

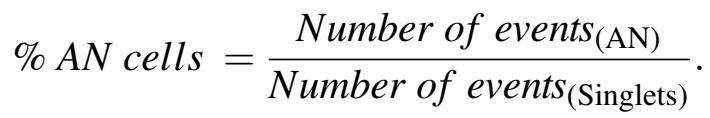

To obtain absolute cell counts in flow cytometry, counting beads can be added to the stained cell sample and calculations performed as described in the Methods section 2.5.1. The cell count should ideally be performed just before cryopreservation (by removing a small volume from the cryovial before freezing) and at the end of the thawing procedure (Figure 3.3A). If the counted cells are stained with the above mentioned viability and nuclear dyes, the efficacy of the cryopreservation $\left(\mathrm{CE}_{\text {total }}\right)$ can then be calculated using the absolute numbers of $\mathrm{AN}$ cells (further referred to as $\mathrm{Abs}_{\mathrm{AN}}$ ) by following formula:

$$
C E_{(\text {total })}=\frac{A b s_{(\mathrm{AN})} \text { after thawing }}{A b s_{(\mathrm{AN})} \text { before freezing }}
$$




\section{Survival of all cells}

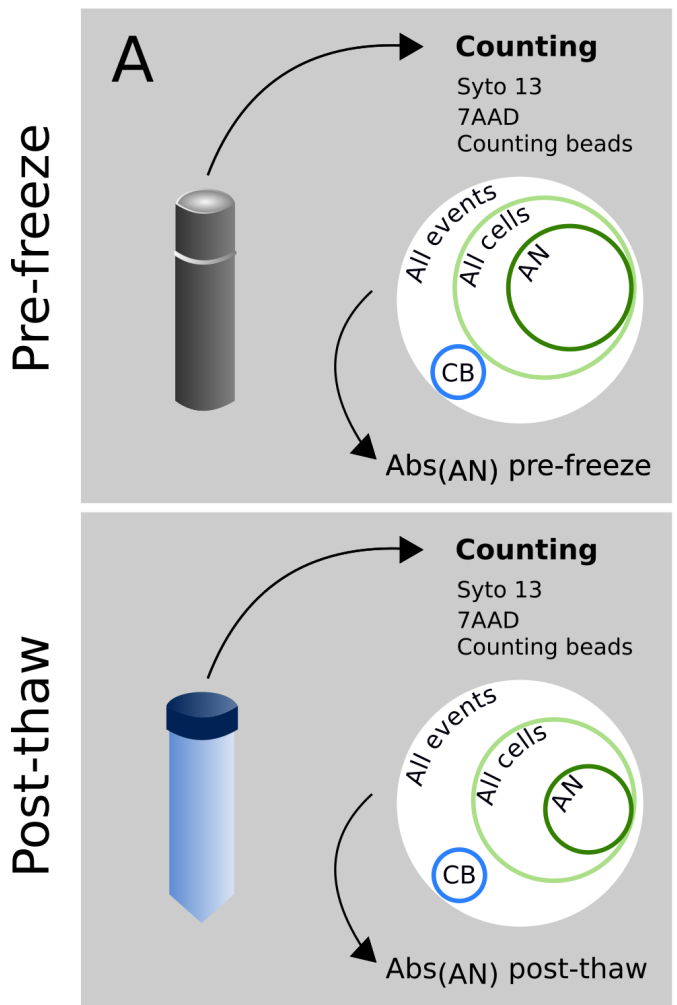

\section{Survival of cell subsets}

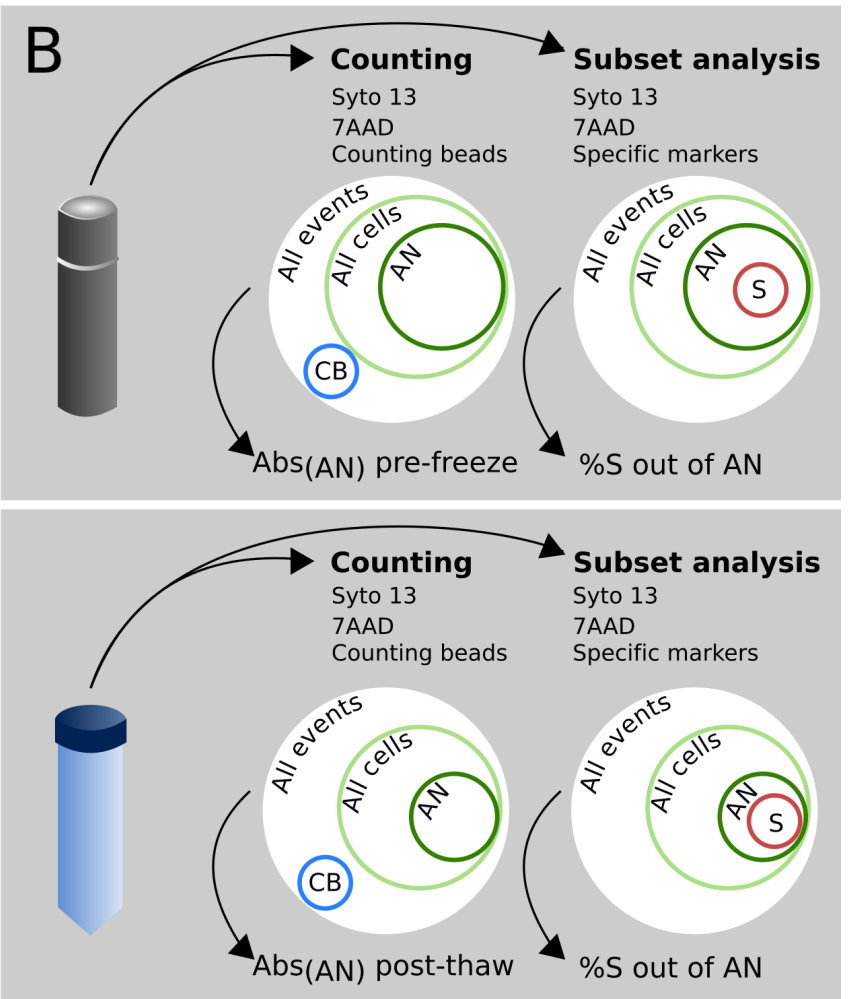

Fig. 3.3 Method of quantification of absolute number of cells and cell subsets surviving cryopreservation. In a form of Venn diagram, the strategy is visually demonstrated. (A) Small known volume of cell suspension is transferred from the cryovial before freezing into the BD Trucount ${ }^{\mathrm{TM}}$ tube and using no-wash protocol to avoid cell losses, stained with Syto13 and 7-AAD. Same procedure is then repeated post-thaw and absolute number of alive nucleated cells is compared with the pre-freeze count. (B) Prior to freezing, small known volume of cell suspension is transferred into a counting tube (same as (A)) and into an additional staining tube where the subset analysis is done using cell subset specific markers along with Syto13 and 7-AAD. Using the absolute number of alive nucleated cells and $\%$ of the cell subset as a proportion of alive nucleated cells, absolute number of the cell subset can be calculated. When identical analysis is performed post-thaw, cryopreservation efficacy for the specific cell subset can be calculated. In the outlined example, majority of the cells in the subset $\mathrm{S}$ survived the cryopreservation, while the number of the remaining alive nucleated cells was decreased. $\mathrm{Abs}_{\mathrm{AN}}=$ absolute number of alive nucleated cells; $\mathrm{AN}=$ alive nucleated cells; $\mathrm{CB}=$ counting beads; $\mathrm{S}=$ cell subset. 


\subsubsection{Survival of cell subsets}

For an accurate quantification of cells using counting beads, washing steps must be eliminated from the staining protocol to prevent the unavoidable cell loss during centrifugation and pipetting. However, identification of cell subsets, especially of rare populations, require carefully optimised staining protocols, which almost invariably include washing steps. Their purpose is to remove any background signal from the unbound antibodies or stains in order to decrease the chance of detecting false positive events.

While washing DNA-binding dyes (such as viability dyes or Syto13) is not necessary, many conjugated antibodies or dyes cause a significant noise signal. In these cases, one strategy is to split the original sample into two (or more) tubes. First tube would contain the counting beads and no-wash staining with Syto13 and 7-ADD (or similar) would be performed. In the other tube(s), staining with other antibodies/dyes is performed along with the Syto13 and 7-AAD. Since the alive nucleated (AN, Syto13 ${ }^{+}$7-AAD ${ }^{-}$) cells can be gated in all the fluorescent panels and their absolute number is known from the counting tube, the absolute numbers of cell subsets from the other tubes $\left(\mathrm{Abs}_{\text {(subset) }}\right)$ can be related to this absolute number $\left(\operatorname{Abs}_{(\mathrm{AN})}\right)$ in the following way:

$$
A b s_{(\text {subset })}=A b s_{(\mathrm{AN})} \text { in counting beads tube } \times \frac{\text { Number of events }}{\text { (subset) }}
$$

Analogically to the equation 3.2, to calculate the efficacy of cryopreservation for a particular subset $\left(\mathrm{CE}_{\text {subset }}\right)$, the absolute numbers post-thaw must be related to absolute numbers pre-freeze:

$$
C E_{(\text {subset) }}=\frac{A b s_{\text {(subset) }} \text { after thawing }}{A b s_{\text {(subset) }} \text { before freezing }}
$$

The suggested protocol is depicted in Figure 3.3B for further clarification.

\subsection{Cryosurvival assessment for pancreatic islets}

\subsubsection{Current viability assessment method evaluation}

\section{Imaging viability across the pancreatic islet}

Because islet quality evaluation method must be reasonably quick and accessible to the clinical islet isolation laboratories, they mostly rely on simple dual fluorescent viability 

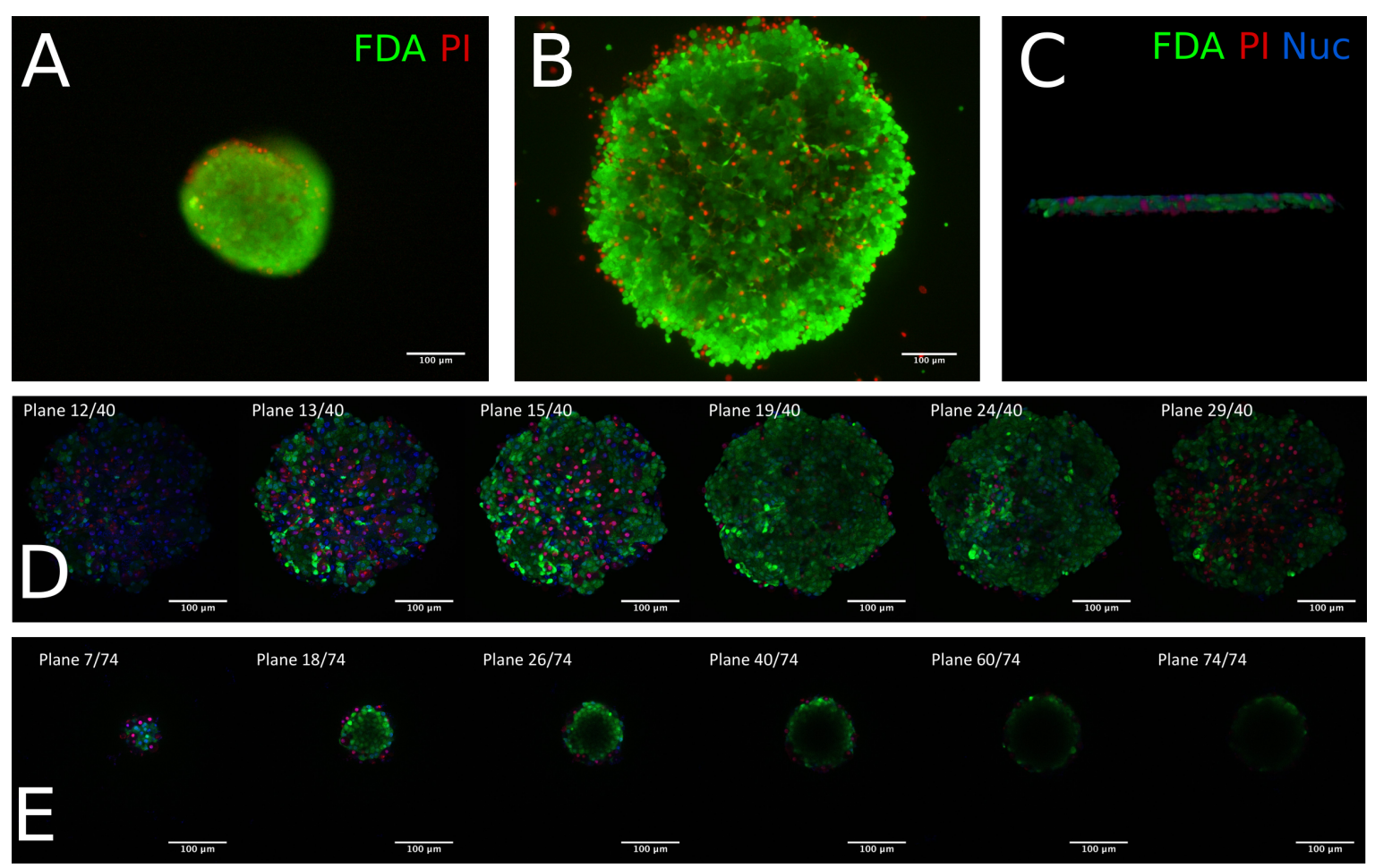

Fig. 3.4 Current imaging method for pancreatic islet viability assessment. FDA shown in green, PI in red and Hoechst 33342 in blue.(A) Pancreatic islet stained with FDA and PI imaged by a wide-field fluorescent microscope on top of a coverslip. (B) Pancreatic islet stained with FDA and PI imaged by a wide-field fluorescent microscope flattened in between two coverslips. (C) 3Dreconstruction of the flattened pancreatic islet in between two coverslips imaged on a confocal microscope (FDA/PI/H33342 staining). (D) Z-stack of the flattened pancreatic islet in between two coverslips imaged on a confocal microscope (FDA/PI/H33342 staining). (E) Z-stack of a pancreatic islet on top of a coverslip (unflattened) imaged on a confocal microscope (FDA/PI/H33342 staining). $\mathrm{FDA}=$ fluorescein diacetate; $\mathrm{PI}=$ propidium iodide; $\mathrm{H} 33342=$ Hoechst 33342.

staining and imaging on wide-field microscope (see Methods 2.6.2). Because wide-field microscopy uses non-directed light, the visible cells are located mostly at the outer layer of the stained pancreatic islets (Figure 3.4A).

This original method was initially adapted by gently placing a coverslip on top of the glass slide which led to flattening of the 3D islet into a thinner disc. This theoretically allows the majority of the cells to be detected in the plane of focus of the wide-field microscope, although the information about the original localisation of each cell within islet is lost (Figure 3.4B). Confocal microscopy demonstrated that using this method, islets are flattened to a thickness of approximately $20 \mu \mathrm{m}$, equivalent to $2-3$ layers of cells (Figure 3.4C and D).

Because current viability assessment method described above completely ignores the information about the viability of the cells localised in the centre of the pancreatic islets, 

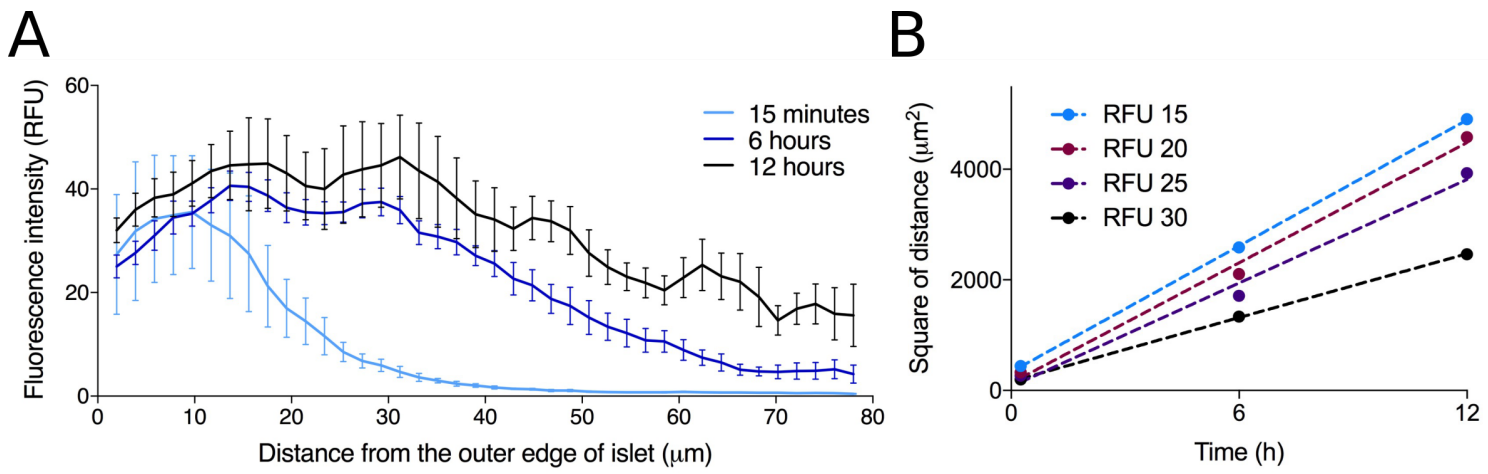

Fig. 3.5 Diffusion of nuclear dye into pancreatic islets at room temperature. (A) Staining profile of pancreatic islets incubated with Hoechst 33342 nuclear dye for 15 min, $6 \mathrm{~h}$ or $12 \mathrm{~h}(\mathrm{n}=3-5)$. (B) Linear relationship between time and square of distance shown for four different fluorescent intensities from the plot in (A). RFU = relative fluorescence intensity.

confocal microscopy was employed to image the viability of the whole islet in 3D. This would be especially crucial in order to confirm that viability of cryopreserved islets is retained in both on the periphery and in the core. Nevertheless, it was impossible to image the central cells in pancreatic islets using the confocal microscope (Figure 3.4E). Two mutually nonexclusive possible reasons were identified: insufficient staining time to allow for staining of the central cells and imaging limitations of the confocal microscopy due to characteristic properties of live islets.

\section{Diffusion of dye molecules into pancreatic islets}

Because isolated pancreatic islets are devoid of (flow in) vasculature, all molecules are transported by diffusion. The diffusion pattern of dye molecules was therefore studied to confirm whether all cells within the pancreatic islets were stained.

Depending on the nature of the molecule of interest, these either diffuse only through the extracellular spaces (non-penetrating molecules), or pass freely through cell membranes. In the case of Hoechst 33342, passage through cell membrane is possible but the diffusion by this channel will be slowed down by the 'uptake' of dye molecules by binding to the nuclear DNA. Hoechst 33342 nuclear dye was chosen over the original viability staining molecules due to the fact that it stains all cells regardless of their viability (PI would only stain small fraction of cells) and its resistance to photobleaching (FDA starts losing its intensity within seconds of excitation). The diffusion rate of Hoechst 33342 is not identical to other molecules but since all the dyes of interest are small molecules travelling in an aqueous solution, the assumption was made that the data generated is a good approximation for other dyes. 
The depth of diffusion of the dye was determined using a novel method described in Methods section 2.6.4. Initial experiments included the original staining procedure - staining at room temperature for $15 \mathrm{~min}$. Additional, longer staining times were used to characterise the diffusion rate at these conditions. As shown in Figure 3.5A, after 15 min of incubation with Hoechst 33342, only 3 - 4 outer layers of cells were stained while any cells beyond the distance of $40 \mu \mathrm{m}$ into the islet core lacked any staining. Increasing the incubation time led to higher intensity of staining in the central regions of the islets but even after $12 \mathrm{~h}$ of staining at room temperature, homogenous staining of the islets was not achieved. Because clinical and research laboratories stain pancreatic islets for very short time (typically $<15 \mathrm{~min}$ ), this finding confirms that the current clinically-used methods assess viability of the cells on the periphery of the islet, while ignoring the cells in the centre.

\subsubsection{Increasing diffusion rate of molecules into pancreatic islets}

The diffusion of molecules of one substance in another substance down its concentration gradient is described by Fick's law. If we assume a spherical pancreatic islet with a constant surface boundary and initial concentration of the surface equal 0, Fick's law can be modified (as described in detail in [171]) to get a simple relationship:

$$
t=\frac{x^{2}}{D}
$$

where $D$ is diffusivity (diffusion coefficient) of a substance specific to a mixture with another substance; $t$ is time and $x$ is distance. It follows that square distance of the penetration increases linearly with the time allowed for penetration. As shown in Figure 3.5B, the data from the diffusion experiment in Section 3.4.1 experiment follows this relationship and it can be assumed simple molecular diffusion is the main process responsible for the movement of molecules in this system.

The nature of clinical islet transplantation requires rapid assessment of pancreatic islet quality. One of the reasons why the current viability scores do not correlate with function of pancreatic islets or even clinical outcomes may be because it only takes into account fraction of the cells on the periphery of the islet. However, it appears that the diffusion of molecules at the above staining conditions is relatively slow and would require increasing the incubation time to length which would not be practical.

Diffusivity is dependent on temperature, pressure and because it is described for every pair of specific compounds, on the presence of other compounds in the system [172]. In 

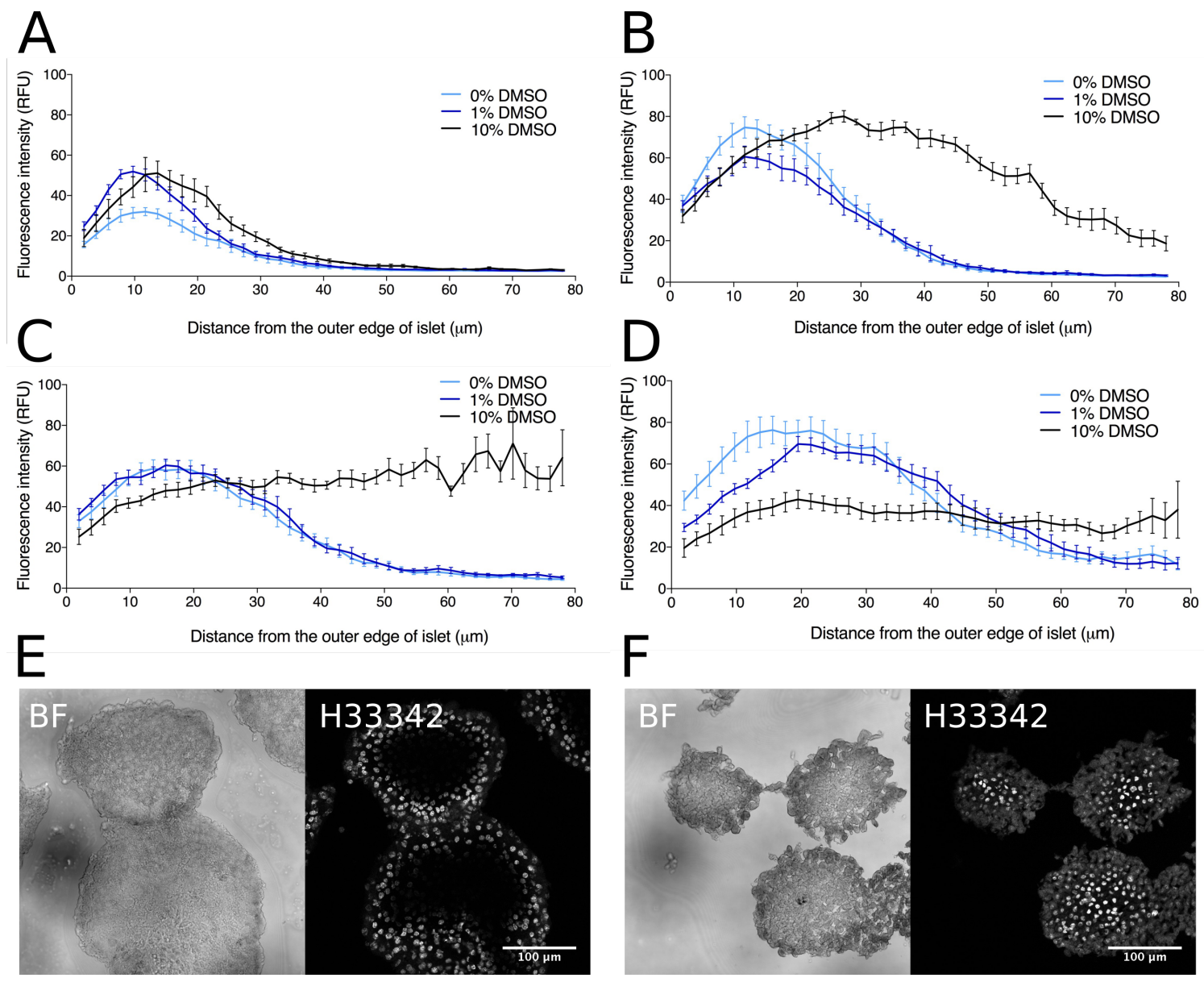

Fig. 3.6 Diffusion of nuclear dye into pancreatic islets with DMSO as a penetration enhancer. Staining profiles of pancreatic islets incubated Hoechst 33342 and $0 \%, 1 \%$ or $10 \%$ DMSO for (A) 15 min, (B) $1 \mathrm{~h}$, (C) $3 \mathrm{~h}$, (D) $6 \mathrm{~h}$ (n = 5 - 17). (E) Bright field and Hoechst 33342 fluorescence images of islets incubated with $0 \%$ DMSO for $3 \mathrm{~h}$. (F) Bright field and Hoechst 33342 fluorescence images of islets incubated with $10 \%$ DMSO for $3 \mathrm{~h}$. BF = bright field; H33342 = Hoechst 33342 .

the below sections, the influence of these parameters on increasing the rate of diffusion was studied.

\section{Effect of DMSO as a penetration enhancer}

The diffusion rate of one compound can be influenced by the concentration gradients of another compound present in the mixture. One of the compounds known for its higher diffusivity is DMSO. While current data demostrates that a dye molecule in aqueous solution can take many hours to equilibrate, Benson et al. showed that $1.5 \mathrm{~mol} / \mathrm{kg}$ DMSO can equilibrate with an islet of $150 \mu \mathrm{m}$ diameter in about $600 \mathrm{~s}$ at room temperature [72]. DMSO is also known to be a chemical penetration enhancer, meaning that it can accelerate diffusion of 
other molecules across membranes, likely due to its interactions with membrane lipids [173]. A following hypothesis was tested: addition of DMSO during the staining with Hoechst 33342 will increase the rate of diffusion of the dye.

Two concentrations of DMSO (1\% and $10 \%$ ) were tested in addition to the control. While a clear effect of $1 \%$ DMSO on the diffusion rate was not observed (Figure 3.6A, B, $\mathrm{C}$ and D), $10 \%$ DMSO enhanced the diffusion rate significantly, as shown in Figure 3.6B). Nevertheless, at $3 \mathrm{~h}$ incubation, DMSO toxicity was apparent both in the staining profiles (decrease of staining intensity close to the outer edge) and in the images (necrosis-related karyolysis at the periphery of the islets can be observed as diffused nuclear staining).

In conclusion, DMSO at $10 \%$ concentration increases the diffusion rate of the nuclear dye but leads to toxicity which makes this method unsuitable for use in viability assessment staining.

\section{Effect of hydrostatic pressure}

Diffusivities are also known to increase with pressure. Since the islets are immersed in solution during staining, the impact of increasing hydrostatic pressure on diffusion was investigated next. Hydrostatic pressure $\left(P_{h}\right)$ is a function of height of fluid column $(h)$, density of liquid $(\rho)$ and acceleration of gravity $(g)$ :

$$
P_{\mathrm{h}}=h \times \rho \times g
$$

Increasing the density of liquid would be counterproductive since the denser the liquid, the slower the diffusion (Graham's law of effusion). Changing the height of fluid column is possible but the effect will be limited by the size of the container. The most practical way to increase hydrostatic pressure in this system was therefore to increase the gravitational force $(g)$ which was achieved by centrifugation at different speeds.

Islets were incubated with the Hoechst 33342 nuclear dye for $15 \mathrm{~min}$ at room temperature with $(35 \times g$ or $105 \times g)$ or without $(0 \times g)$ centrifugation. The result suggests that there is marginal improvement in the depth of the diffusion (Figure 3.7A). The only observable effect was concentration of the dye molecules on the surface of the islet (Figure 3.7B and C).

While it is possible that an effect could be observed at longer incubation times, there is a concern that prolonged centrifugation could have an impact on the viability of the islets. Additionally, Hoechst 33342 dye (and most other dyes) may cause toxic effect to cells at high concentrations and this method appears to cause a local increase in the concentration of 

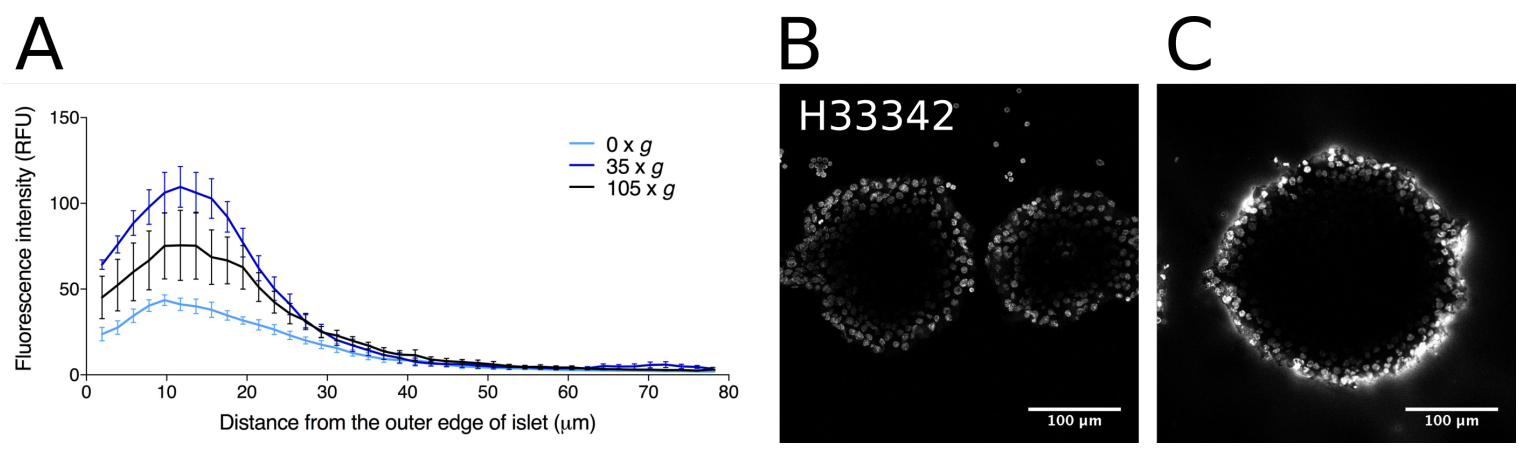

Fig. 3.7 Effect of hydrostatic pressure on diffusion of nuclear dye into pancreatic islets. (A) Staining profiles of pancreatic islets incubated Hoechst 33342 for 15 min at $0 \times g, 35 \times g$ or $105 \times g(n=6)$.

(B) Hoechst 33342 fluorescence image of islets stained with Hoechst 33342 at $0 \times g$ for 15 min.

(C) Hoechst 33342 fluorescence image of islets stained with Hoechst 33342 at $35 \times g$ for $15 \mathrm{~min}$. H33342 = Hoechst 33342 .

the dye on the surface of the islets. It was therefore concluded that this method is not suitable for accelerating diffusion in pancreatic islets.

\section{Effect of temperature}

Diffusivities of compounds are closely dependent on the temperature in the system. Higher temperatures increase the kinetic energy of molecules, making them move at higher speed. Along with incubation at room temperature, in this experiment islets were stained at $37{ }^{\circ} \mathrm{C}$.

The result from this experiment confirms the previous finding that at room temperature, even staining of pancreatic islets cannot be achieved even after $24 \mathrm{~h}$ (Figure 3.8A). At $37{ }^{\circ} \mathrm{C}$, however, pancreatic islets were homogeneously stained after only $6 \mathrm{~h}$. Staining for $24 \mathrm{~h}$ further increased the intensity of the staining throughout the islet (Figure 3.8B).

In a second experiment, shorter incubation times at $37{ }^{\circ} \mathrm{C}$ were investigated. However, after 1 or $3 \mathrm{~h}$ of staining, there was still very low staining in the central part of the islets. Six hours of incubation achieved staining in the whole volume of the islets, although the intensity was not the same across the whole section as in the previous experiment (Figure 3.8C).

To confirm that the islets stained with Hoechst 33342 for $6 \mathrm{~h}$ at $37^{\circ} \mathrm{C}$ remained viable during the assay, FDA/PI staining was performed. Although there is a small drop in viability, which can likely be attributed to the Hoechst 33342 toxicity (Figure 3.8D), it is not expected that this drop in viability would have a large effect on the outcome of the above diffusion experiments.

Since pancreatic islets can be cultured for $6 \mathrm{~h}$ at $37^{\circ} \mathrm{C}$ without decrease in viability, incubation with dyes at non-toxic concentrations at this temperature is a possible solution to 

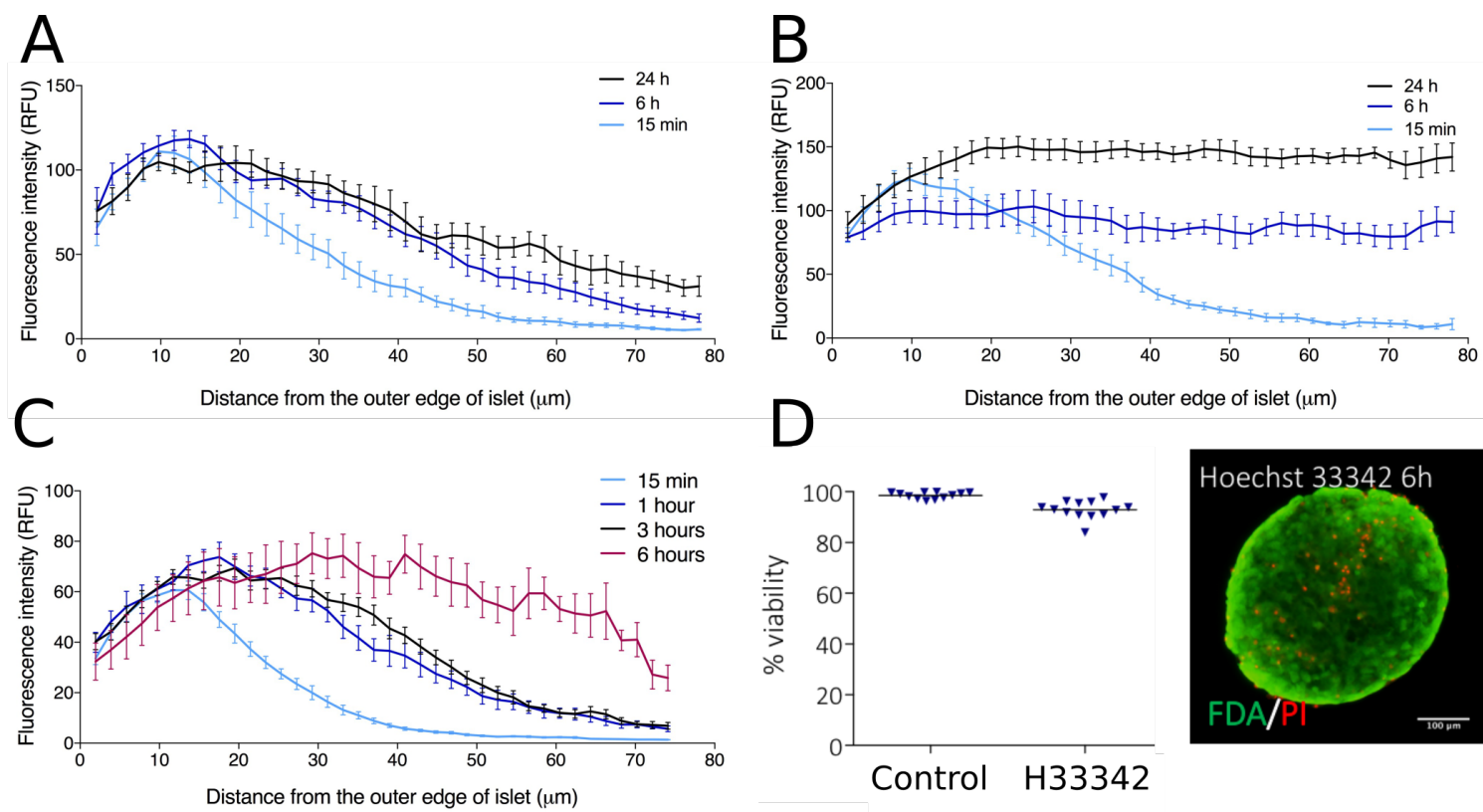

Fig. 3.8 Effect of temperature on diffusion of nuclear dye into pancreatic islets. (A) Staining profiles of pancreatic islets incubated Hoechst 33342 for $15 \mathrm{~min}, 6 \mathrm{~h}$ or $24 \mathrm{~h}$ at room temperature $(\mathrm{n}=6-7)$. (B) Staining profiles of pancreatic islets incubated Hoechst 33342 for $15 \mathrm{~min}, 6 \mathrm{~h}$ or $24 \mathrm{~h}$ at $37^{\circ} \mathrm{C}$ $(\mathrm{n}=5-7)$. (C) Staining profiles of pancreatic islets incubated Hoechst 33342 for $15 \mathrm{~min}, 1 \mathrm{~h}, 3 \mathrm{~h}$ or $6 \mathrm{~h}$ at $37^{\circ} \mathrm{C}(\mathrm{n}=7-15)$. (D) Viability of pancreatic islets incubated for $6 \mathrm{~h}$ at $37^{\circ} \mathrm{C}$ in culture medium (Control) or Hoechst 33342 staining solution (Hoechst). On the right, an example image of pancreatic islet incubated with Hoechst 33342 stained for viability at the end of the incubation. FDA = fluorescein diacetate; H33342 = Hoechst 33342; PI = propidium iodide. 
the diffusion problem. It will therefore be used for investigation of the imaging methods for islet viability assessment.

\subsubsection{Improvement of islet viability imaging method}

With the knowledge of the appropriate incubation time, imaging of islet viability was repeated using confocal microscopy. However, increasing the incubation time with viability dyes to $6 \mathrm{~h}$ did not bring a significant improvement to the quality of imaging of the islet core (Figure 3.9A). Even after $24 \mathrm{~h}$ of staining, it was not possible to detect the viability near the centre using confocal microscope (Figure 3.9B). The only fluorescent signal detectable near the centre of the cluster comes from PI, likely because red dyes, with their longer wavelengths which are not absorbed by cellular components, allow deeper penetration of light [175].

The next method investigated for imaging the viability of pancreatic islet in 3D was two-photon microscopy. This imaging was done in collaboration with Kevin O'Holleran and Martin Oliver Lenz from the Cambridge Advanced Imaging Centre. After $24 \mathrm{~h}$ staining, it was possible to image deeper cell layers than with confocal microscopy, but the staining in the centre of the islet still remained undetectable (Figure 3.9C).

Light sheet microscopy was investigated as another alternative imaging method in collaboration with Till Moreth and Francesco Pampaloni from Goethe University in Frankfurt. While the Hoechst and FDA staining still had very low resolution in the central part of the islet, it is possible to clearly image the presence of dead cells using PI (Figure 3.9D). Additionally, light sheet microscopy offers a possibility of multi-angle imaging which can further increase the resolution of the final 3D-reconstruction of the islet (Figure 3.9E). As shown in this Figure, it is possible for an islet to have a difference in viability of the periphery and the core.

To conclude, it was not possible to image the core of pancreatic islets using confocal or two-photon microscopy, likely because of the limited imaging depth. Pancreatic islets are densely packed clusters of cells, filled with insulin granules which highly scatter light [176]. These optical properties make islets particularly difficult to image, especially in the case of fresh tissue imaging (such as viability imaging) where procedures such as fixation and clearing are not available. Nevertheless, multi-angle light sheet microscopy seems to be able to provide a reliable image of the whole cell cluster viability, especially the presence of dead cells stained by propidium iodide. This method is not high-throughput and would not be suitable for clinical assessment of viability (due to time and equipment limitations) but it can be a useful tool for confirmation of viability in research settings (such as in efficacy of cryopreservation protocols). 

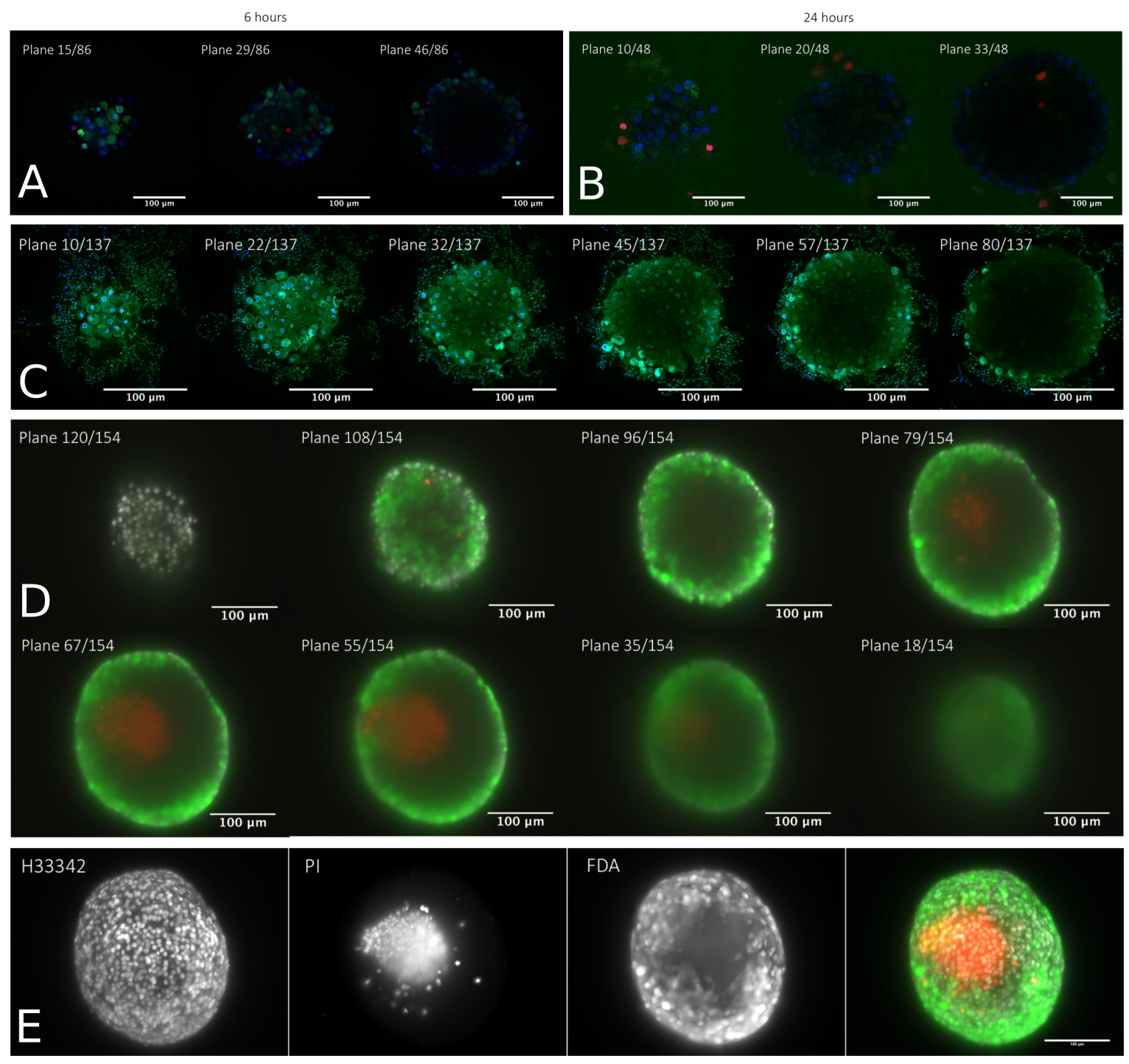

Fig. 3.9 Imaging of pancreatic islet core using confocal, two-photon and light sheet microscopy. (A) Pancreatic islet stained with FDA (green), PI (red) and Hoechst 33342 (blue) for $6 \mathrm{~h}$ at $37{ }^{\circ} \mathrm{C}$ imaged as a Z-stack on a confocal microscope. (B) Pancreatic islet stained with FDA (green), PI (red) and Hoechst 33342 (blue) for $24 \mathrm{~h}$ at $37^{\circ} \mathrm{C}$ imaged as a Z-stack on a confocal microscope. Long incubation time caused bleaching of FDA so the settings of the microscope was changed to detect the weak signal, resulting in high background signal. (C) Pancreatic islet stained with FDA (green) and Hoechst 33342 (white) for $6 \mathrm{~h}$ at $37{ }^{\circ} \mathrm{C}$ imaged as a Z-stack on a two-photon microscope. PI was not imaged using this method since the two-photon microscope was not equipped with the appropriate emission filter for PI wavelength. (D) Pancreatic islet stained with FDA (green), PI (red) and Hoechst 33342 (white) for $6 \mathrm{~h}$ at $37^{\circ} \mathrm{C}$ imaged as a Z-stack on a light sheet microscope. (E) 3D-reconstruction from multi-angle imaging (4 angles, $90^{\circ}$ apart) of pancreatic islet stained with FDA (green), PI (red) and Hoechst 33342 (white) on a light sheet microscope. The PI-stained core is likely to be so called hypoxic core: a result of impaired oxygen diffusion to the centre of large islets [174]. FDA = fluorescein diacetate H33342 = Hoechst 33342; PI = propidium iodide . 


\subsection{Discussion}

\subsubsection{Mononuclear cell viability assessment}

In Section 3.3, the adaptations to flow cytometric analysis for cryopreserved samples were outlined with regards to the cryobiology-specific needs of viability assessment method (Section 3.2).

The use of dual staining consisting of Syto13 and membrane-permeability dye has previously been suggested and validated for cryopreserved samples by Reardon et al. [88]. This chapter builds on this idea, by adding the steps of absolute count determination and comparison to pre-freeze samples. Moreover, the method was adapted to be able to determine post-thaw survival of cell subsets in mixed cell populations.

The method of assessment of absolute cell count of cell subsets has long been used for specific purposes - such as haematopoietic progenitor enumeration for transplantation where the kits of the enumeration of $\mathrm{CD} 4^{+}$cells are commercially available. However, these single-platform enumeration protocols (where counting beads are added directly to the stained cell sample) can only be performed in cases where the markers do not require washing steps. The method developed in this project, on the contrary, can be adapted for a variety of cell types and staining agents.

Many approaches for quantification of viability exist in the cryobiology field, many of which have significant shortfalls. Development of a standardised method and its adoption by the field would be very beneficial and would enable results from different laboratories/publications to be directly compared. The method suggested in this Chapter (reporting absolute number of cells both before and after freezing using flow cytometric analysis) could possibly be used for this purpose if validated and adopted by the cryobiology community.

\subsubsection{Pancreatic islet viability assessment}

\section{Limitations of current staining and imaging}

In Section 3.4, it was demonstrated that the current viability staining and imaging protocol ignores the viability of cells in the core of pancreatic islets. It is therefore not surprising that viability often does not correlate with function [92]. Figure 3.9E shows an example of an islet in which the viability in the periphery and the core is very different. This islet would, by current methods, be identified as a fully viable islet. Regarding the fact that central necrosis is a common phenomenon in large islets [174], this would suggest that viability of islet batches could be commonly overestimated. 
The optical properties of live islets are particularly challenging since the detection of fluorescent signals from the core of even average-sized $(100 \mu \mathrm{m})$ islets is very difficult or impossible using many available technologies. This may be related to the light-scattering insulin granules contained in $\beta$-cells [176]. The light sheet technology was the most successful in imaging the core of pancreatic islets, likely because it utilises multi-angle imaging.

The suggested staining and imaging method is suitable for research laboratories, but it is unfortunately not ideal for clinical use due to time limitations and equipment requirements. Other limitation of this work is the fact that identified diffusion properties are specific for Hoechst 33342 in aqueous solution. If exact diffusion rates of dyes and molecules are needed, the experiments would need to be repeated and include additional incubation times and replicates. Nevertheless, diffusion of other small molecules in aqueous solutions should, in theory, be quite similar and could be applied in design of new freezing protocols using non-penetrating cryoprotectants.

For future applications of these findings in staining or design of cryopreservation protocols, the variation in shape and size of islets should be taken into consideration: the ideal incubation time for each of them will vary. While it may be possible to sort islets by their size in laboratory experiments, it would be complicated in the clinical settings and therefore the optimal incubation time will be the one which works best for average sized islets, leaving small islets overexposed to the reagent and large islets not fully penetrated. Moreover, multicomponent diffusion may need to be taken into account in cryopreservation, especially when combining different cryoprotectants which may have different diffusion kinetics.

\section{Issues to be addressed in islet viability image analysis}

Achieving unbiased and precise image analysis of islets stained for viability constitutes another challenge. The current analysis comprises of manually classifying them into few groups. To enable objective quantification of viability of each individual islet and to interand intra-observer variation, the image analysis must be streamlined and automated.

Over the course of this project, thousands of images of islets stained with FDA and PI were taken on a wide-field microscope and analysed using a simple pixel intensity thresholding in ImageJ. Although the staining and microscope settings were identical within each experiment, significant variability in the fluorescent intensity between individual islets was identified. Taking into account the fact that the images were taken on a wide-field microscope, the likely reasons for this are the incomplete staining of all islets and variable size of the islets (small islets typically had higher fluorescent intensities than large ones because they would not flatten out easily under the pressure of a coverslip). 
A second issue identified was related to the fact that PI only stains cell nuclei whereas FDA stains the cytoplasm. Viabilities calculated using the positive areas therefore always overestimate the viability since the average size of a FDA-stained cell is larger than an average PI-stained nucleus. This fact is not necessarily an issue when comparing viabilities of different cryopreservation conditions but it could be another reason for the poor correlation between viability and functional assays.

Creation of a script in a suitable programming language (i.e. Python) which can be applied to quantify viability of islets of different sizes and fluorescent intensities and can be shared between labs and islet isolation centres would be the key step for standardising the analysis. To address the above issues, a suitable thresholding method must be identified and correction must be applied to address the cell size discrepancy between live and dead cells. While writing the macro code itself was not in the scope of this thesis, suggestions of the individual steps needed to achieve an unbiased, automated process are summarised below.

A simple thresholding protocol would include an analysis of the pixel intensity histogram for each colour channel, fitting a function onto the histogram and using the function parameters (such as centre $(\mathrm{C})$, error $(\boldsymbol{\delta})$, etc.), determining the position of the threshold (e.g. C-2 $\delta$ ). Area of the picture with the pixel intensity above this threshold would then be measured. The type of function (e.g. Gaussian, bimodal etc.) and the parameter which will be applied as a threshold will need to be carefully considered since the histogram shapes can vary significantly depending on the amount of staining in each colour channel relative to the background and spillover from the other channels.

For this reason, applying a conventional thresholding may not be sufficient even if the parameters of the imaging are kept the same within and between experiments. There is an option to employ adaptive thresholding (and/or machine learning) approach which would enable applying dynamic thresholds to different areas of one image, depending on the intensity of the surrounding pixels. This would also solve another problem which stems from the fact that using conventional viability staining method, the intensity of staining will not be the same throughout the islet volume.

The issue of the discrepancy between the FDA and PI area measurements could theoretically be solved by measuring the size distribution of individual FDA-stained and PI-stained cells and applying an appropriate correction to adjust for differences in positive areas of live and dead cells.

However, practically this approach would have several caveats. While the PI-stained nuclei are spaced out and can be easily counted to calculate an average size of PI-stained nucleus, with FDA-stained cytoplasm the borders of the cells are not always obvious and calculating area of these cells with currently existing tools will be complicated. Imaging on 
the wide-field microscope also means that several layers of cells can be seen in one image which makes this analysis almost impossible. Using confocal could make the image analysis easier but confocal microscope is not a standard piece of equipment in islet isolation centres. Nevertheless, applying this correction is not crucial for the accuracy of the measurement as long as it is acknowledged that the viability measured by this method is an overestimation of the actual viability.

\subsection{Conclusion}

Cryobiological assessment of sample viability after freezing and thawing presents special challenges compared to classical viability assessment in other fields. Although looking at cellular function provides more useful view of the cryopreserved sample quality, viability assessment has an important function in rapid screening of cryopreservation conditions and development of unbiased and precise methods is crucial.

A method of cryopreservation efficacy assessment for single cells which answers the specific needs of cryobiology was proposed in this Chapter and demonstrated on the example of mononuclear cells. In this method, two variables are recorded: the viability of the sample and the absolute cell count, which in combination provide the absolute number of viable cells. These variables should be recorded before freezing and after thawing for an accurate and robust evaluation of the efficiency of cryopreservation. This method is unbiased, reproducible and adaptable to specific characteristics of different cell populations.

The quality of the current viability assessment of pancreatic islets was investigated and it was demonstrated that the current staining protocol does not allow sufficient time for the dye diffusion throughout the cell cluster, resulting in the assessment of peripheral islet cells only. Approaches to accelerate diffusion were tested (including the use of DMSO as a penetration enhancer, increase in hydrostatic pressure and temperature). It was found that it can take up to $6 \mathrm{~h}$ at $37^{\circ} \mathrm{C}$ to achieve full staining by a nuclear dye. Furthermore, the imaging depth of confocal microscopy did not allow imaging of the islet core and therefore more powerful imaging methods were investigated. Multi-angle light sheet microscopy was identified as a suitable method for validation of successful cryopreservation protocols. 



\section{Chapter 4}

\section{Splenocyte cryopreservation with trehalose and permeabilising polymers}

\subsection{Introduction and aims}

Studies of DMSO-free cryopreservation methods for clinically-relevant cell types are limited. The only investigation of efficacy of trehalose without any penetrating cryoprotectants in single mononuclear cells showed decreased viability but increased clonogenic capacity compared to DMSO-cryopreserved cells [130]. Successful protocols which are free from DMSO or other penetrating cryoprotectants for cell clusters such as pancreatic islets or embryos have not been reported.

Realising the complexity of cell cluster cryopreservation, I opted to first use mononuclear cells to test the efficacy of trehalose and permeabilising polymers in cryopreservation. As single cells, mononuclear cells provide a simpler model for cryopreservation: it is not necessary to account for diffusion of cryoprotectants into and out of cell cluster or heat transfer. Cryopreservation of mononuclear cells (especially the progenitor fraction) is also used in treatment of patients and development of DMSO-free cryopreservation protocol would be potentially translatable to clinical practice. Their use was therefore preferred to the use of cell lines, whenever possible.

While haematopoietic progenitors already have use in clinical transplantation, therapies utilising $\mathrm{T}$ cells or B cells (such as regulatory $\mathrm{T}$ cells) are under development and their successful cryopreservation will be necessary. I therefore used the mixed population of mononuclear cells isolated from human spleen or bone marrow and analysed the survival of $\mathrm{CD}^{+} 4^{+}$haematopoietic progenitors, $\mathrm{CD}^{+} \mathrm{T}$ cells and $\mathrm{CD} 19^{+} \mathrm{B}$ cells. 
The first aim of this chapter was to determine the initial efficacy of trehalose alone and trehalose with PP-50 polymer using parameters chosen based on evidence in literature (200 mM trehalose and $100 \mu \mathrm{g} / \mathrm{mL}$ PP-50 concentrations). These initial results are described in Section 4.2.

The second aim was to investigate protocol parameters which were not specific to trehalose or polymers, to ensure that the work is done with the most efficient DMSO and trehalose protocols available. These parameters include cell density during cryopreservation, addition of fetal calf serum and variables involved in thawing protocol. Results are described in Section 4.3.

Using the optimised general protocol, I investigated the optimal times, temperatures and $\mathrm{pH}$ for incubation with trehalose and polymers in Section 4.4 and their optimal concentrations which balance efficacy and toxicity in Section 4.5. The development of a novel method of intracellular detection of trehalose by Raman spectroscopy is summarised in Section 4.6. The initial experiment using this method were then used to generate insights into the polymermediated trehalose uptake and safety of the new method.

\subsection{Initial efficacy of trehalose and PP-50}

In order to demonstrate the ability of the new cryopreservation method utilising trehalose and PP-50 polymer to preserve viable and functional mononuclear cells, singe cell suspensions generated from human spleen and bone marrow were cryopreserved in trehalose alone or trehalose with PP-50 and compared to DMSO controls. The overall post-thaw viability and percentages of cell subsets were determined by flow cytometry analysis. In addition to viability assessment, functionality of the $\mathrm{CD} 34^{+}$cell subset was determined using in vitro colony forming unit assays.

\subsubsection{Efficacy for cryopreservation of splenic mononuclear cells}

Splenic mononuclear cells from seven donors (Appendix A) were cryopreserved in three cryoprotectant combinations, all supplemented by FCS: DMSO, trehalose alone (T), trehalose with PP-50 (TP). The composition of cryoprotectant combinations used is shown in Table 4.1. Samples were cryopreserved at the cell density of $10^{7}$ cells $/ \mathrm{mL}$ and without any pre-freeze incubation time. Additionally, cells from one donor (D16) were cryopreserved with no cryoprotectant as a control. After thawing, the samples were stained with the Panel B1 and B2 antibodies (Appendix B) and analysed by flow cytometry to determine viability and phenotype. 
Table 4.1 Composition of cryoprotectant solutions

\begin{tabular}{ll}
\hline Code & Cryoprotectant solution composition \\
\hline DMSO & $10 \%$ DMSO + 10 \% FCS + DMEM \\
T & $200 \mathrm{mM}$ trehalose in DMEM + 10\% FCS \\
TP & $200 \mathrm{mM}$ trehalose in DMEM + 100 $\mathrm{g} / \mathrm{mL}$ PP-50 + 10 \% FCS \\
\hline
\end{tabular}

Notes: Codes used in this chapter and exact composition of each cryopreservation solution. DMSO = dimethyl sulphoxide, $\mathrm{T}=$ trehalose, $\mathrm{TP}=$ trehalose $+\mathrm{PP}-50$.

The percentage of doublets detected during flow cytometry analysis did not differ significantly between the three cryoprotectant conditions (data not shown), which suggests that there is no significant difference in adherence of cells cryopreserved in T, TP and DMSO. The percentages of alive nucleated cells and alive nucleated non-apoptotic cells were significantly lower after cryopreservation in T and TP compared to DMSO. Nevertheless, trehalose-cryopreserved samples had improved survival if compared to cells frozen without any cryoprotectant (Figures 4.1A and B). There is, therefore, a potential for the T and TP to be used for cryopreservation of human splenocytes.

There was no significant difference between the conditions in the frequency of CD34 ${ }^{+}$ haematopoietic progenitors in the population of alive nucleated cells (Figure 4.1D). There were three donors (D8, D12 and D14) with much higher percentage of CD34 ${ }^{+}$cells in all conditions (on average $0.312 \pm 0.075 \%$ of alive nucleated cells) and four donors with low percentage of $\mathrm{CD} 34^{+}$cells (on average $0.060 \pm 0.012 \%$ ). There we no significant differences in the donor demographics, suggesting that the differences cannot be explained by age, sex or type of the donors (DBD vs. DCD).

The percentages of CD19+ $\mathrm{B}$ cells were similar in DMSO, T and TP cryoprotectants (Figure 4.1E). However, significant difference was found between the frequency of $\mathrm{CD}^{+} \mathrm{T}$ cells after cryopreservation with DMSO and T or TP (Figure 4.1F). Lower survival in the trehalose, which generally did not provide as high protection as DMSO, would suggest that $\mathrm{T}$ cells may be more prone to damage caused by the freezing and thawing process compared to B cells. This may be a consequence of higher sensitivity of $\mathrm{T}$ cells to osmotic stress or dehydration but this hypothesis has yet to be tested.

Overall, no difference was found in cryoprotectant efficacy of trehalose alone and trehalose with PP-50. One potential reason for the failure of PP-50 to improve the cryopreservation outcome with trehalose is that the experimental design did not control for the $\mathrm{pH}$ of the freezing medium before addition to the cells. Permeabilising biopolymers are known to change their conformation in different $\mathrm{pH}$ and they were shown to improve trehalose loading only below the $\mathrm{pH}$ of 7.05 [141]. The medium used (DMEM) has a neutral $\mathrm{pH}$ of 7.4, which 

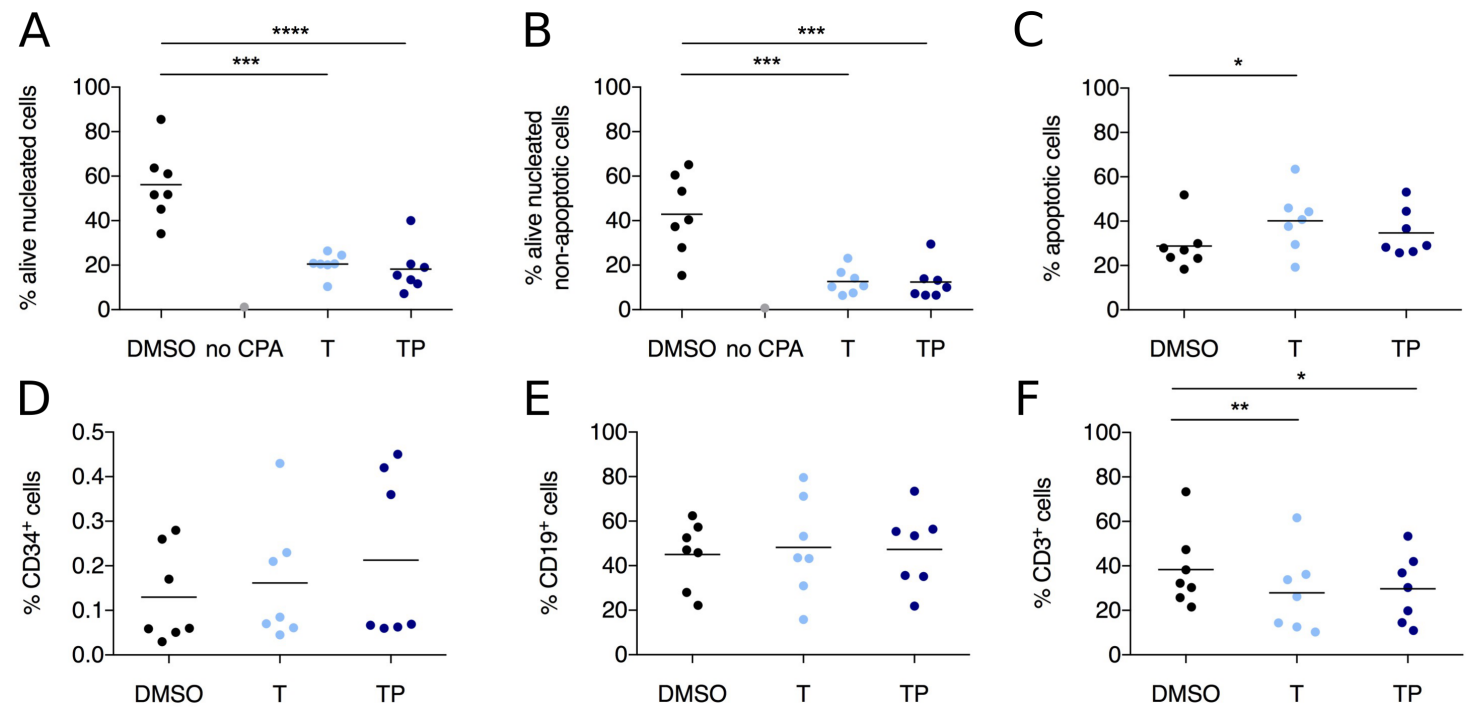

Fig. 4.1 Initial efficacy of DMSO, trehalose and PP-50 for cryopreservation of human mononuclear cells from spleen. (A) Percentage of alive nucleated cells (7-AAD ${ }^{-}$Syto $13^{+}$) out of all singlet events. (B) Percentage of alive nucleated non-apoptotic cells (7-AAD ${ }^{-}$Syto $13^{+}$annexin $\mathrm{V}^{-}$) out of all singlet events. (C) Percentage of apoptotic cells (7-AAD- Syto $13^{+}$annexin $\mathrm{V}^{+}$) out of alive nucleated cells. (D) Percentage of CD34 ${ }^{+}$progenitor cells out of alive nucleated cells. (E) Percentage of CD19 ${ }^{+} \mathrm{B}$ cells out of alive nucleated cells. (F) Percentage of $\mathrm{CD}^{+} \mathrm{T}$ cells out of alive nucleated cells. Mean of 7 experiments is shown by the line, individual values are presented as full circles. Differences between cryopreservation conditions were tested by one-way repeated measures ANOVA followed by Tukey's HSD test. Significant differences are marked by asterisks: $* \mathrm{p} \leq 0.05, * * \mathrm{p} \leq 0.01 ; * * * \mathrm{p} \leq 0.001$; $* * * * \mathrm{p} \leq 0.0001$. no $\mathrm{CPA}=$ no cryoprotectant; $\mathrm{T}=$ trehalose, $\mathrm{TP}=$ trehalose $+\mathrm{PP}-50$.

may be too high for efficient trehalose loading. Further experiments will be required to test this hypothesis, including the impact of low $\mathrm{pH}$ on cells.

\subsubsection{Efficacy for cryopreservation of bone marrow mononuclear cells}

Mononuclear cells from bone marrow of four deceased human organ donors (Appendix A) were cryopreserved using DMSO, T and TP cryoprotectant solutions (for composition, see Table 4.1). After thawing, cells were stained with Panel B1 and B2 antibodies (Appendix B) and analysed by flow cytometry.

The frequency of doublets did not significantly differ in the three cryopreservation conditions (data not shown). The percentage of alive nucleated cells and alive nucleated non-apoptotic cells was considerably lower in T and TP (Figure 4.2A and B). However, conversely to cryopreserved splenocytes, the level of apoptosis was significantly higher in TP cryopreserved bone marrow mononuclear cells (Figure 4.2C). 

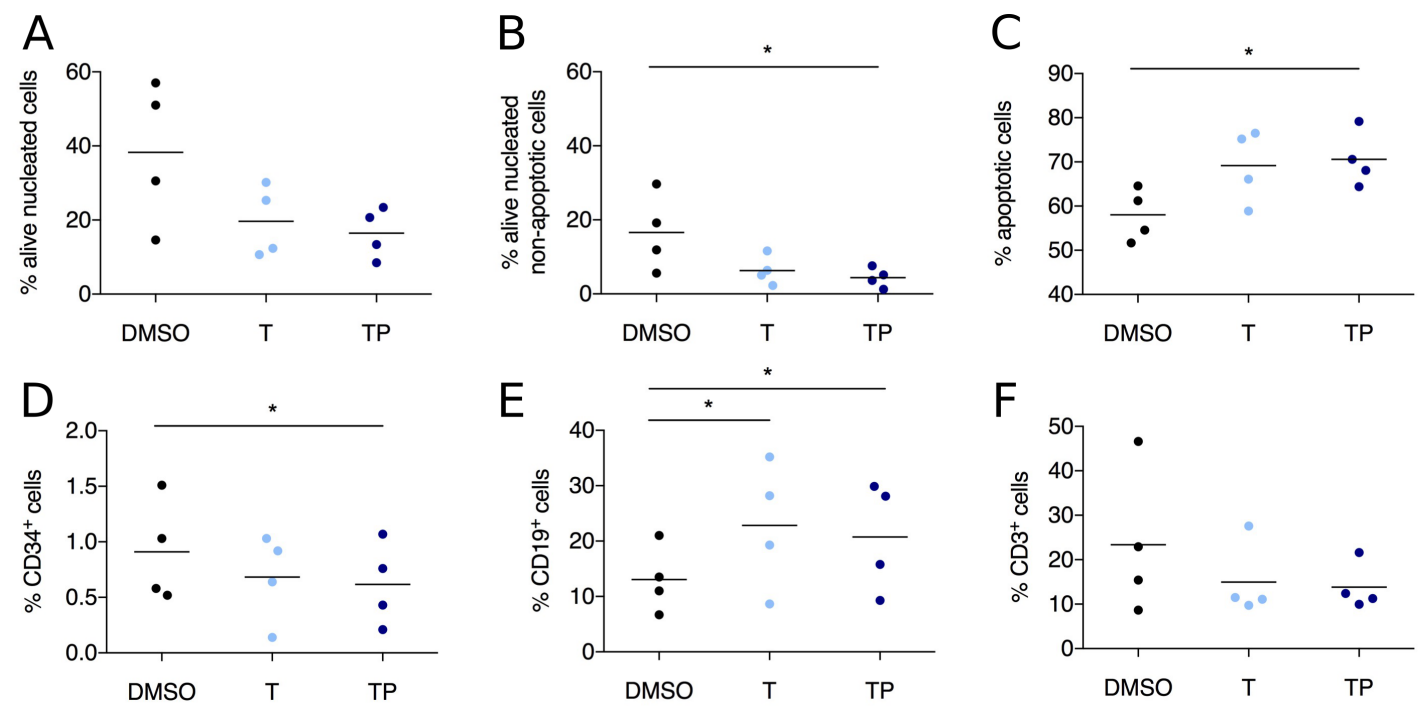

Fig. 4.2 Initial efficacy of DMSO, trehalose and PP-50 for cryopreservation of human mononuclear cells from bone marrow. (A) Percentage of alive nucleated cells (7-AAD ${ }^{-}$Syto $13^{+}$) out of all singlet events. (B) Percentage of alive nucleated non-apoptotic cells (7-AAD Syto $13^{+}$annexin $\mathrm{V}^{-}$) out of all singlet events. (C) Percentage of apoptotic cells (7-AAD Syto13 ${ }^{+}$annexin $\mathrm{V}^{+}$) out of alive nucleated cells. (D) Percentage of CD34 ${ }^{+}$progenitor cells out of alive nucleated cells. (E) Percentage of CD19 ${ }^{+}$ $\mathrm{B}$ cells out of alive nucleated cells. (F) Percentage of $\mathrm{CD}^{+} \mathrm{T}$ cells out of alive nucleated cells. Mean of 7 experiments is shown by the line, individual values are presented as full circles. Differences between cryopreservation conditions were tested by one-way repeated measures ANOVA followed by Tukey's HSD test. Significant differences are marked by an asterisk. $\mathrm{T}=$ trehalose, $\mathrm{TP}=$ trehalose + PP-50.

Significant difference was also found between the frequencies of $\mathrm{CD} 34^{+}$progenitors in DMSO and TP, even though the variation between the donors was high with percentages of $\mathrm{CD}^{+}{ }^{+}$cells ranging from $0.52 \%$ to $1.51 \%$ for DMSO-cryopreserved cells (Figure 4.2D).

As with cryopreserved splenocytes, there was a trend towards better survival of bone marrow $\mathrm{CD}^{+} \mathrm{T}$ cells in DMSO, although the difference was not statistically significant (Figure 4.2F). Conversely, CD19 ${ }^{+} \mathrm{B}$ cells survived significantly better in $\mathrm{T}$ and $\mathrm{TP}$ than in DMSO (Figure 4.2E). This was consistent with the hypothesis that B cells have lower sensitivity to the stress accompanying freezing than $\mathrm{T}$ cells.

There was no difference in efficacy of cryopreservation of bone marrow mononuclear cells in trehalose alone and trehalose supplemented with PP-50 (Figures 4.2A and B). This was also the case with splenocytes and suggests that mononuclear cells from these two sources behave similarly during the freezing and thawing procedure. 
A

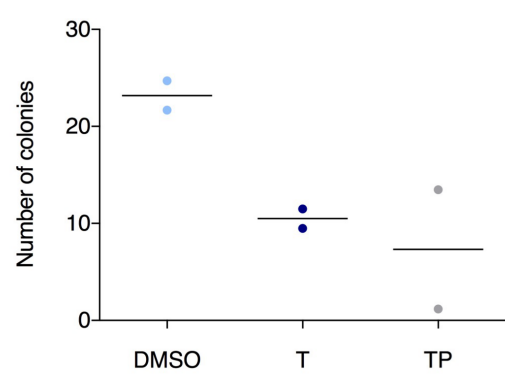

C

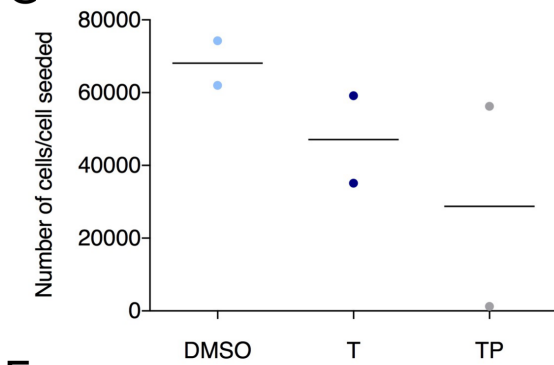

$\mathrm{E}$

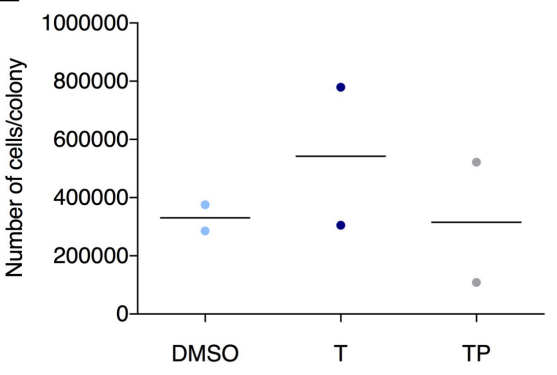

B

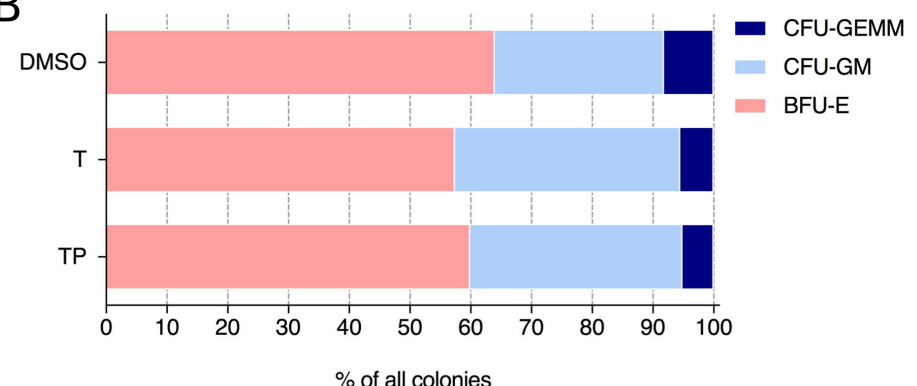

$\mathrm{D}$

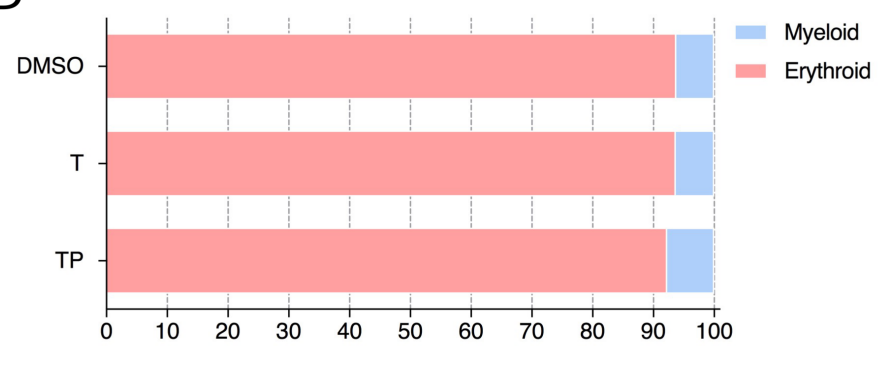

$\mathrm{F}$

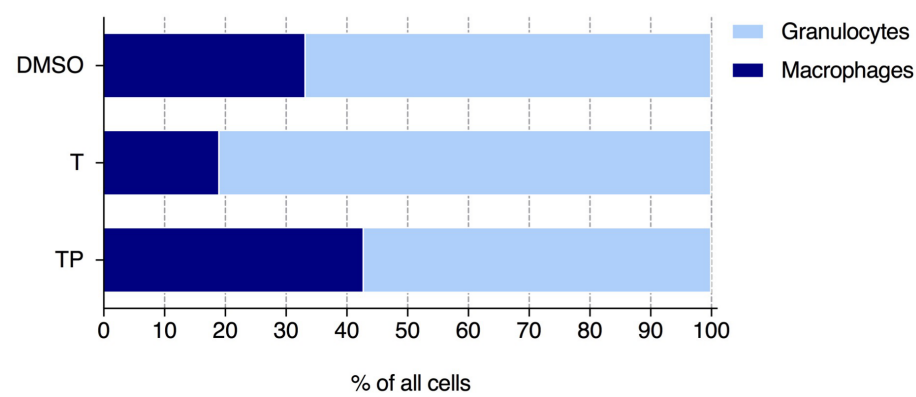

Fig. 4.3 Comparison of three cryopreservation conditions in 14-day haematopoietic CFU assay. (A) Total number of colonies grown at day 14 of culture per 100 CD $34^{+}$cells seeded. (B) Ratios of different colony types as a percentage of total colony number. (C) Average number of cells grown per $100 \mathrm{CD} 34^{+}$cells seeded determined by analysis in BD Trucount ${ }^{\mathrm{TM}}$ tubes. (D) Ratio of erythroid $\left(\mathrm{CD} 235 \mathrm{a}^{+}\right)$and myeloid $\left(\mathrm{CD} 33^{+}\right)$cells from all colonies determined by flow cytometry. (E) Average colony size, equivalent to average number of cells (determined by analysis in BD Trucount ${ }^{\mathrm{TM}}$ tubes) per colony. (F) Ratio of macrophages $\left(\mathrm{CD} 14^{+}\right)$and granulocytes $\left(\mathrm{CD} 15^{+}\right)$from all colonies determined by flow cytometry. In figures A, C and E, the mean of two experiments is shown by a line, individual values are presented as full circles. Statistical analysis was not performed due to a low sample size. $\mathrm{T}=$ trehalose, $\mathrm{TP}=$ trehalose $+\mathrm{PP}-50$. 


\subsubsection{Colony forming ability of cryopreserved $\mathrm{CD}^{+} 4^{+}$cells}

In addition to maintenance of viability, successfully cryopreserved CD34 ${ }^{+}$cells (haematopoietic progenitors) need to retain their function to be clinically useful. In vitro haematopoietic colony forming unit assays were performed to demonstrate that haematopoietic progenitors cryopreserved in trehalose or trehalose with PP-50 maintain their ability to proliferate and differentiate into different haematopoietic lineages.

Samples cryopreserved in DMSO, T and TP from two deceased human organ donors were thawed, the population of $\mathrm{CD} 34^{+}$cells was selected by FACS and CFU assays were performed. Between 700 and $1000 \mathrm{CD}^{+} 4^{+}$cells were sorted and seeded for each condition. Number of colonies after 14 days of culture were then related to number of cells seeded for each condition. Cell sorting and CFU preparation was performed with the help of Emily Calderbank from the Stem Cell Institute, University of Cambridge. Flow cytometry of the cells post-culture was performed to determine the percentages of erythroid cells (from the red and mixed colonies) and myeloid cells (from the white and mixed colonies), and as subsets of myeloid cells, granulocytes and macrophages. Analysis in BD Trucount ${ }^{\mathrm{TM}}$ tubes was performed to determine absolute numbers of cells grown during the assay.

The ability to form colonies of haematopoietic cells was decreased in T and TP conditions, compared to the DMSO control (Figure 4.3A). However, the distribution of colony types was similar for all three conditions, with approximately $60 \%$ of colonies of the BFU-E type, $33 \%$ CFU-GM and $6 \%$ CFU-GEMM (Figure 4.3B). Analysis of surface markers by flow cytometry confirmed that the ratio of erythroid and myeloid cells did not differ between these three conditions (Figure 4.3D) but there was a significant difference in percentages of macrophages and granulocytes between the conditions (Figure 4.3F). The proliferation potential of each seeded CD $34^{+}$progenitor was also higher in DMSO (Figure 4.3C) but the average colony size was greater for $\mathrm{T}$, although there was a considerable variability between the experiments (Figure 4.3E).

The data suggested that progenitors cryopreserved in each of these three cryopreservation solutions were capable of forming all haematopoietic lineages studied in this experiment (erythroid and myeloid cells, granulocytes and macrophages). However, the clonogenic efficiency of cells cryopreserved in trehalose or trehalose with PP-50 was decreased. This suggests cells cryopreserved in T or TP cryoprotectant solutions were more prone to suffer sublethal damage during cryopreservation. The possible mechanism could include decreased expression or loss of growth factor receptors resulting in unresponsiveness to growth factors driving the proliferation and differentiation of progenitors in CFU assay, as suggested for cryopreserved cells by Sasnoor et al. [177]. 


\subsection{General parameters of cryopreservation protocol}

Before further investigating the parameters specific to trehalose cryoprotectant, such as its concentration and variables involved with the pre-cryopreservation incubation period, general parameters of the trehalose cryopreservation protocol were optimised. These included cell density, addition of fetal calf serum and multiple thawing protocol parameters.

When optimising these general parameters for trehalose, an appropriate DMSO control was included in all experiments.

\subsubsection{Cell density}

For the purposes of clinical transplantation, it would be advantageous to cryopreserve cells in higher densities (over $10^{7}$ cells $/ \mathrm{mL}$ ). However, in research practice, cells are often cryopreserved in the concentration range of $10^{6}$ to $10^{7}$ cells $/ \mathrm{mL}$, mostly for practical purposes. I therefore investigated whether cell density has an impact on cell survival after cryopreservation with DMSO or alternative solutions containing trehalose and PP-50. The influence of difference cell densities has been previously studied for a narrow range of densities $\left(10^{8} \mathrm{vs}\right.$. $2 \times 10^{8}$ [178], $9 \times 10^{7}$ vs. $3 \times 10^{8}$ [64] or $2-5 \times 10^{7}$ vs. $2 \times 10^{8}$ cells $/ \mathrm{mL}$ [179] etc.), most of which did not detect any difference between the cell densities. In this project, the effect of cryopreservation of cells over a wider range of cell densities, from $10^{6}$ to $10^{9}$ cells $/ \mathrm{mL}$, was compared.

Table 4.2 Numbers of deceased human donor samples used for the cell density study

\begin{tabular}{lllll}
\hline & $10^{6}$ cell $/ \mathrm{mL}$ & $10^{7}$ cell/mL & $10^{8}$ cell $/ \mathrm{mL}$ & $10^{9}$ cell $/ \mathrm{mL}$ \\
\hline DMSO & $\mathrm{n}=3(\mathrm{D} 6, \mathrm{D} 8, \mathrm{D} 9)$ & $\mathrm{n}=3(\mathrm{D} 6, \mathrm{D} 8, \mathrm{D} 15)$ & $\mathrm{n}=4(\mathrm{D} 8, \mathrm{D} 9, \mathrm{D} 10, \mathrm{D} 15)$ & $\mathrm{n}=2(\mathrm{D} 8, \mathrm{D} 10)$ \\
$\mathrm{T}$ & $\mathrm{n}=3(\mathrm{D} 6, \mathrm{D} 8, \mathrm{D} 9)$ & $\mathrm{n}=3(\mathrm{D} 6, \mathrm{D} 8, \mathrm{D} 15)$ & $\mathrm{n}=4(\mathrm{D} 8, \mathrm{D} 9, \mathrm{D} 10, \mathrm{D} 15)$ & $\mathrm{n}=1(\mathrm{D} 10)$ \\
$\mathrm{TP}$ & $\mathrm{n}=3(\mathrm{D} 6, \mathrm{D} 8, \mathrm{D} 9)$ & $\mathrm{n}=3(\mathrm{D} 6, \mathrm{D} 8, \mathrm{D} 15)$ & $\mathrm{n}=4(\mathrm{D} 8, \mathrm{D} 9, \mathrm{D} 10, \mathrm{D} 15)$ & $\mathrm{n}=2(\mathrm{D} 8, \mathrm{D} 10)$ \\
\hline
\end{tabular}

Notes: For each condition characterised by the cryoprotectant used and the cell density, samples from $\mathrm{n}$ different donors were cryopreserved. In the brackets, the specific donor codes are indicated (demographics data can be found in Appendix A).

Samples were cryopreserved in three different cryoprotectants (DMSO, $200 \mathrm{mM}$ trehalose (T), $200 \mathrm{mM}$ trehalose $+50 \mu \mathrm{g} / \mathrm{mL}$ PP-50 (TP) at four different cell densities $\left(10^{6}, 10^{7}, 10^{8}\right.$ or $10^{9}$ cells $/ \mathrm{mL}$ ). In total, splenocytes from five deceased human organ donors were dedicated for this set of experiments. However, due to the limitation of the low total cell yield per spleen, it was not possible to cryopreserve samples in all cell densities from every donor (Table 4.2). After thawing (following the un-optimised thawing protocol, Section 4.3.3), 

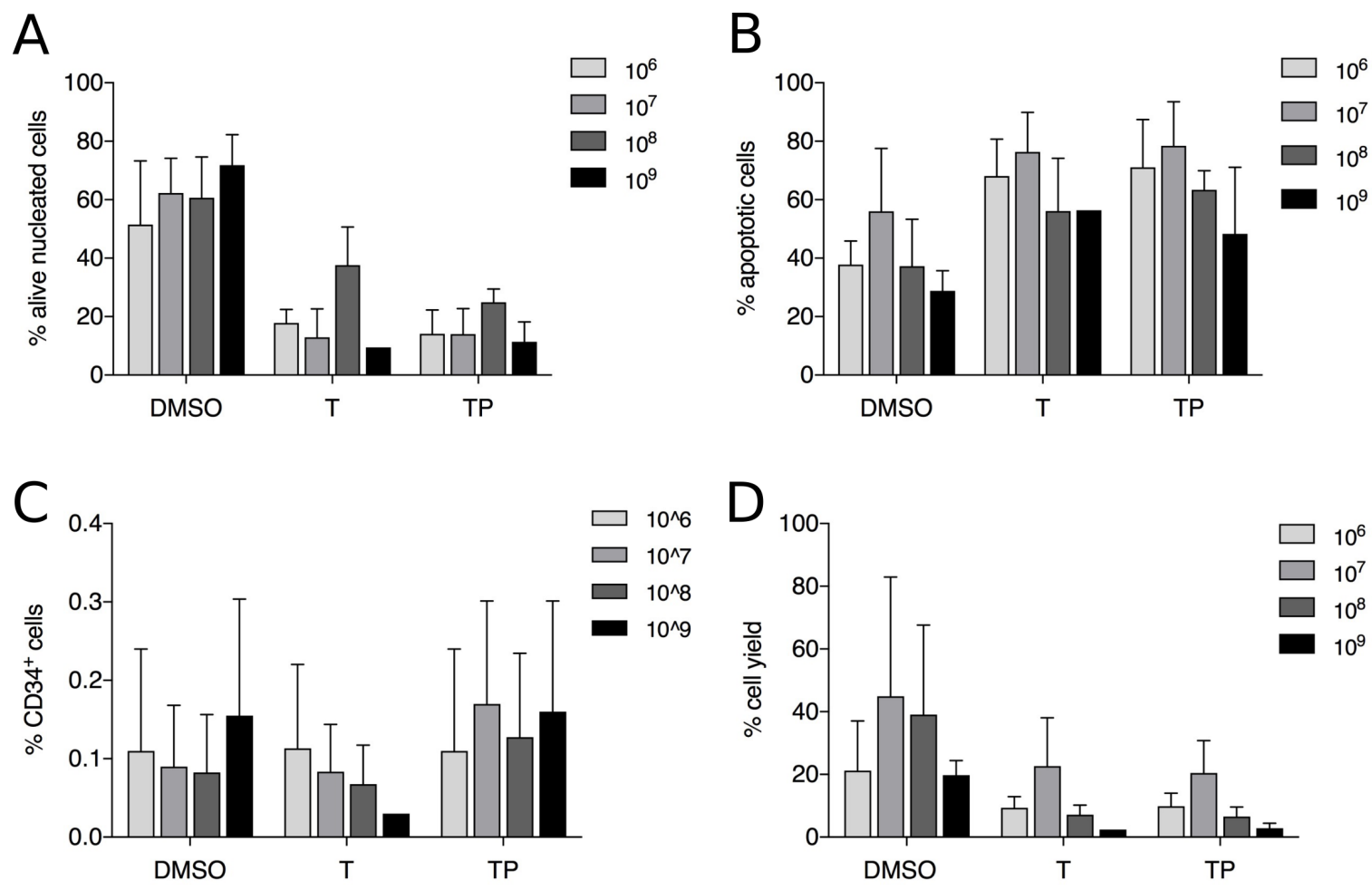

Fig. 4.4 Effects of different cell densities on the outcome of cryopreservation with DMSO, trehalose and trehalose + PP-50. (A) Percentage of alive nucleated cells (eFluor780- Syto13 ${ }^{+}$) out of all singlet events. (B) Percentage of apoptotic cells (eFluor780- Syto13 ${ }^{+}$annexin $\mathrm{V}^{+}$) out of alive nucleated cells. (C) Percentage of CD34 ${ }^{+}$cells of alive nucleated cells. (D) Post-thaw cell yield expressed as a percentage of cell count after thawing out of pre-freeze cell count. All bars represent mean $\pm \mathrm{SD}$, differences between cell densities of each cryoprotectant were tested by two-way mixed model ANOVA followed by Tukey's HSD test, no significant differences were found. $\mathrm{T}=$ trehalose, $\mathrm{TP}=$ trehalose $+\mathrm{PP}-50$

the absolute cell count was determined using the haemocytometer and viability and surface markers were analysed by flow cytometry (using panels A1 and A2 described in Appendix B).

For the control DMSO condition, higher cell densities resulted in better cell survival (Figure 4.4A) and lower apoptosis (Figure 4.4B). For T and TP, the impact of cell density on cell survival was less clear, although a cell density of $10^{8}$ cells $/ \mathrm{mL}$ appeared to be most effective for both conditions (Figures 4.4A and B). There was no difference in survival of $\mathrm{CD}_{3} 4^{+}$progenitors in different cell concentrations for any of the three cryoprotectants (Figure 4.4C).

Lowest absolute cell losses (determined by post-thaw haemocytometer count) were detected for the $10^{7}$ cell/mL concentration in all three cryoprotectants (Figure 4.4D). Possible reasons for the decrease in absolute cell count after thawing include freezing-related cell losses and losses during the two washing steps in the thawing procedure. For concentrations 
of $10^{8}$ cells $/ \mathrm{mL}$ or higher, cell clumping represents a major cause of cell losses; large clumps were visible at $10^{8}$ cells $/ \mathrm{mL}$ cell density for T and TP and in $10^{9}$ cells $/ \mathrm{mL}$ cell density for all the conditions. The reason for decreased yield at the $10^{6}$ cells $/ \mathrm{mL}$ concentration is less clear. One hypothesis is that in lower cell densities, the volume of extracellular fluid is larger which has an impact on dynamics of the freezing process. The extracellular space has a different composition and generally freezes much earlier than the cytoplasm [36]. The cells at low concentrations do not effectively limit ice crystal growth in the extracellular space (during both freezing and thawing), leading to increased damage to the cells. Theoretically, toxicity of the cryoprotectant could also play a role as more molecules of DMSO or trehalose can act on fewer cells but this would be less likely with a non-toxic cryoprotectant such as trehalose.

In conclusion, the highest tested cell density resulted in the best cell survival in DMSO. However, in the $10^{9}$ cells $/ \mathrm{mL}$ concentration, the cells tended to clump which caused significant cell losses. The ideal cell concentration for DMSO would therefore be between $10^{8}$ and $10^{9}$ cells $/ \mathrm{mL}$. For trehalose (conditions T and TP), the cell clumping started to occur at the $10^{8}$ cells $/ \mathrm{mL}$ density. Therefore, for these conditions, the ideal cell density would be lower than for DMSO, likely between $10^{7}$ and $10^{8}$ cells $/ \mathrm{mL}$. Unless the clumping can be prevented by additives (such as DNase, anticoagulants such as acid-citrate- dextrose and heparin or hydroxyethyl starch [180]), cryopreservation in higher densities than those stated seems to be impractical.

\subsubsection{Addition of fetal calf serum}

Addition of fetal calf serum (FCS) into the cryopreservation medium was previously shown to have a beneficial effect on cell recovery of bone marrow cells from mouse and human [52, 181]. Addition of serum is thought to protect the cells both during the freezing process and subsequent post-thaw processing when the marrow cells are fragile [181]. The effect of supplementation of cryopreservation media with FCS on cell yield and recovery of mononuclear cells from human spleen was evaluated in this Section.

Samples of splenic mononuclear cells from three deceased human donors were cryopreserved at $10^{7}$ cells $/ \mathrm{mL}$ using DMSO or $200 \mathrm{mM}$ trehalose supplemented with different amounts of FCS (0\%, $10 \%, 50 \%$ or $100 \%)$. After thawing, cells were stained for flow cytometry using antibody panels A1 and A2 (Appendix B) and absolute cell count was determined using Trucount beads.

Addition of FCS to DMSO cryopreservation medium did not affect the post-thaw percentages of cryopreserved $\mathrm{CD} 34^{+}, \mathrm{CD} 19^{+}$or $\mathrm{CD} 3^{+}$subsets in the samples (Figure 4.5A and B). However, slightly lower percentage of alive nucleated cells could be found after thawing in sample without FCS and with $10 \%$ or more FCS added (not statistically significant, 

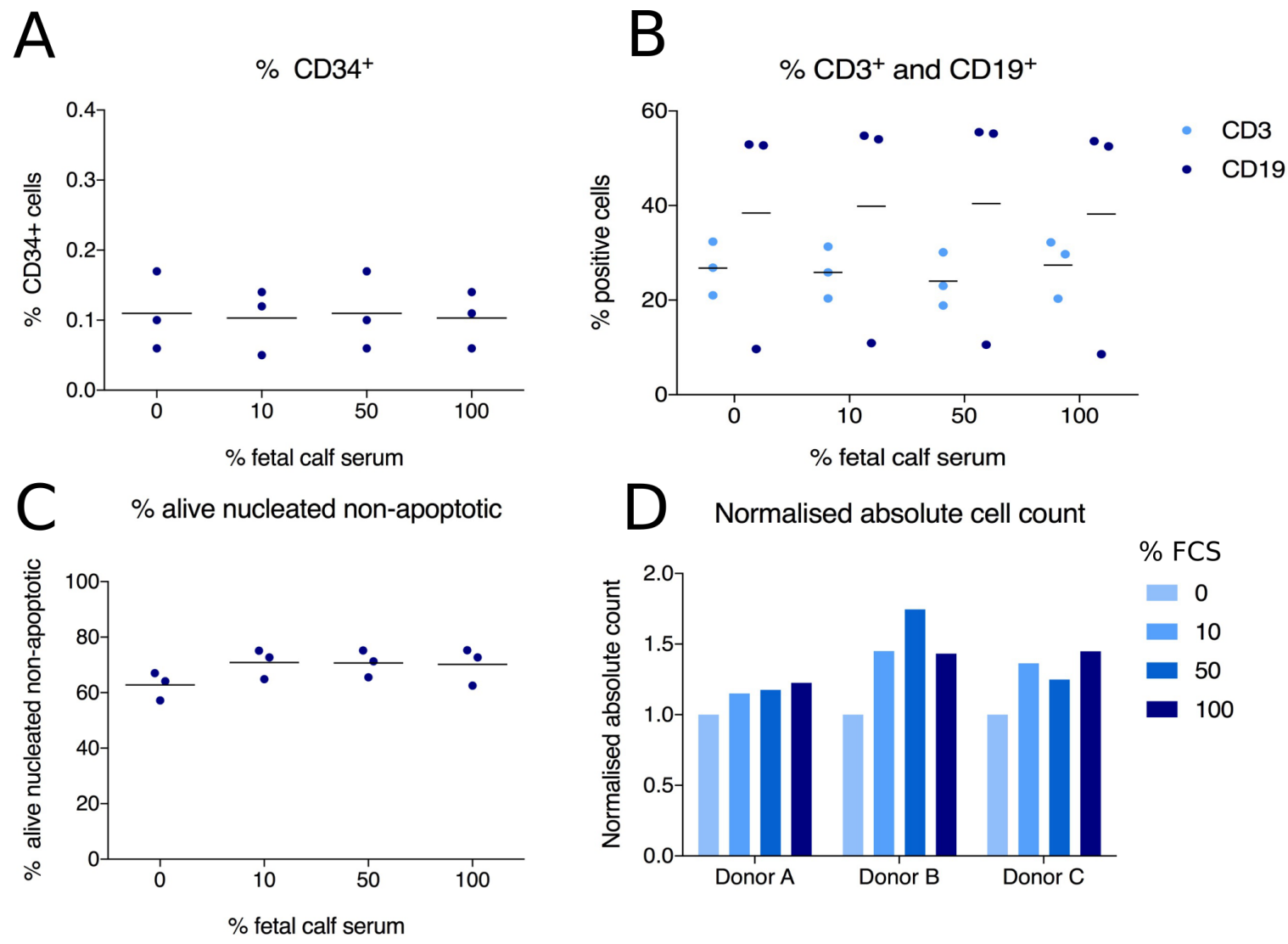

Fig. 4.5 Effects of addition of fetal calf serum to DMSO-cryopreserved splenocytes. (A) Post-thaw percentage of $\mathrm{CD} 4^{+}$cells of alive nucleated cells. (B) Post-thaw percentages of $\mathrm{CD}^{+}$and $\mathrm{CD} 19^{+}$ cells of alive nucleated cells. (C) Post-thaw percentage of alive nucleated non-apoptotic cells (7-AAD ${ }^{-}$ Syto13 ${ }^{+}$annexin $\mathrm{V}^{-}$) out of all singlet events. (D) Post-thaw absolute number of alive nucleated cells $\left(7-\mathrm{AAD}^{-}\right.$Syto $13^{+}$) normalised to the absolute number of alive nucleated cells in $0 \%$ FCS for each donor. Mean of three experiments is shown as a line, individual values are presented as full circles. Differences between conditions were tested by one-way repeated measures ANOVA followed by Tukey's HSD test, no significant differences were found. 
A
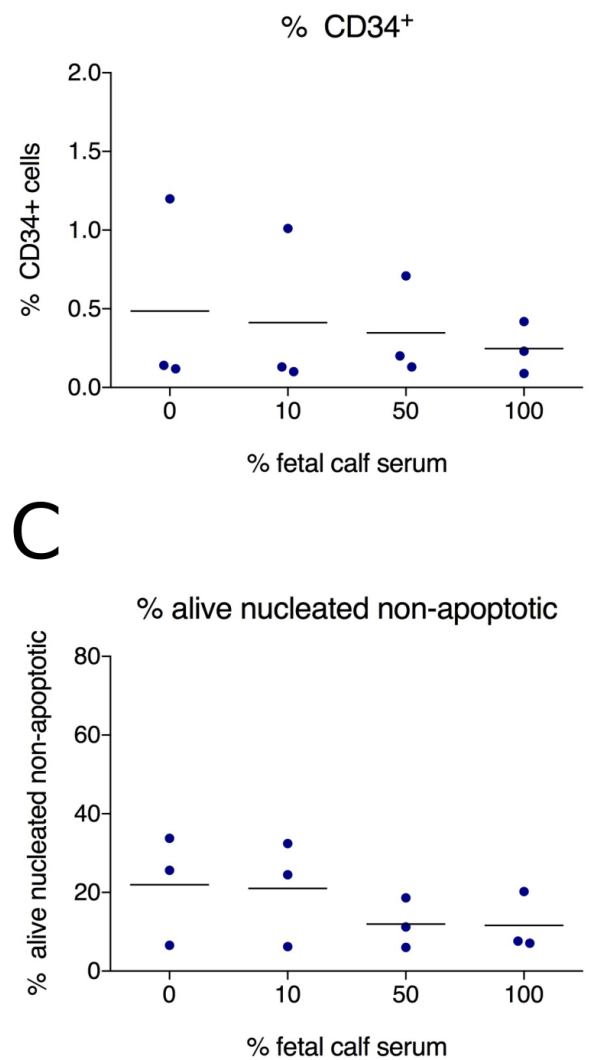

B

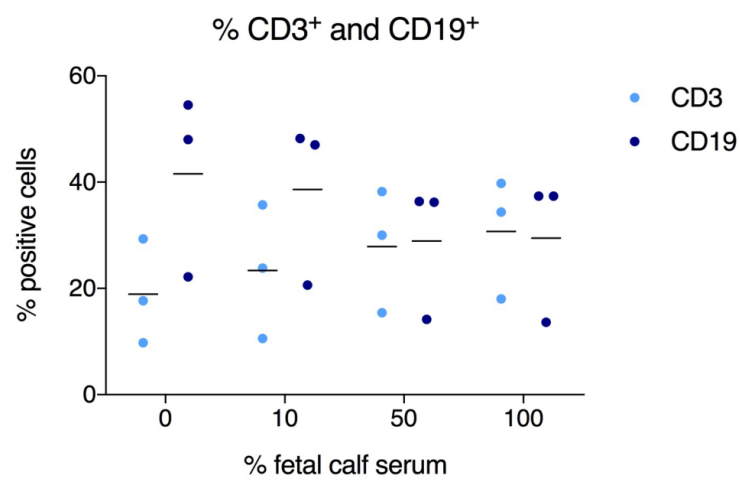

$\mathrm{D}$

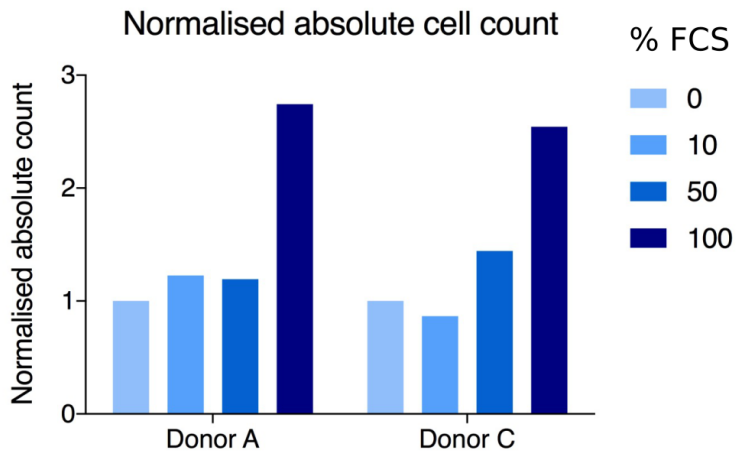

Fig. 4.6 Effects of addition of fetal calf serum to trehalose-cryopreserved splenocytes. (A) Post-thaw percentage of $\mathrm{CD}_{3} 4^{+}$cells of alive nucleated cells. (B) Post-thaw percentages of $\mathrm{CD}^{+}$and $\mathrm{CD} 19^{+}$ cells of alive nucleated cells. (C) Post-thaw percentage of alive nucleated non-apoptotic cells (7-AAD Syto $13^{+}$annexin $\mathrm{V}^{-}$) out of all singlet events. (D) Post-thaw absolute number of alive nucleated cells $\left(7-\mathrm{AAD}^{-}\right.$Syto $13^{+}$) normalised to the absolute number of alive nucleated cells in $0 \%$ FCS for each donor. Mean of three experiments is shown as a line, individual values are presented as full circles. In (D), individual values of two experiments are displayed (data is missing for one donor due to technical error). Differences between conditions were tested by one-way repeated measures ANOVA followed by Tukey's HSD test, no significant differences were found. 
Figure 4.5C). There was also a trend in the absolute number of alive nucleated cells: all samples with added FCS (regardless of concentration) had higher numbers of cells than samples without FCS (Figure 4.5D).

In trehalose-cryopreserved samples, the overall viability was much lower, as expected. Percentage of surviving CD $34^{+}$cells did not significantly differ between the different FCS concentrations but there was a trend towards higher proportion of surviving CD34 ${ }^{+}$cells in samples will less FCS added (Figure 4.6A). The same trend was observed for CD19+ cells while the opposite was true for $\mathrm{CD}^{+}$cells (Figure 4.6B). Regarding total number of alive nucleated non-apoptotic cells, large variability was observed and no clear trend was distinguishable (Figure 4.6C). However, the absolute number of surviving cells in $200 \mathrm{mM}$ trehalose in $100 \%$ FCS was more than double the other conditions (Figure 4.6D).

The addition of FCS to the DMSO-based medium showed small but significant difference in the percentage of surviving cells and a trend towards higher absolute numbers. This is in agreement with previous studies although different assays were used there (mainly cell yield and CFU assays) $[52,181]$. Since FCS is known to exhibit certain amount of cryoprotective effect, its addition has had a large effect on the success of cryopreservation with trehalose (as seen in Figure 4.6D). It would be reasonable to postulate that this effect may be somehow diminished if the cryopreservation efficacy of this condition is increased (possibly to a level similar to the DMSO-cryopreserved samples seen in Figure 4.5D). The trend towards better survival of $\mathrm{CD} 34^{+}$and $\mathrm{CD} 19^{+}$in harsher conditions has been observed across all the experiments with splenocytes.

To conclude, although the addition of FCS had a limited effect in successful cryopreservation conditions, in subsequent experiments, all cryopreservation media were supplemented with FCS based on the evidence from these experiments and previous publications. Since the positive effect of FCS addition was not found to be concentration-dependent in the DMSO-cryopreserved samples, $10 \%$ FCS was used in all subsequent cryopreservation experiments. There is some evidence that using homologous serum instead of fetal calf serum is advantageous [181] and the substitution of FCS for a GMP-compatible alternative would be necessary before clinical translation. However, due to the unavailability of homologous serum and cost of GMP-compatible alternatives, FCS was used for subsequent experiments.

\subsubsection{Optimisation of thawing protocol}

Thawing protocols introduce many variables into the cryopreservation procedure which may impact outcomes. These variables include: thawing rate, thawing time, composition of the thawing medium, temperature and $\mathrm{pH}$ of the thawing medium, technique of addition of the thawing medium, the final dilution of the cryoprotectant, method of cell manipulation 
(centrifugation speed, pipetting), and number of washes. I therefore investigated the effects of some of these variables on post-thaw viability and apoptosis of human splenocytes. The parameters examined were:

- thawing time (until the last ice crystal visible or 10 minutes)

- temperature of the thawing medium (room temperature or $37^{\circ} \mathrm{C}$ )

- technique of cryoprotectant addition (quickly or drop-wise)

- number of washes (one or two).

Rapid thawing in $37{ }^{\circ} \mathrm{C}$ water bath is recommended in most protocols. Removing the sample from the water bath as soon as the ice has thawed is also thought to prevent the damage caused by DMSO in higher temperatures [106]. It is recommended that thawing medium should then be added slowly to reduce the osmotic shock to the cells [69]. The number of washing steps varies in different laboratories; some prefer no washing steps in order to eliminate any cell losses, while others wash the cells twice to thoroughly remove the cryoprotectant. The standard protocol used in many laboratories involves rapid thawing in $37{ }^{\circ} \mathrm{C}$ water bath until the last crystal is visible, drop-wise addition of thawing medium at room temperature (DMEM + 50\% FCS) up to ten times the original volume (for $1 \mathrm{~mL}$ of sample it is diluted to $10 \mathrm{~mL}$ ) and two washing steps for removal of the cryoprotectant (Figure 4.7A).

Samples from three donors were cryopreserved in DMSO and different three donors in TP (Appendix A). All samples were cryopreserved at density of $10^{7}$ cells $/ \mathrm{mL}$ with no incubation time. This study was designed with two arms: one for DMSO-cryopreserved samples and one for trehalose cryopreserved samples. It was assumed that the optimal thawing protocol for trehalose and trehalose with PP-50 will be similar and therefore the samples were cryopreserved using trehalose and PP-50. Moreover, because of low cell yields from the thawed TP samples, only four different thawing protocols were investigated for each thawed sample, compared to eight different protocols from the DMSO samples. The design of this study is outlined in Figure 4.7.

After thawing the samples, cells were counted using haemocytometer, stained with Panel $\mathrm{C}$ antibodies (Appendix B) and analysed by flow cytometry. Comparisons of combinations of thawing protocols which were used to inform decisions about the final optimised protocol are shown for each arm of this study. Separate optimal protocols were then derived for DMSO-cryopreserved samples and trehalose-cryopreserved samples. 


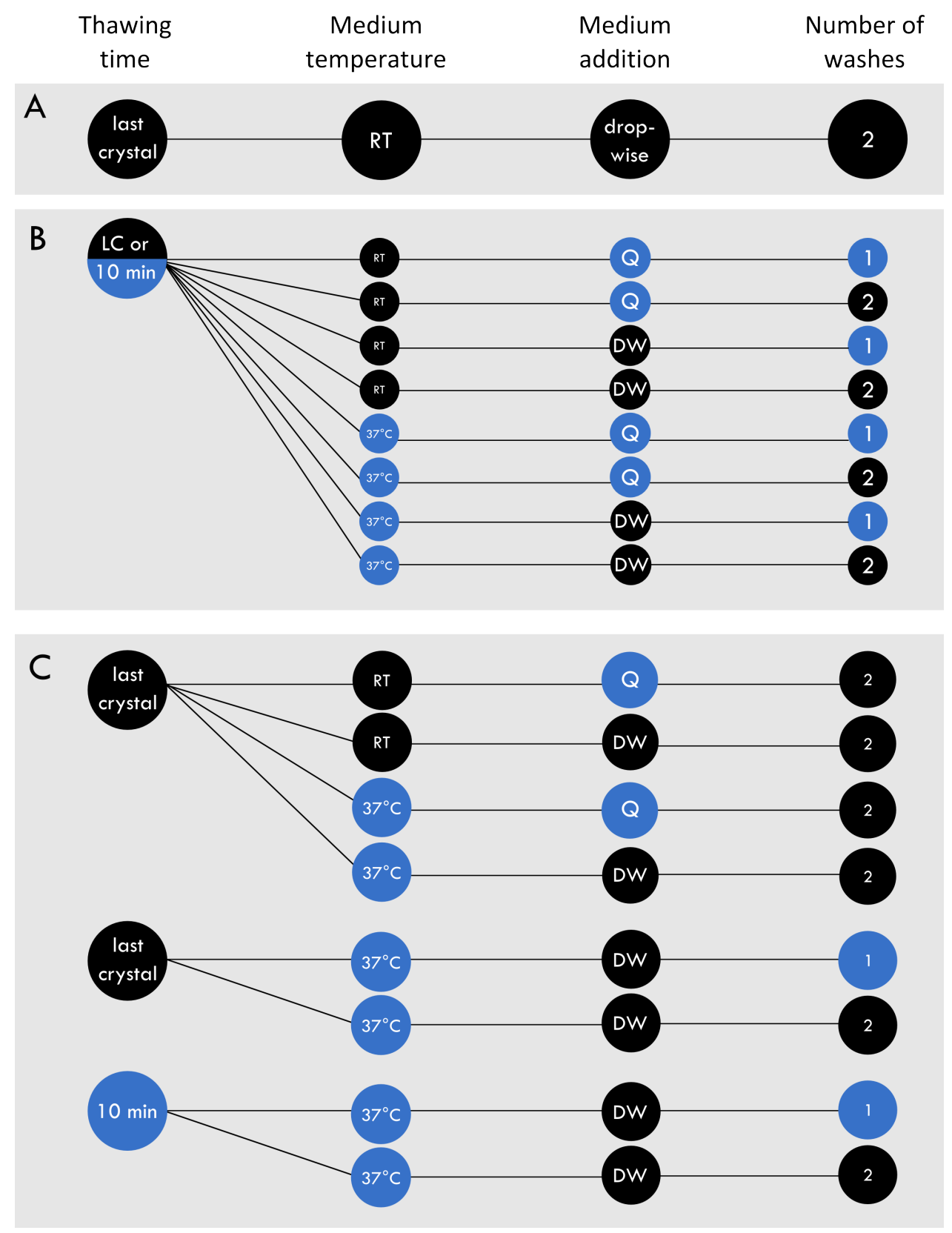

Fig. 4.7 Study design of the thawing protocol refinement for DMSO and TP (trehalose + PP-50) samples. (A) Current un-optimised protocol for thawing mononuclear cells from spleen, bone marrow and peripheral blood. (B) Study design for DMSO samples. First sample was thawed until the last crystal was left, second one for $10 \mathrm{~min}$. Each sample was then split into 8 tubes for 8 different protocols as outlined. Blue circles mark derivation from the currently used protocol. (C) Study design for TP samples. First sample was thawed until the last ice crystal was left, split into four tubes and used for decision about the medium temperature and medium addition. Based on this decision, the medium temperature was fixed to $37^{\circ} \mathrm{C}$ and medium addition technique to drop-wise in the following experiments. Two samples were thawed, one until the last crystal left and one for $10 \mathrm{~min}$, each was split into two tubes and thawed following the outlined protocols. $\mathrm{RT}=$ room temperature; $\mathrm{LC}=$ last crystal; $\mathrm{Q}=$ quickly; DW = drop-wise 


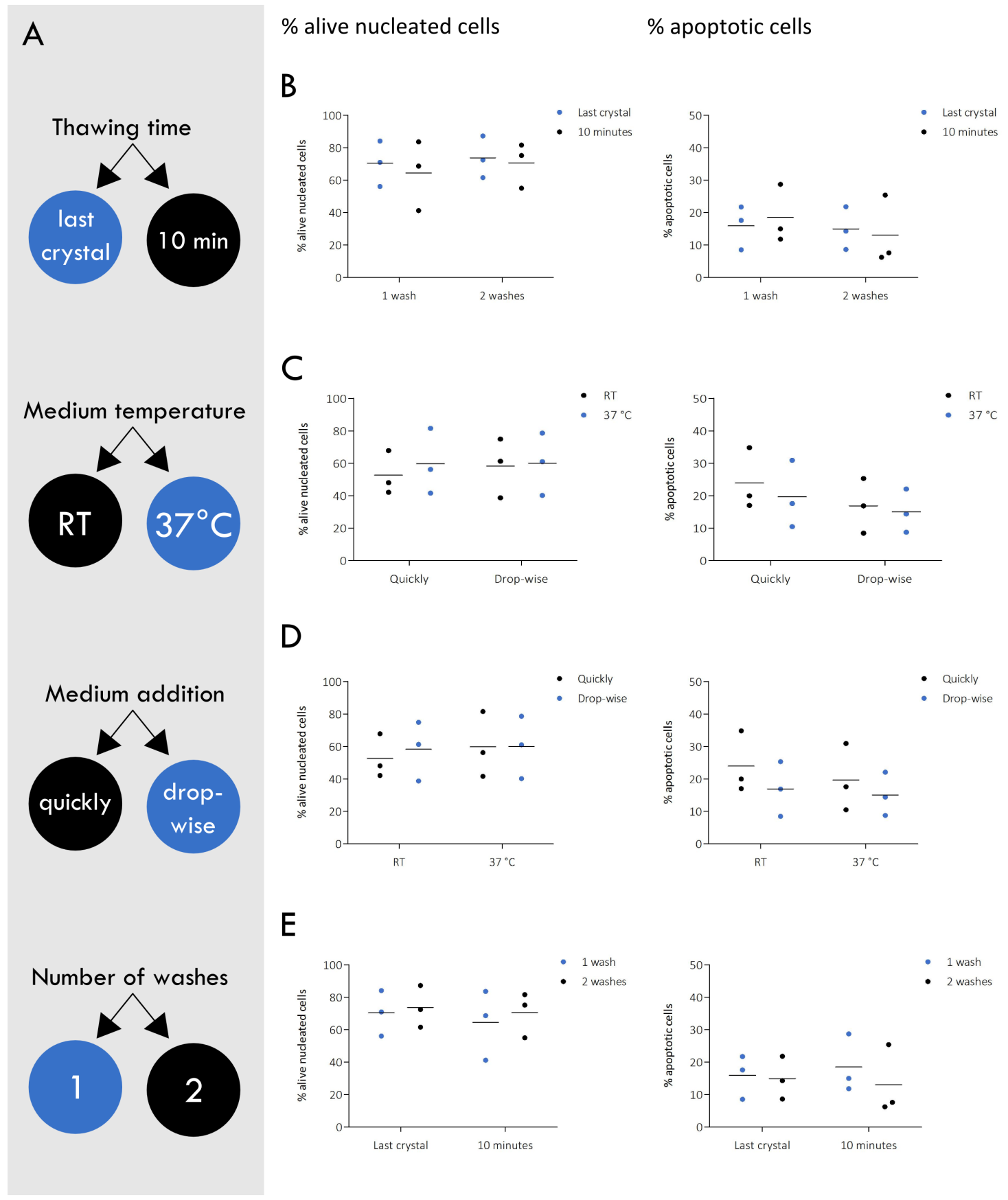

Fig. 4.8 Effect of thawing protocol changes on cryopreservation with DMSO. (A) Schematic of the decision points in the thawing protocol, the optimal option for each variable is shown in blue circle. (B) Percentage of alive nucleated cells (7-AAD $\mathrm{Ayto}^{-} 3^{+}$) of all singlet events for selected protocol combinations which served as a basis for the decision. (C) Percentage of apoptotic cells (7-AAD ${ }^{-}$ Syto $13^{+}$annexin $\mathrm{V}^{+}$) of alive nucleated cells for selected protocol combinations which served as a basis for the decision. Means of three experiments (circles) are shown by the horizontal lines. No significant differences were found after testing with two-way ANOVA with Tukey's HSD test. RT = room temperature. 

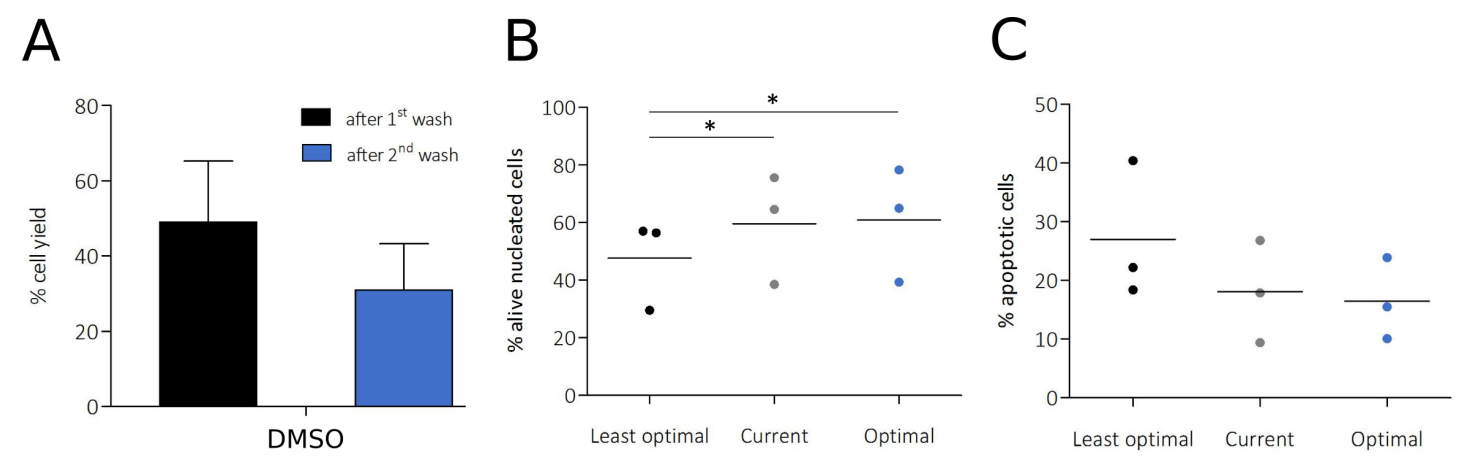

Fig. 4.9 Final comparison of thawing protocols for DMSO-cryopreserved samples. (A) Cell yield after first and second wash showed as a percentage of the original cell count before freezing. This data was derived from the results in cell density study in the Section 4.3.1. (B) Percentage of alive nucleated cells $\left(7-\mathrm{AAD}^{-}\right.$Syto $\left.13^{+}\right)$of all singlet events for the least optimal protocol (10 min, room temperature, quick addition, 1 wash), currently used protocol (last crystal, room temperature, drop-wise addition, 2 washes) and the optimal protocol (last crystal, $37^{\circ} \mathrm{C}$, drop-wise addition, 2 washes). (C) Percentage of apoptotic cells (7-AAD Syto $13^{+}$annexin $\mathrm{V}^{+}$) of alive nucleated cells for the least optimal protocol, currently used protocol and the optimal protocol. Bars in (A) represent mean $\pm \mathrm{SD}$, difference was tested by paired t-test. Means of three experiments in (B) and (C) (circles) are shown by the horizontal lines differences between the protocols in (B) and (C) were tested by one-way repeated measures ANOVA followed by Tukey's HSD test. Significance $(p \leq 0.05)$ is marked by an asterisk.

\section{Thawing procedure for DMSO-cryopreserved samples}

To inform decisions about the four variables in the thawing protocol described earlier, I compared percentages of alive nucleated cells and percentage of apoptotic cells from the 16 thawing protocols (mean data from three experiments). Only selected protocols are shown in Figure 4.8 for clarity but the result was derived from comparison of all protocols tested. The variability between samples was high and no significant differences were found after analysis by two-way ANOVA with Bonferroni post-hoc test. Nonetheless, trends detected by the comparison of all the protocols are noted and described, as even small improvement caused by changing an individual variable can potentially contribute to greater efficacy of the final optimised protocol.

Of the two thawing durations tested, thawing at $37{ }^{\circ} \mathrm{C}$ until the last crystal is visible showed a trend towards better mean viability for all the conditions tested, compared to thawing for $10 \mathrm{~min}$ (Figure 4.8B). There was no difference in the level of apoptosis. Thawing medium temperature of $37^{\circ} \mathrm{C}$ appeared to be superior, with lower percentage of apoptotic cells for all conditions and higher viability, especially for samples with the quick addition of the thawing medium (Figure 4.8C). Human splenocytes appeared to be adversely affected by quick addition as it made them sensitive to every change in the protocol. In contrast, when 
using drop-wise addition of medium, the viability and apoptosis remained generally stable in all 8 protocols (Figure 4.8D).

Washing the cells twice (rather than once) resulted in overall higher quality of the thawed cells (Figure 4.8E) but it also led to decreased absolute cell count, although the difference was not statistically significant. As a part of the cell density study (Section 4.3.1), a cell count both after first and second wash was performed to determine the average cell loss caused by the washing. The decrease in cell yield between the first and second wash was on average $18.8 \pm 9.1 \%$ (Figure 4.9A). The decrease in viability of the cells that have been only washed once is likely caused by toxic effects of the remaining DMSO in the system. In the optimised protocol, I therefore opted to wash the cells once but with increased volume of the thawing medium to achieve higher dilution of DMSO.

The recommended optimal protocol, which was used in all subsequent experiments, consisted of thawing until the last crystal is visible, drop-wise dilution of the cryopreserved sample to $20 \times$ of the original sample volume by thawing medium (DMEM + $50 \%$ FCS) warmed up to $37{ }^{\circ} \mathrm{C}$, followed by one centrifugation and washing step (Figure 4.8A). The protocol is similar to that routinely used in many laboratories, with the exception of medium temperature, and does not result in significant changes in viability and apoptosis. Compared to the least optimal protocol (thawing for $10 \mathrm{~min}$, quick addition of medium at room temperature, 1 wash), the most successful protocol resulted in a statistically significant increase in viability $(13.2 \%)$ and $10.5 \%$ decrease in apoptosis (Figures 4.9B and C).

\section{Thawing procedure for trehalose-cryopreserved samples}

For TP samples, it was not possible to split each sample into eight tubes to test all 16 protocols simultaneously due to low post-thaw cell count of individual cryopreserved samples. Instead, using each sample, I was able to compare four different protocols (Figure 4.7C). In the first set of experiments, all four protocols included thawing until the last crystal left and two washes. The decision about medium temperature and technique of medium addition was based on this first set of experiments.

There was no significant difference between the two techniques of thawing medium addition but similarly to the DMSO samples in previous section, the quick addition of the medium appeared to make the cells more susceptible to changes in thawing procedure (Figure 4.10D). This suggests that this effect is not dependent on the type of cryoprotectant and is likely the result of the osmotic shock that cells undergo during quick removal of the cryoprotectant. I therefore opted for drop-wise addition of the thawing medium in the optimised protocol. No obvious trend was apparent when comparing the two medium 


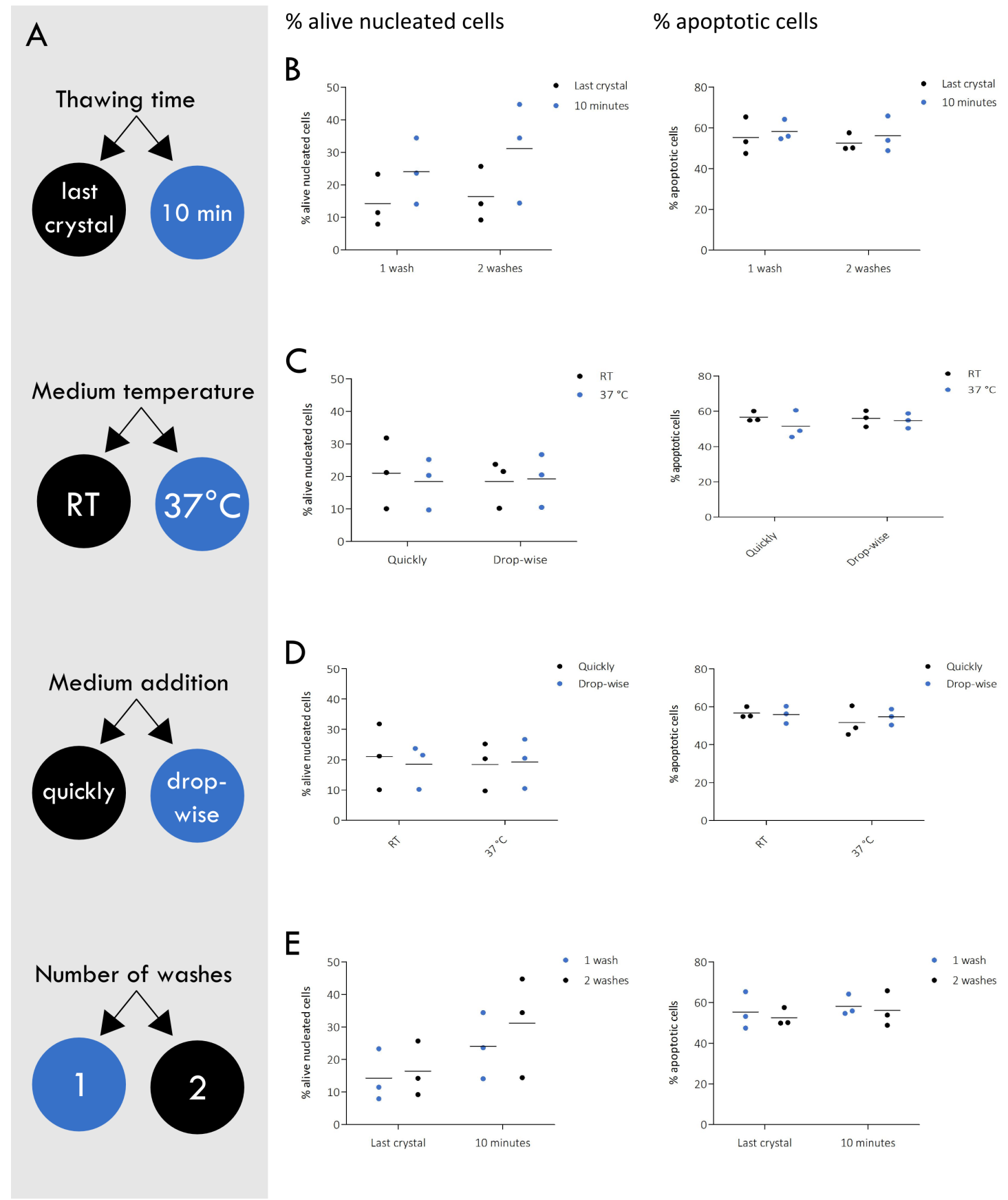

Fig. 4.10 Effect of thawing protocol changes on cryopreservation with trehalose + PP-50. (A) Schematic of the decision points in the thawing protocol, the optimal option for each variable is shown in blue circle. (B) Percentage of alive nucleated cells (7-AAD ${ }^{-}$Syto $13^{+}$) of all singlet events for selected protocol combinations which served as a basis for the decision. (C) Percentage of apoptotic cells (7-AAD- Syto $13^{+}$annexin $\mathrm{V}^{+}$) of alive nucleated cells for selected protocol combinations which served as a basis for the decision. Means of three experiments (circles) are shown by the horizontal lines. No significant differences were found after testing with two-way ANOVA with Tukey's HSD test. RT = room temperature. 
A

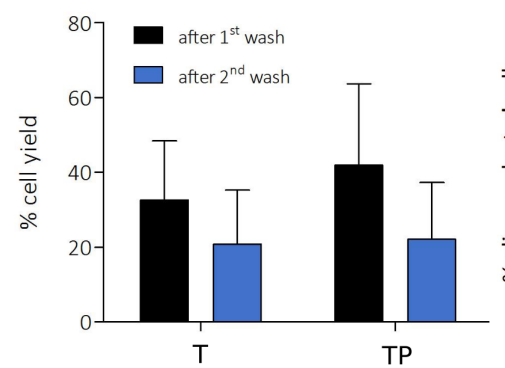

B

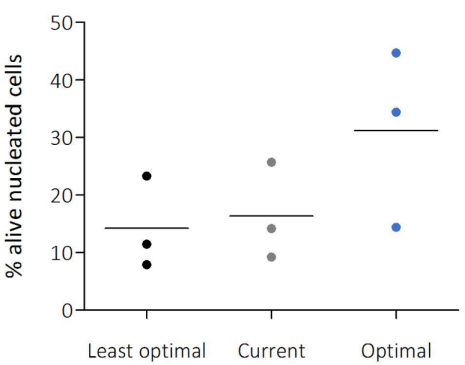

C

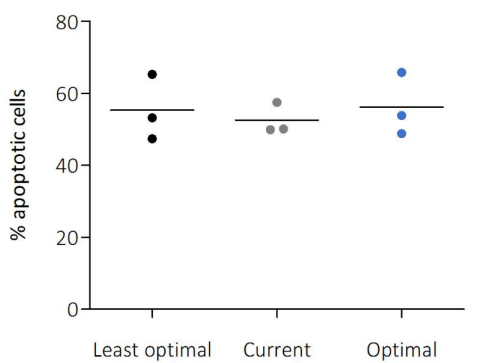

Fig. 4.11 Final comparison of thawing protocols for TP-cryopreserved samples. (A) Cell yields after first and second wash showed as a percentage of the original cell count before freezing. This data was derived from the results in cell density study in the Section 4.3.1. (B) Percentage of alive

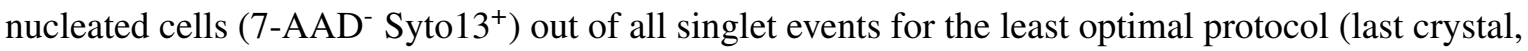
$37^{\circ} \mathrm{C}$, drop-wise addition, 1 wash), currently used protocol (last crystal, room temperature, drop-wise addition, 2 washes) and the optimal protocol ( 10 minutes, $37^{\circ} \mathrm{C}$, drop-wise addition, 2 washes). (C) Percentage of apoptotic cells $\left(7-\mathrm{AAD}^{-}\right.$Syto13 ${ }^{+}$annexin $\mathrm{V}^{+}$) out of alive nucleated cells for the least optimal protocol, currently used protocol and the optimal protocol. Bars in (A) represent mean $\pm \mathrm{SD}$, difference was tested by paired t-test. Means of three experiments in (B) and (C) (full circles) are shown by the horizontal lines; differences between the protocols in (B) and (C) were tested by repeated measures ANOVA followed by Tukey's HSD test. No significant differences were found.

temperatures (Figure 4.10C). Therefore, $37{ }^{\circ} \mathrm{C}$ thawing medium was used in the optimised protocol, similar to the DMSO thawing protocol.

After confirming the $37^{\circ} \mathrm{C}$ thawing medium and drop-wise addition steps, two additional samples were thawed from each donor, one until the last crystal was left and one for $10 \mathrm{~min}$. Two protocols were performed with each sample, varying in number of washes (Figure 4.7C).

The thawing time appeared to have the biggest influence the outcomes of the thawing procedure in the case of trehalose-cryopreserved splenocytes. Unlike DMSO samples, trehalose-cryopreserved human splenocytes showed higher viability if the thawing time in the $37{ }^{\circ} \mathrm{C}$ water bath was prolonged to $10 \mathrm{~min}$, although this was not statistically significant (Figure 4.10B). It is possible that the short period of culture before the cryoprotectant removal allows more time for equilibration of cells with the now unfrozen extracellular solution, which ameliorates the subsequent osmotic shock caused by the dilution of the cryoprotectant. Since trehalose (unlike DMSO) does not exert toxic effects on cells at this concentration, the post-thaw culture period could be extended to maximise beneficial effect.

The choice of the optimal number of washes follows the same reasoning as for the DMSO-cryopreserved samples. Cell losses caused by an extra wash appeared to be greater than the small increase in cell viability. Analysis of data from the cell density experiments 
(Section 4.3.1) indicates that two washes result in an additional $11.8 \pm 7.5 \%$ and $19.8 \pm 8.5 \%$ cell loss in for T and TP cryopreservation solutions respectively (Figure 4.11A).

In conclusion, the final optimised protocol for TP differs from the ideal thawing protocol for DMSO-cryopreserved cells only in the thawing time (Figure 4.10A). No changes in the apoptosis level could be observed between the least optimal, currently used and the optimal protocol derived by this set of experiments (Figure 4.11C). However, the recovery of the cells markedly increased in the optimised protocol. The thawing protocol resulted in up to $16.9 \%$ improvement in the viability of the TP cryopreserved splenocytes (Figure 4.11B). While the previously used standard protocol was close to optimal for DMSO-cryopreserved samples (Figures 4.9B and C), it resulted in inferior cryopreservation outcomes for trehalosecryopreserved samples.

\subsection{Incubation parameters for trehalose and PP-50}

\subsubsection{Incubation time at $37^{\circ} \mathrm{C}$}

Trehalose does not freely permeate through the cellular membrane with the exception of small amount of passive diffusion. Increasing the incubation time with trehalose before freezing could therefore enable sufficient time for trehalose to diffuse into the intracellular space and thus increase its protective effect. Another approach is to facilitate loading of trehalose using different approaches which were summarised in the Introduction section 1.9.3. In this thesis, the approach explored was by using permeabilising polymers (PP-50 and PP-75) which were shown to increase trehalose loading into ovine erythrocytes as a function of incubation time [141, 182].

Human splenocytes were cryopreserved using three cryoprotectant conditions: DMSO ( 0 h or $1 \mathrm{~h}$ incubation), T and TP conditions ( $0 \mathrm{~h}, 1 \mathrm{~h}, 2 \mathrm{~h}$ or $3 \mathrm{~h}$ incubation). Flow cytometry of the fresh samples was performed (Antibody panels A1 and A2) and all samples were cryopreserved at $10^{7}$ cells $/ \mathrm{mL}$. After addition of the cryoprotectant, samples were either transferred immediately to the $-80{ }^{\circ} \mathrm{C}$ freezer in a freezing container $(0 \mathrm{~h})$ or incubated in water bath set to $37{ }^{\circ} \mathrm{C}$ for the specified time and then frozen. After thawing, using the optimised cryopreservation procedure (Section 4.3.3), samples were analysed using Antibody panels B1 and B2 (Appendix B) and absolute count determined by counting beads.

The results confirm that incubation with DMSO has a detrimental effect on cells: it decreased viability, cell yield after cryopreservation and increased apoptosis (Figures 4.12A, $\mathrm{B}$ and $\mathrm{C}$ ). Because this result confirmed the expected toxic effect, incubation with DMSO was not pursued in the following replicates of the experiment (hence $n=1$ for DMSO $1 \mathrm{~h}$ ). 


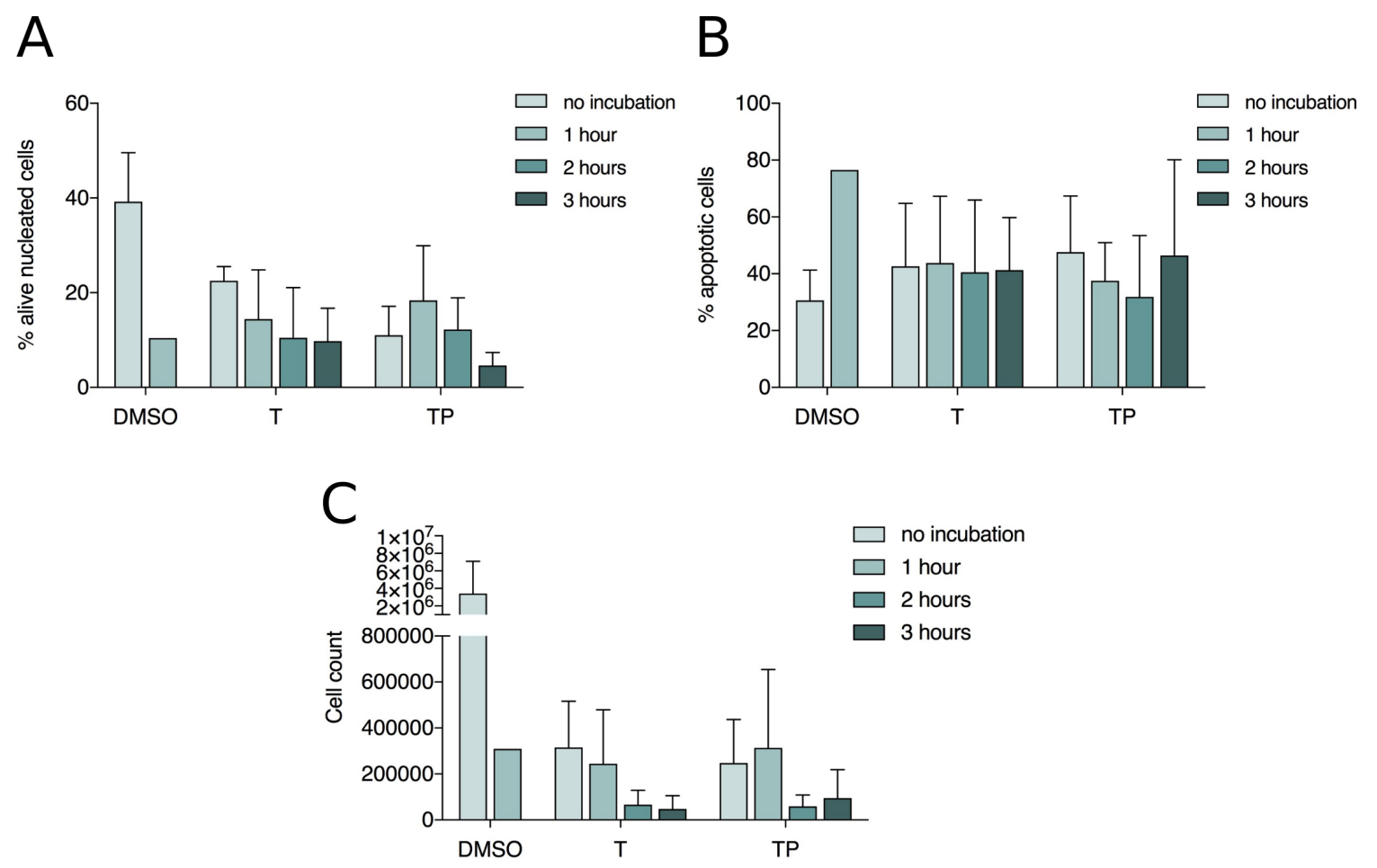

Fig. 4.12 Optimisation of length of incubation at $37^{\circ} \mathrm{C}$ before splenocyte cryopreservation (A) Percentage of alive nucleated cells (7-AAD ${ }^{-}$Syto $13^{+}$) out of all singlet events. (B) Percentage of apoptotic

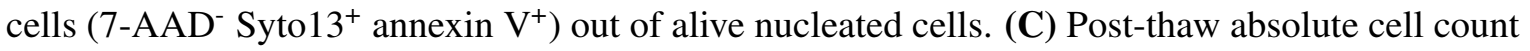
determined by flow cytometry analysis with BD Trucount ${ }^{\mathrm{TM}}$ tubes. All bars represent mean $\pm \mathrm{SD}$ $(\mathrm{n}=3$, except for $\mathrm{n}=1$ for DMSO $1 \mathrm{~h})$. Differences between different incubation times were tested by two-way ANOVA followed by Tukey's HSD test for T and TP sets of conditions. No significant differences were found. $\mathrm{T}=$ trehalose, $\mathrm{TP}=$ trehalose $+\mathrm{PP}-50$. 
A trend of decrease of viability and absolute cell count with increasing incubation time was observed with trehalose alone (although much slower compared to DMSO)(Figure 4.12A and C) and the apoptosis level did not change over the studied period (Figure 4.12B).

Increased incubation time did not result in a statistically significant improvement in viability. In both $\mathrm{T}$ and $\mathrm{TP}$ conditions, trehalose would enter the intracellular space of cells very slowly by passive diffusion. This should theoretically lead to improvement of cryoprotection. The reason of the decrease of post-thaw cell yield and viability observed in these experiments could hypothetically be due to the non-ideal culture conditions during incubation. In other words, any beneficial cryoprotective effect of longer incubation with trehalose would be masked by the rate of cell death during the incubation. The rates of cell death in culture was therefore investigated in Section 4.4.2.

In conclusion, the pre-freezing incubation period at $37{ }^{\circ} \mathrm{C}$ with cryoprotectant is not beneficial for DMSO or trehalose alone but for trehalose supplemented with PP-50 polymer, one-hour incubation results in slight improvement in post-thaw cell yield and cell survival. Adjusting the culture conditions during incubation could further increase the potential of including an incubation period before cryopreservation.

\subsubsection{Incubation temperature}

In the previous section it was shown that pre-freeze incubation does not improve cryopreservation with trehalose. Since some of the cell death may occur during the incubation period due to non-ideal culture conditions (rather than during the cryopreservation), a series of experiments were conducted to optimise the temperature during the pre-cryopreservation incubation period.

\section{Culture with $200 \mathrm{mM}$ trehalose at different temperatures}

In the first set of experiments, the rate of cell death in culture at $4{ }^{\circ} \mathrm{C}, 20^{\circ} \mathrm{C}$ and $37{ }^{\circ} \mathrm{C}$ in $200 \mathrm{mM}$ trehalose was investigated over the course of $3 \mathrm{~h}$ (as described in the Methods section 2.5.3). Absolute numbers of alive nucleated cells were measured at five time points for each temperature $(0,30,60,120$ and $180 \mathrm{~min})$.

This confirmed that incubation at $37{ }^{\circ} \mathrm{C}$ with $200 \mathrm{mM}$ trehalose causes detrimental effects to the splenocytes, decreasing the absolute cell count to $79.4 \pm 5.7 \%$ of the original cell number after three hours of incubation (Figure 4.13A and $\mathrm{B}, \mathrm{p}=0.024$ ). No significant difference was found between incubation at $4{ }^{\circ} \mathrm{C}$ and $20{ }^{\circ} \mathrm{C}$, likely because of the high variability of the $4{ }^{\circ} \mathrm{C}$ group but there was a trend of higher cell counts in the $4{ }^{\circ} \mathrm{C}$ group at most time points (Figure 4.13A). 
A
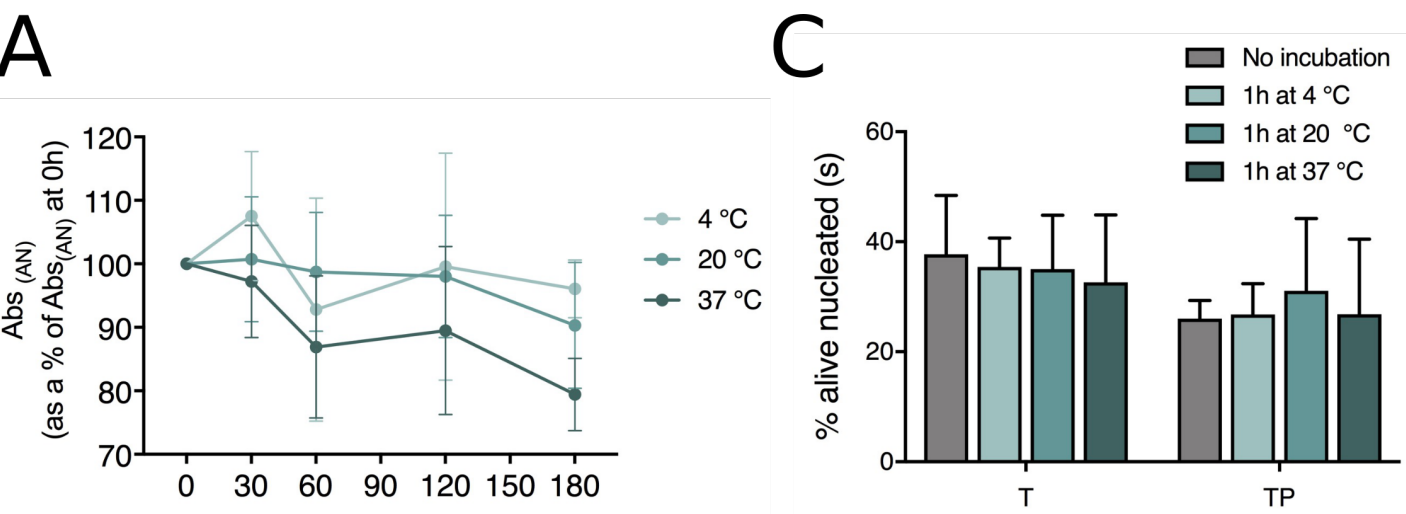

B

Time (min)

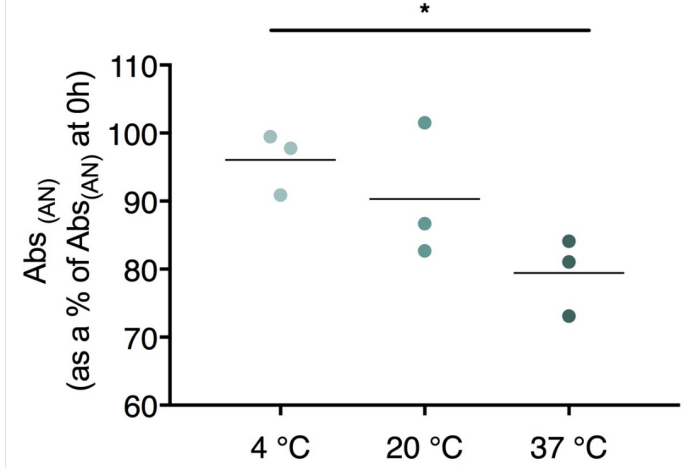

D

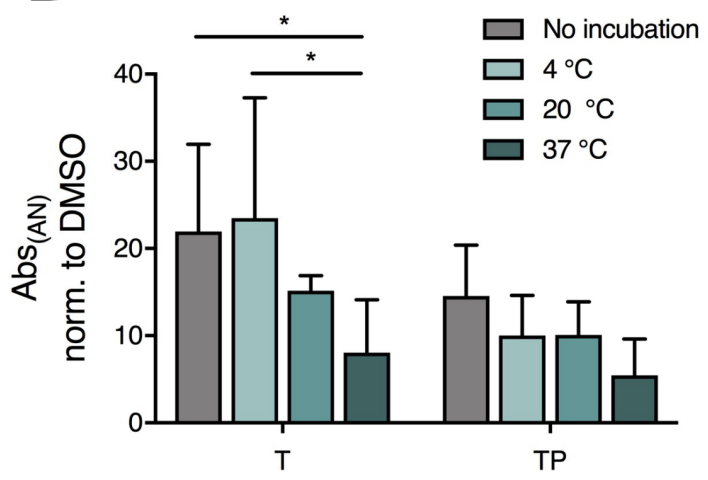

Fig. 4.13 Optimisation of incubation temperature before splenocyte cryopreservation. (A) Absolute count of alive nucleated cells $\left(7-\mathrm{AAD}^{-}\right.$Syto $\left.13^{+}\right)$normalised to 0 min time point after culture with $200 \mathrm{mM}$ trehalose at 3 different temperatures. (B) Absolute count of alive nucleated cells (7-AAD ${ }^{-}$ Syto $13^{+}$) after $180 \mathrm{~min}$ of culture in $200 \mathrm{mM}$ trehalose normalised to 0 min time point for 3 different temperatures. (C) Percentage of alive nucleated cells $\left(7-\mathrm{AAD}^{-}\right.$Syto13 $3^{+}$) out of all singlet events after cryopreservation of cells incubated for $1 \mathrm{~h}$ at 3 different temperatures. (D) Post-thaw absolute counts of alive nucleated cells (7-AAD Syto $\left.13^{+}\right)$after cryopreservation of cells incubated for $1 \mathrm{~h}$ at 3 different temperatures, normalised to DMSO control for each replicate. Full circles in (A) represent mean $\pm \mathrm{SD}(\mathrm{n}=3)$, (B) shows the results of each individual replicate with mean shown as a horizontal line, difference was tested by one-way repeated measures ANOVA followed by Tukey's HSD test. Bars in (C) and (D) represent mean $\pm \mathrm{SD}(\mathrm{n}=3)$, difference was tested by two-way ANOVA followed by followed by Tukey's HSD test, significance is marked by an asterisk: $* \mathrm{p} \leq 0.05$. 


\section{Cryopreservation with $\mathrm{T}$ and $\mathrm{TP}$ incubated at different temperatures}

Next, the splenocytes were incubated with trehalose (T) or trehalose + PP-50 (TP) for $1 \mathrm{~h}$ at $4{ }^{\circ} \mathrm{C}, 20^{\circ} \mathrm{C}$ or $37^{\circ} \mathrm{C}$, or frozen without any incubation. Cells were then cryopreserved and assessed for post-thaw absolute cell count and viability using Trucount tubes and Antibody panels B1 and B2 (Appendix B).

After $1 \mathrm{~h}$ incubation, no significant differences were found in the post-thaw percentage of alive nucleated cells for the three different temperatures (Figure 4.13C). Nevertheless, there was a significant temperature-related decrease in the absolute cell count in T. Again, the $4{ }^{\circ} \mathrm{C}$ group exhibited high variability. Similar trend, although without statistical significance, was observed in the TP group (Figure 4.13D). Overall, none of the conditions resulted in an increase in viability compared to no incubation control.

There are few previous reports of splenocyte culture (mouse or human) and previous studies have almost always included mitogens (such as phytohemagglutinin, concanavalin A, lipopolysacharide) or other factors (2-mercaptoethanol) in the culture medium to stimulate growth and activation of cells. Culture conditions of mononuclear cells from peripheral blood or bone marrow were also not systematically studied. Moreover, these results are not directly comparable with previous report as the culture medium was supplemented with trehalose during the incubation, which may exhibit an additional beneficial or toxic effect.

\section{Effect of culture with trehalose}

A series of experiments was next conducted to examine the survival of cells in culture with or without $200 \mathrm{mM}$ trehalose at room temperature. Absolute cell counts were measured at $2 \mathrm{~h}$, $24 \mathrm{~h}$ and $48 \mathrm{~h}$ using Trucount beads combined with Syto13 and 7-AAD staining of the cells.

While viability of cells in culture medium alone decreased significantly over time, addition of trehalose caused more rapid rate of cell death. However, this effect was negligible for a $2 \mathrm{~h}$ incubation time (Figure 4.14A). Change in light scatter characteristics of the cells could be observed, especially in the granulocyte population (Figure 4.14B) even at short incubation times.

Splenocytes cultured with culture medium only (without trehalose) show relatively high rate of cell death over a $48 \mathrm{~h}$ period. Majority of cells in spleen are differentiated cells unable to divide without specific stimuli. Moreover, there is significant stress involved during processing of the spleen into single cell suspension, accompanied by release of intracellular contents by necrotic cells which can trigger apoptosis or clumping of other surviving cells. For all these reasons, splenocytes generally do not tolerate long-term culture. Nevertheless, 

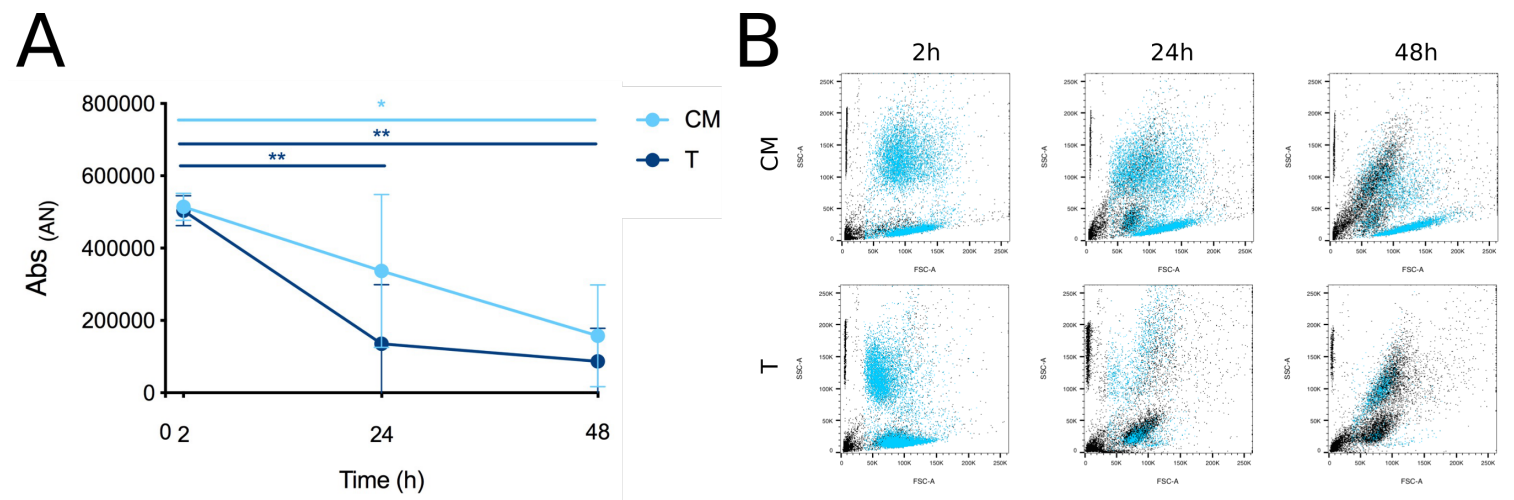

Fig. 4.14 Culture of splenocytes with culture medium or $200 \mathrm{mM}$ trehalose. (A) Absolute count of

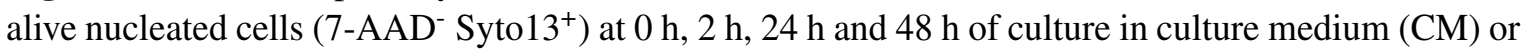
$200 \mathrm{mM}$ trehalose $(\mathrm{T})$. Full circles represent mean $\pm \mathrm{SD}, \mathrm{n}=3$, differences between conditions were tested by two-way repeated measures ANOVA followed by Tukey's HSD test, significance is marked by an asterisks: $* \mathrm{p} \leq 0.05 ; * * \mathrm{p} \leq 0.01$. (B) Flow cytometry plots (FSC vs. SSC) of splenocytes cultured in culture medium only $(0 \mathrm{mM})$ or $200 \mathrm{mM}$ trehalose for $2 \mathrm{~h}, 24 \mathrm{~h}$ or $48 \mathrm{~h}$. Alive nucleated cells $\left(7-\mathrm{AAD}^{-}\right.$Syto $\left.13^{+}\right)$are shown in blue and all other singlet events, including counting beads, debris, dead cells and erythrocytes are shown in black.

short incubation times should be possible without significant cell losses or manifestation of toxic effects of cryoprotectants.

With short incubation times at $20^{\circ} \mathrm{C}$, the cell death seems to be independent of trehalose (Figure 4.14A), although there appeared to be an effect specific for granulocytes (Figure 4.14B). It was also apparent from the culture experiments in Figure 4.13 that most of the temperature-related damage is caused during the incubation period and that this incubation does not result in increased cryoprotection for any of the temperatures tested. In two publications using permeabilising polymers, incubation time was reported to have beneficial effect on the cryosurvival of cells $[182,142]$. However, cells used in these studies (SAOS-2 cells and erythrocytes) survive well in culture compared to mononuclear cells.

There was no difference between $4{ }^{\circ} \mathrm{C}$ and $20{ }^{\circ} \mathrm{C}$ in the TP group and extensive cell death was also observed during culture at $37^{\circ} \mathrm{C}$. The toxic effect of DMSO is reportedly temperature-dependent [106], which may also be the case with trehalose. Use of low temperatures during incubation would therefore minimise toxicity but it must be highlighted that the ultimate goal was to optimise the incubation conditions for the function of the polymer. At low temperatures, diffusion rates of molecules are decreased (or halted) and the membrane becomes less fluid which will hypothetically decrease transport of trehalose through the membrane. Subsequent experiments were therefore conducted at room temperature and short incubation times to minimise trehalose toxicity and ensure rapid movement of molecules across the membrane. 
To conclude, incubation of splenocytes with trehalose at $37^{\circ} \mathrm{C}$ increased the rate of cell death and is therefore not recommended for pre-cryopreservation incubation. No significant difference was found between incubation at $4{ }^{\circ} \mathrm{C}$ and $20{ }^{\circ} \mathrm{C}$ which both worked better than $37^{\circ} \mathrm{C}$. However, none of the incubation temperatures provided an improvement which would justify for the inclusion of incubation time. Short incubations at $4{ }^{\circ} \mathrm{C}$ or $20{ }^{\circ} \mathrm{C}$ may be possible but there is no evidence that this incubation is beneficial for cryosurvival of splenocytes.

\subsubsection{Incubation $\mathrm{pH}$}

PP-50 and PP-75 polymers are $\mathrm{pH}$-sensitive and have been shown to only provide efficient intracellular loading of trehalose at $\mathrm{pH} 7.05$ or lower [125]. This could underlie the lack of efficacy of the polymer to improve cryosurvival was not observed when polymer was used in previous experiments. If splenocytes can survive a short period of incubation at $\mathrm{pH} 7.05$, and if this results in increased uptake of trehalose, this may lead to improved cryopreservation.

To determine the effect of $\mathrm{pH}$ on the survival of splenocytes during the incubation period, freshly isolated human splenocytes were resuspended in culture medium with or without $100 \mu \mathrm{g} / \mathrm{mL}$ PP-50 with $\mathrm{pH} 7.05$ or 7.4 and incubated at room temperature for $48 \mathrm{~h}$. At $2 \mathrm{~h}$, $24 \mathrm{~h}$ and $48 \mathrm{~h}$, the absolute count and viability were determined using Trucount beads and Syto13 + 7-AAD staining.

The result shows a decrease in the amount of alive cells when the $\mathrm{pH}$ of the medium is 7.05 compared to 7.4 but this is only observable at longer incubation times ( $24 \mathrm{~h}$ or $48 \mathrm{~h}$ ). Decrease can also be observed in the conditions where PP-50 has been added compared to the medium without PP-50. Overall, the best survival was shown for splenocytes incubated without PP-50 at $\mathrm{pH}$ 7.4. Short incubations $(2 \mathrm{~h})$ at either $\mathrm{pH}$ do not seem to affect the survival in culture (Figure 4.15A).

Next, human splenocytes were resuspended in $200 \mathrm{mM}$ trehalose with (TP) or without (T) $100 \mu \mathrm{g} / \mathrm{mL}$ PP-50 with $\mathrm{pH} 7.05$ or 7.4 and either frozen immediately using a freezing container or incubated for $1 \mathrm{~h}$ at room temperature and subsequently frozen. After thawing, absolute cell count and viability were determined using Trucount beads and B1 + B2 Antibody panels (Appendix B).

No differences were detected between the cells cryopreserved in the media of different $\mathrm{pH}$. Both the percentage of alive nucleated cells and their absolute number were similar for $\mathrm{pH} 7.05$ and 7.4, both with and without pre-freeze incubation with the polymer (Figures 4.15B, $\mathrm{C}$ and $\mathrm{D}$ and $\mathrm{E})$.

Lowering the $\mathrm{pH}$ to 7.05 has been shown to have a beneficial effect on trehalose loading in erythrocytes [125]. Erythrocytes have been shown to have stable properties over the $\mathrm{pH}$ 


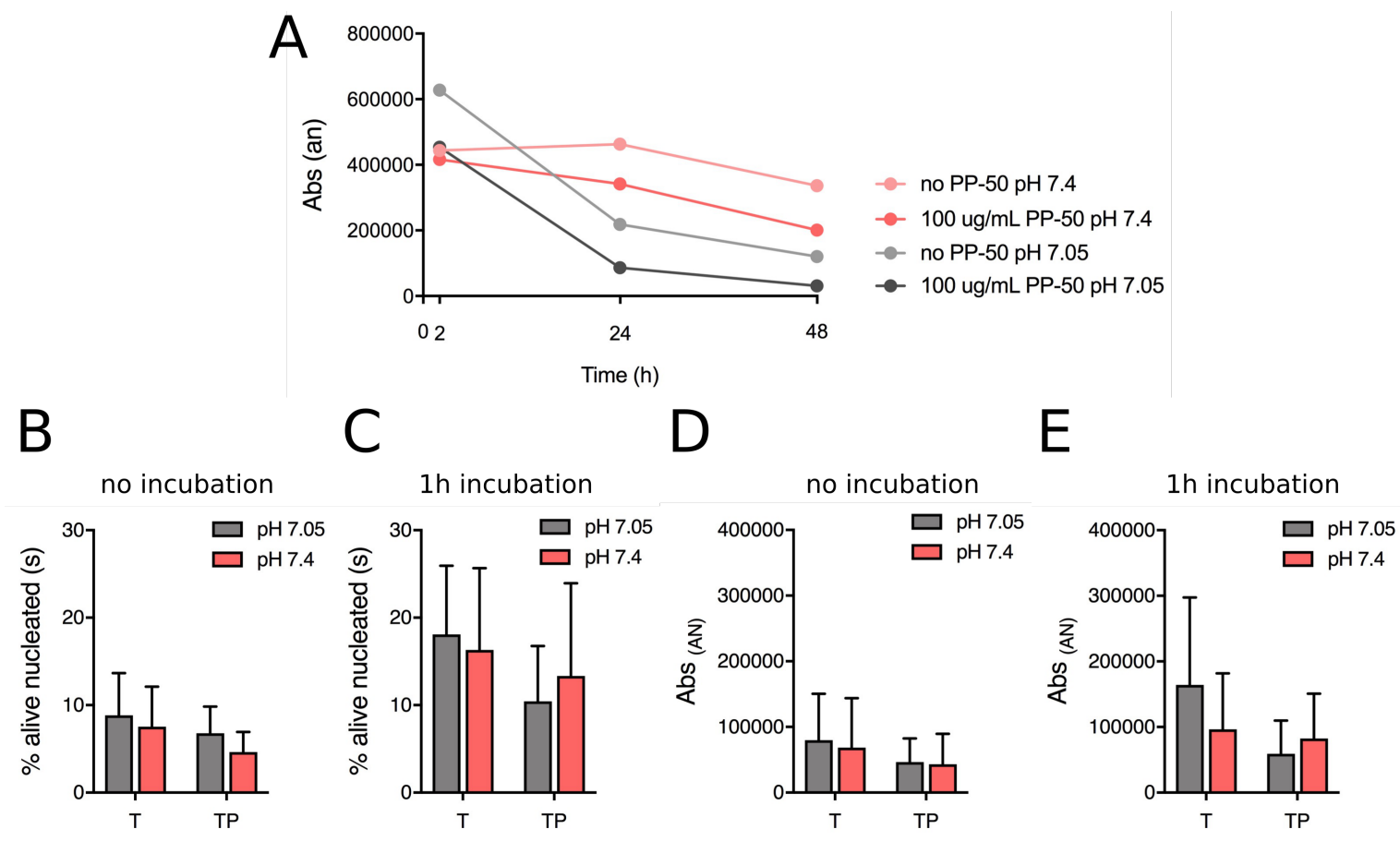

Fig. 4.15 Optimisation of incubation $\mathrm{pH}$ before splenocyte cryopreservation. (A) Absolute number of alive nucleated cells $\left(7-\mathrm{AAD}^{-}\right.$Syto $\left.13^{+}\right)$for splenocytes incubated for $2 \mathrm{~h}, 24 \mathrm{~h}$ or $48 \mathrm{~h}$ at $\mathrm{pH} 7.05$ or 7.4; with or without PP-50 $(\mathrm{n}=1)$. No statistical analysis could be performed due to a low number of replicates. (B) Post-thaw percentage of alive nucleated cells $\left(7-\mathrm{AAD}^{-}\right.$Syto $\left.13^{+}\right)$out of all singlet events for splenocytes without incubation. (C) Post-thaw percentage of alive nucleated cells (7-AAD ${ }^{-}$ Syto $13^{+}$) out of all singlet events for splenocytes with $1 \mathrm{~h}$ incubation. (D) Post-thaw absolute number of alive nucleated cells $\left(7-\mathrm{AAD}^{-}\right.$Syto13 $3^{+}$) for splenocytes without incubation. (E) Post-thaw absolute

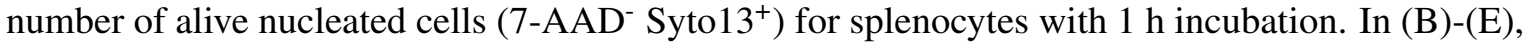
splenocytes were cryopreserved in $200 \mathrm{mM}$ trehalose with (TP) or without (T) PP-50; $\mathrm{pH}$ adjusted to 7.05 or 7.4 and vials either frozen immediately or after $1 \mathrm{~h}$ of incubation; $n=3$, in (B) - (D), statistical analysis performed using repeated measures two-way ANOVA followed by Sidak's correction, no significant differences were found. 
range of $6.3-7.9$ [183] and are therefore suitable for incubation at $\mathrm{pH}$ 7.05. Splenocytes which already have a high cell death rate in culture, appear to be more sensitive to this change of $\mathrm{pH}$ based on these experiments.

In summary, the data indicate that incubation of splenocytes at low $\mathrm{pH}$ leads to increased cell death pre-cryopreservation (time-dependent) with no beneficial effect on post-thaw survival when cryopreserved with trehalose and PP-50 polymer.

\subsection{Concentration of components}

\subsubsection{Concentration of trehalose}

The optimisation steps described earlier (cell density, addition of FCS, incubation time, temperature and $\mathrm{pH}$ or thawing technique) did not result in a significant improvement in the efficacy of the trehalose cryopreservation protocol. Another obvious variable which significantly contributes to the success of cryopreservation is concentration of the cryoprotectants. Trehalose was used at a concentration of $200 \mathrm{mM}$ in the previous experiments, based on previously published studies. The optimal concentration of trehalose has, however, not been determined for mononuclear cells, including splenocytes. This was explored in the next series of experiments.

\section{Cryopreservation with a range of trehalose concentrations}

Splenocytes were incubated with $0-800 \mathrm{mM}$ trehalose for 1 or $24 \mathrm{~h}$ at room temperature, before being frozen using a freezing container. Before cryopreservation and upon thawing, absolute number and percentage of alive nucleated cells were determined using Trucount beads and staining with Syto13 and 7-AAD.

The pre-cryopreservation absolute counts suggest that after a $1 \mathrm{~h}$ incubation, the number of viable cells in the sample is the same in all concentrations tested. However, longer incubations led to a decrease in the number of viable cells in samples in a concentrationdependent manner (Figure 4.16A).

After thawing, trehalose concentrations lower than $200 \mathrm{mM}$ did not seem to provide efficient cryoprotection. After a $1 \mathrm{~h}$ incubation, the percentage of alive nucleated cell increased with concentration in all three replicates, with the highest proportion found consistently in samples incubated in $800 \mathrm{mM}$ trehalose for $1 \mathrm{~h}$. For $24 \mathrm{~h}$ incubation, $200 \mathrm{mM}$ trehalose was the most effective but this longer incubation did not provide any added benefit compared to the $1 \mathrm{~h}$ incubation time (Figure 4.16B). The absolute counts after thawing were highly variable in the three donors but the general trend was that the most successful concentrations 

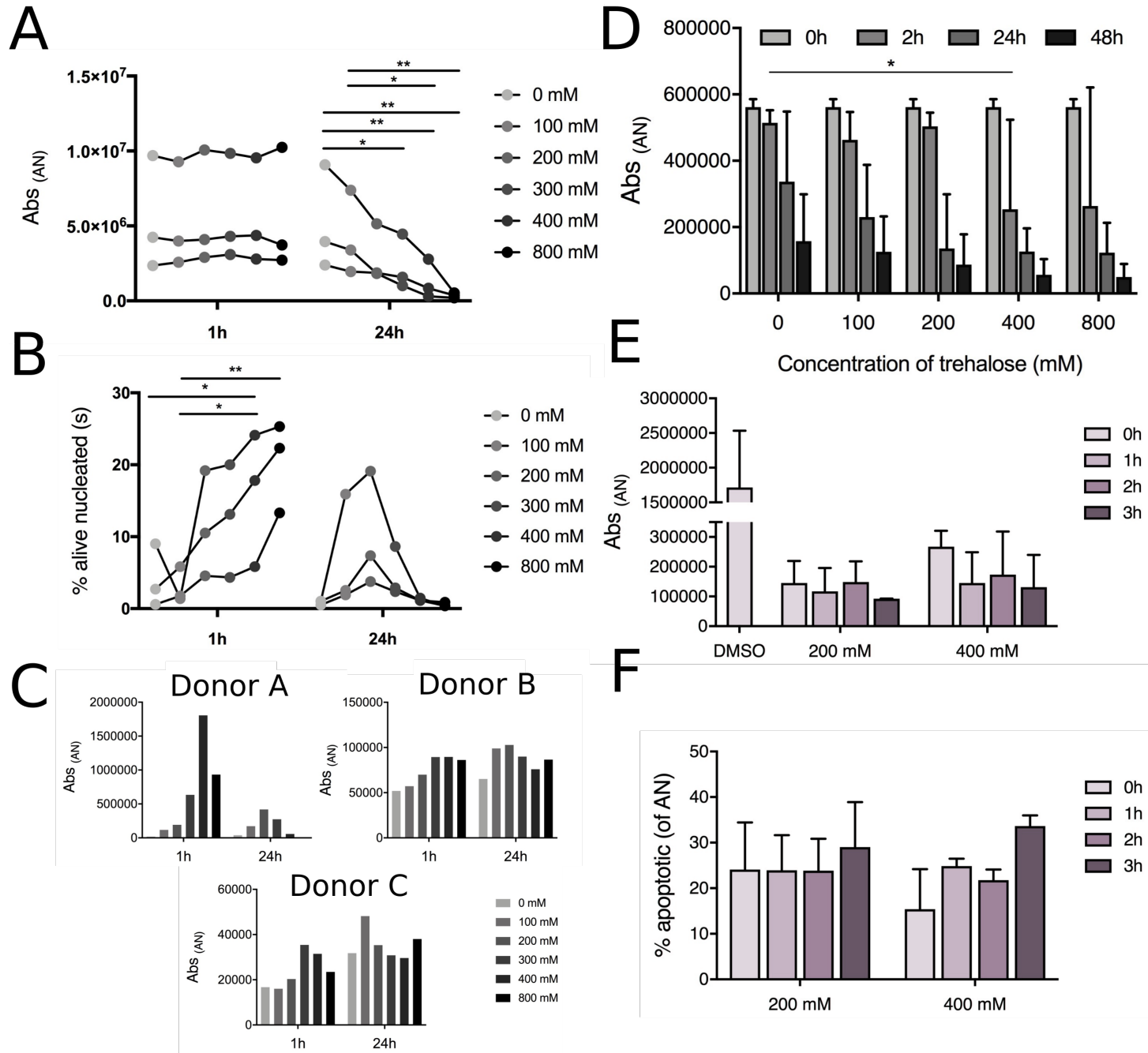

Fig. 4.16 Optimisation of trehalose concentration and incubation time. (A) Pre-freeze absolute number of alive nucleated cells $\left(7-\mathrm{AAD}^{-}\right.$Syto $\left.13^{+}\right)$incubated in $0-800 \mathrm{mM}$ trehalose for 1 or $24 \mathrm{~h}$. Full circles connected by a line represent data from a single replicate/donor. (B) Post-thaw percentage of alive nucleated cells $\left(7-\mathrm{AAD}^{-}\right.$Syto $\left.13^{+}\right)$out of all singlet events for samples incubated in $0-800 \mathrm{mM}$ trehalose for 1 or $24 \mathrm{~h}$. Full circles connected by a line represent data from a single replicate/donor. (C) Post-thaw absolute number of alive nucleated cells (7-AAD ${ }^{-}$Syto $\left.13^{+}\right)$incubated in $0-800 \mathrm{mM}$ trehalose for 1 or $24 \mathrm{~h}$. Individual replicates coming from different human donors shown in separate graphs. (D) Absolute numbers of alive nucleated cells (7-AAD Syto13 ${ }^{+}$) in toxicity assay performed on fresh splenocytes incubated with $0-800 \mathrm{mM}$ trehalose. (E) Post-thaw percentage of alive nucleated cells $\left(7-\mathrm{AAD}^{-}\right.$Syto $13^{+}$) out of all singlet events for samples incubated in $200 \mathrm{mM}$ or $400 \mathrm{mM}$ trehalose for $0,1,2$ or $3 \mathrm{~h}$. (F) Post-thaw percentage of apoptotic cells (7-AAD ${ }^{-}$Syto $13^{+}$ annexin $\mathrm{V}^{+}$) out of alive nucleated cells for samples incubated in $200 \mathrm{mM}$ or $400 \mathrm{mM}$ trehalose for 0 , 1,2 or $3 \mathrm{~h}$. Bars in (E)-(F) represent mean $\pm \mathrm{SD}$. In (A) and (B), $n=3$, two-way repeated measures ANOVA followed by Tukey's HSD test was performed. In (D), two-way repeated measures ANOVA followed by Dunnet's correction was used to compare the conditions to the control ( $0 \mathrm{mM}$ trehalose), significance is marked by an asterisks: $* \mathrm{p} \leq 0.05 ; * * \mathrm{p} \leq 0.01$. For $(\mathrm{E})$ and $(\mathrm{F}), \mathrm{n}=2$, no statistical analysis was performed. 
were between $300-400 \mathrm{mM}$ for $1 \mathrm{~h}$ incubation and $100-300 \mathrm{mM}$ for $24 \mathrm{~h}$ incubation (Figure 4.16C).

When examining the high percentage of alive nucleated cells in Figure 4.16B, it must be taken into consideration that this is a percentage of all singlet events. Theoretically, cells may undergo a very different fate in the case of $800 \mathrm{mM}$ to that of cells in $100 \mathrm{mM}$ trehalose. For example, cells which were already dead may have been so fragile after this considerable osmotic stress that they may have disintegrated and been washed away during the preparation for flow cytometry. This would in turn decrease the total number of events, giving a false perception of high viability.

It follows, therefore, that comparing the post-thaw absolute numbers is a more appropriate measure of efficacy of cryopreservation. Due to high variability between donors/preparations, it was not possible to pool the samples together and perform statistical analysis but it is still clear that in all three donors, $800 \mathrm{mM}$ trehalose was not the optimal concentration (Figure 4.16C).

Successful cryopreservation protocols aim to strike a balance between the efficacy of a cryoprotectant with the cryoprotectant toxicity. Both typically increase with the concentration of the cryoprotectant. This set of experiments confirmed that the efficacy of trehalose for cryopreservation increases with concentration at the $1 \mathrm{~h}$ incubation time, while this effect is reversed when longer incubation is used at higher concentrations. To confirm the concentration limit beyond which trehalose can be considered toxic, a set of experiments was designed to examine the effect of trehalose during culture over $48 \mathrm{~h}$.

\section{Trehalose toxicity}

Freshly isolated splenocytes from three human donors were seeded into a 96-well plate with $0-800 \mathrm{mM}$ trehalose and toxicity assay was performed as described in Methods Section 2.5.2. Measurements were taken at the beginning of the assay and at $2 \mathrm{~h}, 24 \mathrm{~h}$ and $48 \mathrm{~h}$.

No toxic effect was detected at $2 \mathrm{~h}$ time point for concentration of $200 \mathrm{mM}$ or lower but some level of toxicity was observed at $24 \mathrm{~h}$ and $48 \mathrm{~h}$ for all trehalose concentrations compared to the control. $400 \mathrm{mM}$ and $800 \mathrm{mM}$ concentrations exhibited the toxic effect at the $2 \mathrm{~h}$ time point, although this was highly variable in the three donors (Figure 4.16D).

While the reason for this toxicity of trehalose for splenocytes is unknown, it is possible that this toxicity is not due to specific trehalose properties but rather a non-specific effect of high concentration resulting in osmotic stress followed by cell shrinkage. However, the data suggests that it may be possible to use even these concentrations for short incubation times. 


\section{Incubation time with $400 \mathrm{mM}$ trehalose}

The incubation time experiments from Section 4.4.1 were therefore repeated, using only $200 \mathrm{mM}$ and $400 \mathrm{mM}$ trehalose concentrations and testing incubation times 0 to $3 \mathrm{~h}$ at room temperature.

There was no significant difference between $200 \mathrm{mM}$ and $400 \mathrm{mM}$ trehalose, both in terms of absolute number of alive nucleated cells and level of apoptosis in the post-thaw samples (Figures 4.16E and F). All absolute numbers were, however, still considerably lower than for cells cryopreserved with DMSO. Consistently with previous experiments, an increase in incubation time did not improve the outcome of cryopreservation (Figure 4.16F). The best overall result was achieved by $400 \mathrm{mM}$ trehalose without any incubation but this result was not significantly different from the other conditions.

While evidence from the literature typically suggests the use of $200 \mathrm{mM}$ concentration with regards to toxicity [54, 128], evidence from the above experiments suggests that it is possible to successfully use higher concentration if the incubation time is limited. This may be due to different susceptibility of other cell types (hepatocytes and TF-1 hematopoietic cell line in the mentioned publications).

In conclusion, cryopreservation with $400 \mathrm{mM}$ trehalose without incubation was identified as the most successful condition. If longer incubation are needed, the concentration must be reduced in order to avoid the concentration-dependent toxicity of trehalose. However, by changing trehalose concentrations, efficacy of cryopreservation cannot be significantly increased.

\subsubsection{Concentration of polymers}

Earlier experiments did not demonstrate any benefit of PP-50 permeabilising polymer over trehalose alone in improving post-thaw viability. The PP-50 concentration used for all experiments was $100 \mu \mathrm{g} / \mathrm{mL}$ based on previous reports [141]. I next explored whether different concentration of the polymer may be more effective in this human splenocyte model. Toxicity and efficacy of PP-50 for splenocytes was thus determined using range of concentrations of the polymer, together with a polymer variant, PP-75. This variant contains higher ratio of phenylalanine grafted onto the polymer backbone (PLP).

\section{Toxicity of PP-50 for splenocytes}

Freshly isolated splenocytes from three human donors were seeded into a 96-well plate with $0-1600 \mu \mathrm{g} / \mathrm{mL}$ of PP-50 or PP-75 and toxicity assay was performed as described in Methods 

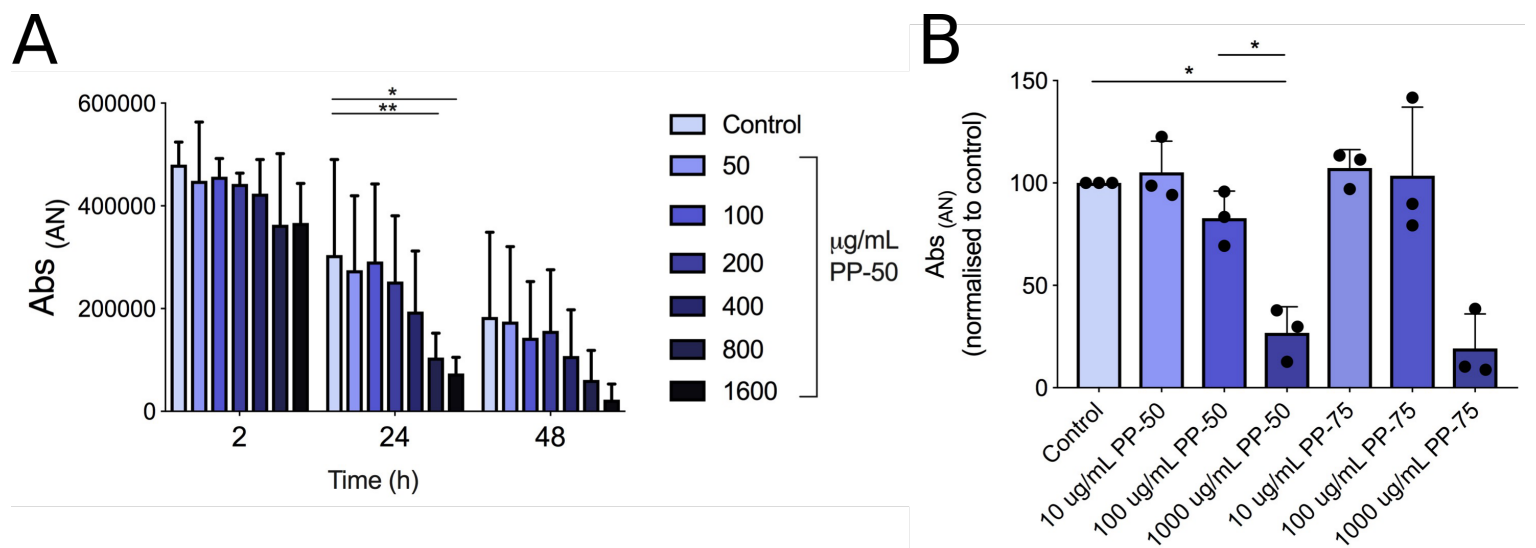

Fig. 4.17 Optimisation of PP-50 and PP-75 polymer concentration. (A) Absolute numbers of alive nucleated cells $\left(7-\mathrm{AAD}^{-}\right.$Syto $\left.13^{+}\right)$in toxicity assay performed on fresh splenocytes incubated with $0-1600 \mu \mathrm{g} / \mathrm{mL}$ PP-50. Bars represent mean $\pm \mathrm{SD}$, differences were compared to Control by repeated measures two-way ANOVA followed by Dunnett's correction. (B) Post-thaw absolute number of alive nucleated cells $\left(7-\mathrm{AAD}^{-}\right.$Syto $13^{+}$) incubated in $400 \mathrm{mM}$ trehalose with $0-1000 \mu \mathrm{g} / \mathrm{mL} \mathrm{PP-50}$ or PP-75 for $1 \mathrm{~h}$ at room temperature. Bars represent mean $\pm \mathrm{SD}$, full circles represent the individual replicates, data was normalised to Control (no polymer), differences were tested by repeated measures one-way ANOVA followed by Tukey's post-hoc test, significant differences are marked by asterisks: $* \mathrm{p} \leq 0.05 ; * * \mathrm{p} \leq 0.01$.

Section 2.5.2. Measurements were taken at the beginning of the assay and at $2 \mathrm{~h}, 24 \mathrm{~h}$ and $48 \mathrm{~h}$.

There was a concentration-dependent decrease in absolute number of surviving cells for all incubation times. For short-term $2 \mathrm{~h}$ incubation, PP-50 concentrations up to $400 \mu \mathrm{g} / \mathrm{mL}$ caused only a small decrease in viability. At longer incubation times ( $24 \mathrm{~h}$ and $48 \mathrm{~h}$ ), only concentrations of $200 \mu \mathrm{g} / \mathrm{mL}$ and lower did not lead to a significant drop in viability. Concentrations over $400 \mu \mathrm{g} / \mathrm{mL}$ of PP-50 showed high toxicity at all incubation times tested (Figure 4.17A). Results of the toxicity test with PP-75 were similar and are not shown.

In previous publications, PP-50 has been shown to be toxic for SAOS-2 cells in concentrations over $200 \mu \mathrm{g} / \mathrm{mL}$ after 2 and $24 \mathrm{~h}$ [142]. This is in agreement with the above experiments performed on splenocytes. This implies that use of concentrations over $200 \mu \mathrm{g} / \mathrm{mL}$ will result in decreased viability of the cells even before cryopreservation, if incubation period is included in the protocol. Previous publications on different cell types disagree on the ideal polymer concentration to be used for efficient uptake of trehalose and cryopreservation. Therefore, cryopreservation efficacy after addition of wide range of polymer concentrations to human splenocytes was next examined. 


\section{Concentration of PP-50 and PP-75 for cryopreservation}

Initial concentration of PP-50 was chosen based on previous publication as $100 \mu \mathrm{g} / \mathrm{mL}$ [182]. Short incubation was used in order to limit the cell death occurring during the culture of splenocytes. Human splenocytes from three donors were incubated for $1 \mathrm{~h}$ at room temperature with $0,10,100$ or $1000 \mu \mathrm{g} / \mathrm{mL}$ of PP-50 and cryopreserved using the freezing container. After thawing, absolute numbers of alive nucleated cells were determined using Trucount beads combined with Syto13 and 7-AAD staining.

For both PP-50 and PP-75, the absolute numbers of cells after thawing decreased with increasing concentration of the polymers. Post-thaw absolute numbers of splenocytes incubated at the highest concentration $(1000 \mu \mathrm{g} / \mathrm{mL})$ of PP-50 and PP-75 were very small $(26.76 \pm 12.79,(\mathrm{p}=0.0396)$ and $19.25 \pm 16.79 \%(\mathrm{~ns})$ of the control, respectively). The lowest concentration $(10 \mu \mathrm{g} / \mathrm{mL})$ of the polymers was comparable to the control without any polymer, although there was some variation in the individual replicates (Figure 4.17B).

The concentrations were chosen to cover a wide range and included toxic concentration of $1000 \mu \mathrm{g} / \mathrm{mL}$. Sharp et al. [142] have previously shown that PP-50 increased recovery of SAOS-2 cells after cryopreservation only for concentration of $25 \mu \mathrm{g} / \mathrm{mL}$ but not higher. Similar but very small effect was observed in some of the splenocyte donors. Overall, this effect was not consistent and not strong enough to justify for the use of the polymers in cryopreservation of splenocytes.

To conclude, PP-50 concentrations of $(200 \mu \mathrm{g} / \mathrm{mL})$ and lower did not decrease viability of splenocytes during the toxicity assay. However, improvement of trehalose cryopreservation has not been observed for non-toxic concentrations of 10 and $100 \mu \mathrm{g} / \mathrm{mL}$, regardless of the variant of the polymer (PP-50 or PP-75).

\subsection{Function and safety of permeabilising polymers}

\subsubsection{Trehalose uptake measurements by Raman spectroscopy}

To study the function of permeabilising polymers, their efficacy in delivering trehalose to the intracellular space must be tested. Methods for measurement of intracellular concentration of trehalose exist but they require lysis and often cannot distinguish between different saccharides. This project needed a method which would enable measurement of intracellular trehalose concentration not only for single cells but also multicellular aggregates, such as pancreatic islets. Raman spectroscopy was identified as a potential method and pilot experiments were performed on cancer cell lines since splenocytes would present additional challenges related to their availability and need for immobilisation on a substrate. 

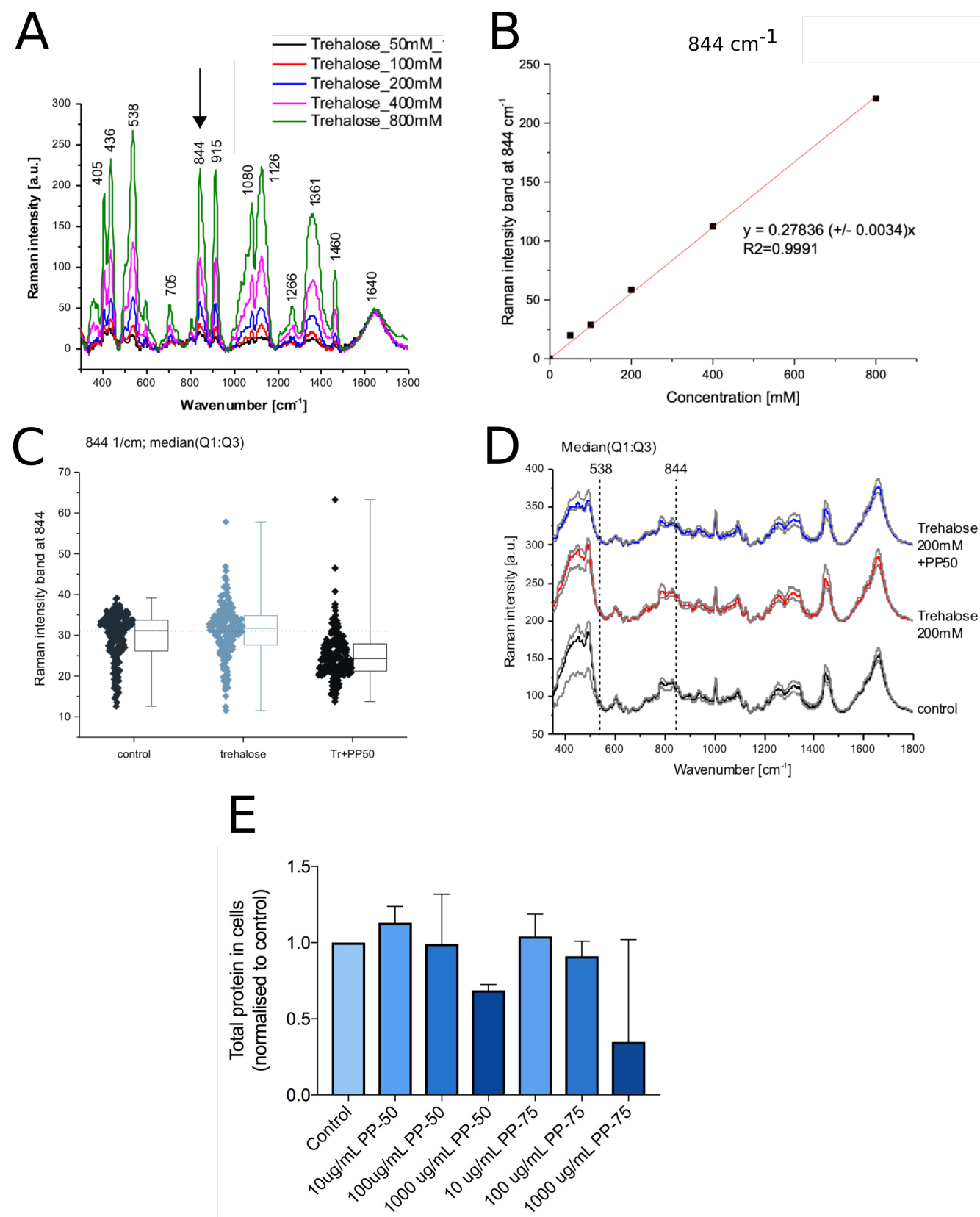

Fig. 4.18 Raman spectroscopy for trehalose uptake measurement and total protein assay. (A) Validation of Raman spectroscopy for measurement of trehalose: Raman spectra of $50-800 \mathrm{mM}$ trehalose solutions in $\mathrm{H}_{2} \mathrm{O}$; arrow shows the trehalose-specific peak of the Raman spectrum. (B) The linear relationship between trehalose concentration and the Raman intensity at the $844 \mathrm{~cm}^{-1}$ peak. (C) Raman intensities at the trehalose-specific peak $844 \mathrm{~cm}^{-1}$ for SAOS-2 cells incubated with culture medium (control), $200 \mathrm{mM}$ trehalose or $200 \mathrm{mM}$ trehalose $+100 \mu \mathrm{g} / \mathrm{mL}$ PP-50 for $6 \mathrm{~h}$. Boxplots show median and $1^{\text {st }}$ and $3^{\text {rd }}$ quartiles, 200 points across 10 cells for each condition were measured. (D) Raman spectra of SAOS-2 cells incubated with culture medium (control), $200 \mathrm{mM}$ trehalose or $200 \mathrm{mM}$ trehalose $+100 \mu \mathrm{g} / \mathrm{mL}$ PP-50 for $6 \mathrm{~h}$. Trehalose-specific peaks highlighted by vertical dotted lines. (E) Total intracellular protein concentration in splenocytes incubated with $0,10,100$ or $1000 \mu \mathrm{g} / \mathrm{mL}$ PP-50 for $4 \mathrm{~h}$ at room temperature. Bars represent mean $\pm \mathrm{SD}$, all values normalised to control, $\mathrm{n}=2$, no statistical tests were performed. 
All Raman spectroscopy experiments were performed and analysed by Jakub Surmacki from the Cavendish Laboratory, University of Cambridge. First, the Raman measurements of trehalose were validated using $50-800 \mathrm{mM}$ trehalose solutions and specific peaks were identified (Figure 4.18A). Identification of $844 \mathrm{~cm}^{-1}$ peak in cells was then validated using MDA-MB-231 cell line (data not shown).

SAOS-2 cells were prepared and measurements performed as described in detail in Methods Section 2.7.4. This experiment consisted of three groups of cells, incubated either in culture medium, $200 \mathrm{mM}$ trehalose or $200 \mathrm{mM}$ trehalose $+100 \mu \mathrm{g} / \mathrm{mL}$ PP-50 for $6 \mathrm{~h}$ in duplicates.

Raman intensities from the intracellular space of cells incubated with $200 \mathrm{mM}$ trehalose show that there is only a small number of measurements where the trehalose concentration was detected above the level of the control cells. While some very high Raman intensities were measured in the $200 \mathrm{mM}$ trehalose + PP-50 group, it is clear from the collected data that the background signal in this group has decreased when compared to the control and the $200 \mathrm{mM}$ trehalose group (Figure 4.18B).

It was also apparent from the average spectra of the three conditions where the peaks in $200 \mathrm{mM}$ trehalose + PP-50 appear blunted and lower than in the the other two conditions, that this is a trend across the whole spectrum, not only at the $844 \mathrm{~cm}^{-1}$ peak (Figure $4.18 \mathrm{C}$ ).

Raman spectroscopy is a powerful technique which is being developed for the purposes of many fields to measure concentrations of substances, increasingly also in living cells. Nevertheless, applying this technology for measurement of intracellular trehalose is very novel, being used only once in a study of spin-dried cells [184]. The challenge of this technique is to exclude the signal from the extracellular space since the ability of the instrument to exclude signal from the non-imaged layers (above and below the intracellular space) is limited. Extensive washing procedure was therefore employed in these experiments to reduce any strong trehalose signal from the extracellular space. Despite this challenge, these experiments demonstrate that it is possible to use this technique for a measurement of intracellular trehalose and it could be a useful non-destructive method for intracellular trehalose measurements in cryobiology and other fields.

Comparison of the $200 \mathrm{mM}$ trehalose and $200 \mathrm{mM}$ trehalose + PP-50 group was not possible due to the decrease in background signal in the latter group. Because this decrease was observed across the whole Raman spectrum, it could suggest a drop in concentration of different cellular components (proteins, lipids, small molecules), caused perhaps by a significant damage to the cellular membrane. While penetrating biopolymers such as PP-50 were developed to make plasma membrane more liquid for delivery of substances into the cells, it is not clear whether this also allows intracellular molecules to leak out of the cells. 
This leakage has not been previously examined in literature, but nonetheless could have implication for safety of these molecules in clinical use. On the other hand, this observation could be an artefact of the cell preparation if, for example, PP-50 made the cells more susceptible to membrane damage during the fixation process.

\subsubsection{Safety of permeabilising biopolymers}

To further explore the possibility of molecules leaking out of cells, total intracellular protein was measured after incubation of splenocytes with range of concentrations (10, 100 and $1000 \mu \mathrm{g} / \mathrm{mL}$ ) of PP-50 and PP-75. Cells were incubated with the polymer at room temperature for $4 \mathrm{~h}$ before washing and performing BCA total protein assay.

A trend in concentration-dependent decrease in total intracellular protein was observed for both PP-50 and PP-75. However, for 10 and $100 \mu \mathrm{g} / \mathrm{mL}$ concentrations, this decrease was insignificant compared to the control incubated without any polymers (Figure 4.18D).

While these results are preliminary, they would suggest that some level of molecule leaking from cells may be present during the use of permeabilising polymers PP-50 and PP75. This effect is likely to be more pronounced at higher concentrations of the polymer and presumably also at longer incubation times. Moreover, the leaking will likely be dependent on the size of molecules. It was shown for a similar polymer (PLP-NDA) in erythrocytes to selectively allow entry of dextran molecules up to $70 \mathrm{kDa}$ but not larger [185].

There are several potential safety risks associated with leaking of molecules outside of cells. First of all, these cells could potentially lose components critical to their function. Secondly, if these cells were used in vivo, there would be a risk of releasing factors (short nucleic acid strands, metabolites and small molecules) which can act as damage-associated molecular patterns and could result in inflammation or apoptosis of surrounding cells. Safety of these permeabilising polymers should therefore be thoroughly tested before they are used as part of therapies.

\subsection{Discussion}

\subsubsection{Trehalose as an alternative to DMSO}

The cryopreservation efficacy of DMSO is currently sufficient to provide viable CD34 ${ }^{+}$cell numbers needed for clinical transplantation. As it was demonstrated in Section 4.3, the current DMSO protocol could only be improved marginally by optimisation of thawing protocol, cell density or addition of FCS. Nevertheless, the need to improve this protocol 
remains because of the demonstrated cellular and systemic toxicity of DMSO. One of the approaches to do this is the use of an alternative non-toxic cryoprotectant.

The experiments in this Chapter explored this approach by completely substituting DMSO for disaccharide trehalose. Trehalose has been previously tested on mononuclear cells or their subsets in combination with DMSO [131, 132, 186] or on its own [130]. While the observations from these publications confirm that trehalose on its own does not match to DMSO in its cryopreservation efficacy, none of the publications attempted a comprehensive and systematic optimisation of trehalose cryopreservation like the one performed in this project.

Numerous parameters were optimised in the current experiments, including thawing protocol, incubation time, temperature, $\mathrm{pH}$ and concentration. The parameter which had the biggest influence on the success of trehalose cryopreservation are trehalose concentration (average increase by $101.2 \%$ from $200 \mathrm{mM}$ to $400 \mathrm{mM}$ ). Nevertheless, the post-thaw absolute numbers of cells cryopreserved in trehalose $(400 \mathrm{mM}$ trehalose, $0 \mathrm{~h}$ incubation, optimised thawing, $10^{7}$ cells $/ \mathrm{mL}, 10 \%$ FCS) were on average still only $18.5 \%$ of the number of cells which survived freezing by DMSO. While the possibility of trehalose efficacy as a sole cryoprotectant can still not be ruled out, the results from this project do not support its use for cryopreservation of mononuclear cells, partially because of the difficulty with incubation of these cells.

Other approaches of minimising DMSO toxicity will therefore need to be explored. These could include combining DMSO with another cryoprotectant while decreasing DMSO concentration or development of efficient thawing and washing protocols which will minimise the cell losses and toxicity of DMSO.

\subsubsection{PP-50 as a method of intracellular trehalose delivery}

One of the approaches which could still improve trehalose performance in cryopreservation of cells is intracellular trehalose delivery. While a variety of approaches for this exists, the one explored in this project was the use of membrane permeabilising biopolymers PP-50 and PP-75.

These polymers have previously been shown to improve cryopreservation of red blood cells [141]. However, the data collected here does not confirm this efficacy in mononuclear cells. There are some suggestions that in nucleated cells, which are capable of endocytosis, the localisation of these polymers is not on the membrane [146]. The attached polymers appear to be endocytosed and based in small vesicles close to the cell nucleus which could potentially prohibit the intended function of being the gateway of trehalose on the cell surface. If the same rate of endocytosis was maintained whether or not the polymer is present, it is not 
clear how the polymer would encourage the increased uptake of trehalose. It is, however, also possible that the polymer encourages endocytosis and extracellular trehalose is transported to the cell interior in the central part of these vesicles. The details of this have not been previously investigated.

Another class of PLP backbone-based polymers has been developed and its journey along the endocytosis pathway further investigated. Along the endosome journey towards a more acidified lysosome, polymers change their conformation and help to release the intravesicular contents into the cytoplasm [185]. This would presumably release the trehalose to the cytoplasm. Nevertheless, Mercado et al. [146] have observed localisation of trehalose only in these intracellular vesicles.

While most nucleated cells possess the ability to endocytose, some may do this more often than others and if endocytosis is indeed involved in the function of these polymers, it may be difficult to work with mixed cell populations such as mononuclear cells where the extent to which each cell type can endocytose differ. Moreover, endocytosis is temperature-dependent and is almost halted at temperatures below $10{ }^{\circ} \mathrm{C}$ [187]. Lynch et al. [141] have shown that the maximum effect of polymers was achieved at incubation temperature of $37^{\circ} \mathrm{C}$ and long incubation times of nine hours. However, experiments with splenocytes described in Section 4.4 have shown that incubation at $37^{\circ} \mathrm{C}$ and for prolonged periods of time results in reduced viability.

\subsubsection{Safety of the new protocol}

While the function of permeabilising polymers should be further studied and optimised for different nucleated cell types, assessing safety of these compounds is of utmost importance if they are to be used as part of clinical therapies.

First, correct concentrations of the components must be established. While the toxic concentrations of trehalose and the polymers were tested in this project by measuring viability of cells, there may be more subtle effects which could only be detected by more sensitive assays (RNA sequencing or functional assays such as CFUs for HSCs, immune cell activation assays) or in long term studies. Long term survival and function of any cryopreserved cell must be always evaluated before translation into a therapy.

Second, the possibility of washing off the polymer must be explored. Although it was suggested in the early publications about the polymers that these can be easily removed from the cells by changing the $\mathrm{pH}$ [141], it is not clear how this relates to the polymer molecules which have been endocytosed. It must also be taken into account that some cell types may not well tolerate $\mathrm{pH}$ changes. 
Finally, other potential risks must be ruled out. One of these is leakage of molecules from the cells to the extracellular space. Chen et al. [185] have shown that after incubation of erythrocytes with PLP-NDA, they were able to create so called 'erythrocyte ghosts'. These are erythrocyte-like structures consisting of erythrocyte membrane which released intracellular contents and are now filled with extracellular medium. PLP-NDA shares the same PLP backbone with PP-50 and PP-75 but is grafted with hydrophobic decylamine instead of simple hydrophobic amino acids. While their efficacy in membrane destabilisation can differ, the functional principal remains the same and both could therefore pose the same safety risks. Leakage of molecules important for the cell survival and function and release of inflammatory molecules should be thoroughly examined before the work with these polymers proceeds.

\subsection{Conclusion}

Current protocols for cryopreservation of mononuclear cells utilise DMSO as a cryoprotectant. DMSO, however, is toxic to cells and patients and therefore trehalose was selected as a possible alternative cryoprotectant. By systematic screening of protocol variations, including incubation parameters, composition of cryopreservation medium and concentration of components, this project has shown that trehalose as a sole cryoprotectant does not provide protection for cryopreservation of human mononuclear cells from spleen comparable to the current protocol using DMSO.

Addition of permeabilising polymers PP-50 and PP-75 was tested to allow for intracellular trehalose delivery, which should improve cryoprotection with trehalose. Experiments featuring a variety of concentrations, incubation times and conditions showed that these polymers do not provide this protective effect in splenocytes. Moreover, a concentration- and time-dependent toxic effect of the polymers were identified, as well as a potential side-effect of the permeabilising polymers resulting in intracellular molecule leakage. The use of polymers in cryopreservation of primary mononuclear cells can therefore not be justified based on these experiments. 


\section{Chapter 5}

\section{Developing a novel islet cryopreservation protocol}

\subsection{Introduction and aims}

Efforts to cryopreserve pancreatic islets started in the 1980s and peaked in 1990s, led by the groups of Dr. Raymond V. Rajotte, Dr. Jonathan R. T. Lakey and others. These resulted in development of a protocol utilising DMSO which is now considered the gold standard in the field [73]. This protocol was however never properly introduced into clinical practice due to suboptimal post-thaw survival of islets and the use of FCS [26]. In 1997, Beattie et al. reported that addition of trehalose to the DMSO cryopreservation protocol resulted in successful cryopreservation [83]. Since 1990s, the research in islet cryopreservation has not experienced any notable progress and these promising results using trehalose addition were not validated in further studies. The full potential of cryopreservation of islets with trehalose has, therefore, not been explored.

The objective of this chapter was to validate the current standard (DMSO) method of pancreatic islet cryopreservation [73] by systematic screening of variables and to explore the potential of cryopreservation with trehalose alone or its addition to the standard DMSO-based protocol. Where possible, the results were replicated using human islets but this could not be achieved for all the experiments due to limited availability of human pancreatic islets.

The first aim was to determine the safety of cryoprotectants used in this chapter: DMSO and trehalose. Results of these experiments are presented in Section 5.2 and used to inform optimal incubation times with the cryoprotectants in subsequent sections of this Chapter.

The second aim was to optimise the parameters for a DMSO-only protocol. This was undertaken because these optimisations were either not clearly defined in literature or their 
modification could potentially simplify and/or improve the final protocol. These included the methods of DMSO addition, DMSO removal and comparison of controlled vs. uncontrolled freezing of pancreatic islets. Results of these experiments are presented in Section $\mathbf{5 . 3}$ and the findings were used to inform all following experiments of this and following chapters.

The potential of trehalose to fully substitute for DMSO was then investigated in Section 5.4. Next, trehalose was explored as an additive to the optimised DMSO protocol in Section 5.5. Among the variables, incubation time and concentrations of the cryopreservation solution components were explored to ensure high survival of cells and minimal cellular stress and toxicity. In Section 5.6, the capacity of antioxidants salidroside and MitoQ to further improve the post-thaw survival of pancreatic islets was examined.

Finally, in vitro and in vivo viability and function of the islets cryopreserved using the newly developed successful protocols was tested by a range of assays to demonstrate the potential of this method for clinical use in Section 5.7.

\subsection{Toxicity of cryoprotectants for pancreatic islets}

Cryopreservation of cell clusters requires prolonged incubation with cryoprotectants before freezing to allow their diffusion throughout the cluster. The cryoprotectants are also necessarily present after thawing, before they diffuse out from the cells and are substituted by non-toxic media. It is well-known that DMSO is toxic to other cell types at the concentrations used for cryopreservation and cryopreservation protocols aim to minimise the cells' exposure time to DMSO. However, the toxicity of $2 \mathrm{M}$ DMSO (used in published pancreatic islet cryopreservation protocols) has not been comprehensively studied. Quantifying the toxicity of DMSO to pancreatic islets is essential for the design of cryopreservation protocols. Moreover, safety of trehalose must also be demonstrated to confirm that any damage to cryopreserved islets is not caused by trehalose during the pre-incubation. The survival of mouse and human pancreatic islets in a presence of a range of concentrations of trehalose $(0-800 \mathrm{mM})$ and 2 M DMSO was therefore tested, using the protocols described in Section 2.6.5.

\subsubsection{Toxicity of DMSO}

No decrease in viability was observed in the control islets incubated with standard culture medium for both mouse and human islets. While $2 \mathrm{~h}$ incubation with DMSO in human islets caused a non-significant decrease in viability from $95.4 \pm 5.0 \%$ to $88.7 \pm 5.5 \%$ (Figure 5.1B), the same incubation caused statistically significant drop in viability of mouse pancreatic islets to $78.5 \pm 12.9 \%$ (Figure 5.1A). Similarly, at $24 \mathrm{~h}$, the viability of mouse 

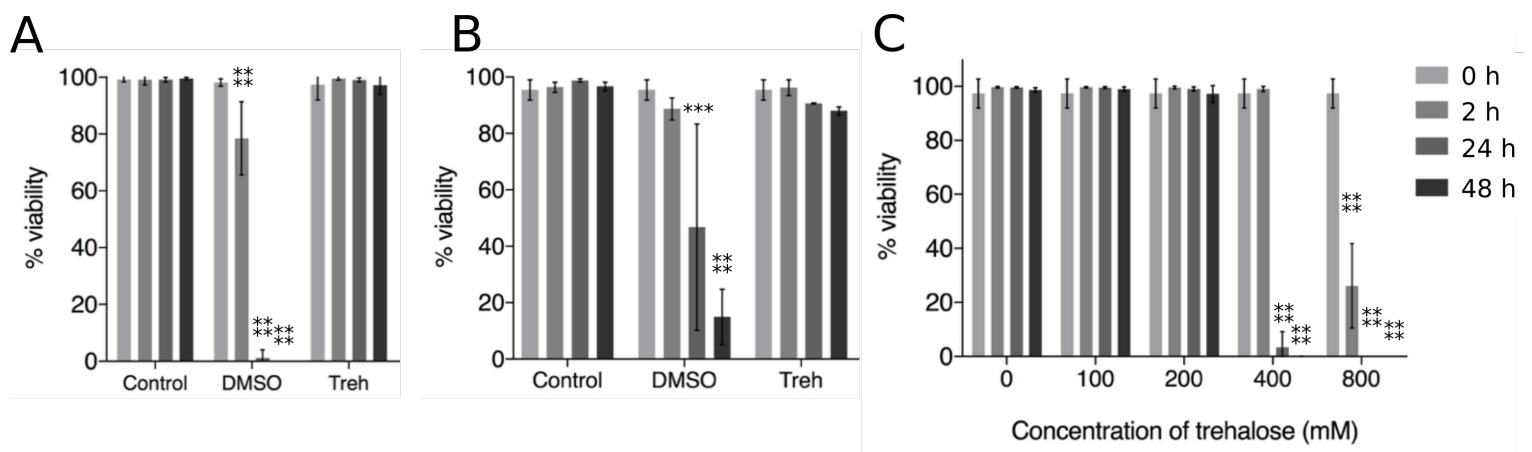

Fig. 5.1 Toxicity of DMSO and trehalose for human and mouse pancreatic islets after 2, 24 and $48 \mathrm{~h}$ incubation. (A) Viability of mouse pancreatic islets after incubation with $2 \mathrm{M}$ DMSO and $200 \mathrm{mM}$ trehalose (mean \pm SD of one experiment shown, viability of each experimental condition calculated as an average viability of $5-12$ islets). (B) Viability of human pancreatic islets after incubation with $2 \mathrm{M}$ DMSO and $200 \mathrm{mM}$ trehalose (mean \pm SD of two experiments shown, viability of each experimental condition calculated as an average of $3-13$ islets). (C) Viability of mouse pancreatic islets after incubation with different concentrations of trehalose $(0-800 \mathrm{mM}$; mean $\pm \mathrm{SD}$ of one experiment shown, viability of each experimental condition calculated as an average of $1-12$ islets). Differences between each group and control group (Control or $0 \mathrm{mM}$ trehalose) were tested by two-way ANOVA followed by Dunnett's test. Significant differences are marked by asterisks: $* \mathrm{p} \leq 0.05, * * \mathrm{p} \leq 0.01$; $* * * \mathrm{p} \leq 0.001 ; * * * * \mathrm{p} \leq 0.0001$. Treh $=$ trehalose.

islets was already below $2 \%$ but in human islets, the survival was $83.3 \%$ and $10.19 \%$ in the first and second repeat of the experiment, respectively. After $48 \mathrm{~h}, 24.8 \%$ and $5.2 \%$ viable cells could still be find in the human islets incubated with $2 \mathrm{M}$ DMSO in the two repeats of the experiment (Figure 5.1B).

As expected, DMSO exhibited acute toxic effects at $2 \mathrm{M}$ concentration: a decrease in viability could be observed after $2 \mathrm{~h}$ in both mouse and human islets. In human islets, there was significant variability between the two experiments performed, suggesting that different batches of islets could react differently to this treatment (possibly due to different level of damage caused to the islets during their isolation or transport or donor-specific features). Further repeats of these experiments are required to determine the extent of toxicity of DMSO for human islets. Pancreatic islet cryopreservation protocols require 45 min incubation with DMSO (although the protocol starts at lower concentration and is increased over time) and while this will likely result in some damage to the islets, sufficient islet mass will still be viable before cryopreservation. Human and mouse islets, although similar in size, differ in their cell composition, architecture or gene expression [188, 189]. As an example, human islets are known to contain higher proportion of $\alpha$-cells [188]. Since sensitivity to toxic effect of DMSO of different cell types differs, it would not be surprising if this is the case for the different cell types within pancreatic islets. This could be determined by studying the survival of the $\alpha$-and $\beta$-cell subsets but was not within the scope of this study. 


\subsubsection{Toxicity of trehalose}

For mouse islets, there was no change in viability after incubation with $200 \mathrm{mM}$ trehalose but toxic effects were noted at concentration of $400 \mathrm{mM}$. The islets maintained high viability after short incubation at $400 \mathrm{mM}$ but dropped to $3.4 \pm 5.8 \%$ viability after $24 \mathrm{~h}$ (Figure $5.1 \mathrm{C}$ ). In $800 \mathrm{mM}$ trehalose, a significant decrease in viability (to $26.2 \pm 15.6 \%$ ) could already be observed after $2 \mathrm{~h}$ of incubation and no viable islets were detected at $24 \mathrm{~h}$ and $48 \mathrm{~h}$. Due to the scarcity of human islets, a single concentration of trehalose was tested (200 mM). There was a slow, non-significant decrease in viability during incubation with trehalose, from $95.4 \pm 5.0 \%$ at $0 \mathrm{~h}$, to $88.0 \pm 2.1 \%$ at $48 \mathrm{~h}$ (Figure 5.1B).

The experiments suggest that toxic effects of trehalose manifest at concentrations over $200 \mathrm{mM}$ and at longer incubation times $(24 \mathrm{~h})$. Concentrations up to $200 \mathrm{mM}$ showed no toxic effect over the course of $48 \mathrm{~h}$. Toxicity of trehalose likely results from the impermeability of cell membrane to trehalose. High concentrations in the extracellular space cause strong concentration gradient, efflux of water from cells and result in shrinkage of cells in this hypertonic solution. The only available study on islet cryopreservation with trehalose (combined with DMSO) used $300 \mathrm{mM}$ trehalose and $2 \mathrm{M} \mathrm{DMSO}$, incubated for $45 \mathrm{~min}$ before freezing. Although the toxicity of trehalose was not specifically tested in this study, successful cryopreservation was achieved using this solution, suggesting that there was little or no toxicity to the islets before cryopreservation [83].

In summary, the experiments confirmed that DMSO is acutely toxic to mouse and human pancreatic islets, but short incubations $(<2 \mathrm{~h})$ may be possible to enable uptake of cryoprotectant by the islets. Pancreatic islets can be safely incubated with up to $200 \mathrm{mM}$ trehalose for $48 \mathrm{~h}$ and possibly with higher concentrations (300 or $400 \mathrm{mM}$ ) if the incubation time is shortened accordingly.

\subsection{Optimisation of DMSO protocol parameters}

\subsubsection{Addition of DMSO}

In cryopreservation of single cells, prolonged incubation with penetrating cryoprotectants is not needed before freezing. However, the size and architecture of pancreatic islets requires prolonged incubation to allow diffusion of solutes into the centre of the cell cluster. It is reasonable to assume that without penetration of the cryoprotectant into the islet core, the central cells would remain unprotected by the cryoprotectant. On the other hand, prolonged incubation may result in increased cellular toxicity (as described in Section 1.6.2). A protocol 


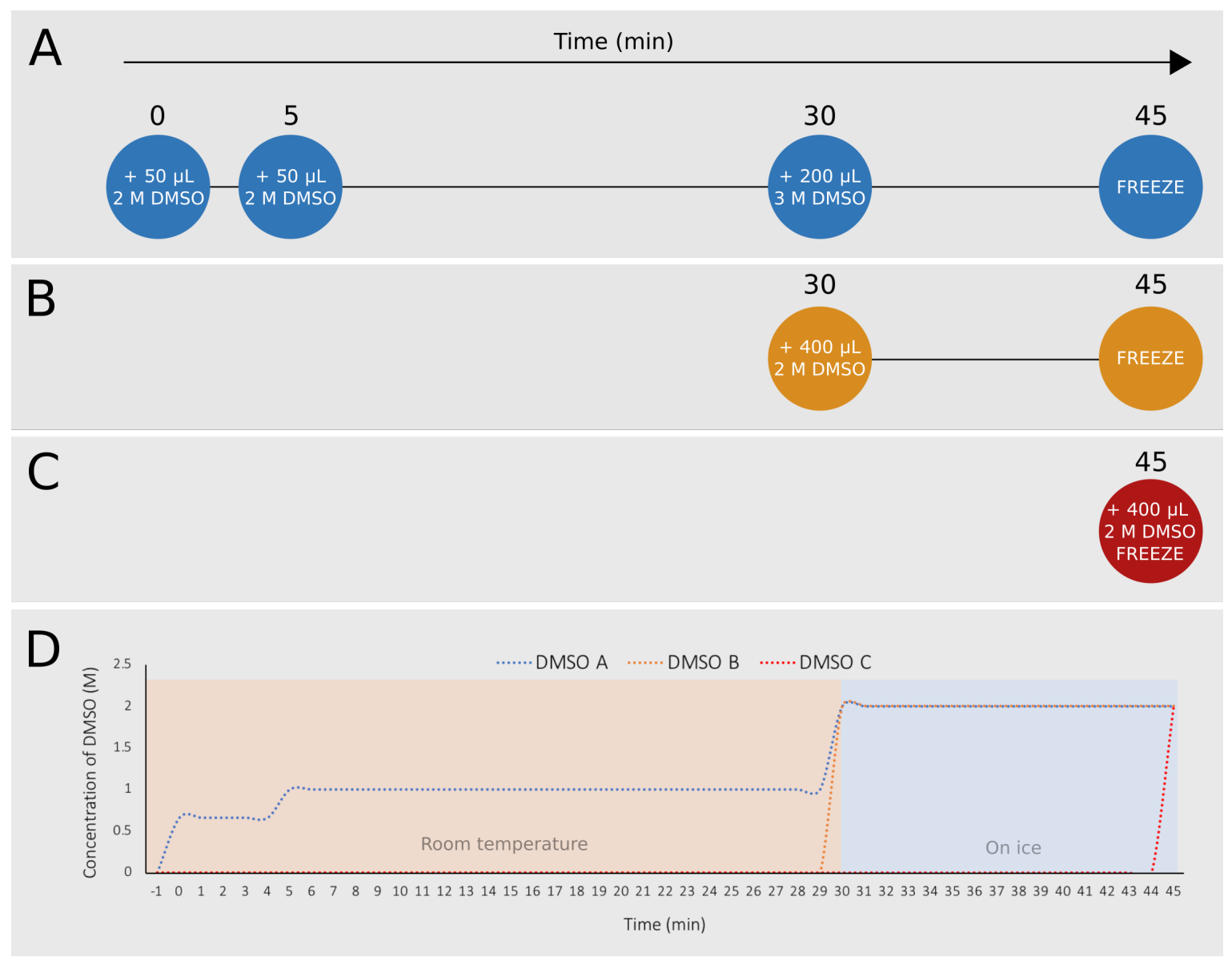

Fig. 5.2 Design of the DMSO addition protocol study. Time 0 represents the starting point of the DMSO addition to islets suspended in culture medium $(100 \mu \mathrm{L}$ for Protocol A but all medium removed for Protocols B and C). Each colourful circle represents an addition of DMSO (in $2 \mathrm{M}$ or $3 \mathrm{M}$ concentration) and numbers above the circles represent minute from time 0 at which the addition was performed. The three protocol require different time and the shorter protocols were therefore started at later time points to aim for the same freezing time. (A) Schematic of Protocol A: Three-step addition of DMSO over 30 min with 15 min equilibration before freezing. (B) Schematic of Protocol B: Singlestep addition of DMSO with 15 min equilibration before freezing. (C) Schematic of Protocol C: Single-step addition of DMSO without equilibration. (D) Model of changes of concentrations of DMSO over time using the three different DMSO addition protocols. For the first $30 \mathrm{~min}$, samples are kept at room temperature and they are moved onto ice for the final 15 min equilibration. 
including step-wise addition followed by equilibration protocol has been suggested by Rajotte [190] and is used widely in the field.

Three different DMSO addition protocols were compared, which differed in the number of DMSO addition steps and the length of equilibration (Figure 5.2). The final concentration of DMSO in all three protocols was $2 \mathrm{M}$ and the samples followed the same freezing and thawing procedure. Protocol A included step-wise addition of DMSO over $30 \mathrm{~min}$, followed by 15 min of equilibration on ice (the Rajotte's protocol [190]). In Protocol B, DMSO was added in single step and sample is equilibrated on ice for 15 min prior to freezing. In Protocol C, DMSO was again added in a single step but no equilibration period followed before freezing. It was hypothesised that that whole islets require a period of equilibration as a consequence of their 3D architecture rather than the individual islet cell properties. The three DMSO addition protocols were therefore tested using both whole pancreatic islets and dissociated islet cells.

\section{Whole islet cryopreservation}

In mouse islets, Protocol A resulted in post-thaw viability of $56.5 \pm 17.1 \%$, Protocol B in viability of $51.7 \pm 22.6 \%$ and Protocol C $54.9 \pm 12.3 \%$ (Figure 5.3A). After three days in culture, average viability dropped in all three protocols by approximately $28 \%$. No significant differences between protocols were detected.

In human islets, immediate post-thaw viability was $69.6 \pm 9.2 \%, 68.8 \pm 10.0 \%$ and $50.8 \pm 6.9 \%$ for protocols A, B and C, respectively (Figure 5.3B). After three days in culture, the viabilities in all three protocols were approximately the same in all three conditions. The data were not statistically analysed due to the low number of repeats because of limited availability of human islets.

There are a number of mathematical models to describe the diffusion of DMSO into tissues, but experimental evidence is limited. Benson et al. observed that $1.5 \mathrm{~mol} / \mathrm{kg}$ DMSO can equilibrate with an islet of $150 \mu \mathrm{m}$ diameter in about $600 \mathrm{~s}$ at room temperature [72]. Longer incubation times can nonetheless be necessary due to the use of different, gradually increasing DMSO concentrations in the protocol. Based on this model, Protocol B may be expected to be as effective as Protocol A, as both provide sufficient time for equilibration with DMSO. However, addition of $2 \mathrm{M}$ DMSO in a single step causes rapid osmotic change which could be detrimental to some cells.

In the absence of previous studies comparing different DMSO addition protocols, the optimal DMSO addition protocol for further studies was selected as Protocol A based on the data generated from these experiments. The results from mouse islet cryopreservation were inconclusive: All three protocols showed similar post-thaw survival both immediately and 

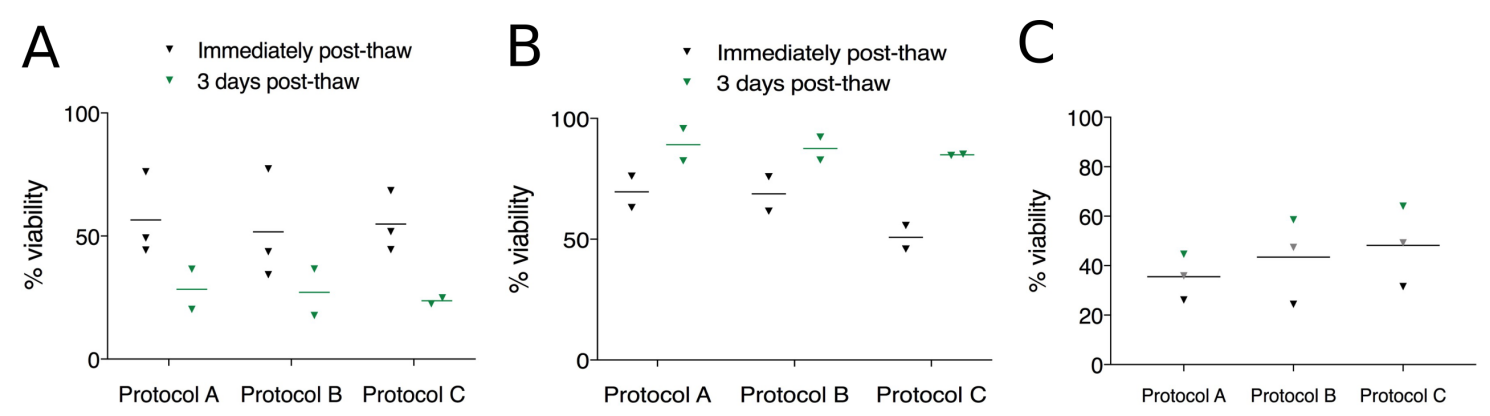

Fig. 5.3 Comparison of DMSO addition protocols for mouse and human pancreatic islets. Post-thaw viability of mouse (A) and human (B) pancreatic islets immediately after thawing and 3 days after thawing. Each triangle represents the average viability from one experimental sample; horizontal line represents the mean $(n=2-3$, viability of each experimental condition calculated as an average viability of 5-14 mouse islets or $8-18$ human islets). (C) Post-thaw viability of mouse dissociated pancreatic islet cells immediately post-thaw. Each triangle represents a single sample's viability, horizontal line represents the mean, different repeats of the experiment are shown in different colours $(n=3)$. Statistical comparison of groups in $(C)$ was performed using one-way repeated measures ANOVA followed by Tukey's HSD test. No statistical differences were found.

$24 \mathrm{~h}$ post-thaw. In human islets, Protocols A and B were equivalent. Protocol C (single-step addition without equilibration) was slightly inferior to the other two tested protocols where equilibration step was included.

\section{Dissociated islet cryopreservation}

In dissociated mouse islet cryopreservation, longer incubations with DMSO before freezing resulted in trend towards decreased viability of cells: $35.5 \pm 9.3 \%$ in Protocol A and $43.4 \pm 17.4 \%$ in Protocol B compared to $48.2 \pm 16.3 \%$ after no incubation (not statistically significant, Figure 5.3C).

While pre-incubation with the cryoprotectant appeared to enhance cryopreservation of whole islets, longer equilibration period with DMSO appeared to result in decrease in viability of dissociated (single) islet cells. This is likely because each single islet cell has direct exposure to DMSO in the solution and requires a shorter time to equilibrate with the cryoprotectant than whole islets. The prolonged incubation, therefore, results in overexposure to DMSO and toxic effects.

In conclusion, equilibration with DMSO is beneficial in cryopreservation of whole human pancreatic islets and does not decrease viability in mouse islet cryopreservation. However, it has detrimental effect if islets are dissociated into single cells and cryopreserved. All subsequent experiments with whole pancreatic islets were therefore performed using the step-wise addition with equilibration protocol (Protocol A) as described by Rajotte [190]. 
Subsequent experiments with dissociated islet cells were performed using single step addition of 2 M DMSO followed by immediate freezing (Protocol C).

\subsubsection{Freezing rate}

Mazur's two-factor hypothesis [35] suggests that injury to cells during freezing occurs either due to dehydration (if cooling is too slow) or intracellular ice formation (if cooling is too rapid). Optimal freezing rate for a specific cell type balances these two processes in order to minimise freezing damage. In pancreatic islets, the optimal slow freezing rate was found to be -0.25 or $-0.3{ }^{\circ} \mathrm{C} / \mathrm{min}[74,191]$. While islets can also tolerate rapid cooling rates of over $-70{ }^{\circ} \mathrm{C} / \mathrm{min}$ [191], slow-freezing was used in all experiments.

Two different methods of achieving specific cooling rates were compared: using a programmable freezer and a conventional freezing container (Mr. Frosty ${ }^{\mathrm{TM}}$ ). While freezing containers cannot achieve cooling rates as slow as $-0.3{ }^{\circ} \mathrm{C} / \mathrm{min}$, their use may be advantageous due to the fact that programmable freezers are costly and not widely available across research and clinical laboratories. The following two freezing protocols for mouse and human islets were therefore tested: Firstly, a controlled-rate freezer was used to achieve the freezing rate of $-0.3^{\circ} \mathrm{C} / \mathrm{min}$ (Controlled freezing), and secondly, a Mr. Frosty ${ }^{\mathrm{TM}}$ freezing container placed at $-80^{\circ} \mathrm{C}$ freezer was used to simulate higher freezing rate (approximately $-1{ }^{\circ} \mathrm{C} / \mathrm{min}$ ) in an uncontrolled manner (Uncontrolled freezing). The latter protocol is identical to that used for freezing mononuclear cells in Chapter 4.

\section{Mouse pancreatic islets}

There were significant differences in the viability of cryopreserved mouse pancreatic islets depending on the freezing rate used $(p<0.01$ on Day $0, p<0.0001$ on Day $3, p=0.001$ on Day 6, Figure 5.4A). The viability of islets frozen by the Controlled method did not decrease below $70 \%$ on Days 0,3 and 6 while the Uncontrolled samples were $30.6 \pm 7.8 \%$ viable immediately after thawing and less than $17 \%$ viable on following days. Although the variability in size of islets was large, islets frozen using controlled-rate freezer were on average larger than those from freezing container (Figure 5.4B).

\section{Human pancreatic islets}

In human islets, the viability of islets from both protocols was identical on all the days measurement was taken (Figure 5.4C). There was a trend of larger islets surviving when the Controlled protocol was used, however these differences were not statistically significant (Figure 5.4D). 

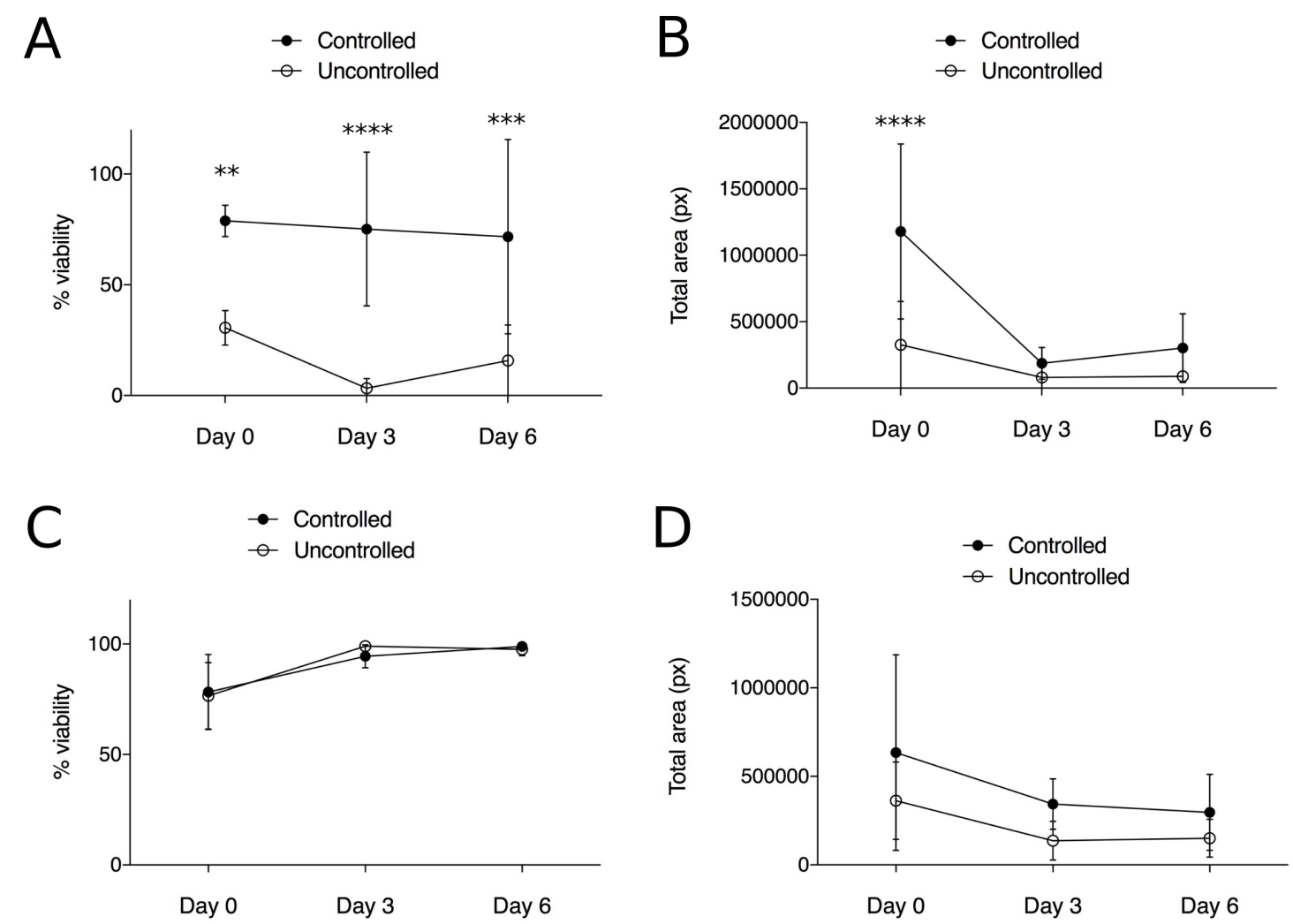

Fig. 5.4 Comparison of controlled and uncontrolled freezing for mouse and human pancreatic islets from Day 0 to Day 6 after thawing. (A) Viability of mouse pancreatic islets frozen by controlled-rate freezer (Controlled) vs. freezing container (Uncontrolled). (B) Size of thawed mouse pancreatic islets. (C) Viability of human pancreatic islets frozen by controlled-rate freezer (Controlled) vs. freezing container (Uncontrolled). (D) Size of thawed human pancreatic islets. Average \pm SD viability/size of each experimental sample is shown, calculated from viability of $n=6-10$ mouse islets or $\mathrm{n}=4-21$ human islets. Statistical comparison was done using two-way ANOVA followed by Sidak's correction, significant differences are marked by asterisks: $* \mathrm{p} \leq 0.05, * * \mathrm{p} \leq 0.01$; $* * * \mathrm{p} \leq 0.001, * * * * \mathrm{p} \leq 0.0001$. 
This data suggests that slow cooling rate of $-0.3{ }^{\circ} \mathrm{C} / \mathrm{min}$ achieved by a controlled-rate freezer is superior to uncontrolled freezing at the rate of $-1{ }^{\circ} \mathrm{C} / \mathrm{min}$ in both mouse and human islets. This is in agreement with a previous study conducted by Rajotte et al. who tested the same freezing rates and observed that islets frozen at rate of $-0.3{ }^{\circ} \mathrm{C} / \mathrm{min}$ we able to reverse diabetes in a rat model while islets frozen at $-1{ }^{\circ} \mathrm{C} / \mathrm{min}$ were not [74].

In the experiments presented here, the difference between the two methods has been less pronounced in human islets than in mouse islets. Rajotte et al. compared cryopreservation of rat and canine pancreatic islets and, noting different outcomes, suggested that differences in tissue of small (mouse, hamster, rat) and large (dog, human) species may call for different parameters of cryopreservation, including freezing rate [192]. It is therefore possible that human islets can tolerate wider range of cooling rates than mouse islets.

To conclude, my results suggest that the use of programmable freezers to achieve slow cooling rates for islet cryopreservation is necessary to preserve their viability and size, both of which contribute to the functional pancreatic islet mass available for transplantation. All subsequent experiments were therefore conducted using a programmable freezer at the rate of $-0.3{ }^{\circ} \mathrm{C} / \mathrm{min}$.

\subsubsection{DMSO removal protocol}

Post-thaw removal of DMSO is a necessary step of the cryopreservation protocol ensuring that the high toxic concentrations are removed and cells are brought back into a physiological medium. This must be done in a manner that reduces the osmotic shock caused by the rapid change in solution osmolarity. The cryoprotectants are therefore ideally diluted post-thaw in a step-wise fashion to allow the cells to adjust to the new concentration of solutes without risking damage. In single cell cryopreservation, drop-wise addition of thawing medium is often recommended to prevent the osmotic shock [193, 194].

In islet cryopreservation, several approaches are used to ensure optimal transfer of islets from the cryoprotectant medium into the culture medium. The most widely used protocol utilises $0.75 \mathrm{M}$ sucrose which is exchanged for the $2 \mathrm{M}$ DMSO immediately after thawing. This dilutes the DMSO down to concentrations which have low toxicity while preventing osmotic shock to the islet cells. Sucrose is then diluted in several steps before the islets are brought back to normal culture medium. This approach has been suggested by Rajotte and Mazur and remains the standard method in islet cryopreservation [193]. An alternative approach suggested by Lakey et al. recommends slow dilution of DMSO with culture medium without addition of sucrose [87].

Three different DMSO removal protocols were tested in parallel to identify the optimal method to thaw both mouse and human islets (schematic representation in Figure 5.5). The 


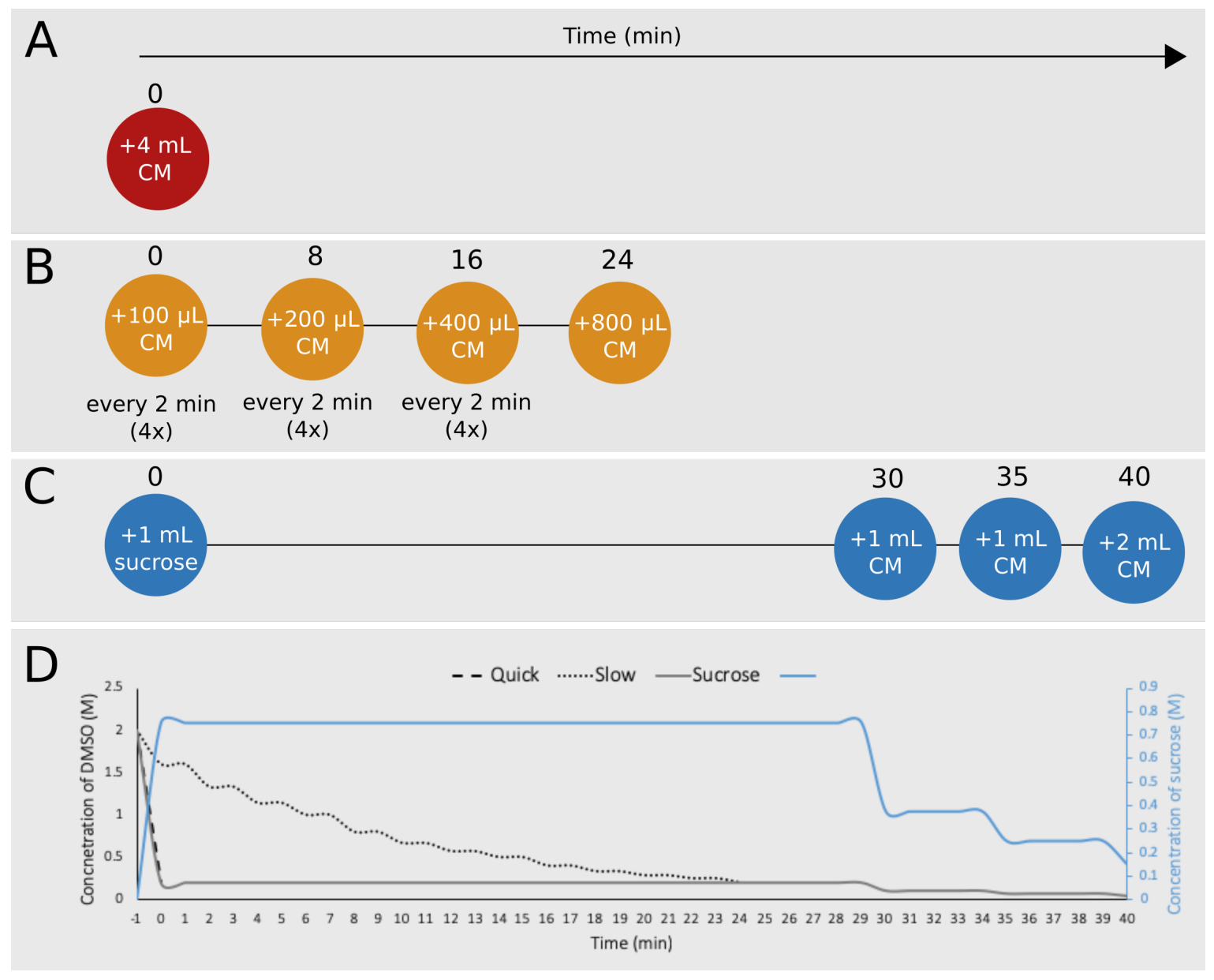

Fig. 5.5 Design of the islet cryoprotectant removal protocol study. Time 0 represents the time when the last small ice crystal is observed after rapid thawing in a water bath. Numbers above colourful circles represent time in minutes from this time point. Each circle represents an addition of culture medium (CM) or $0.75 \mathrm{M}$ sucrose to the thawed islets. (A) Schematic of QUICK thawing protocol: $10 \times$ dilution of DMSO in a single step. (B) Schematic of the SLOW thawing protocol: step-wise dilution of DMSO using CM over 24 min until $10 \times$ dilution is reached. (C) Schematic of the SUCROSE thawing protocol - at time 0 , DMSO is exchanged for $0.75 \mathrm{M}$ sucrose and incubated on ice for $30 \mathrm{~min}$, followed by step-wise dilution of sucrose. (D) Model of changes of concentrations in DMSO and sucrose over time using the three different cryoprotectant removal protocols. Dotted and dashed lines show the decreasing concentration of DMSO (left y axis) while the solid line shows the concentration of sucrose in SUCROSE protocol (right y axis). 
first protocol, referred to as 'QUICK', contains a single-step $10 \times$ dilution of the cryopreservation medium with islets. The second protocol, 'SLOW', is the step-wise dilution with culture medium described by Lakey et al. [87]. The third protocol, 'SUCROSE', is the protocol designed by Rajotte et al. [193] which involves the exchange of DMSO for sucrose. In addition, the QUICK and SLOW protocols were tested in cryopreservation of dissociated mouse pancreatic islet cells to examine whether the use of step-wise protocols benefits both single cells and cell clusters.

\section{Mouse pancreatic islets}

In cryopreservation of mouse islets, the highest viability immediately after thawing and 3 days post-thaw was achieved using the SLOW protocol (74.4 $\pm 9.2 \%$; Figure 5.6A). The SLOW protocol also better preserved their morphology, including size and fibrous islet capsule surrounding the cells (observed as a thin dark line around the islets; Figure 5.6B). The QUICK protocol resulted in a decreased post-thaw viability $(54.4 \pm 25.2 \%)$ which remained low even after three days of culture. The morphology of islets was also changed; the cell clusters were no longer surrounded by the fibrous islet capsule with the exception of few very small islet-like clusters which are surrounded by it. Islets thawed using the SUCROSE protocol had decreased viability immediately post-thaw (46.6 $\pm 16.9 \%$ ), which recovered marginally after three days of culture. Moreover, they lost a large portion of their volume and high proportions of small fragments were observed (Figures 5.6A and B).

\section{Human pancreatic islets}

The experiments were repeated using human islets. A fourth protocol was added in the human islet thawing, to address the increased amount of debris observed in the SUCROSE protocol. The modification involved pouring the cell suspension instead of pipetting and handpicking the islets instead of centrifuging to minimise any mechanical stress (MODIFIED SUCROSE protocol).

The viability of islets thawed by the QUICK method was again decreased $(52.0 \pm 0.2 \%)$ and did not recover over time (Figure 5.6C). The viability in the rest of the conditions was similar to the QUICK protocol immediately after thawing but seemed to recover in culture over time. There was no observable difference in viability between the SLOW, SUCROSE and MODIFIED SUCROSE protocol. When comparing the SUCROSE and MODIFIED SUCROSE protocols, more fragments were found in the SUCROSE protocol (Figure 5.6D). 
A

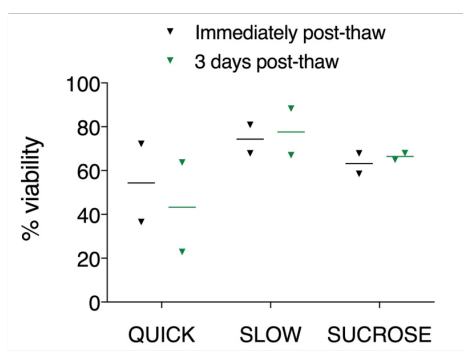

C

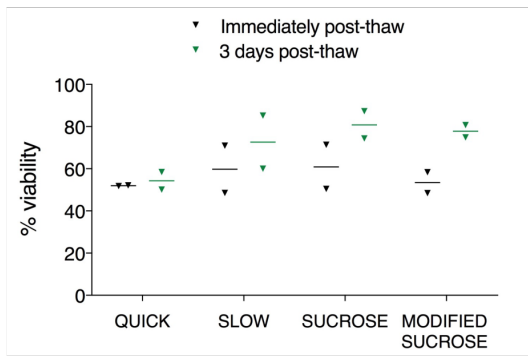

QUICK

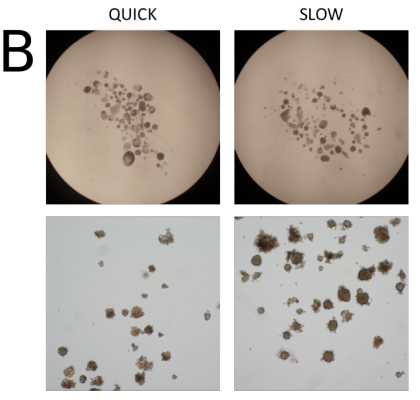

SUCROSE

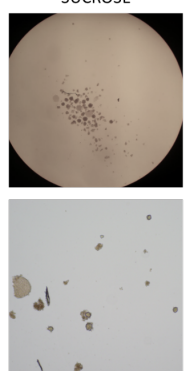

$\mathrm{D}$

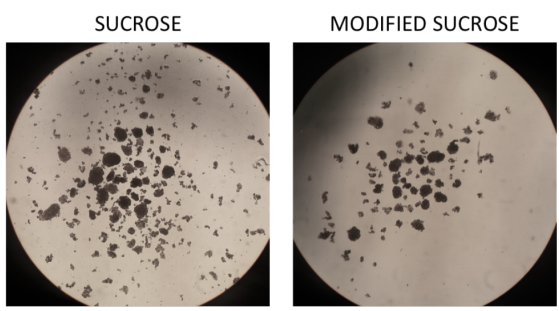

$\mathrm{E}$

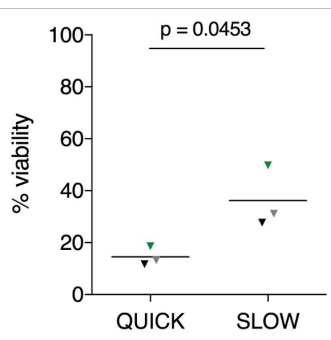

$\mathrm{F}$

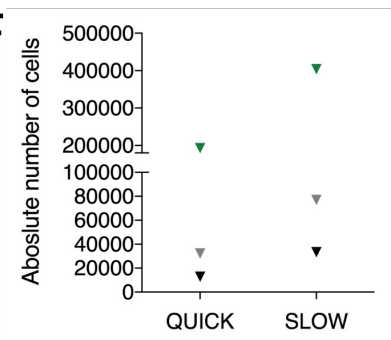

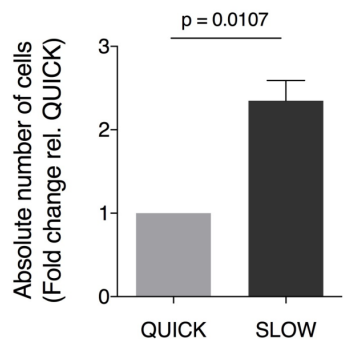

Fig. 5.6 Comparison of thawing protocols for mouse and human pancreatic islets. (A) Post-thaw viability of mouse pancreatic islets thawed using three different protocols immediately after thawing and 3 days after thawing $(n=2)$. Each triangle represents the average viability of one experimental sample calculated from 6-14 islets; horizontal line represents the mean. (B) Bright field images of mouse pancreatic islets thawed using three different protocols. Top row shows all islets recovered after thawing, bottom row show the same islets under magnification. (C) Post-thaw viability of human pancreatic islets thawed using four different protocols immediately after thawing and 3 days after thawing $(n=2)$. Each triangle represents the average viability of one experimental sample calculated from 5- 17 islets; horizontal line represents the mean. (D) Bright field images of human pancreatic islets thawed using sucrose protocol and its modification. All recovered islets shown. (E) Post-thaw viability of dissociated mouse pancreatic islets $(\mathrm{n}=3)$. $(\mathbf{F})$ Post-thaw absolute cell count of dissociated islet cells (left) and fold-change relative to the absolute cell count in QUICK protocol (right; $n=3$ ). Each triangle represents viability of one sample, matching samples from one experiment are shown in the same colour. Statistical comparison was done using paired t-test, statistically significant p-values are specified in each graph. 


\section{Dissociated islet cells}

For dissociated islet cells, the SLOW protocol resulted in significantly higher viability than the QUICK protocol $(36.2 \pm 11.9 \%$ versus $14.5 \pm 3.6 \%$; $t$-test, $\mathrm{p}=0.0453$, Figure $5.6 \mathrm{E})$. The absolute cell yield after cryopreservation was also higher ( $2.3 \pm 0.2$ times) using the SLOW thawing protocol (Figure 5.6F).

Based on these findings, optimal DMSO removal protocols were selected for mouse and human islets to be used in all following cryopreservation experiments. In mouse islets, the SLOW protocol was superior to the other protocols both in viability and morphology. In human islets, the SLOW and SUCROSE protocols appeared to preserve viability equally well. In studies which compared these two protocols, SLOW protocol was equally good [74] or better [87] than SUCROSE protocol. The current results are therefore in agreement with these studies. The protocols, however, have not been previously compared using human islets and it appears as there are some inter-species differences. Slow dilution of DMSO was also beneficial in dissociated islet cell thawing, suggesting that single cells and cell clusters are equally sensitive to osmotic stress.

Lakey et al. [87] suggested that the decreased cryosurvival after addition of $0.75 \mathrm{M}$ sucrose during thawing may be caused either by osmotic stress from the sucrose or centrifugation step which could lead to fragmentation of islets. The tested MODIFIED SUCROSE protocol indeed resulted in decreased amount of debris in the post-thaw suspension. However, this may have been caused by omitting the small islet fragments during manual handpicking rather than the debris not being present after thawing. These modifications are used in all future experiments to avoid possible negative effects of dead cell presence in post-thaw culture of islets.

In summary, based on the current data and the evidence from the literature, the SLOW protocol was used for subsequent experiments with whole and dissociated mouse islets. Since the SUCROSE protocol is the standard in the field of human islet cryopreservation, it was used in all following human islet cryopreservation experiments with the modifications we introduced to decrease the mechanical stress to the islets (i. e. MODIFIED SUCROSE protocol). 


\subsection{Trehalose as a sole cryoprotectant for islets}

\subsubsection{Trehalose concentration}

With the DMSO protocol optimised, efficacy of trehalose for cryopreservation of pancreatic islets was tested. In the first instance, trehalose was used on its own to investigate its potential as a sole cryoprotectant for pancreatic islets.

In the first set of experiments, mouse pancreatic islets were cryopreserved in different concentrations of trehalose (without any incubation) in the controlled-rate freezer and thawed using the SLOW thawing protocol. Viability of islets was evaluated using FDA/PI staining immediately post-thaw.

The post-thaw viability of all islets cryopreserved using trehalose alone was very low compared to the DMSO control (0.06 - 8.79\% viability for trehalose, Figure 5.7A). While there was a considerable variability between the three replicates, $200 \mathrm{mM}$ trehalose performed the best in all three repeats of the experiment but nonetheless provided minimal protection. Viability was not measured again at a later time point ( $24 \mathrm{~h}$ post-thaw), as all the remaining islets in the trehalose groups died and disintegrated during this period. Trehalose alone, without any incubation, therefore is not an effective cryoprotectant for islets.

\subsubsection{Trehalose incubation time}

Because islets are cell clusters, diffusion of cryoprotectant to all the cells naturally takes time. Diffusion of small molecules was investigated in Section 3.4 of Chapter 3. The findings proposed that it takes $6 \mathrm{~h}$ at $37^{\circ} \mathrm{C}$ for a dye to equilibrate in all cells of the islet. Trehalose, being also a small molecule travelling in an aqueous solution, may be expected to require a similar time to diffuse throughout the cluster. As shown in Section 5.2.2 of this Chapter, prolonged incubation of mouse islets with $200 \mathrm{mM}$ trehalose did not have a negative effect on their viability. It was hypothesised, therefore, that incubation time before cryopreservation could increase the viability of trehalose-cryopreserved islets.

\section{Mouse islets}

The hypothesis was first tested using mouse pancreatic islets which were incubated with $200 \mathrm{mM}$ trehalose for $15 \mathrm{~min}$ to $48 \mathrm{~h}$ and then cryopreserved using the programmable freezer. After thawing using the SLOW protocol, viability was measured immediately. Control DMSO islets were cryopreserved along with the trehalose samples.

Again, viability of islets in all samples where trehalose was the only cryoprotectant was extremely low and did not in these two experiments exceed $2.95 \%$ in any of the conditions 

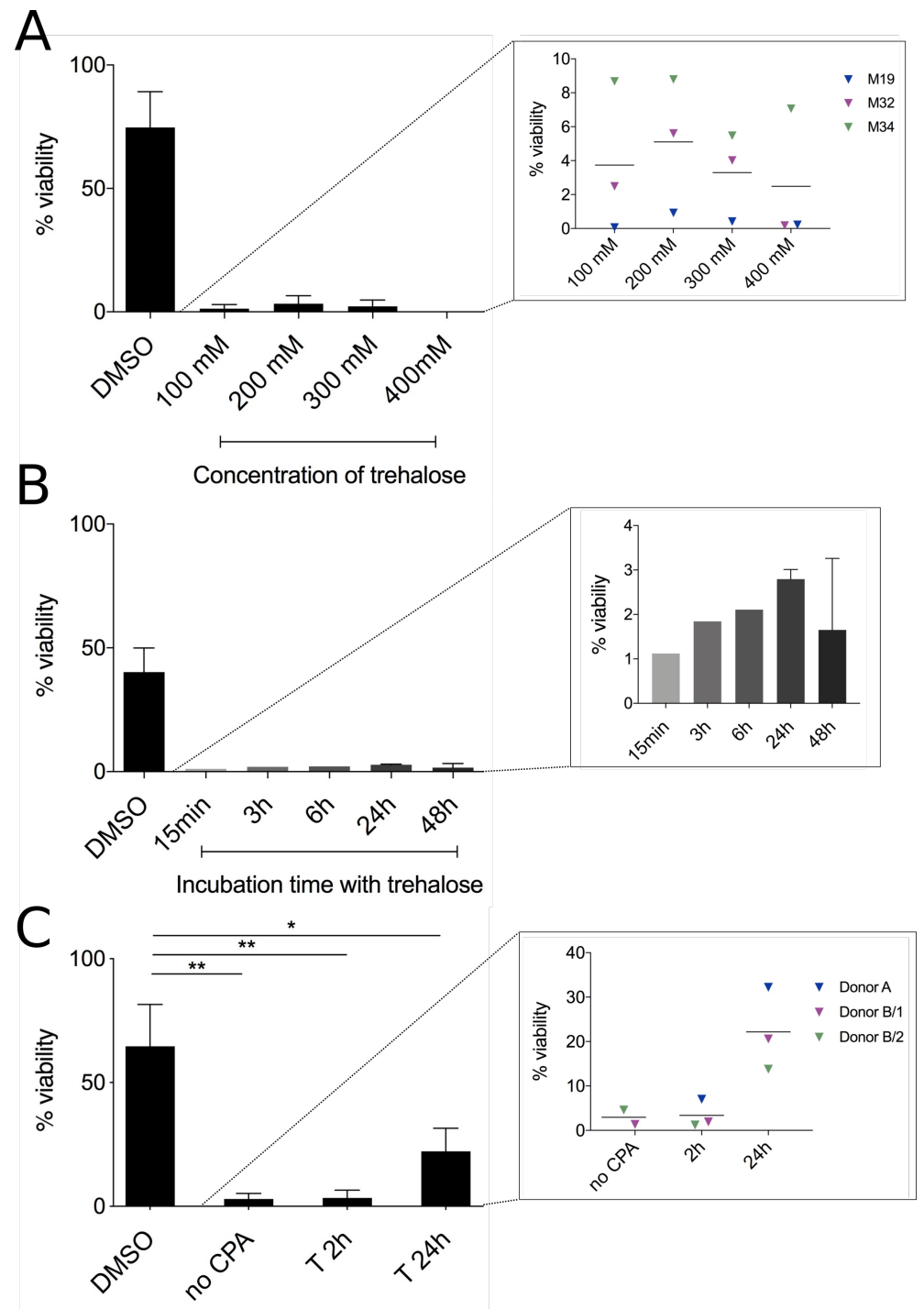

Fig. 5.7 Cryopreservation with trehalose only: concentrations and incubation times. (A) Post-thaw viability of mouse pancreatic islets cryopreserved with DMSO or different trehalose concentrations (100 $400 \mathrm{mM}$ ). Viabilities of the individual replicates (M19, M32 and M34) of trehalose-cryopreserved islets are shown in the zoomed graph on the right. Bars represent mean $\pm S D, n=3$. (B) Post-thaw viability of mouse pancreatic islets cryopreserved with DMSO (no extra incubation) or $200 \mathrm{mM}$ trehalose ( $15 \mathrm{~min}, 3 \mathrm{~h}, 6 \mathrm{~h}, 24 \mathrm{~h}$ or $48 \mathrm{~h}$ incubation prior to freezing). Viabilities of the trehalosecryopreserved islets are shown in the zoomed graph on the right. Bars represent mean $\pm \mathrm{SD}, \mathrm{n}=1-2$. (C) Post-thaw viability of human pancreatic islets cryopreserved with DMSO (no extra incubation), no cryoprotectant or $200 \mathrm{mM}$ trehalose ( $2 \mathrm{~h}$ or $24 \mathrm{~h}$ incubation prior to freezing). Viabilities of the individual replicates (Donor A or Donor B replicates 1 and 2) of trehalose-cryopreserved islets are shown in the zoomed graph on the right. Bars represent mean $\pm \mathrm{SD}, \mathrm{n}=3$. Triangles represent average viabilities from each replicate calculated from $4-17$ mouse islets or $2-14$ human islets, triangles of same colour represent results from the same experiment, horizontal lines represent mean. Differences in (C) were compared using mixed effects one-way ANOVA followed by Tukey's HSD post-hoc test. Statistically significant differences are marked by asterisks: $* \mathrm{p} \leq 0.05, * * \mathrm{p} \leq 0.01$. 
(Figure 5.7B). Highest viability in both experiments was measured for the incubation time of $24 \mathrm{~h}$.

Because the number of replicates was low, no statistical comparisons were made but there seemed to be a trend of viability increasing with incubation time, peaking at $24 \mathrm{~h}$ of incubation.

\section{Human islets}

The experiments were repeated using human islets, but the number of trehalose conditions was reduced due to the shortage of tissue to $2 \mathrm{~h}$ and $24 \mathrm{~h}$ only. DMSO and no cryoprotectant (no CPA) controls were included. Islets from two donors were used and the experiment was repeated twice for the second donor. Thawing was performed using MODIFIED SUCROSE protocol and viability measured immediately post-thaw.

Although not statistically significant, there is a clear trend to higher survival of pancreatic islets at $24 \mathrm{~h}$ incubation time, compared to $2 \mathrm{~h}$ incubation $(22.2 \pm 9.3 \%$ vs. $3.4 \pm 3.2 \%, \mathrm{p}=0.2444$; Figure 5.7C). Both of these were still significantly lower than DMSO-cryopreserved islets $(64.6 \pm 16.9 \%, \mathrm{p}=0.0159$ and $\mathrm{p}=0.0032$, respectively).

The $2 \mathrm{~h}$ incubation of human islets with trehalose provided similar result to cryopreservation without any cryoprotectant, confirming that trehalose cannot be used as a sole cryoprotectant without sufficient incubation. Data from mouse islets suggest the same, although repeating the experiment would provide more conclusive result. However, even without additional repeats it is clear that even long incubation times do not increase the efficacy of trehalose to provide results comparable to the standard DMSO protocol.

The viability of human islets cryopreserved with trehalose only and incubated for $24 \mathrm{~h}$ was higher than the one measured for mouse pancreatic islets. One potential explanation may be that the human islets generally have worse quality after isolation and before the cryopreservation than mouse islets. The edges of human islets are not smooth and the fibrous capsule may be damaged, giving easier access of trehalose to all the cells. Human islets may additionally be more resistant to cryoinjury.

Cryopreservation with trehalose on its own could not be compared to any results from the literature because similar experiments have never been published. It is possible that trehalose performance in these experiments could be improved by its intracellular loading. Preliminary experiments on the addition of PP-50 and PP-75 polymers were performed and the results are shown in Appendix C. Briefly, the addition of the polymers did not show any improvement over using trehalose without intracellular loading at $2 \mathrm{~h}$ and $24 \mathrm{~h}$, regardless of the polymer type. This is in agreement with data obtained from cryopreservation of splenocytes with trehalose and the polymers in Chapter 4. 
To conclude, a range of trehalose concentrations and incubation times were tested for mouse and human pancreatic islets but none of the conditions provided protection comparable to the current DMSO standard. The presented data, therefore, confirm that trehalose alone cannot be used instead of DMSO for cryopreservation of pancreatic islets.

\subsection{Addition of trehalose to DMSO protocol}

Because trehalose is not an effective cryoprotectant on its own, the next approach tested in this project was its addition to the standard DMSO protocol. While trehalose combined with DMSO was tested in pancreatic islets previously by Beattie et al. [83], this approach has never been tested systematically or subsequently reported.

\subsubsection{Effect of pre-incubation with trehalose}

The evidence from diffusion experiments in Chapter 3 and even from Section 5.4 suggests that incubation time of $6-24 \mathrm{~h}$ may be needed for trehalose to fully permeate the whole volume of pancreatic islets. However, because of toxicity, such long incubation times with DMSO would not be possible. A new protocol was therefore designed which includes an incubation time with trehalose only, subsequently supplemented with DMSO as per the standard protocol.

\section{Incubation time}

Mouse islets were pre-incubated with $200 \mathrm{mM}$ trehalose for $1-48 \mathrm{~h}$ at $37^{\circ} \mathrm{C}$. $45 \mathrm{~min}$ before the end of this incubation time, the DMSO was added using the previously described protocol (see DMSO A protocol in Section 5.3.1), while keeping the trehalose concentration constant at $200 \mathrm{mM}$ (the added DMSO solutions contain $200 \mathrm{mM}$ trehalose). All samples were then cryopreserved in the programmable freezer, thawed and assessed for viability immediately post-thaw and $24 \mathrm{~h}$ post-thaw.

Immediately after thawing, the viability of DMSO + trehalose (DT) samples was not significantly different from DMSO-cryopreserved samples, regardless of the incubation time (Figure 5.8). At $24 \mathrm{~h}$ after thawing, samples incubated with trehalose for $1 \mathrm{~h}$ or $3 \mathrm{~h}$ again did not show significantly different survival. However, there was a significant improvement of viability of the sample pre-incubated with trehalose for $6 \mathrm{~h}$ (DT 6h) compared to DMSO $(\mathrm{p}=0.0402)$. Longer incubation times $(24 \mathrm{~h}$ and $48 \mathrm{~h})$ did not perform better than the $6 \mathrm{~h}$ incubation at $24 \mathrm{~h}$ after thawing and there was a significant decrease in viability for the $48 \mathrm{~h}$ incubation when compared to $6 \mathrm{~h}$. 


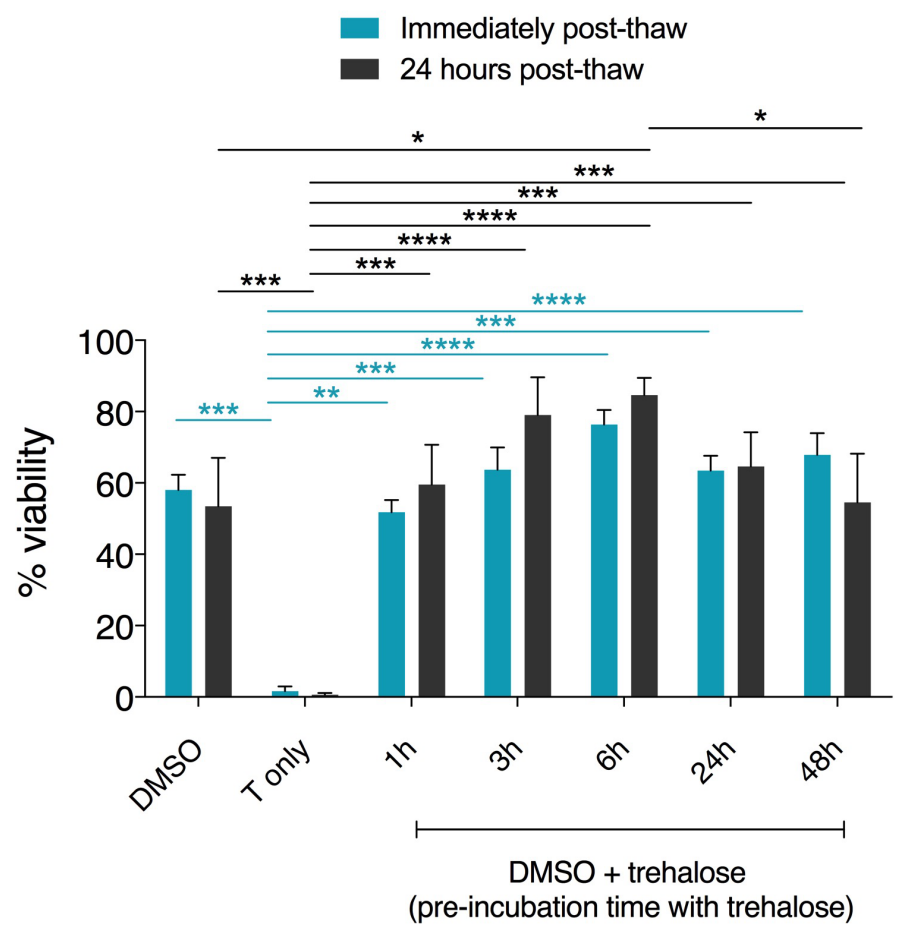

Fig. 5.8 Supplementation of the DMSO protocol with trehalose. Post-thaw viability of mouse pancreatic islets pre-incubated with $200 \mathrm{mM}$ trehalose for $1-48 \mathrm{~h}$ and cryopreserved in $2 \mathrm{M}$ DMSO $+200 \mathrm{mM}$ trehalose along with fresh, $2 \mathrm{M}$ DMSO only and $200 \mathrm{mM}$ trehalose only controls. Viability measured immediately post-thaw (light blue bars) and $24 \mathrm{~h}$ post-thaw (dark blue bars) shown. Bars represent mean $\pm \mathrm{SD}, \mathrm{n}=3$ (each measurement calculated from $5-35$ islets), repeated measures two-way repeated measures ANOVA followed by Tukey's HSD post-hoc test, significant differences are marked by asterisks: $* \mathrm{p} \leq 0.05, * * \mathrm{p} \leq 0.01 ; * * * \mathrm{p} \leq 0.001, * * * * \mathrm{p} \leq 0.0001$. T only $=$ trehalose only

These experiments suggest that trehalose can improve DMSO-based cryopreservation if the pre-incubation period is sufficiently long. In this case, the appropriate incubation time was $6 \mathrm{~h}$. This correlates well with the findings from the diffusion experiments in Chapter 3.4 which showed that it takes $6 \mathrm{~h}$ for a dye to equilibrate in the whole volume of pancreatic islets.

\section{Survival of cell clusters}

In the above experiments, immediately after thawing, each islet contained live and dead cells in a proportion close to the average viability. The viabilities of all individual islets within a condition had normal distribution (Figure 5.9A). Surprisingly, this was not true at $24 \mathrm{~h}$ after thawing. All imaged islets either contained mostly live cells while the remaining islets were 
almost fully dead (Figure 5.9B). There was a trend of higher proportion of live islets in the DT6h group compared to DMSO alone at $24 \mathrm{~h}$.

To investigate the time course of this phenomenon, an experiment was designed where the viability of the thawed islets was measured immediately post-thaw and then at $3 \mathrm{~h}, 6 \mathrm{~h}$, $12 \mathrm{~h}$ and $24 \mathrm{~h}$ after thawing. The data suggest that predominantly dead islets can be detected as early as $3 \mathrm{~h}$ post-thaw (Figure 5.9C).

The distribution of viability data $24 \mathrm{~h}$ after thawing was unexpected and raised additional questions regarding survival of pancreatic islets. Viability in other cell types is always viewed as a characteristic of each single cell but from these experiments it appears as if viability of cells in a cluster is influenced not only by the status of the individual cells but the overall health of the cluster or signals from the neighbouring cells. One potential mechanism may be related to the need for a critical amount of cell-cell contact or communication between adjacent cells. Pancreatic islet cells do not survive well when dissociated into single cells (data not shown) and cell-cell contact or short-distance paracrine signalling could therefore play a critical role in their survival. It is possible, therefore, that cell death within the cluster results in a progressive loss of these trophic signals to adjacent cells. If a threshold of cell death or injury is exceeded, the loss of trophic signals will lead to death of adjacent cells, ultimately leading to demise of the whole islet. Conversely, islets containing mostly alive cells may have mechanisms which enable the cluster to exclude the dead cells, leaving the islet with predominantly live cells (Figure 5.9D).

This proposed mechanism does not necessarily need to be an active process. For example, re-establishing cell-cell contact with live (but not dead) cells may gradually result in the dead cells moving to the periphery and finally being excluded from the main cluster. The underlying mechanisms, and the biological relevance of this process has not yet been explored. Exclusion of dead cells from the cluster necessarily results in reduction of the islet size/mass. This is very difficult to measure due to the $3 \mathrm{D}$ structure of islets and would require real-time prolonged imaging of individual islets.

The distribution of data at $24 \mathrm{~h}$ after thawing has an important implication for their statistical analysis. Because the distribution of the data is bimodal, standard statistical parameters such as central tendency or variability could be inaccurate using methods developed for normally distributed data. Means and standard deviations shown in Figure 5.8A are, therefore, not fully applicable but are nonetheless displayed to show the trend which can be observed but not yet quantified. Appropriate statistical methods to analyse this data are complex and have not yet been identified. 


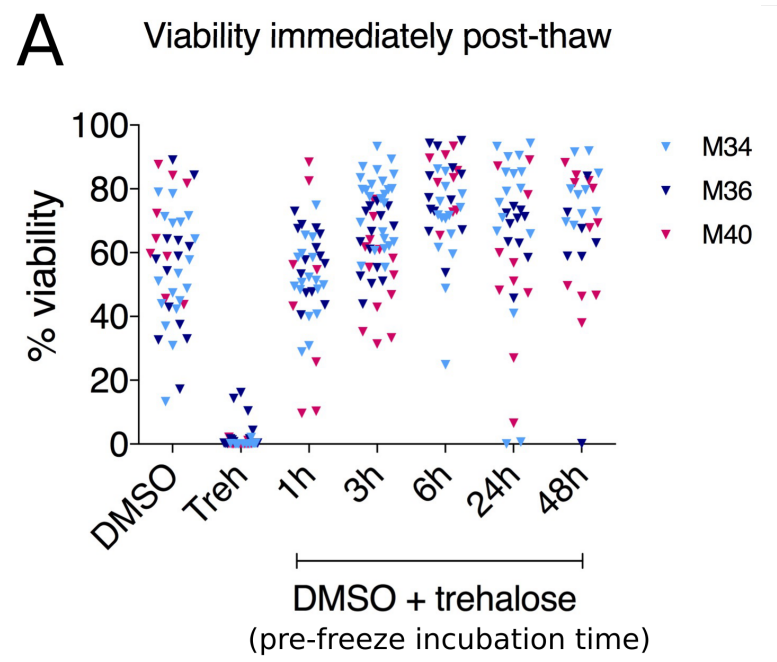

B Viability 24 hours post-thaw
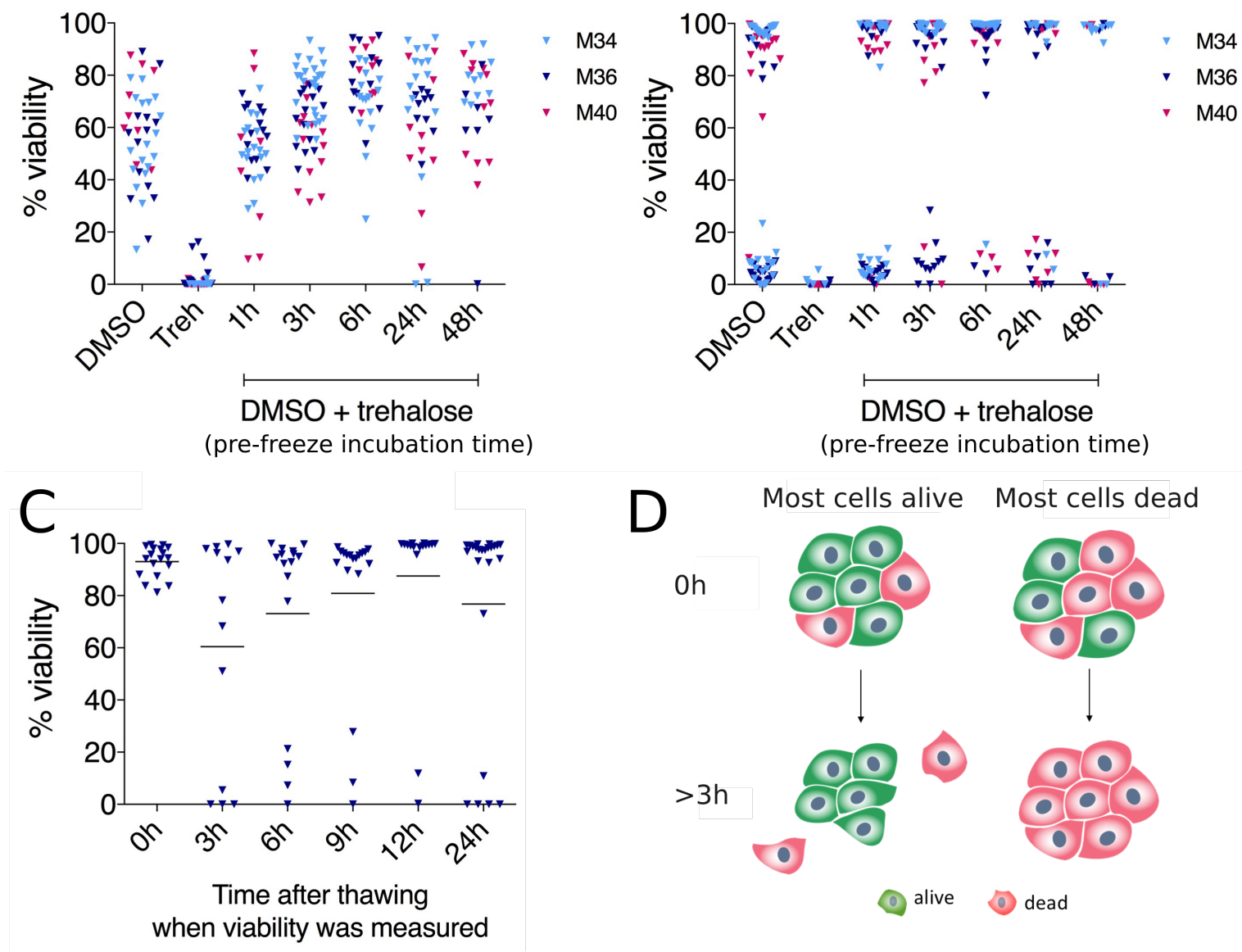

D Most cells alive Most cells dead

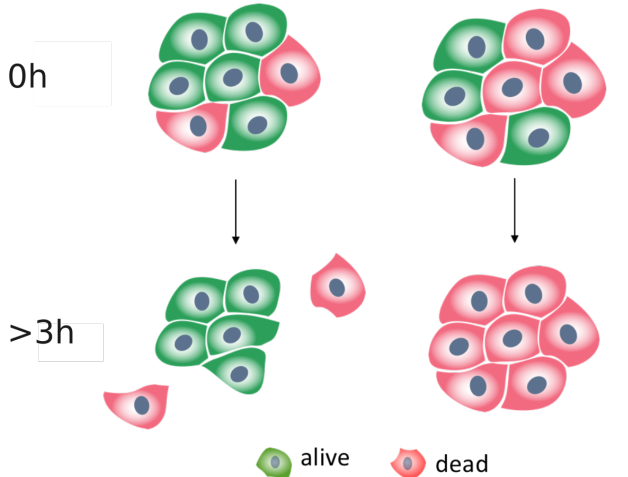

Fig. 5.9 New insight into the mechanism of survival of cell clusters. (A) Post-thaw viability of individual islets cryopreserved with DMSO only, trehalose only or combination of DMSO and trehalose (with varying trehalose incubation time) measured immediately post-thaw. (B) Post-thaw viability of individual islets from the experiments shown in (A) measured 24 h post-thaw. (C) Viability of individual islets cryopreserved with DT6h and measured immediately post-thaw and $3 \mathrm{~h}, 6 \mathrm{~h}, 9 \mathrm{~h}$, $12 \mathrm{~h}$ and $24 \mathrm{~h}$ post-thaw. For (A)-(C), no statistical analysis was performed. Triangles represent individual islet viabilities, triangles of same colour represent results from the same experiment. DT6h = DMSO + trehalose pre-incubated for $6 \mathrm{~h}$; Treh = trehalose. (D) Schematic of the proposed cell cluster fate based on the viability immediately post-thaw. Left: If majority of cells within the cluster remained alive immediately after thawing, the islet excludes the minority of dead cells, resulting in a smaller but fully viable islet when viability is measured at a later time point. Right: If large amount of cells in the islet are already dead immediately after thawing, sufficient support is not provided for the remaining live cells which results in their cell death. 


\subsubsection{Concentrations of components}

Combining multiple cryoprotectants has been previously suggested as a way to decrease the concentration of the individual (often toxic) cryoprotectants. I therefore next explored whether lowering the concentration of DMSO or trehalose in the new DT6h protocol could provide enhanced protection by decreasing the osmotic stress or toxic effects of the high cryoprotectant concentrations. Higher concentrations have also been explored to see if these could provide additional protection.

\section{Concentration of trehalose}

In the first set of experiments, mouse pancreatic islets were cryopreserved using $100-300 \mathrm{mM}$ trehalose concentration in the same manner as the DT6h protocol $(6 \mathrm{~h}$ incubation with the correct trehalose concentration, followed by addition of DMSO over $45 \mathrm{~min}$ ). DMSO concentration in all conditions was kept at $2 \mathrm{M}$. Viability of thawed islets was assessed immediately post-thaw and $24 \mathrm{~h}$ post-thaw.

The results from this experiment demonstrated that increasing the trehalose concentration in the DT6h protocol above $200 \mathrm{mM}$ led to a decrease in post-thaw viability compared to fresh islets ( $\mathrm{p}=0.0089$ for $300 \mathrm{mM}$ trehalose; Figure 5.10A). On the other hand, decreasing the trehalose concentration did not lead to worse cryoprotection $(96.61 \pm 2.01 \%$ viability in $200 \mathrm{mM}$ trehalose vs. $92.59 \pm 7.8 \%$ viability in $100 \mathrm{mM}$ trehalose; $\mathrm{p}=0.9976$ ).

Because the $50 \%$ decrease in trehalose concentration did not have detrimental effect on islet viability, a second set of experiments was performed with the same design but lowering the trehalose concentration from $200 \mathrm{mM}$ down to $25 \mathrm{mM}$.

In this experiment, the average viabilities were approximately the same in all four trehalose concentrations (25, 50, 100 and $200 \mathrm{mM}$; Figure 5.10B). Again, additional decrease in trehalose concentration did not seem to negatively affect the islet viability and still resulted in statistically significant increase in viability compared to DMSO alone $(0 \mathrm{mM}$ concentration of trehalose in Figure 5.10B). Conversely, the islets cryopreserved in lower trehalose concentration ( $25 \mathrm{mM}$ and $50 \mathrm{mM}$ ) were larger in size and more similar to fresh islets in their morphology when observed under microscope (data not shown). A lower concentration of trehalose may, therefore, result in enhanced cryoprotection.

The decrease in viability for islets cryopreserved with $300 \mathrm{mM}$ trehalose could be due to a direct toxic effect of trehalose itself, or due to exceeding the islet tolerance for combined osmotic pressure of DMSO and trehalose. From the available data it appears that the lowest concentration at which trehalose exhibits its protective effect is much lower than in DMSO- 

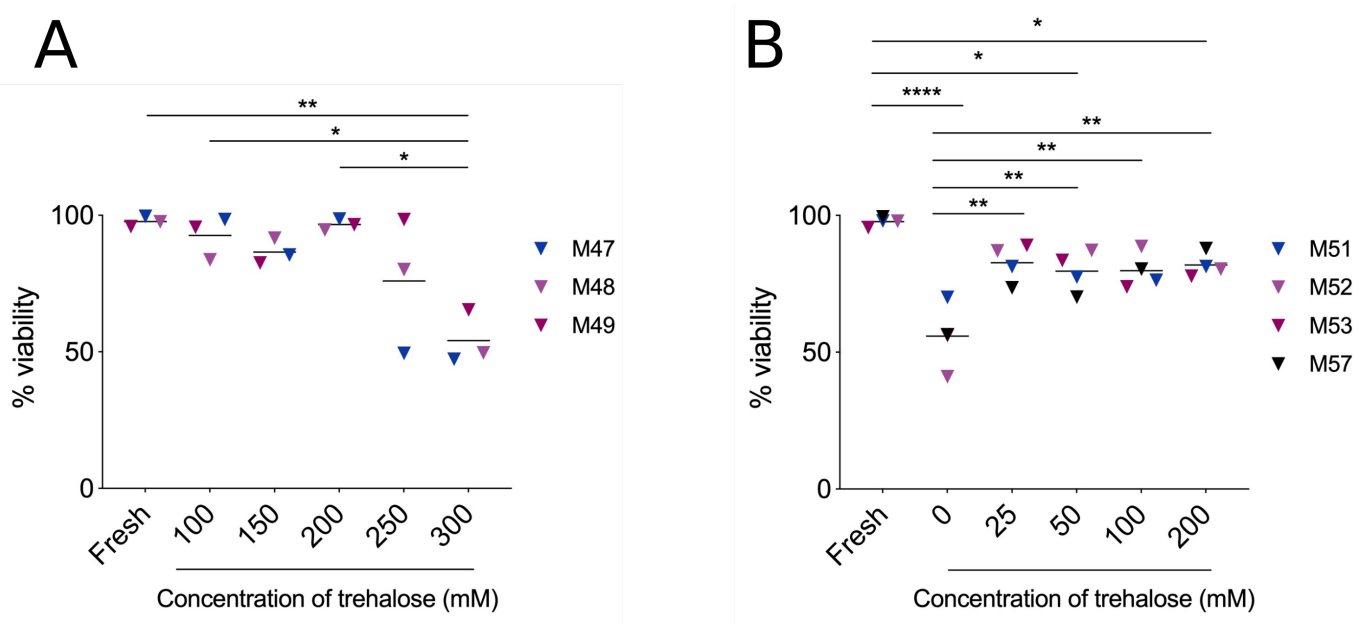

Fig. 5.10 Trehalose concentration in the DT6h protocol. (A) Post-thaw viability of mouse islets cryopreserved using the DT6h protocol with different trehalose concentrations $(100-300 \mathrm{mM})$ measured $24 \mathrm{~h}$ post-thaw $(\mathrm{n}=3$, each value calculated as an average viability of $4-24$ islets). (B) Post-thaw viability of mouse islets cryopreserved using the DT6h protocol with different trehalose concentrations $(25-200 \mathrm{mM})$ measured 24 h post-thaw $(\mathrm{n}=4$, each value calculated as an average viability of $2-35$ islets). Triangles represent average viabilities from individual replicates, triangles of same colour represent results from the same experiment. Horizontal lines represent grand means from the experiments. Repeated measures one-way ANOVA followed with Tukey's HSD post-hoc test was performed, statistically significant difference is marked by asterisks: $* \mathrm{p} \leq 0.05, * * \mathrm{p} \leq 0.01$, $* * * \mathrm{p} \leq 0.001, * * * * \mathrm{p} \leq 0.0001$. 
free systems, which has been demonstrated in this project both for splenocytes (Section 4.5) and pancreatic islets (Section 5.4.1).

Several mechanisms of cryoprotection by trehalose have been proposed in the literature (see Section 1.9.1). It is possible, however, that in the presence of DMSO, some of these protective mechanisms may be redundant (e.g. glass-forming properties) and therefore only the functions which are not performed by DMSO are manifested. These could include the potential antioxidant properties of trehalose or its role in stabilisation of proteins. This may explain why high concentrations of trehalose are not needed in the combined DMSO-trehalose protocol.

\section{Concentration of DMSO}

In the next series of experiments, mouse pancreatic islets were cryopreserved using DT6h cryopreservation protocol with fixed $200 \mathrm{mM}$ trehalose concentration and a range of DMSO concentrations ( $1 \mathrm{M}-2.5 \mathrm{M})$ following protocols shown in Table 5.1. Post-thaw viability was measured immediately and $24 \mathrm{~h}$ after thawing.

Table 5.1 Protocols for DT6h DMSO concentration experiments

\begin{tabular}{llll}
\hline \multicolumn{4}{c}{ Concentrations of DMSO at each step } \\
\hline $\begin{array}{l}\text { Condition } \\
\text { (final concentration) }\end{array}$ & Step 1 & Step 2 & Step 3 \\
\hline 1 M DMSO & $0.33 \mathrm{M}$ & $0.5 \mathrm{M}$ & $1 \mathrm{M}$ \\
1.5 M DMSO & $0.5 \mathrm{M}$ & $0.75 \mathrm{M}$ & $1.5 \mathrm{M}$ \\
2 M DMSO & $0.66 \mathrm{M}$ & $1 \mathrm{M}$ & $2 \mathrm{M}$ \\
2.5 M DMSO & $0.83 \mathrm{M}$ & $1.25 \mathrm{M}$ & $2.5 \mathrm{M}$ \\
\hline
\end{tabular}

Notes: trehalose concentration at all steps was $200 \mathrm{mM}$; RT = room temperature

As can be seen in Figure 5.11A, 1 M DMSO concentration did not provide sufficient protection during cryopreservation of pancreatic islets. However, lowering DMSO concentration from $2 \mathrm{M}$ to $1.5 \mathrm{M}$ did not result in a decrease in efficacy both immediately post-thaw and after one day of culture. Results obtained with the highest 2.5 M DMSO concentration showed large variability.

To check the functionality of islets cryopreserved with the decreased DMSO concentration, static glucose stimulation assay was performed on fresh, 1.5 M DMSO DT6h- and $2 \mathrm{M}$ DMSO DT6h-cryopreserved islets $24 \mathrm{~h}$ after thawing.

The results suggest that responsiveness to different glucose concentrations is preserved in both cryopreserved groups but the 1.5 M DT6h-cryopreserved islets showed insulin secretion 

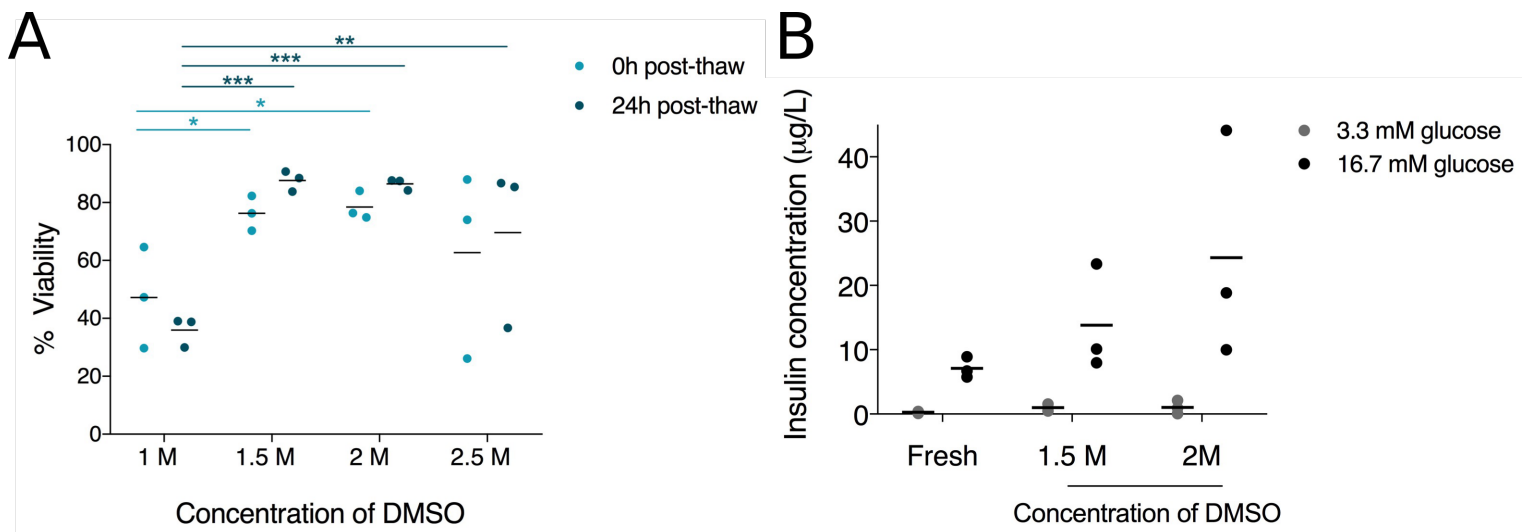

Fig. 5.11 DMSO concentration in the DT6h protocol. (A) Post-thaw viability of mouse islets cryopreserved using the DT6h protocol with different DMSO concentrations $(1-2.5 \mathrm{M})$ measured immediately post-thaw ( $\mathrm{n}=3$, each value is an average viability of $7-24$ islets). (B) Glucosestimulated insulin secretion assay results measured on fresh islets and islets cryopreserved in DT6h protocol (1.5 or $2 \mathrm{M}$ DMSO) measured $24 \mathrm{~h}$ post-thaw. Full circles represent average viabilities/insulin concentrations from individual replicates $(n=3)$, horizontal lines represent grand means from the three experiments. Repeated measures two-way ANOVA followed with Tukey's HSD post-hoc test was performed, significant differences are marked by asterisks: $* \mathrm{p} \leq 0.05, * * \mathrm{p} \leq 0.01 ; * * * \mathrm{p} \leq 0.001$.

profile more similar to the fresh islet group (Figure 5.11B). No significant differences were found between the three groups.

In conclusion, these experiments suggest that lowering DMSO concentration to $1.5 \mathrm{M}$ can be performed without negatively affecting viability and can potentially help to decrease the dysregulation in glucose-stimulated insulin secretion. Decreasing DMSO concentration during cryopreservation has been explored may times in the literature for clinically-relevant cell types but has only been marginally explored for pancreatic islets. In the publication by Lakey et al. [77], a reduction of DMSO concentration from $2.0 \mathrm{M}$ to $1.5 \mathrm{M}$ was explored (without any trehalose addition). The differences between the experiments in this publication and the current study include source of islets (human donors), different glucose concentration in the stimulation assay $(2.8 \mathrm{mM}$ and $20 \mathrm{mM})$ and different measure of recovery (counting of islets as opposed to FDA/PI staining). Nevertheless, the authors came to the same conclusion, that lowering DMSO concentration to $1.5 \mathrm{M}$ could have a beneficial effect.

Outstanding experiments include testing the lower concentrations of both DMSO and trehalose, as well as exploring the functionality of the islets after cryopreservation using this optimised DT6h protocol. Because these DT6h optimisation experiments have not yet been finalised, the original concentrations (2 M DMSO and $200 \mathrm{mM}$ trehalose) were used in all following experiments. 


\subsection{Addition of antioxidants: MitoQ and salidroside}

\subsubsection{Effect of antioxidants during cryopreservation}

A range of cellular stresses are present during freezing and thawing. Cryopreservation methods which combine selected compounds to comprehensively address all these harmful events have the potential to be the most successful. Accumulation of ROS is one of the significant stressors present during cryopreservation, possibly due to the presence of DMSO [195, 196], and can lead to damage of DNA, proteins and lipids, with potentially serious implications for the function of the cells. Many publications have shown that antioxidants can improve the outcome of cryopreservation [156, 132, 197], including in pancreatic islet cryopreservation [154].

Because pancreatic islets are highly sensitive to oxidative stress, use of antioxidants in treatment of diabetic patients has been suggested [198]. Their use could therefore not only provide additional support for survival of cells during cryopreservation but also deliver protective effect to islets during in vitro culture or transplantation.

Salidroside was chosen because it has shown a great promise in red blood cell cryopreservation [156] and evaluation of its efficacy in pancreatic islets would provide a novel protocol. Because ROS are primarily generated in mitochondria, a more targeted approach to elimination of ROS may be beneficial. Therefore, another antioxidant, mitochondria-targeted MitoQ, was chosen as the next candidate.

\section{Salidroside}

Using the two cryopreservation protocols - the standard DMSO protocol and the newly developed DT6h protocol, the effects of salidroside and MitoQ on post-thaw viability of islets were tested. Mouse pancreatic islets were pre-incubated for $6 \mathrm{~h}$ at $37^{\circ} \mathrm{C}$ with culture medium (all DMSO-based samples) or $200 \mathrm{mM}$ trehalose (all DT6h-based samples) and with or without $200 \mu \mathrm{M}$ salidroside or $100 \mathrm{nM}$ MitoQ. The optimal antioxidant concentrations were determined in a series of preliminary toxicity and cryopreservation efficacy experiments using whole and dissociated pancreatic islets (data not shown). The antioxidants were present at this concentration for the whole duration of cryopreservation $(6 \mathrm{~h}$ pre-incubation, freezing, thawing) and during the $24 \mathrm{~h}$ post-thaw culture. Viability of the islets was measured immediately post-thaw and $24 \mathrm{~h}$ post-thaw.

Addition of salidroside led to significant increase of the viability $24 \mathrm{~h}$ after thawing in the original DMSO protocol (mean values of $64.7 \pm 3.9 \%$ with salidroside vs. $39.1 \pm 4.9 \%$ without salidroside, $\mathrm{p}=0.0274$, Figure 5.12A and B). No clear trend was detected after addi- 

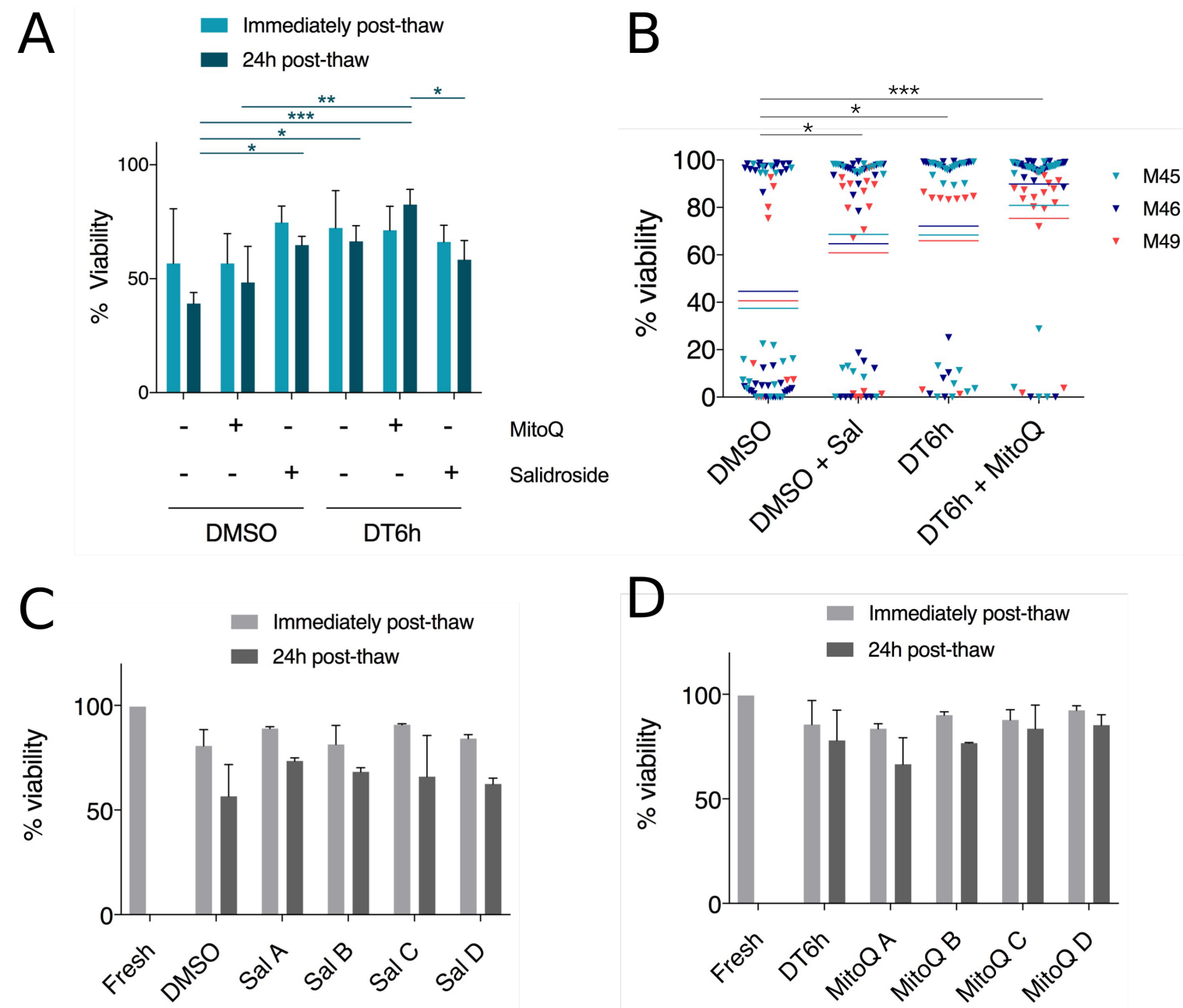

Fig. 5.12 Effect of salidroside and MitoQ antioxidants addition on post-thaw viability of pancreatic islets. (A) Post-thaw viability of mouse islets cryopreserved using DMSO or DT6h protocol with or without $200 \mu \mathrm{M}$ salidroside or $100 \mathrm{nM}$ MitoQ immediately and $24 \mathrm{~h}$ post-thaw $(\mathrm{n}=3$, average viability in each experimental condition calculated from 5-35 islets). (B) Detail of selected conditions from (A): individual islets from all three replicates are shown as full triangles, triangles of same colour represent results from the same experiment. Horizontal lines in the same colour represent means from the three experiments. (C) Post-thaw viability of mouse islets cryopreserved using DMSO with or without $200 \mu \mathrm{M}$ salidroside added at four different stages of cryopreservation, measured immediately and $24 \mathrm{~h}$ post-thaw $(\mathrm{n}=2$, average viability in each experimental condition calculated from $5-38$ islets). (D) Post-thaw viability of mouse islets cryopreserved using DT6h protocol with or without $100 \mathrm{nM}$ MitoQ added at four different stages of cryopreservation, measured immediately and $24 \mathrm{~h}$ post-thaw ( $\mathrm{n}=2$, average viability in each experimental condition calculated from $8-27$ islets). Bars represent mean $\pm \mathrm{SD}$, for data measured immediately post-thaw in (A), repeated measures one-way ANOVA followed with Tukey's HSD post-hoc test was performed, statistically significant differences are marked by asterisks: $* \mathrm{p} \leq 0.05, * * \mathrm{p} \leq 0.01$. DT6h $=\mathrm{DMSO}+$ trehalose $6 \mathrm{~h}$ pre-incubation; Sal $=$ salidroside 
tion of salidroside to the new DT6h protocol $(58.3 \pm 8.4 \%$ with salidroside vs. $66.4 \pm 6.8 \%$ without salidroside, Figure 5.12A).

\section{MitoQ}

MitoQ did not increase viability of DMSO-cryopreserved pancreatic islets $(48.4 \pm 15.8 \%$ with MitoQ vs. $39.1 \pm 4.9 \%$ without MitoQ; Figure 5.12A). However, there was a trend of an improvement on the newly-developed DT6h protocol (82.6 $\pm 6.7 \%$ with MitoQ vs. $66.4 \pm 6.8 \%$ without MitoQ), consistently in all three replicates (Figure 5.12B).

Although no significant differences were found immediately after thawing (other than significantly lower viability of DMSO and DMSO + MitoQ compared to fresh islets), there seems to be a benefit on the survival during the first day after thawing when salidroside was present in the DMSO protocol and when MitoQ was present in the DT6h protocol.

Salidroside did not provide additional protection when added to the DT6h protocol, perhaps because the protective action of trehalose and salidroside partially overlaps. Trehalose has been previously shown to exhibit certain antioxidant effect $[199,197,124]$ and since both of these compounds would act as non-targeted antioxidants, adding both of these at the same time may be redundant. The demonstrated beneficial effect of salidroside complements the findings of Alotaibi et al. [156] for cryopreserved sheep erythrocytes where a significant difference in cryosurvival was found in glycerol-cryopreserved cells but the effect was minimal in cells cryopreserved with trehalose.

To conclude, it was demonstrated that addition of salidroside to the original DMSO protocol and addition of MitoQ to the new DT6h protocol has the potential to further improve cryopreservation. These two protocols were therefore evaluated for their in vitro efficacy in the following Section 5.7.

\subsubsection{Stage of antioxidant addition}

To establish when during the cryopreservation protocol the antioxidants exhibit their protective effect, the antioxidants were added at different stages of cryopreservation as summarised in Table 5.2. Viability of islets from each condition was measured immediately post-thaw and after $24 \mathrm{~h}$ post-thaw culture.

\section{Salidroside}

No obvious trend in viability immediately post-thaw could be observed after adding salidroside at different stages of cryopreservation. After the $24 \mathrm{~h}$ post-thaw culture, the highest viability was measured for the Sal A protocol (73.4 $\pm 1.5 \%$; Figure 5.12C). Late addition 
Table 5.2 Design of antioxidants addition stage experiments

\begin{tabular}{lcccc}
\hline \multirow{2}{*}{ Protocol name } & \multicolumn{4}{c}{ Presence of antioxidant at this step: } \\
\cline { 2 - 5 } & 6 h pre-incubation & Freezing & Thawing & Post-thaw culture \\
\hline Sal/MitoQ A & $\mathrm{X}$ & $\mathrm{X}$ & $\mathrm{X}$ & $\mathrm{X}$ \\
Sal/MitoQ B & & $\mathrm{X}$ & $\mathrm{X}$ & $\mathrm{X}$ \\
Sal/MitoQ C & & & $\mathrm{X}$ & $\mathrm{X}$ \\
Sal/MitoQ D & & & & $\mathrm{X}$ \\
\hline
\end{tabular}

Notes: X marks presence of antioxidant (salidroside or MitoQ) at the particular step of the cryopreservation protocol

of the antioxidant may result in lower viability but the differences could not be statistically tested due to a low number of replicates $(62.4 \pm 2.9 \%$ in Sal D).

\section{MitoQ}

Similar to the salidroside experiments, there were no observable differences between the viabilities in individual conditions immediately post-thaw but a trend could be observed after $24 \mathrm{~h}$ culture. In the case of MitoQ, early addition of the antioxidant (MitoQ A protocol) resulted in the lowest viability of all four tested protocols (66.6 $\pm 12.7 \%$; Figure 5.12D). The highest viability was measured in the MitoQ D protocol, where the antioxidant was added during the post-thaw culture only $(85.3 \pm 5.0 \%)$. Number of replicates in the antioxidant addition stage experiments was too low to perform any statistical analysis.

In the antioxidant addition stage experiments, all the post-thaw viabilities were very high and it is therefore difficult to demonstrate a clear difference between the conditions. However, when looking at the results at $24 \mathrm{~h}$ after thawing, it is apparent that may be advantageous to add salidroside early, during the $6 \mathrm{~h}$ pre-incubation. Another set of experiment would need to be designed to show whether it needs to be present for the whole duration of cryopreservation, thawing and post-thaw culture, or whether it is sufficient to pre-incubate with salidroside and then cryopreserve with DMSO only without the antioxidant. Potential toxicity of salidroside has not been tested yet but if there is no toxic effect present at the salidroside concentration used, there may be no deletrious effect of keeping the antioxidant present. However, simplifying the protocol could be beneficial in a clinical setting.

MitoQ addition to DT6h protocol demonstrated different trend to the salidroside experiment: it was sufficient to add MitoQ for the post-thaw culture to achieve the same efficacy. This may seem as a conflicting result to the one shown if Figure 5.12A, where MitoQ was present for the whole duration of cryopreservation and yet exhibited higher post-thaw via- 
bility than DT6h alone. It must, however, be pointed out that the number of replicates was low in these experiments and that the viability in this last set of experiments was unusually high in all conditions and therefore it may be difficult to demonstrate an improvement to the DT6h viability.

Although these results are still inconclusive, this data suggests that salidroside should be added at an early time point, ideally during the $6 \mathrm{~h}$ pre-incubation period, to be efficient, while it may be sufficient to add the MitoQ for the thawing or post-thaw culture. Additional experiments must be performed to confirm these findings.

\subsection{Function of islets cryopreserved by new protocols}

\subsubsection{In vitro efficacy}

Most previous experiments used viability as the measure of the cryopreservation outcome. This was ideal for screening and development of new successful cryopreservation protocols but results provided by the viability assay are too crude and must be validated using a range of assays looking at health and function of the cryopreserved islets before proceeding to clinical tests. Some of the newly developed cryopreservation methods were therefore compared to fresh islets and DMSO-cryopreserved islets in the first instance in vitro using mitochondrial DNA (mtDNA) oxidation assay, ATP/ADP ratio assay, glucose-stimulated insulin secretion (GSIS) assay and cyclic AMP (cAMP) release assay.

For all of the assays, the islets were cryopreserved using previously described protocols. After thawing, the islets were cultured for $24 \mathrm{~h}$ and then used to perform one or more different functional assays. ATP/ADP ratio assays and mtDNA oxidation assays were performed in collaboration with Anja Gruszczyk and cAMP release assay with Dr. Kerry Barkan. Optimisation of the in vitro functional assays and their analysis was performed and results can be found in Appendix D.

\section{Mitochondrial health and metabolic status}

As expected, the lowest levels of mtDNA oxidation (highest amplification number) was measured for fresh pancreatic islets. DMSO and DT6h had comparable levels of oxidative damage in their mtDNA, both being significantly worse than fresh islets (Figure 5.13A). There was a trend of smaller damage of the mtDNA upon addition of the antioxidants but the differences were not statistically significant $(\mathrm{p}=0.9050$ for DMSO vs. DMSO + Sal; $\mathrm{p}=0.4845$ for DT6h vs. DT6h + MitoQ). 
A
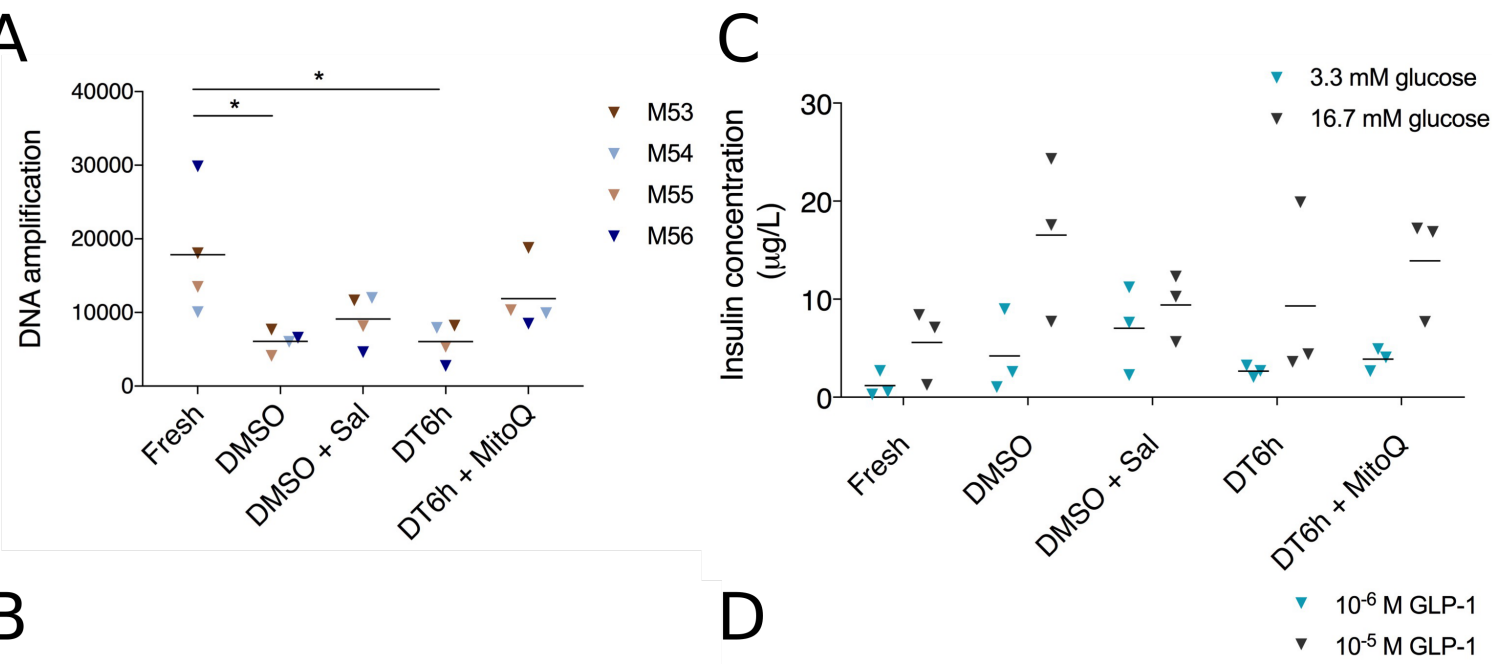

B
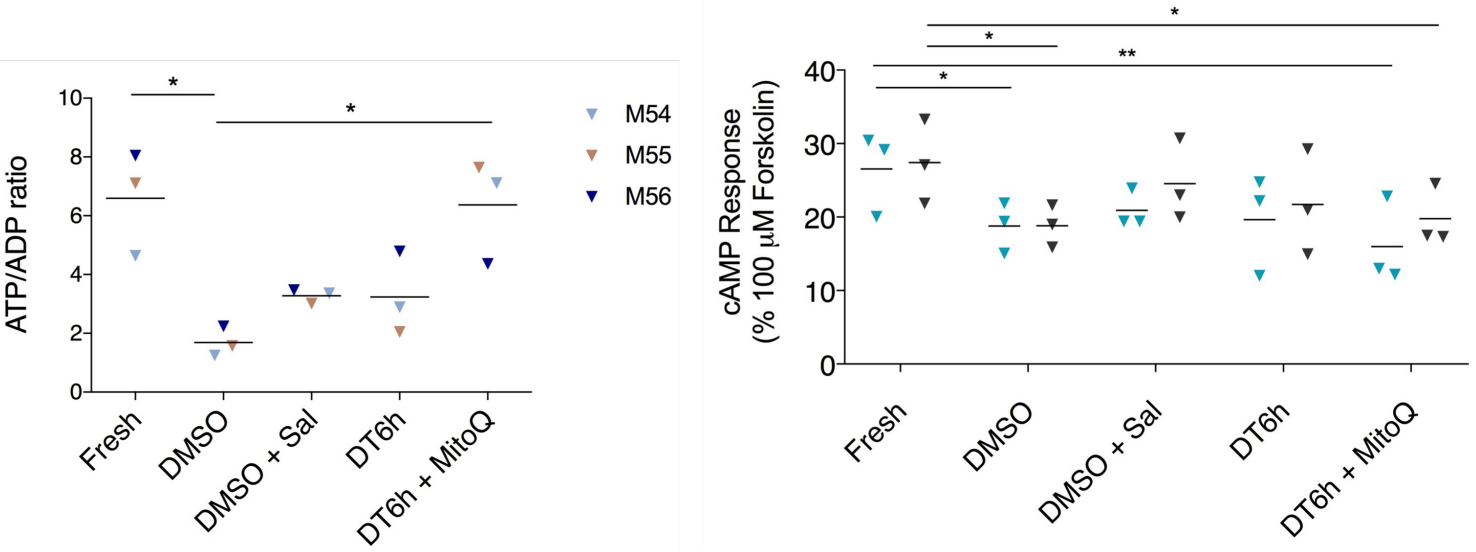

Fig. 5.13 In vitro function of pancreatic islets cryopreserved by the optimised protocols. Cryopreservation conditions tested include DMSO, DMSO + 200 $\mu$ M salidroside, DT6h, DT6h + $100 \mathrm{nM}$ MitoQ. All functional assays were performed at the end of $24 \mathrm{~h}$ post-thaw culture. (A) Mitochondrial DNA oxidation levels of fresh and cryopreserved islets. Higher amplification numbers indicate lower oxidation of mitochondrial DNA $(n=4)$. (B) ATP/ADP ratios in fresh and cryopreserved islets. Higher ratios indicate better health of the cells $(\mathrm{n}=3)$. (C) Insulin secretion of fresh and cryopreserved islets stimulated with $3.3 \mathrm{mM}$ and $16.7 \mathrm{mM}$ glucose $(\mathrm{n}=3)$. (D) Cyclic APM release by fresh and cryopreserved islets after stimulation with two concentrations of GLP-1 ( $n=3)$. In (A) and (B), results from the same experimental sample are depicted as triangles of the same colour. Horizontal lines in the same colour represent grand means of the three experiments. In (C) and (D), triangles represent average values from individual replicates, horizontal lines represent grand means of the three experiments. Repeated measures one-way or two-way ANOVA followed with Tukey's HSD post-hoc test was performed as appropriate, statistically significant results are marked by asterisks: $* \mathrm{p} \leq 0.05, * * \mathrm{p} \leq 0.01$. DT6h $=$ DMSO + trehalose $6 \mathrm{~h}$ pre-incubation; Sal $=$ salidroside 
ATP/ADP assay showed similar results, with fresh islets having the highest ATP/ADP ratios and DMSO-cryopreserved islets having the lowest (Figure 5.13B). Addition of salidroside (DMSO + Sal) or trehalose (DT6h) led to a slight, statistically non-significant increase. This time, DT6h + MitoQ had very similar ATP/ADP ratios to fresh islets (6.4 $\pm 1.8 \mathrm{vs}$. $6.6 \pm 1.8, \mathrm{p}=0.9996$, respectively), and both DT6h + MitoQ and fresh islets had significantly higher ratios than DMSO-cryopreserved islets $(1.7 \pm 0.5, p=0.0208$ and $p=0.0161$, respectively).

Consistently high mtDNA amplification numbers and ATP/ADP ratios of fresh islets confirmed good health of the islets before cryopreservation. These two assays also showed the amount of oxidative and other damage done to the mitochondria during cryopreservation and that some of this damage may be reduced using appropriate additives during cryopreservation. Low mtDNA oxidation levels and high ATP/ADP ratios of islets cryopreserved in DT6h + MitoQ are not surprising either. Both of these measures are describing the health and function of mitochondria - the target of MitoQ. Salidroside was able to reduce some of the harmful effects of cryopreservation on mitochondria but since its action is non-targeted, the overall effect on cell survival may not be visible using these two assays.

\section{Responsiveness to glucose}

In the GSIS assay, the ratio of insulin concentration in high and basal glucose concentrations (stimulation index) was 4.72 for fresh islets. The lowest stimulation index was measured for DMSO + salidroside (1.34) but even in this condition, the islets retained some responsiveness to changing glucose levels. The stimulation index for the remaining three conditions were comparable (3.94 for DMSO, 3.50 for DT6h and 3.59 for DT6h + MitoQ, data not shown). The graph in Figure 5.13C shows the actual insulin concentrations (not normalised to DNA) measured at basal and high glucose concentrations to illustrate the variability of the results and similarity to the stimulation profile of fresh islets.

Interpretation of the result of the GSIS assay is complex. Islets in all the cryopreservation samples remained responsive to glucose but comparison of the individual protocols is difficult. While high secretion of insulin is in many publications regarded as a sign of good health of islets, it may be concerning that all the cryopreserved islets secrete higher levels of insulin than fresh islets even at the basal level of glucose $(3.3 \mathrm{mM})$. This could indicate either a passive release of insulin from dying cells or dysregulation of insulin release pathways. If such dysregulated islets were to be used for transplantation, it could potentially lead to suboptimal clinical efficacy. The most optimal cryopreservation should therefore have similar levels of insulin secretion at basal level. From this point of view, DT6h and DT6h + MitoQ appear to have the lowest and least variable insulin production when incubated in $3.3 \mathrm{mM}$ 
glucose solution and can therefore be regarded as the most successful cryopreservation conditions.

\section{Responsiveness to incretin hormones}

Cyclic AMP release upon stimulation with incretin hormone GLP-1 produced the highest response in fresh pancreatic islets $(26.5 \pm 5.66$ and $27.4 \pm 5.7 \%$ of forskolin response for $10^{-6} \mathrm{M}$ and $10^{-5} \mathrm{M}$ GLP-1 concentrations, respectively; Figure 5.13D). There was cAMP production in all cryopreservation conditions, suggesting that the cellular machinery remains intact, but no obvious differences were observed between the different cryopreservation protocols.

Not a lot of difference could be seen between stimulations with two different concentrations of GLP-1. One of the possible explanations may be that both of the tested GLP-1 concentrations are very high and even with the lower concentration, the cells are already stimulated to their near-maximal level. Further experiments are, therefore, required with lower concentrations of GLP-1.

To summarise, fresh islets provided a good control in all the four assays. DMSO led to the highest levels of mtDNA oxidation, lowest ATP/ADP ratios, dysregulated release of insulin and low responsiveness to different GLP-1 concentrations, and although these trends are not statistically significant, it can be ranked as the worst of all tested cryopreservation conditions. The decision about the best condition is more complicated. In terms of mitochondrial health, characterised by the mtDNA oxidation and ATP/ADP ratio, DT6h + MitoQ performed the best, likely owing to MitoQ's ability to target ROS specifically in mitochondria. In the stimulation assays, however, DT6h seemed to have insulin and cAMP secretion profile more similar to the one of fresh islets than DT6h + MitoQ. Further experiments are required to clarify these trends.

\subsubsection{In vivo efficacy}

As a final confirmation of the efficacy of the new cryopreservation protocols, in vivo model of STZ-induced diabetes in NSG mice was developed and initial experiments performed using this model. Three preliminary experiments to determine the correct dose of STZ and transplanted pancreatic islets were performed and their results are summarised in the Appendix E.

NSG mice were injected i.p. with $40 \mathrm{mg} / \mathrm{kg} \mathrm{STZ} \mathrm{for} \mathrm{five} \mathrm{consecutive} \mathrm{days} \mathrm{and} \mathrm{trans-}$ planted with 400 fresh, DMSO-cryopreserved or DT6h-cryopreserved mouse pancreatic islets (from C57BL/6JAX mice) under the kidney capsule 16 days after the first STZ injection 

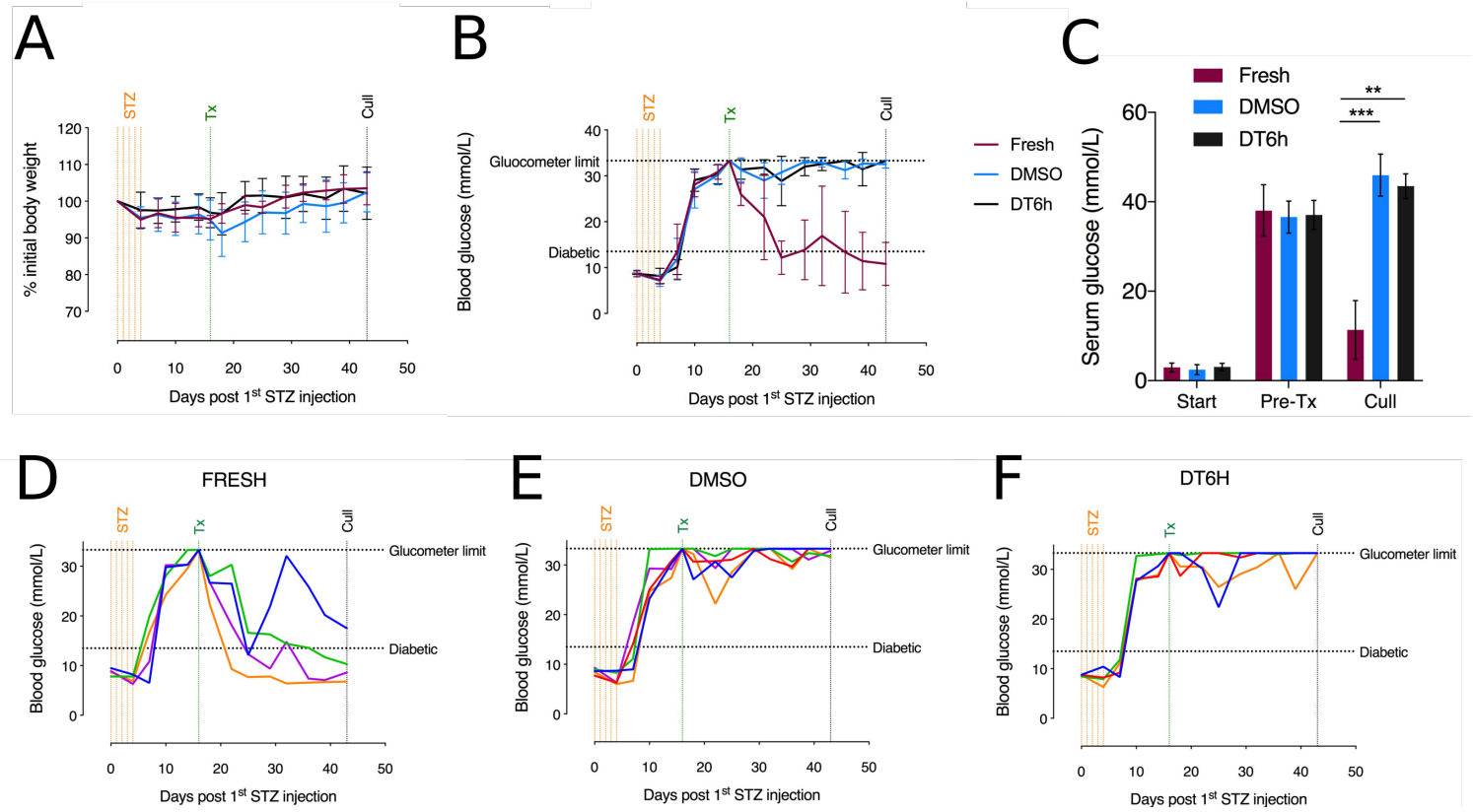

Fig. 5.14 In vivo function of pancreatic islets cryopreserved with DMSO and DT6h protocols. Days of STZ injections (STZ), islet transplantations under kidney capsule (Tx) and culling of the animals (Cull) are indicated in the graphs by vertical lines. (A) Weight losses in the three groups of STZ-treated diabetic animals over the duration of the experiment ( $n=4-5$ animals per group). (B) Blood glucose levels measured by portable glucose meter in the three groups of STZ-treated diabetic animals over the duration of the experiment ( $\mathrm{n}=4-5$ animals per group). Data points represent mean $\pm \mathrm{SD}$. (C) Blood serum glucose levels in the three groups of STZ-treated diabetic animals at the beginning of the experiment (Start), prior to transplantation (Pre-Tx) and the end of the experiment (Cull) measured by biochemical test in CBAL laboratory ( $\mathrm{n}=4-5$ animals per group). Bars represent mean \pm SD. Two-way mixed-effects model ANOVA followed by Tukey's HSD post-hoc test was performed, statistically significant differences are marked by asterisks: $* * \mathrm{p} \leq 0.01, * * * \mathrm{p} \leq 0.001$. (D) Blood glucose levels measured by portable glucose meter in the individual mice of the FRESH group over the duration of the experiment $(n=4)$. (E) Blood glucose levels measured by portable glucose meter in the individual mice of the DMSO group over the duration of the experiment $(n=5)$. (F) Blood glucose levels measured by portable glucose meter in the individual mice of the DT6h group over the duration of the experiment $(n=4)$. All experimental animals shown were tested concurrently. $\mathrm{DT} 6 \mathrm{~h}=\mathrm{DMSO}+$ trehalose $6 \mathrm{~h}$ pre-incubation; Pre-Tx $=$ before transplantation; STZ = streptozotocin; $\mathrm{Tx}=$ transplantation. 
(5 mice per group). The animals were randomly allocated to the groups. Any animals receiving lower dose of pancreatic islets (due to technical error or shortage of islets) were excluded from the analysis at the end. Body weights and blood glucose were monitored twice a week and blood serum was collected at the start and end of the experiment, as well as on the day of the transplantation for biochemical measurement of glucose in the CBAL laboratory. Animals were culled 4 weeks after transplantation.

After the initial drop in weight following the daily STZ injections, the body weights stabilised in all animals (Figure 5.14A). After transplantation, there was a slight drop in the weight of the DMSO group but eventually, the weights started to rise in all three groups. Towards the end of the experiment, the weight gain stopped in the DMSO and DT6h groups.

Blood glucose was well matched in all three groups before the transplantation and reached the portable glucometer limit of $33.3 \mathrm{mmol} / \mathrm{L}$ on the day of the transplantation in all animals (Figure 5.14B). Transplantation of 400 fresh islets led to a rapid decrease in blood glucose levels which led to the average blood glucose in this group being under the diabetic threshold of $13.5 \mathrm{mmol} / \mathrm{L}$ in 9 days after transplantation. When looking at the individual animals in this group, in 3 out of 4 the blood glucose kept decreasing until the end of the experiment and in one animal the glucose started rising again after Day 9 post-transplantation (Figure 5.14D).

In the two cryopreserved groups (DMSO and DT6h), transplantation of 400 islets did not lead to a decrease in blood glucose levels during 4 weeks post-transplantation (Figure 5.14B). This was true not only on average in the two groups but also for all the individual animals, although temporary decrease in blood glucose was seen in several animals in both groups (Figures 5.14E and F). Towards the endpoint, many animals from the DMSO and DT6h groups had blood glucose higher than the glucose meter limit. However, blood serum was collected at the start of the experiment, before transplantation and before culling to enable more precise measurement of serum glucose in CBAL biochemical laboratory.

These results showed that animals in all three groups had similar glucose levels at the beginning of the experiment and before transplantation. At the end of the experiment, there was significant difference between FRESH and DMSO $(p=0.0007)$ and FRESH and DT6h $(\mathrm{p}=0.0025)$. There was no significant difference between DMSO and DT6h group (Figure 5.14C).

No significant weight loss was observed over the course of the experiment in any of the mice, likely because of the reduced dose of STZ (see Appendix E) and feeding by mashed food for the whole duration of the experiment. The small decline in body weights near the end of the experiment in the DMSO and DT6h groups was likely caused by long-term exposure of these mice to very high blood glucose levels ( $>4$ weeks of $\sim 30 \mathrm{mmol} / \mathrm{L}$ glucose). No other side effect of the prolonged period of diabetes were observed in these mice. 
Some variability was observed in the consecutive measurements of the individual mice. Any obvious technical errors in measurements by the glucose meter were excluded but some variability still remains. One of the reasons could be that the animals were not fasted prior to the glucose measurements and even though the measurements were performed at the same time of the day, it could therefore not be monitored whether the animals have eaten or not. Nevertheless, the blood glucose level development was still obvious despite this relatively small level of variability.

The DT6h protocol showed improvement in the in vitro tests of viability and function over the standard DMSO protocol. However, this difference was not demonstrated by this initial in vivo experiment. Different design of the experiment may perhaps be needed which will test different dose of the cryopreserved islets and determine the minimal effective dose for each of the cryopreserved islets. Sectioning and staining of paraffin-embedded transplanted kidneys will also reveal whether any islets in the DMSO and DT6h groups under the kidney capsule survived.

To conclude, a functional model of STZ-induced diabetes in NSG mice was generated and used to test the ability of fresh and cryopreserved islets to reverse diabetes. While fresh islets rapidly decreased blood glucose to non-diabetic levels after transplantation, both DMSO- and DT6h-cryopreserved islets did not achieve this at the dose used (400 islets per mouse) in this initial experiment. Further experiments are required to establish the minimal dose of cryopreserved islets for reversal of diabetes in mice.

\subsection{Discussion}

\subsubsection{Cryopreservation of islets with trehalose}

In this Chapter, the ultimate aim was to develop an improved cryopreservation protocol for mouse pancreatic islets. The current standard protocol uses DMSO, with documented toxicity for islets, can only preserve about $50 \%$ of the islet mass, as it was demonstrated for both mouse and human pancreatic islets in this project.

The above experiments showed that the ability of trehalose to fully replace the cryoprotective effect of DMSO is limited. Even when sufficient time for diffusion of trehalose to all cells was allowed, trehalose still performed significantly worse than DMSO. In line with the results for splenocytes in Chapter 4, addition of permeabilising polymers PP-50 and PP-75 did not lead to improvement of post-thaw viability in preliminary experiments. It has not been determined whether this was because of the non-functionality of the polymers or limited cryoprotective properties of trehalose even after intracellular loading. 
Addition of trehalose to penetrating cryoprotectants has been shown to improve cryopreservation of several cell types, including pancreatic islets, but the addition is typically performed in a single step without incubation. In the publication by Beattie et al. [83], fetal islet-like cell clusters and adult human pancreatic islets were cryopreserved in a mixture of $300 \mathrm{mM}$ trehalose and $2 \mathrm{M}$ DMSO. Although the published protocol is not very detailed, the trehalose was presumably added at the same time as DMSO, likely 45 min prior to freezing. From the data produced in this project, it is clear that using such protocol, the full potential of trehalose in islet cryopreservation would not be realised. The data available in this Chapter systematically evaluated parameters involved in cryopreservation, allowing to maximise the protective effect of trehalose.

Firstly, 45 min of incubation is not a sufficient time for equilibration of trehalose in the whole volume of pancreatic islets. In this project, the ideal duration of pre-incubation with trehalose for cryosurvival of islets was experimentally determined here to be $6 \mathrm{~h}$ at $37{ }^{\circ} \mathrm{C}$. Interestingly, the incubation time in the diffusion experiments from Chapter 3 which led to equilibration of islets with nuclear dye was also $6 \mathrm{~h}$. The assumption that the diffusion rates measured in Chapter 3 could serve as a prediction for behaviour of other small molecules in aqueous solutions therefore seems to be accurate.

Secondly, $300 \mathrm{mM}$ trehalose used in the publication in combination with $2 \mathrm{M}$ DMSO may have been a source of increased osmotic stress, as shown in Section 5.5.2. Although the experiments to determine ideal concentration of both cryoprotectants have not been finalised yet, it is clear that some of this stress can be eliminated by decreasing the concentration of one or both of them. Regarding the ideal concentration of trehalose, an interesting observation was made: the ideal concentration of trehalose was $200 \mathrm{mM}$ when used as the sole cryoprotectant but could be decreased down to $25 \mathrm{mM}$ when used in combination with DMSO.

It is currently not clear for the newly developed protocol whether trehalose provides protection intra- or extracellularly because intracellular trehalose uptake in pancreatic islets was not measured as part of this project. It is expected that there is a very small amount of passive diffusion of trehalose through the membrane but this would not increase the intracellular trehalose concentration significantly. Beattie et al. [83] raised another very interesting point about entry of trehalose to the intracellular space during cooling thanks to lipid-phase transition of the membrane resulting in increased permeability. If this is correct and lipid-phase transition indeed allows trehalose to enter cells, it certainly would be advantageous and no other additives or interventions which could potentially complicate translation of this method into clinical practice would be required. 


\subsubsection{New insight into cell cluster survival}

The present studies offer potentially important insights into the long-term survival of pancreatic islets. The data suggest that a critical mass of live cells within islets is needed to support the survival of the whole cell cluster. The nature of this intra-cluster communication has not been investigated yet but it is likely that apoptotic pathway is being activated due to the loss of attachment to other living cells or extracellular matrix. Similar process has been described for many adherent cell types which need attachment to extracellular matrix to survive, otherwise they undergo a type of apoptosis which is referred to as 'anoikis' [200]. Recently, the importance of cell-cell contact mediated by cadherins, selectines and other adhesion molecules for survival of certain cell types is being discovered. Metastatic tumor cells are able to avoid anoikis by activation of certain signalling pathways [201]. It may be possible, for example, to treat islets with a therapeutic agent after thawing which would transiently activate or block the affected pathway(s), preventing anoikis in the crucial period after cryopreservation.

Analysis of transcriptome could reveal which receptors or pathways are involved in survival of islets or trigger of apoptosis. Time-lapse imaging of islets after thawing could then bring some insight into the re-formation of the surviving cells into a smaller functioning islet.

\subsubsection{Cryopreservation with antioxidants}

There has been an increased interest in addition of antioxidants into cryopreservation media for different cell types in the recent years. However, action of antioxidants in pancreatic islet cryopreservation has not been extensively studied. Kanitkar et al. [154] used curcumin as an additive to the standard DMSO protocol and showed that it improved post-thaw recovery and decreased whether it was added during cryopreservation or only in the post-thaw culture. This project evaluated the efficacy of another natural non-targeted antioxidant salidroside in pancreatic islet cryopreservation for the first time and showed similar results - although from the preliminary data it is apparent that early addition of salidroside provides more benefit than adding it in post-thaw culture only.

Trehalose has also been shown to possess antioxidant properties [199, 197, 124]. Addition of antioxidants with similar action to cryopreservation media containing trehalose could therefore be redundant. In this Chapter, salidroside was shown to improve survival of islets in the DMSO-based protocol but failed to improve the DT6h protocol, possibly because of this reason. MitoQ, on the other hand, exhibits its antioxidant properties mainly in mitochondria, 
where it accumulates. Combination of several antioxidants with different actions (targeted vs. non-targeted etc.) could be one of the approaches to minimise oxidative damage.

Similarly to antioxidants, other additives could supplement the newly developed DMSO + trehalose protocol. As long as they lack toxicity, they can be added during the $6 \mathrm{~h}$ preincubation step, allowing them to diffuse to all cells of the islet. These molecules could include nucleation inhibitors or antifreeze proteins to reduce the amount of damage during cryopreservation, or molecules which specifically target some of the sites of damage (such as anti-apoptotic agents).

The outstanding experiments include additional validation of the cryopreserved islet quality and safety of the developed protocols. The assays used in this Chapter to evaluate islet function looked at production of insulin and cAMP in islets which very specifically indicate the function of $\beta$-cells. However, the function of the other cell types present in islets is critical if they are to correctly regulate blood glucose levels after transplantation. More comprehensive evaluation of hormone production could be evaluated using peptidomic analysis. Furthermore, bulk and single-cell RNA sequencing could reveal any important changes happening to the cells during cryopreservation. In addition, because the development of the novel protocols was performed using mouse islets, it must also be demonstrated that the findings can be replicated for human islets too.

\subsection{Conclusion}

This Chapter aimed to develop a novel method of pancreatic islet cryopreservation which could ultimately enable cryopreserved islets to be used for clinical transplantation in the treatment of diabetes. First, several parameters of the DMSO protocol, which is the current standard in the islet cryopreservation research, were investigated and optimised. The development of a new protocol was then approached using two directions: firstly, by complete substitution of the toxic DMSO by trehalose and secondly, by improving the current DMSO protocol using additives.

While the approach using trehalose on its own did not generate a successful protocol, addition of trehalose to the DMSO protocol did improve cryopreservation outcomes, once the correct incubation times were used $(6 \mathrm{~h}$ pre-incubation with trehalose followed by step-wise addition of DMSO in the last $45 \mathrm{~min}$ ). The health and function of islets cryopreserved using this successful newly developed protocol were tested and showed that combination of trehalose and DMSO is superior to DMSO alone not only in post-thaw viability but also other markers of islet function. Superior function in vivo was not yet demonstrated for this 
new protocol in the preliminary experiments on mouse model of STZ-induced diabetes but further experiments will be performed to evaluate this.

This newly generated and comprehensively evaluated DMSO + trehalose protocol was then used as a stepping stone for testing additional additives in order to address the multitude of challenges present in cryopreservation. It was shown that cryopreservation can be further improved by addition of antioxidants, specifically MitoQ and salidroside. The possibility of decreasing the concentration of DMSO without decrease in post-thaw recovery was also outlined which would further improve the safety of the new method in the clinical scenario. 


\section{Chapter 6}

\section{General discussion}

\subsection{Introduction}

This chapter provides a discussion of the findings, their limitations and possible future directions, as well as their implications for the fields of islet cryobiology and clinical islets transplantation.

In Section 6.2, the findings of this project are reviewed with reference to the aims and objectives outlined in Chapter 1.12. The impact of the most important findings is then explored in the context of existing literature in Section 6.3.

Limitations of this project are then outlined and discussed in Section 6.4, followed by suggestions for future work and for translation of the novel cryopreservation protocol into clinical practice in Section 6.5.

\subsection{Research aims and major findings}

The ultimate aim of this project was to develop a method for cryopreservation of pancreatic islets which would provide sufficient viable islet mass to ultimately enable clinical transplantation of cryopreserved pancreatic islets. A novel protocol was designed which uses $6 \mathrm{~h}$ incubation with trehalose followed by step-wise addition of DMSO to achieve improved cryosurvival and in vitro function compared to the standard DMSO protocol. Furthermore, this protocol was further improved by addition of antioxidants MitoQ and salidroside to enhance cryosurvival.

Due to the complexity of the structure of pancreatic islets, a comprehensive evaluation of trehalose and permeabilising polymers PP-50 and PP-75 for mediating the intracellular uptake of trehalose was first performed using human mononuclear cells as a simplified model. 
The potential of the use of these polymers in improving cryopreservation with trehalose was not confirmed in human mononuclear cells and this approach was therefore not pursued further in islet experiments.

A critical step for the development of an improved cryopreservation protocol was the evaluation and improvement of current viability assessment protocols, which was performed for both mononuclear cells and pancreatic islets. Insights into the diffusion kinetics of small molecules determined the ideal incubation time to be $6 \mathrm{~h}$ at $37^{\circ} \mathrm{C}$ and enabled not only generation of an improved staining and imaging protocol for pancreatic islets but also helped with the design of the a novel islet cryopreservation protocol.

\subsection{Impact of the findings}

\subsubsection{Standards of viability assessment in cryobiology}

In Chapter 3 of this project, I identified a need for cryobiology research to adopt common protocols for assessment of cryosurvival and the specific requirements for such an assessment method. Although this concept is not new, this is the first time, to my knowledge, that a comprehensive review of existing literature and original investigations has resulted in a proposal for a standardised method for assessment of cryosurvival. I successfully applied this approach to develop a method for cryosurvival assessment of mononuclear cells which meets all these cryobiology-specific needs.

By dissemination of the findings and proposal from this project, it is hoped that these standards will be adopted by researchers in the cryobiology field, improving the ability to compare findings of studies by different investigators, enhancing data transparency and an unbiased approach.

\subsubsection{Limitations in pancreatic islet viability assessment}

It is well known that the results of pancreatic islet viability assessment do not readily correlate with transplantation outcomes [94]. A potential factor is that viability assessment is performed shortly after the period of ischemia during pancreas storage and a physiologically stressful islet isolation process. However, this project identified additional important factors which contribute to the inaccurate results obtained from viability assessment assays.

A range of imaging modalities were used in this study to assess the viability of the core and periphery of pancreatic islets. Importantly, these studies revealed the inadequacy of current imaging techniques in assessment of viability of the core of islets. While it is previously acknowledged that using imaging to assess islet viability is challenging [95], the 
different methods have not previously been systematically and comprehensively assessed. In this study, four different imaging technologies (wide-field, confocal, multi-photon and light sheet microscopy) for assessment of pancreatic islets were compared. Importantly, I demonstrated that the clinically-used islet viability assay based on FDA/PI dyes does not lead to staining of the whole islet volume but rather only the peripheral cell layers, frequently leading to incorrect estimation of islet viability.

Although other assays, especially those examining physiological function of islets, could be regarded as more insightful, I believe that simple viability staining has an important place in cryobiological assessment for its ability to provide rapid results during screening of cryopreservation conditions. The data from this project generate important insights into the underlying problems of FDA/PI staining and imaging. Addressing these shortfalls can help develop more accurate methods not only for academic studies but also for clinical use, which specially require rapid and reproducible methods. Furthermore, considerations for development of an automated image analysis protocol were discussed in this thesis. Such an approach, once fully developed and validated, could be shared with clinical laboratories for immediate use in clinical islet assessment of fresh (and cryopreserved) islets.

\subsubsection{Diffusion of small molecules into the core of pancreatic islets}

Determining the minimal incubation time $\left(6 \mathrm{~h}\right.$ at $\left.37^{\circ} \mathrm{C}\right)$ for small molecules in aqueous solutions to diffuse throughout pancreatic islets is a key finding of this project. This finding has important implications not only for the design of the new islet staining methods, but also for the development of novel cryopreservation protocols. Unlike for DMSO [72], similar empirical studies have not previously been performed for non-penetrating cryoprotectants.

The findings of these diffusion studies have applications to not only islet cryopreservation, but also for loading of other therapeutic agents into islets. In principal, a range of therapeutics agents (antioxidants, anti-apoptotic agents or other targeted therapeutics) may be used to decrease islet cell death rate in culture or to pre-condition the islets for transplantation. Knowledge of the diffusion kinetics can help to design suitable incubation times and conditions to successfully load islets with therapeutic compounds.

The method presented here for studying diffusion of $\mathrm{H} 33342$ into pancreatic islets was inspired by a study done by Durand et al. [202] where gradient of staining from the periphery to the core of tumor spheroids was used to select cells from different locations using a fluorescent cell sorter. However, H33342 staining has not been previously used to study diffusion kinetics. The method developed in this project can be applied for other multicellular aggregates or even whole organs, through perfusion with a solution of H33342 for defined 
periods followed by fixation and sectioning to reveal penetration of the dye in relation to the vasculature.

\subsubsection{Trehalose potential for cryopreservation of mononuclear cells}

The study presented here provided the most comprehensive optimisation of trehalose cryopreservation reported to date using highly accurate methods to ensure unbiased reporting of post-thaw cell viability. DMSO cryopreserved approximately five fold more mononuclear cells than the optimised protocol using $400 \mathrm{mM}$ trehalose. The findings of this study, therefore, suggest that trehalose alone cannot be used to substitute the cryoprotectant DMSO.

Similar studies which used trehalose as a sole cryoprotectant without intracellular trehalose delivery are limited. Scheinkonig et al. [130] reported comparable viability of mononuclear cells cryopreserved in $500 \mathrm{mM}$ trehalose to DMSO, while $1 \mathrm{M}$ or $250 \mathrm{mM}$ trehalose preserved significantly smaller proportion of viable cells. The colony-forming ability of cells cryopreserved in $500 \mathrm{mM}$ trehalose was improved compared to DMSO. Martinetti et al. [203] reported improved post-thaw viability of $\mathrm{CD} 34^{+}$cells using $1 \mathrm{M}$ trehalose compared to $10 \%$ DMSO.

The difference in the findings of these studies and results reported here may stem from the methods of viability assessment used. Both studies assessed viability using trypan blue staining. However, as demonstrated in Chapter 3, this method of viability assessment, without reporting of cell number, can lead to overestimation of cryosurvival. In addition, the study of Martinetti et al. studied cryosurvival of $\mathrm{CD}^{+} 4^{+}$as opposed to mononuclear cells. Although it has been reported and discussed in Appendix B that the freezing process is less damaging to $\mathrm{CD} 34^{+}$cells than other mononuclear cell types, the results of Martinetti et al. are still surprising since such success of cryopreservation with trehalose as the sole cryoprotectant has not been reported elsewhere for $\mathrm{CD} 34^{+}$cells or any other cell type.

This study did not explore the addition of trehalose to the standard DMSO protocol for the cryopreservation of mononuclear cells. However, this topic has been explored elsewhere [131, 132, 204, 186]. These publications consistently reported improvement of colony forming ability of cell cryopreserved in media supplemented with trehalose compared to DMSO alone. In summary, trehalose may have limited potential in protocols where it is used as the only cryoprotectant but, if used as an additive to the conventional DMSO medium, it can potentially improve the recovery of haematopoietic cells. Efforts should therefore be invested in optimisation of these combined protocols and for the development of more efficient DMSO removal methods. 


\subsubsection{Safety and efficacy of permeabilising polymers in primary cells}

The use of permeabilising biopolymers PP-50 and PP-75 to enhance intracellular delivery of trehalose did not improve cryopreservation of splenocytes or pancreatic islets with trehalose. While efficacy of these polymers has previously been demonstrated for red blood cells [141], their use in nucleated cells has so far been evaluated only in two publications which utilised the SAOS-2 cell line $[142,146]$. This project, however, is the first report on the efficacy of these polymers for the cryopreservation of primary nucleated cells.

Sharp et al. reported an improvement in metabolic activity in trehalose-cryopreserved SAOS-2 cells when PP-50 was added at the concentration of $25 \mu \mathrm{g} / \mathrm{mL}$ but not higher. Additionally, a decrease in metabolic activity for $1000 \mu \mathrm{g} / \mathrm{mL}$ concentration was shown in a toxicity test [142]. Mercado et al. provided additional insights into the cellular localisation of PP-50 and the loaded trehalose. In this report, $25 \mu \mathrm{g} / \mathrm{mL}$ PP-50 was shown to increase post-thaw survival of SAOS-2 cells. The cells were incubated at $\mathrm{pH} 7.05$ with the polymer for $2 \mathrm{~h}$ and $4 \mathrm{~h}$, respectively, in the two studies. However, as shown in this project, incubation of splenocytes at $\mathrm{pH} 7.05$ has a detrimental effect on their viability. Long incubations, which may be necessary for the function of these polymers, could therefore not be utilised for these primary cells.

This project also identified additional safety concerns related to the use of these polymers. While concentrations of polymers which do not result in a decrease of cell viability were determined both here and in previous publications, long-term effects on cells and other safety concerns, such as biodegradability, were not investigated. One important safety concern highlighted in this project, which has not been considered previously, is the leakage of intracellular molecules into the extracellular space through the membrane permeabilised by the polymer.

Other methods of intracellular delivery have also shown greater potential in cryopreservation of nucleated cells. Efficient intracellular uptake accompanied by improved protection during freezing was reported for TF-1 haematopoietic cell line, fibroblasts and keratinocytes $[54,135]$ using engineered pores in the membrane, CHO-K1 cell line transfected with trehalose transporter [205], oocytes by microinjections [137], umbilical cord white blood cells using trehalose-loaded liposomes [136], adipose-derived stem cells using trehalose-encapsulated nanoparticles [206] and hepatocytes using modified trehalose molecules [140]. These results are encouraging but the safety of these methods is yet to be comprehensively evaluated, together with other factors relevant to their translation into clinical practice (such as GMP compliance, scalability, cost, etc.). 


\subsubsection{Novel cryopreservation protocol for pancreatic islets}

The current standard in the field for pancreatic islet cryopreservation is Rajotte's step-wise DMSO protocol [73]. While several patients were transplanted with a combination of fresh and cryopreserved islets in the early years of clinical islet transplantation [25], this approach has long been abandoned, reportedly because of the GMP-incompatible presence of FCS in the cryopreserved samples of islets [26].

The ultimate aim of this project was to develop an improved cryopreservation protocol for pancreatic islets. Using the findings from diffusion studies, a protocol was designed which combined cryoprotectants DMSO and trehalose in a novel manner. Since prolonged times are required for small molecules in aqueous solutions to diffuse into the core of the islet, in this protocol islets are loaded with non-toxic trehalose first and DMSO is added in the last step before cryopreservation to minimise its toxic effect.

Trehalose has been previously combined with DMSO or glycerol for cryopreservation of other cell types, in particular haematopoietic cells [131, 132, 204, 186]. Multicellular structures such as canine trachea rings [207], skin sheets [208, 209] or murine embryos [210] were also cryopreserved in a mixture of penetrating cryoprotectant and trehalose. In all these publications, however, trehalose was added at the same time or later than DMSO or glycerol.

Pre-incubation with trehalose followed by addition of penetrating cryoprotectants has not been studied for the protection of multicellular aggregates. However, long pre-incubation with trehalose ( $24 \mathrm{~h}$ and $48 \mathrm{~h}$ ) has been shown to improve DMSO-based cryopreservation protocol in human embryonic stem cells [211]. Importance of prolonged incubation times $(8 \mathrm{~h})$, as well as incubation temperature of $37^{\circ} \mathrm{C}$, for trehalose uptake was recognised by Sun et al. in a study of fibroblast desiccation [212]. In these studies, the incubation time was not needed for diffusion through multicellular structures but rather to allow for passive diffusion of trehalose to the intracellular space.

The only previous report of cryopreservation of pancreatic islet with trehalose is by Beattie et al. [83]. In this paper, viability of human islets cryopreserved with a combination of $2 \mathrm{M}$ DMSO and $300 \mathrm{mM}$ trehalose (added simultaneously) was assessed by total DNA and insulin measurements and compared to fresh islets. They found no differences in these parameters, including in glucose stimulation assays. These islets survived under the mouse kidney capsule for three months after transplantation. However, this protocol was not compared to a DMSO protocol without addition of trehalose and therefore the exact contribution of trehalose is not clear. Importantly, my project investigated an appropriate pre-incubation period with trehalose, as well as generating novel insights into the ideal concentrations of both cryoprotectants. In vitro function of the thawed islets was also evaluated comprehensively, including both insulin secretion and cAMP secretion pathways 
and mitochondrial health and function. Together with viability assays, these assays confirmed the beneficial effect of addition of trehalose to DMSO.

\subsubsection{Potential of antioxidants in islet cryopreservation}

Pancreatic islets are highly sensitive to oxidative stress due to their low expression of antioxidant enzymes [127]. ROS accumulate in pancreatic islets during the process of organ harvesting, islet isolation, culture and cryopreservation and there is extensive evidence that treatment of islets with antioxidants during these procedures improves their viability and glucose secretion [151]. Studies on the use of antioxidants in cryopreservation are limited but beneficial effect has been shown for curcumin [154], ascorbic acid-2 glucoside [213] and taurine [214]. Findings from this project add to this evidence of protective effect of antioxidants in islet cryopreservation by studying salidroside and MitoQ.

Salidroside has been shown to improve cryopreservation of red blood cells [156] but its effect has never been evaluated in pancreatic islets. Here it was shown that salidroside can increase post-thaw viability, particularly if it is added early before cryopreservation.

The antioxidant properties of MitoQ have been studied in many systems and two clinical trials have been performed to study its ability to treat liver dysfunction during hepatitis C [162] and to slow progression of Parkinson's disease [163]. Its protective effect in ischemia reperfusion injury has also been extensively studied in recent years [160, 161]. Here, these studies were extended to demonstrate that MitoQ can reduce oxidative stress during cryopreservation of pancreatic islets.

\subsubsection{Cryopreservation of other multicellular aggregates}

An emerging direction in regenerative and personalised medicine is the use of organoid technology which enables ex vivo culture of human cells in 3D conditions. Organoids contain a stem/progenitor cell population which self-organises and spontaneously differentiates in response to the factors present in the culture medium [215]. This process can in principle be manipulated in order to direct differentiation towards a desired cell type (e.g. pancreatic organoids into $\beta$-cells for treatment of diabetes). This approach has great potential to transform future treatments for patients. Similarly, tumor-derived organoids (tumoroids) can be used for personalised screening of cancer therapies [216].

Regenerative therapies, including organoids, face practical and financial obstacles for their clinical translation in the near future. A potential solution to this is the creation of HLAmatched biobanks containing selected organoid lines which will provide suitably matched cellular therapies for the majority of the population [2]. A key advantage of this approach 
is in its potential to result in almost immediate availability of the treatment, as well as the ability to extensively validate the efficacy and safety of the cellular therapies prior to their use in patients. Cryopreservation represents an essential step for generation of these biobanks.

Despite this pressing need, research in regenerative medicine, including organoid technology, has largely ignored the necessity of cryopreservation for translation of the therapies into clinical practice. While some protocols for cryopreservation of organoids were published [217-219] and specialised media are commercially available, it is not clear how these protocols were developed or what is their efficacy in cryopreservation of the cells. Cryopreservation of organoids encapsulated in alginate beads has been studied more extensively [220,221] but defined and optimised cryopreservation conditions are still unavailable for most organoid types.

Although this project did not focus on organoid cryopreservation, many of its findings are relevant to these and other multicellular aggregates. Firstly, it is clear that defining diffusion rates of cryoprotectants and other substances into multicellular aggregates is key prerequisite for the development of effective freezing protocols. Pancreatic islets differ from organoids and tumoroids in their size and architecture and these parameters must therefore be defined for each organoid type. Methods to study diffusion of small molecules in aqueous solutions were also outlined in this project and it should be possible to use the same methods to define optimal incubation periods for organoids.

The novel cryopreservation protocol developed in this project can also be adapted for the organoids and tumoroids by pre-incubation of cells with non-toxic substances (trehalose/antioxidants) until equilibration, followed by loading of penetrating cryoprotectants and slow-freezing. Again, the specific parameters such as concentrations, incubation times with non-penetrating and penetrating substances or cooling rates must be optimised for each multicellular aggregate.

Cryopreservation of whole organs represents significantly greater complexity than freezing of multicellular aggregates. Nevertheless, this project's findings about loading of nontoxic cryoprotectants have important implications to these systems. However, additional methods have to be developed to ensure homogenous cooling and warming throughout the whole volume, as well as efficient methods for removal of cryoprotectants. 


\subsection{Limitations}

\subsubsection{Assumptions for diffusion studies}

In Chapter 3, diffusion of Hoechst 33342 nuclear dye into the core of pancreatic islets was studied. It was subsequently assumed that these diffusion rates can be used to inform staining protocols with viability dyes FDA and PI and for design of the length of pre-cryopreservation incubation with trehalose or antioxidants. This assumption is supported by the fact that all of these are small molecules dissolved in aqueous solution. However, behaviour of each of the molecules within the islet may differ. Some molecules freely enter the cells (H33342, FDA, MitoQ), while others are likely to remain mostly in the extracellular space (trehalose, salidroside). The extent to which the concentration of these molecules in solution will decrease by irreversible intracellular uptake, binding to cellular structures or through metabolism may also be different to Hoechst 33342.

Attempts to replicate these diffusion studies for FDA, PI and trehalose were unsuccessful. FDA bleaches quickly and studies similar to the one performed with Hoechst 33342 were therefore not possible: no fluorescent signal was detectable by the end of the tissue processing. Conversely, PI does not stain many cells within viable islets and lack of staining therefore does not necessarily imply that the dye has not penetrated to this depth, but rather that the cells at that location are viable. This would therefore make quantification of diffusion into the imaged islet sections inaccurate or impossible.

Direct quantification of the diffusion kinetics of trehalose represents additional challenges. Trehalose is difficult to image without modifications such as conjugation to a fluorescent compound. While this is possible, attachment of fluorescent label such as FITC dramatically changes the characteristics of the trehalose: the fluorescently-labelled trehalose would be significantly larger than unlabelled trehalose and would differs in its ionic charge. Instability of these fluorescent molecules also may lead to additional complexities. This approach was therefore not pursued in this project. Imaging of label-free trehalose using Raman spectroscopy was explored instead. Preliminary experiments with the cell line SAOS-2 showed that quantification of trehalose in cells is possible but further definitive studies are required to explore this approach further.

\subsubsection{Unavailability of islet size data}

As discussed in Chapter 3, in addition to viability, the size of cellular aggregates should also be measured and reported in cryopreservation studies. Development of a reproducible and unbiased method to compare pre-freeze and post-thaw islet size requires additional studies. 
One approach investigate in this project involved handpicking the islets into a Petri dish and imaging of the dish under microscope before transferring them into a cryovial for cryopreservation and repeating the procedure after thawing. While this was technically possible, it delayed the viability staining immediately post-thaw, which increased variability of the results, and it increased the mechanical stress on islets due to multiple handpicking steps. In addition, some islets are necessarily lost during cryopreservation (they may stick to the cryovial, pipette tips etc.) and this skews the analysis because of the small sample size.

A potential solution to this limitation is by increasing the sample size and measuring the average size of a larger number of islets in each group. Because this project used predominantly mouse pancreatic islets, this scale-up was not possible due to the low islet yield from each mouse. However, this approach would be recommended when the results are replicated in larger animal models or in human.

\subsubsection{Statistical analysis of the bi-modally distributed data}

As it was demonstrated in Chapter 5, data obtained from viability measurements of pancreatic islets at $24 \mathrm{~h}$ post-thaw consistently had a bi-modal distribution, wherein a proportion of the islets contained nearly all alive cell and the rest of the islets were fully dead. This distribution was unexpected and its analysis represents specific challenges. Statistical analysis for all islet viability data at $24 \mathrm{~h}$ was nevertheless performed using standard methods with the assumption that this provides suitable approximation of the true means and standard deviations.

This analysis could be improved by creating an appropriate model for the analysis of this bi-modal distribution consisting of two $\beta$ distributions (limited by the interval of viability between 0 and $100 \%$ ) for mostly alive and mostly dead islets. Another, more simplistic way would be to analyse this data as binary (where all mostly viable islets would be assigned value 1 and all nearly dead islets value 0 ).

\subsubsection{Mouse islets as an experimental model}

There are significant differences in the composition and architecture of mouse and human islets [6], as well as their quality after isolation. Because the mouse pancreas is very small, a shorter period of enzymatic digestion is needed and efficient purification is possible, resulting in islets with high viability and purity which are immediately available for use in the laboratory. On the contrary, human pancreases must undergoes prolonged periods of enzymatic digestion and isolated islets are then only partially separated from the exocrine tissue which can release digestive enzymes into the culture. They additionally need to be 
transported from the isolation facility to the research lab, during which the islets can endure further damage.

The experiments in this project were predominantly performed using mouse islets, and infrequent and unpredictable availability of human islet did not enable replication of many of the findings using human cells. This is consistent with the low number of publications on human islet cryopreservation and is a challenge also faced by other research laboratories. However, the clinical translation of the main findings of this project, including diffusion kinetics studies and cryopreservation with trehalose and antioxidants, are critically dependent on their replication using human islets. Of note, in preliminary experiments where cryopreservation of mouse and human islets were compared, the results were similar in both models Nonetheless, the efficacy of the novel protocol in cryopreservation of human pancreatic islets should be examined in future studies.

\subsubsection{Short-term cryopreservation}

All islet samples in this project were thawed within a maximum of four weeks after their cryopreservation. However, if this protocol is used as part of a therapy for diabetes, it must be shown that this method can enable preservation of islets for longer, including up to several years. It has been recently reported that human pancreatic islets from 43 donors were successfully cryopreserved for a mean period of 17.6 years. They maintained their transcriptome profiles and apoptosis levels but had decreased insulin content and decreased ability to revert diabetes in STZ-induced diabetes mouse model in comparison to freshly isolated islets [26].

Such studies are very important for the introduction of cryopreservation into clinical islet transplantation. They are, however, challenging and are best performed in centres which are specialised in human islet isolation and have facilities to maintain a biobank of islets. A critical part of such studies is the consistent and monitored storage of the cryopreserved islets, avoiding unnecessary temperature fluctuations in the liquid nitrogen storage and ensuring that same methods are used for the pre-freeze and post-thaw assessment of islets which will possibly be performed by different researchers due to the long duration of the experiment.

\subsection{Future directions}

\subsubsection{Viability assessment}

The viability assessment method for pancreatic islets proposed in this project consists of prolonged incubation with viability dyes and multi-angle light sheet imaging. This method 
must be validated in future experiments by comparing it to results from other in vitro assays and to the outcome of islet transplantation in mouse models.

An additional key step required for clinical translation and scale-up of the findings of this study is the development of an automated image analysis protocol. Considerations for this were discussed in Chapter 3. Because of the considerable variability between images which caused by the variation in islet size and staining intensity between cells within islet and between islets, advanced thresholding methods will have to be employed. Ideally, machine learning algorithms would be created for this analysis which could then be trained for each laboratory using this technique. Simpler, adaptive thresholding is also possible but the ability to adapt this to the needs of clinical and research lab may be limited.

\subsubsection{Cell cluster survival}

During the development of the new cryopreservation protocol, an interesting phenomenon was observed where the islets became committed to a binary fate during the $24 \mathrm{~h}$ after thawing. A potential mechanism for this has been suggested in Chapter 5.5.1 but the exact nature of this phenomenon should be investigated in future studies. Specifically, experiments should be designed to investigate the specific nature of the intra-cluster communication which, if deficient, leads to cell death. The focus should be not only to identify the adhesion molecules responsible for the critical cell-cell contact but also pathways which are affected when this contact ceases to exist and mechanism which may underlie the requirement for a threshold of cell-cell contact signalling needed to maintain viability of the whole islet. This could also generate insights into the mechanism of islet death in diabetes.

Once the cellular mechanisms have been identified, therapeutics could be designed to target this specific pathway in order to mimic the trophic signals received by islet cells from their neighbouring cells. This approach could provide sufficient time to overcome the initial critical period of cellular stress after thawing and permit cells to remain attached to other surviving cells after cryopreservation.

\subsubsection{Further modifications of the cryopreservation protocol}

In this project it was shown that combinations of different cryoprotectants and additives can lead to superior viability after cryopreservation. Research in additives should therefore continue to target the specific sources of damage during freezing and thawing.

Targeting the aspects of the freezing process which may cause damage to cells is the first approach. In this project DMSO and trehalose have been used but other cryoprotectants are available and have been tested, such as glycerol [79], ethylene glycol [80] or glucose [83]. 
Combining cryoprotectants is also a feasible approach as it could combine the specific protective effects of different cryoprotectants while keeping their concentration to less toxic levels [34].

Recently, ice nucleation agents have been the focus of cryopreservation research. Without interventions, ice nucleation in similar samples does not happen at consistent times and temperatures. Rather, ice nucleation happens randomly upon creation of the crystallisation nucleus. Stochastic nucleation contributes to variability between samples through variability in size and shape of ice crystals, with potential harmful effects on cells. Ice nucleating agents have been developed to assure homogenous and reproducible nucleation at a desired temperature (without supercooling of water) and these agents show great promise in cryopreservation [222].

Another class of molecules which could contribute to better cryopreservation are antifreeze proteins (AFPs). Found in variety of organisms from cold habitats, these proteins allow thermal hysteresis (i.e. lowering the freezing point of water) and once freezing occurs, can bind ice crystals and change their shape. The full potential of these proteins in cryopreservation is yet to be explored but they show promise for sub-zero preservation of tissues [223].

Targeting pathways specific to pancreatic islet survival is another attractive option. As outlined earlier, this will require further research into the mechanisms involved in survival of pancreatic islets. Anti-apoptotic compounds have been developed for other cell types [224, 225]. Other additives can reduce some of the damage leading to apoptosis or necrosis, such as antioxidants. In islet cryopreservation, the tested antioxidants so far include curcumin [154], salidroside and MitoQ (in this study) and all three showed potential to improve the health of islets. Further validation of these antioxidants, as well as investigation of efficacy of other protective compounds, could be investigated in future studies.

Although the potential of permeabilising polymers was shown to be limited in cryopreservation of splenocytes and pancreatic islets in this project, other methods of intracellular delivery are available. Methods suitable for pancreatic islets include in particular membrane phase transition [83] and the use of engineered trehalose molecules [140]. Development of new permeabilising polymers or modification of the current polymers is also possible.

\subsubsection{Validation of the novel cryopreservation protocol}

\section{In vivo testing and replication in human islets}

The novel cryopreservation protocol developed in this project has already been validated in vitro. The efficacy of the cryopreserved islets in reversing hyperglycaemia in diabetic mice 
is yet to be demonstrated. Preliminary in vivo experiments were performed but additional optimisation steps are required, including nephrectomy of the transplanted kidney to ensure the specific effect of the transplanted islets, or glucose challenge of the mice. Genomic and transcriptomic analysis of the islets should also be performed to eliminate any undesired effect of the novel cryopreservation procedure.

Parallel to this, the experiments must be replicated using human pancreatic islets. If these future experiments provide supportive results, the next step would be the transition to pre-clinical testing. In order to do this, standardised procedures must be developed which meet the current Good Manufacturing Practice (GMP) standards and the process must be scaled up to ensure that appropriate dose of the therapy at an affordable cost can be provided. Only after that, the new protocol may proceed to clinical testing and become part of therapy for diabetes.

\section{GMP compliance}

Creation of GMP-compliant protocol requires specialised facilities, trained staff and standardised operating procedures to guarantee sterility and safety of the final therapeutic product. The cells must be characterised throughout the whole process and the final product must meet defined standards. All materials used during the isolation, culture and transport must also be supplied by a certified source and be sterile and of standardised quality [226]. Where possible, xenogenic products should be avoided since their use in therapies requires many additional considerations regarding safety and quality standardisation of the animal product used [227].

Use of human tissue in therapy must conform to additional regulations regarding the selection of suitable donors, testing of the cells and specialised procedures to ensure safe and standardised methods of transport, processing, preservation and storage. Much more attention is also given to documentation of all procedures and location of samples and their final destination to ensure compliance with Human Tissue Authority standards [226].

In this project, consideration was given to procedures and reagents and the possibility of inclusion in a fully GMP-compliant protocol. The only reagent which will have to be substituted or omitted is fetal calf serum due to its coming from a xenogenic source and having largely undefined composition. Unknown components of the serum (including specific components from unborn animals still undergoing development, rather than adults) can then cause undesired effects on cell culture [228]. Human serum albumin is already being used in pre-transplantation islet culture as part of the Edmonton protocol and has also been used in cryopreservation of islets as an alternative to FCS [229]. Substitution of FCS in the final 
protocol, therefore, is not expected to represent a major challenge, although it will lead to an increase in the cost of the final therapeutic product.

A potential safety issue specific to tissue cryopreservation is the risk of infection during liquid nitrogen storage [62] and necessity to ensure monitored maintenance of the liquid nitrogen storage tanks. The proposed protocol uses a liquid nitrogen-free programmable freezer, therefore this issue is avoided for the cooling step. For long-term storage, electrical freezers maintaining temperature of $-150{ }^{\circ} \mathrm{C}$ are also available which are able to overcome the issues related to liquid nitrogen storage.

\section{Scaling up}

Cryopreservation research is typically conducted using small volume cryovials. In this project, all pancreatic islet samples were cryopreserved in $400 \mu \mathrm{L}$ inside $1.8 \mathrm{~mL}$ cryovials. The number of cryopreserved islets was also limited: maximal number of islets cryopreserved in this volume in one cryovial was 250 . For clinical transplantation, these methods must be scaled up to enable cryopreservation of therapeutic dose of islets $(10,000$ IEQ per kilogram body weight [21]) and also to make the final product more affordable.

Cooling and warming of large volumes necessarily entails creation of temperature gradients within the sample, resulting from a slow heat transfer. Unlike in small volume cryopreservation, the cooling of a large volume is not uniform and creation of ice upon nucleation does not happen rapidly. Instead, the volume solidifies in a directional manner (i.e. the cooled surface areas freeze first and the ice then propagates towards the centre of the sample at the rate of the cooling progress) [230]. A related challenge must be overcome during the warming of the sample. It is not possible to achieve rapid warming rates in samples with small surface area to volume ratio. To solve this problem, solutions such as warming devices [231] or nanoparticle warming [232] have been proposed for thawing of large volumes.

Because this topic has not been explored in this project, it is also not clear whether the islet density could be increased without detrimental effect on post-thaw outcome. It has been previously shown with erythrocytes and hepatocytes that increased cell densities cause a 'packing effect' where the post-thaw viability decreases in high cell densities [233, 234]. For small islet densities however, bulk cryopreservation of pancreatic islets in blood freezer bags of volume up to $250 \mathrm{~mL}$ has been explored [235]. It is apparent that scaling up islet cryopreservation is possible and adaptation of the published bulk cryopreservation methods may be possible for the protocol developed in this project. 


\section{Clinical trials}

After scale-up and development of GMP-compatible protocols, the therapy may proceed to clinical trials. Because the new protocol introduced in this thesis uses predominantly agents which have been well characterised and used in the clinical practice or tested in clinical trials, its translation into clinical practice should not present challenges stemming from adverse effect of the reagents. Specifically, DMSO is approved for use in clinical bone marrow transplantation or as part of other therapies and its toxicity has been extensively studied and adverse effects monitored in patients [113]. Trehalose also has a defined safety profile and minimal side effects [118]. The proposed antioxidants have also been reported to be safe: both MitoQ and salidroside-containing extract were successfully tested in clinical trials $[163,159,158]$.

\subsection{Conclusion}

Islet transplantation is currently performed in very limited numbers. Unavailability of longterm storage for islets has impact on the organisation of transplantation, quality control of the tissue and other issues related to multiple-donor transplantation. Although considerable efforts have been made to develop a clinically-applicable method of islet cryopreservation, none have progressed to clinical trials.

In this project, I first critically evaluated the viability assessment protocols for both mononuclear cells and pancreatic islets and identified important limitations of the methods used routinely in laboratory and clinical practice. For viability assessment of mononuclear cells, I propose the creation of a standardised method for use in cryobiology that reports absolute numbers of cells together with viability measurement, ideally in relation to the pre-freeze absolute number of live cells. For pancreatic islets, I demonstrated that current viability staining protocols are suboptimal as they only assess peripheral cells and not the islet core. This may underlie the reported lacked of correlation between islet viability and clinical outcomes.

I then developed a method of cryopreservation of mouse pancreatic islets which combines cryopreservation with DMSO with pre-incubation for $6 \mathrm{~h}$ at $37^{\circ} \mathrm{C}$ with non-toxic cryoprotectant trehalose and antioxidants MitoQ and salidroside. The efficacy of the novel protocol was validated using functional assays, demonstrating preserved function of $\beta$-cell pathways and mitochondrial health. This method, if validated in vivo and using human islets, is potentially suitable for clinical translation.

Investigation of cryopreservation of mononuclear cells using trehalose and permeabilising biopolymers PP-50 and PP-75 revealed limited potential of trehalose alone or with 
permeabilising polymers. Polymers appear to require long incubation times which is not compatible with limited time window for culture of unstimulated mononuclear cells. 



\section{References}

[1] S. Giwa, J. K. Lewis, L. Alvarez, R. Langer, A. E. Roth, G. M. Church, J. F. Markmann, D. H. Sachs, A. Chandraker, J. A. Wertheim, et al. The promise of organ and tissue preservation to transform medicine. Nature Biotechnology, 35(6):530, 2017.

[2] C. J. Taylor, E. M. Bolton, and J. A. Bradley. Immunological considerations for embryonic and induced pluripotent stem cell banking. Philosophical Transactions of the Royal Society of London B: Biological Sciences, 366(1575):2312-2322, 2011.

[3] H. Ichii and C. Ricordi. Current status of islet cell transplantation. Journal of HepatoBiliary-Pancreatic Sciences, 16(2):101-112, 2009.

[4] C. Ionescu-Tirgoviste, P. A. Gagniuc, E. Gubceac, L. Mardare, I. Popescu, S. Dima, and M. Militaru. A 3D map of the islet routes throughout the healthy human pancreas. Scientific Reports, 5:14634, 2015.

[5] X. Wang, R. Misawa, M. C. Zielinski, P. Cowen, J. Jo, V. Periwal, C. Ricordi, A. Khan, J. Szust, J. Shen, et al. Regional differences in islet distribution in the human pancreaspreferential beta-cell loss in the head region in patients with type 2 diabetes. PloS one, 8(6):e67454, 2013.

[6] M. Brissova, M. J. Fowler, W. E. Nicholson, A. Chu, B. Hirshberg, D. M. Harlan, and A. C. Powers. Assessment of human pancreatic islet architecture and composition by laser scanning confocal microscopy. Journal of Histochemistry \& Cytochemistry, 53 (9):1087-1097, 2005.

[7] R. Bertram and M. Pernarowski. Glucose diffusion in pancreatic islets of Langerhans. Biophysical Journal, 74(4):1722-1731, 1998.

[8] G. Kilimnik, J. Jo, V. Periwal, M. C. Zielinski, and M. Hara. Quantification of islet size and architecture. Islets, 4(2):167-172, 2012.

[9] P. E. MacDonald and M. B. Wheeler. Voltage-dependent $\mathrm{K}+$ channels in pancreatic beta cells: role, regulation and potential as therapeutic targets. Diabetologia, 46(8): 1046-1062, 2003.

[10] R. G. Carroll. Elsevier's Integrated Physiology E-Book. Elsevier Health Sciences, 2006.

[11] G. A. Rutter, T. J. Pullen, D. J. Hodson, A. Martinez-Sanchez, K. McLuskey, J. Mottram, I. Delorge, C. M. Figueroa, R. Feil, J. E. Lunn, et al. Pancreatic $\beta$-cell identity, glucose sensing and the control of insulin secretion. Biochemical Journal, 466(2): 203-218, 2015. 
[12] Q. Wang, X. Liang, and S. Wang. Intra-islet glucagon secretion and action in the regulation of glucose homeostasis. Frontiers in physiology, 3:485, 2013.

[13] J. M. Berg, J. L. Tymoczko, L. Stryer, and L. Stryer. Biochemistry, Ed 5th, 2002.

[14] N. Mazzola. Review of current and emerging therapies in type 2 diabetes mellitus. The American Journal of Managed Care, 18(1 Suppl):S17-26, 2012.

[15] U. Boggi, F. Vistoli, F. M. Egidi, P. Marchetti, N. De Lio, V. Perrone, F. Caniglia, S. Signori, M. Barsotti, M. Bernini, et al. Transplantation of the pancreas. Current Diabetes Reports, 12(5):568-579, 2012.

[16] E. A. Ryan, J. R. T. Lakey, R. V. Rajotte, G. S. Korbutt, T. Kin, S. Imes, A. Rabinovitch, J. F. Elliott, D. Bigam, N. M. Kneteman, et al. Clinical outcomes and insulin secretion after islet transplantation with the Edmonton protocol. Diabetes, 50(4):710-719, 2001.

[17] A. M. J. Shapiro, J. R. T. Lakey, E. A. Ryan, G. S. Korbutt, E. Toth, G. L. Warnock, N. M. Kneteman, and R. V. Rajotte. Islet transplantation in seven patients with type 1 diabetes mellitus using a glucocorticoid-free immunosuppressive regimen. New England Journal of Medicine, 343(4):230-238, 2000.

[18] A. M. Frank, C. F. Barker, and J. F. Markmann. Comparison of whole organ pancreas and isolated islet transplantation for type 1 diabetes. Advances in Surgery, 39:137-163, 2005.

[19] C. Ricordi. Lilly Lecture 2002: Islet transplantation: A brave new world. Diabetes, 52(7):1595-1603, 2003.

[20] M. Paget, H. Murray, C. J. Bailey, and R. Downing. Human islet isolation: semiautomated and manual methods. Diabetes and Vascular Disease Research, 4(1):7-12, 2007.

[21] A. M. J. Shapiro, C. Ricordi, B. J. Hering, H. Auchincloss, R. Lindblad, R. P. Robertson, A. Secchi, M. D. Brendel, T. Berney, D. C. Brennan, et al. International trial of the Edmonton protocol for islet transplantation. New England Journal of Medicine, 355(13):1318-1330, 2006.

[22] B. J. Hering, W. R. Clarke, N. D. Bridges, T. L. Eggerman, R. Alejandro, M. D. Bellin, K. Chaloner, C. W. Czarniecki, J. S. Goldstein, L. G. Hunsicker, et al. Phase 3 trial of transplantation of human islets in type 1 diabetes complicated by severe hypoglycemia. Diabetes care, 39(7):1230-1240, 2016.

[23] NHS Blood and Transplant. Annual report on pancreas and islet transplantation, September 2018.

[24] M. R. Rickels, J. Kearns, E. Markmann, M. Palanjian, J. F. Markmann, A. Naji, and M. Kamoun. HLA sensitization in islet transplantation. Clinical transplants, page 413, 2006.

[25] G. L. Warnock, N. M. Kneteman, E. A. Ryan, A. Rabinovitch, and R. V. Rajotte. Long-term follow-up after transplantation of insulin-producing pancreatic islets into patients with type 1 (insulin-dependent) diabetes mellitus. Diabetologia, 35(1):89-95, 1992. 
[26] J. E. Manning Fox, J. Lyon, X. Q. Dai, R. C. Wright, J. Hayward, M. van de Bunt, T. Kin, A. M. James Shapiro, M. I. McCarthy, A. L. Gloyn, et al. Human islet function following 20 years of cryogenic biobanking. Diabetologia, 58(7):1503-1512, 2015.

[27] G. Söderdahl, C. Tammik, M. Remberger, and O. Ringdén. Cadaveric bone marrow and spleen cells for transplantation. Bone Marrow Transplantation, 21(1):79, 1998.

[28] F. J. M. F. Dor, M. L. Ramirez, K. Parmar, E. L. Altman, C. A. Huang, J. D. Down, and D. K. C. Cooper. Primitive hematopoietic cell populations reside in the spleen: Studies in the pig, baboon, and human. Experimental Hematology, 34(11):1573-1582, 2006.

[29] S. Doulatov, F. Notta, E. Laurenti, and J. E. Dick. Hematopoiesis: a human perspective. Cell Stem Cell, 10(2):120-136, 2012.

[30] A. Görgens, S. Radtke, M. Möllmann, M. Cross, J. Dürig, P. A. Horn, and B. Giebel. Revision of the human hematopoietic tree: granulocyte subtypes derive from distinct hematopoietic lineages. Cell Reports, 3(5):1539-1552, 2013.

[31] J. P. Marolleau, L. D. Cortivo, B. Mills, J. P. Fermand, J. M. Miclea, J. P. Lotz, C. Gisselbrecht, and M. Benbunan. Enrichment of peripheral blood CD34+ cells for transplantation using a fully automated immunomagnetic cell selection system and a novel octapeptide releasing agent. Bone Marrow Transplantation, 23(8):819, 1999.

[32] D. Berz, E. M. McCormack, E. S. Winer, G. A. Colvin, and P. J. Quesenberry. Cryopreservation of hematopoietic stem cells. American Journal of Hematology, 82 (6):463-472, 2007.

[33] C. J. Hunt. Cryopreservation of human stem cells for clinical application: a review. Transfusion Medicine and Hemotherapy, 38(2):107-123, 2011.

[34] R. Chian and P. Quinn. Cryobiology: An overview. Fertility Cryopreservation, pages $1-9,2010$.

[35] P. Mazur, S. P. Leibo, and E. H. Y. Chu. A two-factor hypothesis of freezing injury: evidence from Chinese hamster tissue-culture cells. Experimental Cell Research, 71 (2):345-355, 1972.

[36] P. Mazur. Cryobiology: the freezing of biological systems. Science, 168(3934): 939-949, 1970.

[37] J. P. Acker and L. E. McGann. Membrane damage occurs during the formation of intracellular ice. Cryo Letters, 22(4):241-254, 2001.

[38] K. G. M. Brockbank, L. H. Campbell, E. D. Greene, M. C. G. Brockbank, and J. G. Duman. Lessons from nature for preservation of mammalian cells, tissues, and organs. In Vitro Cellular \& Developmental Biology-Animal, 47(3):210-217, 2011.

[39] J. E. Lovelock. The protective action of neutral solutes against haemolysis by freezing and thawing. Biochemical Journal, 56(2):265, 1954. 
[40] G. M. Fahy, B. Wowk, J. Wu, and S. Paynter. Improved vitrification solutions based on the predictability of vitrification solution toxicity. Cryobiology, 48(1):22-35, 2004.

[41] C. Polge, A. U. Smith, and A. S. Parkes. Revival of spermatozoa after vitrification and dehydration at low temperatures. Nature, 164(4172):666, 1949.

[42] J. E. Lovelock and M. W. H. Bishop. Prevention of freezing damage to living cells by dimethyl sulphoxide. Nature, 183(4672):1394, 1959.

[43] J. E. Swain and G. D. Smith. Fertility cryopreservation, chapter 4 (Cryoprotectants), pages 24-38. Cambridge University Press, 2010.

[44] H. T. Meryman. Cryoprotective agents. Cryobiology, 8(2):173-183, 1971.

[45] A. Stolzing, Y. Naaldijk, V. Fedorova, and S. Sethe. Hydroxyethylstarch in cryopreservation-Mechanisms, benefits and problems. Transfusion and Apheresis Science, 46(2):137-147, 2012.

[46] G. M. Fahy. The relevance of cryoprotectant "toxicity" to cryobiology. Cryobiology, 23(1):1-13, 1986.

[47] G. M. Fahy, T. H. Lilley, H. Linsdell, M. S. J. Douglas, and H. T. Meryman. Cryoprotectant toxicity and cryoprotectant toxicity reduction: in search of molecular mechanisms. Cryobiology, 27(3):247-268, 1990.

[48] G. M. Fahy. Cryoprotectant toxicity neutralization. Cryobiology, 60(3):S45-S53, 2010.

[49] P. Windrum, T. C. M. Morris, M. B. Drake, D. Niederwieser, and T. Ruutu. Variation in dimethyl sulfoxide use in stem cell transplantation: a survey of EBMT centres. Bone Marrow Transplantation, 36(7):601, 2005.

[50] A. Smagur, I. Mitrus, S. Giebel, M. Sadus-Wojciechowska, J. Najda, T. Kruzel, T. Czerw, J. Gliwinska, M. Prokop, M. Glowala-Kosinska, et al. Impact of different dimethyl sulphoxide concentrations on cell recovery, viability and clonogenic potential of cryopreserved peripheral blood hematopoietic stem and progenitor cells. Vox Sanguinis, 104(3):240-247, 2013.

[51] A. M. Bakken, O. Bruserud, and J. F. Abrahamsen. No differences in colony formation of peripheral blood stem cells frozen with $5 \%$ or $10 \%$ dimethyl sulfoxide. Journal of Hematotherapy \& Stem Cell Research, 12(3):351-358, 2003.

[52] A. H. Ragab, E. Gilkerson, and M. Myers. Factors in the cryopreservation of bone marrow cells from children with acute lymphocytic leukemia. Cryobiology, 14(2): 125-134, 1977.

[53] P. Halle, O. Tournilhac, W. Knopinska-Posluszny, J. Kanold, P. Gembara, N. Boiret, C. Rapatel, M. Berger, P. Travade, S. Angielski, et al. Uncontrolled-rate freezing and storage at $-80{ }^{\circ} \mathrm{C}$, with only 3.5-percent dmso in cryoprotective solution for 109 autologous peripheral blood progenitor cell transplantations. Transfusion, 41(5): 667-673, 2001. 
[54] S. S. Buchanan, S. A. Gross, J. P. Acker, M. Toner, J. F. Carpenter, and D. W. Pyatt. Cryopreservation of stem cells using trehalose: Evaluation of the method using a human hematopoietic cell line. Stem Cells and Development, 13(3):295-305, 2004.

[55] J. P. Rodrigues, F. H. Paraguassú-Braga, L. Carvalho, E. Abdelhay, L. F. Bouzas, and L. C. Porto. Evaluation of trehalose and sucrose as cryoprotectants for hematopoietic stem cells of umbilical cord blood. Cryobiology, 56(2):144-151, 2008.

[56] S. M. Watt, E. Austin, and S. Armitage. Cryopreservation of hematopoietic stem/progenitor cells for therapeutic use. In Cryopreservation and Freeze-drying Protocols, pages 237-259. Springer, 2007.

[57] B. Balint, Z. Ivanović, M. Petakov, J. Taseski, G. Jovčić, N. Stojanović, and P. Milenković. The cryopreservation protocol optimal for progenitor recovery is not optimal for preservation of marrow repopulating ability. Bone Marrow Transplantation, 23(6):613, 1999.

[58] D. Cilloni, D. Garau, E. Regazzi, G. Sammarelli, B. Savoldo, C. Caramatti, L. Mangoni, V. Rizzoli, and C. Carlo-Stella. Primitive hematopoietic progenitors within mobilized blood are spared by uncontrolled rate freezing. Bone Marrow Transplantation, 23(5):497, 1999.

[59] J. Perez-Oteyza, R. Bornstein, M. Corral, V. Hermosa, A. Alegre, M. Torrabadella, P. Ramos, J. Garcia, J. Odriozola, and J. L. Navarro. Controlled-rate versus uncontrolled-rate cryopreservation of peripheral blood progenitor cells: a prospective multicenter study. Haematologica, 83(11):1001-1005, 1998.

[60] D. D. F. Ma, L. A. Johnson, P. M. Chan, and J. C. Biggs. Factors influencing myeloid stem cell (CFU-C) survival after cryopreservation of human marrow and chronic granulocytic leukemia cells. Cryobiology, 19(1):1-9, 1982.

[61] P. Mazur. Freezing of living cells: mechanisms and implications. American Journal of Physiology-Cell Physiology, 247(3):C125-C142, 1984.

[62] D. Fountain, M. Ralston, N. Higgins, J. B. Gorlin, L. Uhl, C. Wheeler, J. H. Antin, W. H. Churchill, and R. J. Benjamin. Liquid nitrogen freezers: a potential source of microbial contamination of hematopoietic stem cell components. Transfusion, 37(6): 585-591, 1997.

[63] S. D. Rowley, W. I. Bensinger, T. A. Gooley, and C. D. Buckner. Effect of cell concentration on bone marrow and peripheral blood stem cell cryopreservation. Blood, 83(9):2731-2736, 1994.

[64] E. Cabezudo, C. Dalmases, M. Ruz, J. A. Sanchez, C. Torrico, C. Sola, S. Querol, and J. García. Leukapheresis components may be cryopreserved at high cell concentrations without additional loss of HPC function. Transfusion, 40(10):1223-1227, 2000.

[65] F. Beaujean, J. H. Bourhis, C. H. Bayle, H. Jouault, M. Divine, C. Rieux, M. Janvier, C. Le Forestier, and J. L. Pico. Successful cryopreservation of purified autologous CD34+ cells: influence of freezing parameters on cell recovery and engraftment. Bone Marrow Transplantation, 22(11):1091, 1998. 
[66] Y. Katayama, T. Yano, A. Bessho, S. Deguchi, K. Sunami, N. Mahmut, K. Shinagawa, E. Omoto, S. Makino, T. Miyamoto, et al. The effects of a simplified method for cryopreservation and thawing procedures on peripheral blood stem cells. Bone Marrow Transplantation, 19(3):283, 1997.

[67] S. P. Leibo, J. Farrant, P. Mazur, M. G. Hanna Jr, and L. H. Smith. Effects of preezing on marrow stem cell suspensions: Interactions of cooling and warming rates in the presence of pvp, sucrose, or glycerol. Cryobiology, 6(4):315-332, 1970.

[68] C. Röllig, J. Babatz, I. Wagner, A. Maiwald, V. Schwarze, G. Ehninger, and M. Bornhäuser. Thawing of cryopreserved mobilized peripheral blood-comparison between waterbath and dry warming device. Cytotherapy, 4(6):551-555, 2002.

[69] P. Rubinstein, L. Dobrila, R. E. Rosenfield, J. W. Adamson, G. Migliaccio, A. R. Migliaccio, P. E. Taylor, and C. E. Stevens. Processing and cryopreservation of placental/umbilical cord blood for unrelated bone marrow reconstitution. Proceedings of the National Academy of Sciences, 92(22):10119-10122, 1995.

[70] C. G. Perotti, C. D. Fante, G. Viarengo, P. Papa, L. Rocchi, P. Bergamaschi, L. Bellotti, A. Marchesi, and L. Salvaneschi. A new automated cell washer device for thawed cord blood units. Transfusion, 44(6):900-906, 2004.

[71] R. L. Levin, E. G. Cravalho, and C. E. Huggins. Water transport in a cluster of closely packed erythrocytes at subzero temperatures. Cryobiology, 14(5):549-558, 1977.

[72] J. D. Benson, C. T. Benson, and J. K. Critser. Mathematical model formulation and validation of water and solute transport in whole hamster pancreatic islets. Mathematical Biosciences, 254:64-75, 2014.

[73] R. V. Rajotte. Islet cryopreservation protocols. Annals of the New York Academy of Sciences, 875(1):200-207, 1999.

[74] R. V. Rajotte, D. W. Scharp, R. Downing, R. Preston, G. D. Molnar, W. F. Ballinger, and M. H. Greider. Pancreatic islet banking: The transplantation of frozen-thawed rat islets transported between centers. Cryobiology, 18(4):357-369, 1981.

[75] P. Mazur and R. V. Rajotte. Permeability of the 17-day fetal rat pancreas to glycerol and dimethylsulfoxide. Cryobiology, 18(1):1-16, 1981.

[76] M. J. Taylor and M. J. Benton. Interaction of cooling rate, warming rate, and extent of permeation of cryoprotectant in determining survival of isolated rat islets of Langerhans during cryopreservation. Diabetes, 36(1):59-65, 1987.

[77] J. R. T. Lakey, T. J. Anderson, and R. V. Rajotte. Novel approaches to cryopreservation of human pancreatic islets. Transplantation, 72(6):1005-1011, 2001.

[78] M.-A. von Mach, J. Schlosser, M. Weiland, P. J. Feilen, M. Ringel, J. G. Hengstler, L. S. Weilemann, J. Beyer, P. Kann, M. M. Weber, et al. Cryopreservation of islets of Langerhans: Optimization of protocols using rat pancreatic tissue. EXCLI Journal, 2: 6-21, 2003. 
[79] M. J. Taylor, T. J. Duffy, C. J. Hunt, S. R. A. Morgan, and P. J. Davisson. Transplantation and in vitro perifusion of rat islets of Langerhans after slow cooling and warming in the presence of either glycerol or dimethyl sulfoxide. Cryobiology, 20(2):185-204, 1983.

[80] I. Sakonju, Y. Taura, Y. Inayoshi, T. Suzuki, K. Takimoto, M. Nakaichi, and S. Nakama. Cryopreservation of isolated rat islets of langerhans in the presence of ethylene glycol or dimethyl sulfoxide: Evaluation of toxicity and the dynamic pattern of subsequent insulin release in vitro. Cryobiology, 33(3):354-362, 1996.

[81] G. S. Korbutt, J. Ezekowitz, M. M. Rajotte, G. Rayat, and R. V. Rajotte. Successful cryopreservation of rat islets using ethylene glycol. In Transplantation Proceedings, volume 29, page 1983. Elsevier, 1997.

[82] J. R. T. Lakey, P. W. Burridge, and R. V. Rajotte. Cryopreservation of human pancreatic islets. Graft, 5(5):266, 2002.

[83] G. M. Beattie, J. H. Crowe, A. D. Lopez, V. Cirulli, C. Ricordi, and A. Hayek. Trehalose: A cryoprotectant that enhances recovery and preserves function of human pancreatic islets after long-term storage. Diabetes, 46(3):519-523, 1997.

[84] G. G. Kojayan, M. Alexander, D. K. Imagawa, and J. R. T. Lakey. Systematic review of islet cryopreservation. Islets, 10(1):40-49, 2018.

[85] M. M. El-Naggar, F. M. H. Al-Mashat, A. A. Elayat, A. R. M. Sibiany, M. S. M. Ardawi, and M. H. Badawoud. Effect of thawing rate and post-thaw culture on the cryopreserved fetal rat islets: functional and morphological correlation. Life Sciences, 78(17):1925-1932, 2006.

[86] R. V. Rajotte, H. L. Stewart, W. A. G. Voss, T. K. Shnitka, and J. B. Dossetor. Viability studies on frozen-thawed rat islets of Langerhans. Cryobiology, 14(1):116-120, 1977.

[87] J. R. T. Lakey, G. L. Warnock, and R. V. Rajotte. Comparison of methods used for the removal of DMSO following cryopreservation and the development of an automated protocol. Cell Transplantation, 6(2):163-172, 1997.

[88] A. J. F. Reardon, J. A. W. Elliott, and L. E. McGann. Fluorescence as an alternative to light-scatter gating strategies to identify frozen-thawed cells with flow cytometry. Cryobiology, 69(1):91-99, 2014.

[89] H. Yang, A. Pidgorna, M. R. Loutfy, and P. Shuen. Effects of interruptions of controlled-rate freezing on the viability of umbilical cord blood stem cells. Transfusion, 55(1):70-78, 2015.

[90] A. Whitby, L. Whitby, M. Fletcher, J. T. Reilly, D. R. Sutherland, M. Keeney, and D. Barnett. ISHAGE protocol: are we doing it correctly? Cytometry Part B: Clinical Cytometry, 82(1):9-17, 2012.

[91] B. J. Frisch and L. M. Calvi. Hematopoietic stem cell cultures and assays. In Skeletal Development and Repair, pages 315-324. Springer, 2014. 
[92] V. Boyd, O. M. Cholewa, and K. K. Papas. Limitations in the use of fluorescein diacetate/propidium iodide (FDA/PI) and cell permeable nucleic acid stains for viability measurements of isolated islets of Langerhans. Current Trends in Biotechnology and Pharmacy, 2(2):66, 2008.

[93] M. Eckhard, D. Brandhorst, D. Winter, C. Jaeger, H. Jahr, R. G. Bretzel, and M. D. Brendel. The role of current product release criteria for identification of human islet preparations suitable for clinical transplantation. Transplantation Proceedings, 36(5): 1528-1531, 2004.

[94] M. S. Hanson, E. E. Park, M. L. Sears, K. K. Greenwood, J. S. Danobeitia, D. A. Hullett, and L. A. Fernandez. A simplified approach to human islet quality assessment. Transplantation, 89(10):1178, 2010.

[95] K. K. Papas, T. M. Suszynski, and C. K. Colton. Islet assessment for transplantation. Current Opinion in Organ Transplantation, 14(6):674, 2009.

[96] G. C. Weir, P. A. Halban, P. Meda, C. B. Wollheim, L. Orci, and A. E. Renold. Dispersed adult rat pancreatic islet cells in culture: A, B, and D cell function. MetabolismClinical and Experimental, 33(5):447-453, 1984.

[97] C. Ricordi, D. W. R. Gray, B. J. Hering, D. B. Kaufman, G. L. Warnock, N. M. Kneteman, S. P. Lake, N. J. M. London, C. Socci, R. Alejandro, et al. Islet isolation assessment in man and large animals. Acta Diabetologia Latina, 27(3):185-195, 1990.

[98] C. N. Street, J. R. T. Lakey, A. M. J. Shapiro, S. Imes, R. V. Rajotte, E. A. Ryan, J. G. Lyon, T. Kin, J. Avila, T. Tsujimura, et al. Islet graft assessment in the Edmonton protocol: implications for predicting long-term clinical outcome. Diabetes, 53(12): 3107-3114, 2004.

[99] I. R. Sweet, M. Gilbert, R. Jensen, O. Sabek, D. W. Fraga, A. O. Gaber, and J. Reems. Glucose stimulation of cytochrome $\mathrm{C}$ reduction and oxygen consumption as assessment of human islet quality. Transplantation, 80(8):1003-1011, 2005.

[100] K. K. Papas, C. K. Colton, A. Qipo, H. Wu, R. A. Nelson, B. J. Hering, G. C. Weir, and M. Koulmanda. Prediction of marginal mass required for successful islet transplantation. Journal of Investigative Surgery, 23(1):28-34, 2010.

[101] M. Goto, J. Holgersson, M. Kumagai-Braesch, and O. Korsgren. The ADP/ATP ratio: a novel predictive assay for quality assessment of isolated pancreatic islets. American Journal of Transplantation, 6(10):2483-2487, 2006.

[102] Z.-W. Yu and P. J. Quinn. Dimethyl sulphoxide: a review of its applications in cell biology. Bioscience Reports, 14(6):259-281, 1994.

[103] L. E. McGann and M. L. Walterson. Cryoprotection by dimethyl sulfoxide and dimethyl sulfone. Cryobiology, 24(1):11-16, 1987.

[104] T. J. Anchordoguy, C. A. Cecchini, J. H. Crowe, and L. M. Crowe. Insights into the cryoprotective mechanism of dimethyl sulfoxide for phospholipid bilayers. Cryobiology, 28(5):467-473, 1991. 
[105] T. Arakawa, J. F. Carpenter, Y. A. Kita, and J. H. Crowe. The basis for toxicity of certain cryoprotectants: A hypothesis. Cryobiology, 27(4):401-415, 1990.

[106] C. J. Hunt, S. E. Armitage, and D. E. Pegg. Cryopreservation of umbilical cord blood: 2. Tolerance of CD34+ cells to multimolar dimethyl sulphoxide and the effect of cooling rate on recovery after freezing and thawing. Cryobiology, 46(1):76-87, 2003.

[107] E. Pajot-Augy. Comparative effects of cryosolvents on tubulin association, thermal stability, and binding of microtubule-associated proteins. Cryobiology, 30(3):286-298, 1993.

[108] S. J. Baxter and G. H. Lathe. Biochemical effects on kidney of exposure to high concentrations of dimethyl sulphoxide. Biochemical Pharmacology, 20(6):1079-1091, 1971.

[109] T. R. Henderson, R. F. Henderson, and J. L. York. Effects of dimethyl sulfoxide on subunit proteins. Annals of the New York Academy of Sciences, 243(1):38-53, 1975.

[110] I. I. Katkov, M. S. Kim, R. Bajpai, Y. S. Altman, M. Mercola, J. F. Loring, A. V. Terskikh, E. Y. Snyder, and F. Levine. Cryopreservation by slow cooling with DMSO diminished production of Oct-4 pluripotency marker in human embryonic stem cells. Cryobiology, 53(2):194-205, 2006.

[111] M. Iwatani, K. Ikegami, Y. Kremenska, N. Hattori, S. Tanaka, S. Yagi, and K. Shiota. Dimethyl sulfoxide has an impact on epigenetic profile in mouse embryoid body. Stem Cells, 24(11):2549-2556, 2006.

[112] Z. Shu, S. Heimfeld, and D. Gao. Hematopoietic SCT with cryopreserved grafts: adverse reactions after transplantation and cryoprotectant removal before infusion. Bone Marrow Transplantation, 49(4):469, 2014.

[113] M. A. Cox, J. Kastrup, and M. Hrubiško. Historical perspectives and the future of adverse reactions associated with haemopoietic stem cells cryopreserved with dimethyl sulfoxide. Cell and Tissue Banking, 13(2):203-215, 2012.

[114] K. D. Deb. A sweet potion to put embryonic stem cells to sleep. The Scientific World Journal, 9:236-249, 2009.

[115] M. Sola-Penna and J. R. Meyer-Fernandes. Stabilization against thermal inactivation promoted by sugars on enzyme structure and function: why is trehalose more effective than other sugars? Archives of Biochemistry and Biophysics, 360(1):10-14, 1998.

[116] M. del C. Luzardo, F. Amalfa, A. M. Nunez, S. Diaz, A. C. B. De Lopez, and E. A. Disalvo. Effect of trehalose and sucrose on the hydration and dipole potential of lipid bilayers. Biophysical Journal, 78(5):2452-2458, 2000.

[117] J. H. Crowe, L. M. Crowe, A. E. Oliver, N. Tsvetkova, W. Wolkers, and F. Tablin. The trehalose myth revisited: Introduction to a symposium on stabilization of cells in the dry state. Cryobiology, 43(2):89-105, 2001. 
[118] A. B. Richards, S. Krakowka, L. B. Dexter, H. Schmid, A. P. M. Wolterbeek, D. H. Waalkens-Berendsen, A. Shigoyuki, and M. Kurimoto. Trehalose: a review of properties, history of use and human tolerance, and results of multiple safety studies. Food and Chemical Toxicology, 40(7):871-898, 2002.

[119] A. Dahlqvist. Specificity of the human intestinal disaccharidases and implications for hereditary disaccharide intolerance. The Journal of Clinical Investigation, 41(3): 463-470, 1962.

[120] I. A. Murray, K. Coupland, J. A. Smith, I. D. Ansell, and R. G. Long. Intestinal trehalase activity in a UK population: establishing a normal range and the effect of disease. British Journal of Nutrition, 83(3):241-245, 2000.

[121] M. C. Donnamaria, E. I. Howard, and J. R. Grigera. Interaction of water with $\alpha$, $\alpha$-trehalose in solution: molecular dynamics simulation approach. Journal of the Chemical Society, Faraday Transactions, 90(18):2731-2735, 1994.

[122] C. Olsson, H. Jansson, and J. Swenson. The role of trehalose for the stabilization of proteins. The Journal of Physical Chemistry B, 120(20):4723-4731, 2016.

[123] N. K. Jain and I. Roy. Effect of trehalose on protein structure. Protein Science, 18(1): 24-36, 2009.

[124] N. Benaroudj, D. H. Lee, and A. L. Goldberg. Trehalose accumulation during cellular stress protects cells and cellular proteins from damage by oxygen radicals. Journal of Biological Chemistry, 276(26):24261-24267, 2001.

[125] A. L. Lynch, R. Chen, and N. K. H Slater. pH-responsive polymers for trehalose loading and desiccation protection of human red blood cells. Biomaterials, 32(19): 4443-4449, 2011.

[126] K. Ito, A. Hirao, F. Arai, K. Takubo, S. Matsuoka, K. Miyamoto, M. Ohmura, K. Naka, K. Hosokawa, Y. Ikeda, et al. Reactive oxygen species act through p38 MAPK to limit the lifespan of hematopoietic stem cells. Nature Medicine, 12(4):446, 2006.

[127] S. Lenzen, J. Drinkgern, and M. Tiedge. Low antioxidant enzyme gene expression in pancreatic islets compared with various other mouse tissues. Free Radical Biology and Medicine, 20(3):463-466, 1996.

[128] E. Katenz, F. W. R. Vondran, R. Schwartlander, G. Pless, X. Gong, X. Cheng, P. Neuhaus, and I. M. Sauer. Cryopreservation of primary human hepatocytes: the benefit of trehalose as an additional cryoprotective agent. Liver Transplantation, 13 (1):38-45, 2007.

[129] S. Saha, R. Rajamahendran, A. Boediono, C. Sumantri, and T. Suzuki. Viability of bovine blastocysts obtained after 7, 8 or 9 days of culture in vitro following vitrification and one-step rehydration. Theriogenology, 46(2):331-343, 1996.

[130] C. Scheinkönig, S. Kappicht, H. J. Kolb, and M. Schleuning. Adoption of long-term cultures to evaluate the cryoprotective potential of trehalose for freezing hematopoietic stem cells. Bone Marrow Transplantation, 34(6):531, 2004. 
[131] X. B. Zhang, K. Li, K. H. Yau, K. S. Tsang, T. F. Fok, C. K. Li, S. M. Lee, and P. M. P. Yuen. Trehalose ameliorates the cryopreservation of cord blood in a preclinical system and increases the recovery of CFUs, long-term culture-initiating cells, and nonobese diabetic-SCID repopulating cells. Transfusion, 43(2):265-272, 2003.

[132] L. S. Limaye and V. P. Kale. Cryopreservation of human hematopoietic cells with membrane stabilizers and bioantioxidants as additives in the conventional freezing medium. Journal of Hematotherapy \& Stem Cell Research, 10(5):709-718, 2001.

[133] W. Chen, Z. Shu, D. Gao, and A. Q. Shen. Sensing and sensibility: Single-islet-based quality control assay of cryopreserved pancreatic islets with functionalized hydrogel microcapsules. Advanced Healthcare Materials, 5(2):223-231, 2016.

[134] H. Noguchi, B. Naziruddin, N. Onaca, A. Jackson, M. Shimoda, T. Ikemoto, Y. Fujita, N. Kobayashi, M. F. Levy, and S. Matsumoto. Comparison of modified Celsior solution and M-Kyoto solution for pancreas preservation in human islet isolation. Cell Transplantation, 19(6-7):751-758, 2010.

[135] A. Eroglu, M. J. Russo, R. Bieganski, A. Fowler, S. Cheley, H. Bayley, and M. Toner. Intracellular trehalose improves the survival of cryopreserved mammalian cells. Nature Biotechnology, 18(2):163, 2000.

[136] J. P. R. Motta, F. H. Paraguassú-Braga, L. F. Bouzas, and L. C. Porto. Evaluation of intracellular and extracellular trehalose as a cryoprotectant of stem cells obtained from umbilical cord blood. Cryobiology, 68(3):343-348, 2014.

[137] A. Eroglu, M. Toner, and T. L. Toth. Beneficial effect of microinjected trehalose on the cryosurvival of human oocytes. Fertility and Sterility, 77(1):152-158, 2002.

[138] J. C. Mohr, J. J. de Pablo, and S. P. Palecek. Electroporation of human embryonic stem cells: Small and macromolecule loading and DNA transfection. Biotechnology Progress, 22(3):825-834, 2006.

[139] I. Puhlev, N. Guo, D. R. Brown, and F. Levine. Desiccation tolerance in human cells. Cryobiology, 42(3):207-217, 2001.

[140] A. Abazari, L. G. Meimetis, G. Budin, S. S. Bale, R. Weissleder, and M. Toner. Engineered trehalose permeable to mammalian cells. PLoS One, 10(6):e0130323, 2015.

[141] A. L. Lynch, R. Chen, P. J. Dominowski, E. Y. Shalaev, R. J. Yancey Jr, and N. K. H. Slater. Biopolymer mediated trehalose uptake for enhanced erythrocyte cryosurvival. Biomaterials, 31(23):6096-6103, 2010.

[142] D. M. C. Sharp, A. Picken, T. J. Morris, C. J. Hewitt, K. Coopman, and N. K. H. Slater. Amphipathic polymer-mediated uptake of trehalose for dimethyl sulfoxide-free human cell cryopreservation. Cryobiology, 67(3):305-311, 2013.

[143] S. Stewart and X. He. Intracellular delivery of trehalose for cell banking. Langmuir, 2018. 
[144] R. Chen, M. E. Eccleston, Z. Yue, and N. K. H. Slater. Synthesis and pH-responsive properties of pseudo-peptides containing hydrophobic amino acid grafts. Journal of Materials Chemistry, 19(24):4217-4224, 2009.

[145] M. E. Eccleston, M. Kuiper, F. M. Gilchrist, and N. K. H. Slater. pH-responsive pseudo-peptides for cell membrane disruption. Journal of Controlled Release, 69(2): 297-307, 2000.

[146] S. A. Mercado and N. K. H. Slater. Increased cryosurvival of osteosarcoma cells using an amphipathic $\mathrm{pH}$-responsive polymer for trehalose uptake. Cryobiology, 73 (2):175-180, 2016.

[147] P. Jezek, M. Jaburek, and L. Plecita-Hlavata. Contribution of oxidative stress and impaired biogenesis of pancreatic $\beta$-cells to type 2 diabetes. Antioxidants \& Redox Signaling, 2018.

[148] S. Orrenius, V. Gogvadze, and B. Zhivotovsky. Mitochondrial oxidative stress: implications for cell death. Annual Review of Pharmacology and Toxicology, 47:143-183, 2007.

[149] C. Tatone, G. Di Emidio, M. Vento, R. Ciriminna, and P. G. Artini. Cryopreservation and oxidative stress in reproductive cells. Gynecological Endocrinology, 26(8):563$567,2010$.

[150] M. E. Garcez, C. dos Santos Branco, L. V. Lara, F. F. Pasqualotto, and M. Salvador. Effects of resveratrol supplementation on cryopreservation medium of human semen. Fertility and sterility, 94(6):2118-2121, 2010.

[151] S. S. M. S. Monfared, B. Larijani, and M. Abdollahi. Islet transplantation and antioxidant management: a comprehensive review. World Journal of Gastroenterology, 15(10):1153, 2009.

[152] Y. Tanaka, C. E. Gleason, P. O. T. Tran, J. S. Harmon, and R. P. Robertson. Prevention of glucose toxicity in HIT-T15 cells and Zucker diabetic fatty rats by antioxidants. Proceedings of the National Academy of Sciences, 96(19):10857-10862, 1999.

[153] Y. S. Oh. Plant-derived compounds targeting pancreatic beta cells for the treatment of diabetes. Evidence-Based Complementary and Alternative Medicine, 2015, 2015.

[154] M. Kanitkar and R. R. Bhonde. Curcumin treatment enhances islet recovery by induction of heat shock response proteins, Hsp70 and heme oxygenase-1, during cryopreservation. Life Sciences, 82(3-4):182-189, 2008.

[155] E. W. Qian, D. T. Ge, and S.-K. Kong. Salidroside protects human erythrocytes against hydrogen peroxide-induced apoptosis. Journal of Natural Products, 75(4):531-537, 2012.

[156] N. A. S. Al-Otaibi, N. K. H. Slater, and H. Rahmoune. Salidroside as a novel protective agent to improve red blood cell cryopreservation. PLoS One, 11(9):e0162748, 2016. 
[157] N. A. S. Al-Otaibi, J. S. Cassoli, D. Martins-de Souza, N. K. H. Slater, and H. Rahmoune. Human Leukaemia cells (HL-60) proteomic and biological signatures underpinning cryo-damage are differentially modulated by novel cryo-additives. GigaScience, 2018.

[158] V. Darbinyan, G. Aslanyan, E. Amroyan, E. Gabrielyan, C. Malmström, and A. Panossian. Clinical trial of Rhodiola rosea L. extract SHR-5 in the treatment of mild to moderate depression. Nordic Journal of Psychiatry, 61(5):343-348, 2007.

[159] R. A. J. Smith and M. P. Murphy. Animal and human studies with the mitochondriatargeted antioxidant MitoQ. Annals of the New York Academy of Sciences, 1201(1): 96-103, 2010.

[160] A. J. Dare, A. Logan, T. A. Prime, S. Rogatti, M. Goddard, E. M. Bolton, J. A. Bradley, G. J. Pettigrew, M. P. Murphy, and K. Saeb-Parsy. The mitochondria-targeted anti-oxidant MitoQ decreases ischemia-reperfusion injury in a murine syngeneic heart transplant model. The Journal of Heart and Lung Transplantation, 34(11):1471-1480, 2015 .

[161] A. J. Dare, E. A. Bolton, G. J. Pettigrew, J. A. Bradley, K. Saeb-Parsy, and M. P. Murphy. Protection against renal ischemia-reperfusion injury in vivo by the mitochondria targeted antioxidant MitoQ. Redox Biology, 5:163-168, 2015.

[162] E. J. Gane, F. Weilert, D. W. Orr, G. F. Keogh, M. Gibson, M. M. Lockhart, C. M. Frampton, K. M. Taylor, R. A. J. Smith, and M. P. Murphy. The mitochondria-targeted anti-oxidant mitoquinone decreases liver damage in a phase ii study of hepatitis $\mathrm{C}$ patients. Liver International, 30(7):1019-1026, 2010.

[163] B. J. Snow, F. L. Rolfe, M. M. Lockhart, C. M. Frampton, J. D. O’Sullivan, V. Fung, R. A. J. Smith, M. P. Murphy, K. M. Taylor, and Protect Study Group. A double-blind, placebo-controlled study to assess the mitochondria-targeted antioxidant MitoQ as a disease-modifying therapy in Parkinson's disease. Movement Disorders, 25(11): 1670-1674, 2010.

[164] S. Lim, M. A. Rashid, M. Jang, Y. Kim, H. Won, J. Lee, J. Woo, Young S. Kim, M. P. Murphy, L. Ali, et al. Mitochondria-targeted antioxidants protect pancreatic $\beta$-cells against oxidative stress and improve insulin secretion in glucotoxicity and glucolipotoxicity. Cellular Physiology and Biochemistry, 28(5):873-886, 2011.

[165] L. Fang, C. Bai, Y. Chen, J. Dai, Y. Xiang, X. Ji, C. Huang, and Q. Dong. Inhibition of ROS production through mitochondria-targeted antioxidant and mitochondrial uncoupling increases post-thaw sperm viability in yellow catfish. Cryobiology, 69(3): 386-393, 2014.

[166] Y. Sui, Q. Fan, B. Wang, J. Wang, and Q. Chang. Ice-free cryopreservation of heart valve tissue: The effect of adding MitoQ to a VS83 formulation and its influence on mitochondrial dynamics. Cryobiology, 81:153, 2018.

[167] B. Machalinski, J. Kijowski, W. Marlicz, A. Gontarewicz, M. Markiewski, M. Paczkowski, A. Kopkowski, M. Majka, M. Ostrowski, and M. Z. Ratajczak. Heparinized cadaveric organ donors (HCOD)-A potential source of hematopoietic cells for transplantation and gene therapy. Transplantation, 71(7):1003-1007, 2001. 
[168] L. D. Shultz, B. L. Lyons, L. M. Burzenski, B. Gott, X. Chen, S. Chaleff, M. Kotb, S. D. Gillies, M. King, J. Mangada, et al. Human lymphoid and myeloid cell development in NOD/LtSz-scid IL2R $\gamma$ null mice engrafted with mobilized human hemopoietic stem cells. The Journal of Immunology, 174(10):6477-6489, 2005.

[169] B. L. Strehler. Adenosine-5-triphosphate and creatine phosphate determination with luciferase. In Methods of Enzymatic Analysis (Second Edition), Volume 4, pages 2112-2126. Elsevier, 1974.

[170] A. M. Petrunkina and R. A. P. Harrison. Mathematical analysis of mis-estimation of cell subsets in flow cytometry: viability staining revisited. Journal of Immunological Methods, 368(1-2):71-79, 2011.

[171] J. Crank. The Mathematics of Diffusion: 2nd Ed. Clarendon Press, 1975.

[172] R. B. Bird. Transport phenomena. Applied Mechanics Reviews, 55(1):R1-R4, 2002.

[173] R. Notman, W. K. den Otter, M. G. Noro, W. J. Briels, and J. Anwar. The permeability enhancing mechanism of DMSO in ceramide bilayers simulated by molecular dynamics. Biophysical Journal, 93(6):2056-2068, 2007.

[174] H. Komatsu, C. Cook, C.-H. Wang, L. Medrano, H. Lin, F. Kandeel, Y.-C. Tai, and Y. Mullen. Oxygen environment and islet size are the primary limiting factors of isolated pancreatic islet survival. PLoS One, 12(8):e0183780, 2017.

[175] V. J. Pansare, S. Hejazi, W. J. Faenza, and R. K. Prud'homme. Review of longwavelength optical and NIR imaging materials: contrast agents, fluorophores, and multifunctional nano carriers. Chemistry of Materials, 24(5):812-827, 2012.

[176] E. Ilegems, P. P. Van Krieken, P. K. Edlund, A. Dicker, T. Alanentalo, M. Eriksson, S. Mandic, U. Ahlgren, and P.-O. Berggren. Light scattering as an intrinsic indicator for pancreatic islet cell mass and secretion. Scientific Reports, 5:10740, 2015.

[177] L. M. Sasnoor, V. P. Kale, and L. S. Limaye. Supplementation of conventional freezing medium with a combination of catalase and trehalose results in better protection of surface molecules and functionality of hematopoietic cells. Journal of Hematotherapy \& Stem Cell Research, 12(5):553-564, 2003.

[178] S. Alencar, M. Garnica, R. R. Luiz, C. M. Nogueira, R. Borojevic, A. Maiolino, and H. S. Dutra. Cryopreservation of peripheral blood stem cell: The influence of cell concentration on cellular and hematopoietic recovery. Transfusion, 50(11):2402-2412, 2010 .

[179] Y. Kawano, C. L. Lee, T. Watanabe, T. Abe, H. Suzuya, Y. Okamoto, A. Makimoto, R. Nakagawa, H. Watanabe, and Y. Takaue. Cryopreservation of mobilized blood stem cells at a higher cell concentration without the use of a programmed freezer. Annals of Hematology, 83(1):50-54, 2004.

[180] P. J. Stiff, A. J. Murgo, C. G. Zaroulis, M. F. DeRisi, and B. D. Clarkson. Unfractionated human marrow cell cryopreservation using dimethylsulfoxide and hydroxyethyl starch. Cryobiology, 20(1):17-24, 1983. 
[181] G. Grilli, A. Porcellini, and G. Lucarelli. Role of serum on cryopreservation and subsequent viability of mouse bone marrow hemopoietic stem cells. Cryobiology, 17 (5):516-520, 1980.

[182] A. L. Lynch and N. K. H. Slater. Influence of intracellular trehalose concentration and pre-freeze cell volume on the cryosurvival of rapidly frozen human erythrocytes. Cryobiology, 63(1):26-31, 2011.

[183] M. M. Gedde, E. Yang, and W. H. Huestis. Shape response of human erythrocytes to altered cell pH. Blood, 86(4):1595-1599, 1995.

[184] A. Abazari, N. Chakraborty, S. Hand, A. Aksan, and M. Toner. A Raman microspectroscopy study of water and trehalose in spin-dried cells. Biophysical Journal, 107 (10):2253-2262, 2014.

[185] S. Chen, S. Wang, M. Kopytynski, M. Bachelet, and R. Chen. Membrane-anchoring, comb-like pseudopeptides for efficient, $\mathrm{pH}$-mediated membrane destabilization and intracellular delivery. ACS Applied Materials \& Interfaces, 9(9):8021-8029, 2017.

[186] S. Mantri, S. Kanungo, and P. C. Mohapatra. Cryoprotective effect of disaccharides on cord blood stem cells with minimal use of DMSO. Indian Journal of Hematology and Blood Transfusion, 31(2):206-212, 2015.

[187] H. Tomoda, Y. Kishimoto, and Y. C. Lee. Temperature effect on endocytosis and exocytosis by rabbit alveolar macrophages. Journal of Biological Chemistry, 264(26): 15445-15450, 1989.

[188] O. Cabrera, D. M. Berman, N. S. Kenyon, C. Ricordi, P.-O. Berggren, and A. Caicedo. The unique cytoarchitecture of human pancreatic islets has implications for islet cell function. Proceedings of the National Academy of Sciences, 103(7):2334-2339, 2006.

[189] C. Benner, T. van der Meulen, E. Cacéres, K. Tigyi, C. J. Donaldson, and M. O. Huising. The transcriptional landscape of mouse beta cells compared to human beta cells reveals notable species differences in long non-coding rna and protein-coding gene expression. BMC Genomics, 15(1):620, 2014.

[190] R. V. Rajotte, G. L. Warnock, and N. M. Kneteman. Methods of islet cryopreservation. Pancreatic Islet Transplantation, pages 124-131, 1992.

[191] J. Foreman, H. Moriya, and M. J. Taylor. Effect of cooling rate and its interaction with pre-freeze and post-thaw tissue culture on the in vitro and in vivo function of cryopreserved pancreatic islets. Transplant International, 6(4):191-200, 1993.

[192] R. V. Rajotte, G. L. Warnock, L. C. Bruch, and A. W. Procyshyn. Transplantation of cryopreserved and fresh rat islets and canine pancreatic fragments: comparison of cryopreservation protocols. Cryobiology, 20(2):169-184, 1983.

[193] R. V. Rajotte and P. Mazur. Survival of frozen-thawed fetal rat pancreases as a function of the permeation of dimethylsulfoxide and glycerol, warming rate, and fetal age. Cryobiology, 18(1):17-31, 1981. 
[194] H. Ramachandran, J. Laux, I. Moldovan, R. Caspell, P. V. Lehmann, and R. A. Subbramanian. Optimal thawing of cryopreserved peripheral blood mononuclear cells for use in high-throughput human immune monitoring studies. Cells, 1(3):313-324, 2012.

[195] C. Yuan, J. Gao, J. Guo, L. Bai, C. Marshall, Z. Cai, L. Wang, and M. Xiao. Dimethyl sulfoxide damages mitochondrial integrity and membrane potential in cultured astrocytes. PLoS One, 9(9):e107447, 2014.

[196] I. Sadowska-Bartosz, A. Pączka, M. Mołoń, and G. Bartosz. Dimethyl sulfoxide induces oxidative stress in the yeast Saccharomyces cerevisiae. FEMS Yeast Research, 13(8):820-830, 2013.

[197] X.-G. Zhang, Y.-H. Wang, C. Han, S. Hu, L.-Q. Wang, and J.-H. Hu. Effects of trehalose supplementation on cell viability and oxidative stress variables in frozenthawed bovine calf testicular tissue. Cryobiology, 70(3):246-252, 2015.

[198] K. Rashid and P. C. Sil. Curcumin enhances recovery of pancreatic islets from cellular stress induced inflammation and apoptosis in diabetic rats. Toxicology and Applied Pharmacology, 282(3):297-310, 2015.

[199] R. Echigo, N. Shimohata, K. Karatsu, F. Yano, Y. Kayasuga-Kariya, A. Fujisawa, T. Ohto, Y. Kita, M. Nakamura, S. Suzuki, et al. Trehalose treatment suppresses inflammation, oxidative stress, and vasospasm induced by experimental subarachnoid hemorrhage. Journal of Translational Medicine, 10(1):80, 2012.

[200] S. M. Frisch and H. Francis. Disruption of epithelial cell-matrix interactions induces apoptosis. The Journal of Cell Biology, 124(4):619-626, 1994.

[201] P. Paoli, E. Giannoni, and P. Chiarugi. Anoikis molecular pathways and its role in cancer progression. Biochimica et Biophysica Acta (BBA)-Molecular Cell Research, 1833(12):3481-3498, 2013.

[202] R. E. Durand. Use of Hoechst 33342 for cell selection from multicell systems. Journal of Histochemistry \& Cytochemistry, 30(2):117-122, 1982.

[203] D. Martinetti, C. Colarossi, S. Buccheri, G. Denti, L. Memeo, and L. Vicari. Effect of trehalose on cryopreservation of pure peripheral blood stem cells. Biomedical Reports, 6(3):314-318, 2017.

[204] J. P. R. Motta, B. E. Gomes, L. F. Bouzas, F. H. Paraguassu-Braga, and L. C. Porto. Evaluations of bioantioxidants in cryopreservation of umbilical cord blood using natural cryoprotectants and low concentrations of dimethylsulfoxide. Cryobiology, 60 (3):301-307, 2010.

[205] T. Uchida, M. Furukawa, T. Kikawada, K. Yamazaki, and K. Gohara. Intracellular trehalose via transporter TRET1 as a method to cryoprotect CHO-K1 cells. Cryobiology, 77:50-57, 2017. 
[206] W. Rao, H. Huang, H. Wang, S. Zhao, J. Dumbleton, G. Zhao, and X. He. Nanoparticlemediated intracellular delivery enables cryopreservation of human adipose-derived stem cells using trehalose as the sole cryoprotectant. ACS Applied Materials \& Interfaces, 7(8):5017-5028, 2015.

[207] H. Yokomise, K. Inui, H. Wada, S. Hasegawa, N. Ohno, and S. Hitomi. Reliable cryopreservation of trachea for one month in a new trehalose solution. The Journal of Thoracic and Cardiovascular Surgery, 110(2):382-385, 1995.

[208] F. Chen, W. Zhang, W. Wu, Y. Jin, L. Cen, J. D. Kretlow, W. Gao, Z. Dai, J. Wang, G. Zhou, et al. Cryopreservation of tissue-engineered epithelial sheets in trehalose. Biomaterials, 32(33):8426-8435, 2011.

[209] G. Erdag, A. Eroglu, J. R. Morgan, and M. Toner. Cryopreservation of fetal skin is improved by extracellular trehalose. Cryobiology, 44(3):218-228, 2002.

[210] T. E. Honadel and G. J. Killian. Cryopreservation of murine embryos with trehalose and glycerol. Cryobiology, 25(4):331-337, 1988.

[211] L. Ji, J. J. de Pablo, and S. P. Palecek. Cryopreservation of adherent human embryonic stem cells. Biotechnology and Bioengineering, 88(3):299-312, 2004.

[212] M. Sun, M. Jiang, J. Cui, W. Liu, L. Yin, C. Xu, Q. Wei, X. Yan, and F. Chen. A novel approach for the cryodesiccated preservation of tissue-engineered skin substitutes with trehalose. Materials Science and Engineering: C, 60:60-66, 2016.

[213] T. Arata, T. Okitsu, T. Fukazawa, H. Ikeda, K. Kobayashi, C. Yong, Y. Kosaka, M. Narushima, J. Matsuoka, I. Yamamoto, et al. Maintenance of glucose-sensitive insulin secretion of cryopreserved human islets with University of Wisconsin solution and ascorbic acid-2 glucoside. Artificial Organs, 28(6):529-536, 2004.

[214] A. A. Hardikar, M. V. Risbud, C. Remacle, B. Reusens, J. J. Hoet, and R. R. Bhonde. Islet cryopreservation: improved recovery following taurine pretreatment. Cell Transplantation, 10(3):247-253, 2001.

[215] M. Huch, J. A. Knoblich, M. P. Lutolf, and A. Martinez-Arias. The hope and the hype of organoid research. Development, 144(6):938-941, 2017.

[216] L. Broutier, G. Mastrogiovanni, M. M. A. Verstegen, H. E. Francies, L. M. Gavarró, C. R. Bradshaw, G. E. Allen, R. Arnes-Benito, O. Sidorova, M. P. Gaspersz, et al. Human primary liver cancer-derived organoid cultures for disease modeling and drug screening. Nature Medicine, 23(12):1424, 2017.

[217] L. Broutier, A. Andersson-Rolf, C. J. Hindley, S. F. Boj, H. Clevers, B. Koo, and M. Huch. Culture and establishment of self-renewing human and mouse adult liver and pancreas 3D organoids and their genetic manipulation. Nature Protocols, 11(9): 1724, 2016.

[218] H. Miyoshi and T. S. Stappenbeck. In vitro expansion and genetic modification of gastrointestinal stem cells in spheroid culture. Nature Protocols, 8(12):2471, 2013. 
[219] X. Xue and Y. M. Shah. In vitro organoid culture of primary mouse colon tumors. Journal of Visualized Experiments: JoVE, 1(75), 2013.

[220] P. Kilbride, S. Lamb, S. Gibbons, J. Bundy, E. Erro, C. Selden, B. Fuller, and J. Morris. Cryopreservation and re-culture of a 2.3 litre biomass for use in a bioartificial liver device. PLoS One, 12(8):e0183385, 2017.

[221] Y.-C. Lu, D.-J. Fu, D. An, A. Chiu, R. Schwartz, A. Y. Nikitin, and M. Ma. Scalable production and cryostorage of organoids using core-shell decoupled hydrogel capsules. Advanced Biosystems, 1(12):1700165, 2017.

[222] G. J. Morris and E. Acton. Controlled ice nucleation in cryopreservation-a review. Cryobiology, 66(2):85-92, 2013.

[223] J. Barrett. Thermal hysteresis proteins. The International Journal of Biochemistry \& Cell Biology, 33(2):105-117, 2001.

[224] G. Wu, L. D. Tomei, I. C. Bathurst, F. Zhang, C. B. Hong, C. J. Issel, A. Columbano, R. K. Salley, and S. Chien. Antiapoptotic compound to enhance hypothermic liver preservation1. Transplantation, 63(6):803-809, 1997.

[225] A. H. Salehi, S. J. Morris, W.-C. Ho, K. M. Dickson, G. Doucet, S. Milutinovic, J. Durkin, J. W. Gillard, and P. A. Barker. AEG3482 is an antiapoptotic compound that inhibits Jun kinase activity and cell death through induced expression of heat shock protein 70. Chemistry \& Biology, 13(2):213-223, 2006.

[226] L. Tsang. New areas: Cell therapy and tissue engineering products-technical, legal and regulatory considerations. Medicines from Animal Cell Culture, pages 637-649, 2007.

[227] R. Festen. Understanding animal sera: Considerations for use in the production of biological. Medicines from Animal Cell Culture, page 45, 2007.

[228] J. van der Valk, K. Bieback, C. Buta, B. Cochrane, W. G. Dirks, J. Fu, J. J. Hickman, C. Hohensee, R. Kolar, M. Liebsch, et al. Fetal bovine serum (FBS): past-presentfuture. ALTEX-Alternatives to animal experimentation, 35(1):99-118, 2018.

[229] P. M. Miranda, V. Mohan, S. Ganthimathy, R. M. Anjana, S. Gunasekaran, V. Thiagarajan, T. A. Churchill, T. Kin, A. M. J. Shapiro, and J. R. T. Lakey. Human islet mass, morphology, and survival after cryopreservation using the Edmonton protocol. Islets, 5(5):188-195, 2013.

[230] P. Kilbride, G. J. Morris, S. Milne, B. Fuller, J. Skepper, and C. Selden. A scale down process for the development of large volume cryopreservation. Cryobiology, 69(3): 367-375, 2014.

[231] P. Kilbride, S. Lamb, S. Milne, S. Gibbons, E. Erro, J. Bundy, C. Selden, B. Fuller, and J. Morris. Spatial considerations during cryopreservation of a large volume sample. Cryobiology, 73(1):47-54, 2016. 
[232] N. Manuchehrabadi, Z. Gao, J. Zhang, H. L. Ring, Q. Shao, F. Liu, M. McDermott, A. Fok, Y. Rabin, K. G. M. Brockbank, et al. Improved tissue cryopreservation using inductive heating of magnetic nanoparticles. Science Translational Medicine, 9(379): eaah4586, 2017.

[233] D. E. Pegg, M. P. Diaper, C. J. Hunt, et al. The effect of cooling rate and warming rate on the packing effect in human erythrocytes frozen and thawed in the presence of $2 \mathrm{M}$ glycerol. Cryobiology, 21(5):491-502, 1984.

[234] W. De Loecker, V. A. Koptelov, V. I. Grischenko, and P. De Loecker. Effects of cell concentration on viability and metabolic activity during cryopreservation. Cryobiology, 37(2):103-109, 1998.

[235] M. Miyamoto, A. N. Balamurugan, Y. Nozawa, T. Sakurai, B. Xu, S. Yoshimura, T. Tanaka, T. Tohyama, J. Miyakoshi, and K. Inoue. Development of a cryopreservation procedure employing a freezer bag for pancreatic islets using a newly developed cryoprotectant. Cell Transplantation, 10(4-5):363-371, 2001.

[236] N. J. Hart and A. C. Powers. Use of human islets to understand islet biology and diabetes: progress, challenges and suggestions. Diabetologia, 62(2):212-222, 2019.

[237] N. Bauer, A. Fonesca, M. Florek, D. Freund, J. Jászai, M. Bornhäuser, C. A. Fargeas, and D. Corbeil. New insights into the cell biology of hematopoietic progenitors by studying prominin-1 (CD133). Cells Tissues Organs, 188(1-2):127-138, 2008.

[238] M. C. Deeds, J. M. Anderson, A. S. Armstrong, D. A. Gastineau, H. J. Hiddinga, A. Jahangir, N. L. Eberhardt, and Y. C. Kudva. Single dose streptozotocin-induced diabetes: Considerations for study design in islet transplantation models. Laboratory Animals, 45(3):131-140, 2011.

[239] M. J. Lima, K. R. Muir, H. M. Docherty, N. W. A. McGowan, S. Forbes, Y. Heremans, H. Heimberg, J. Casey, and K. Docherty. Generation of functional beta-like cells from human exocrine pancreas. PLoS One, 11(5):e0156204, 2016.

[240] The Jackson Laboratory. NSG STZ-induced diabetes data, December 2018. URL https://www.jax.org/jax-mice-and-services/find-and-order-jax-mice/ surgical-and-preconditioning-services/stz-induced-diabetes/stz-nsg-data\#.

[241] Y. Togashi, J. Shirakawa, T. Okuyama, S. Yamazaki, M. Kyohara, A. Miyazawa, T. Suzuki, M. Hamada, and Y. Terauchi. Evaluation of the appropriateness of using glucometers for measuring the blood glucose levels in mice. Scientific Reports, 6: 25465, 2016. 



\section{Appendix A}

\section{Donor demographics}

\section{A.1 Demographic information of deceased human donors}

Table A.1 Demographics of deceased human organ donors used in experiments

\begin{tabular}{cccccccccc}
\hline Donor code & Donor type & Age & Gender & & Donor code & Donor type & Age & Gender \\
\cline { 1 - 6 } D1 & DCD & 63 & F & & D18 & DBD & 36 & F \\
D2 & DBD & 49 & M & & D19 & DBD & 58 & F \\
D3 & DBD & 47 & F & D20 & DBD & 54 & M \\
D4 & DBD & 54 & F & D21 & DCD & 46 & M \\
D5 & DBD & 20 & F & D22 & DBD & 27 & F \\
D6 & DCD & 39 & M & D23 & DBD & 67 & F \\
D7 & DCD & 21 & F & D24 & DBD & 75 & M \\
D8 & DCD & 20 & M & D25 & DBD & 51 & M \\
D9 & DBD & 48 & F & D26 & DBD & 47 & M \\
D10 & DCD & 67 & M & D27 & DBD & 79 & F \\
D11 & DBD & 52 & F & D28 & DCD & 30 & M \\
D12 & DCD & 67 & M & D29 & DCD & 44 & M \\
D13 & DBD & 25 & M & D30 & DBD & 53 & F \\
D14 & DBD & 69 & F & D31 & DCD & 69 & M \\
D15 & DBD & 24 & M & D32 & DCD & 58 & M \\
D16 & DCD & 63 & M & D33 & DBD & 69 & M \\
D17 & DCD & 67 & F & & & & \\
\hline
\end{tabular}

$\mathrm{DBD}=$ donation after brainstem death $\mathrm{DCD}=$ donation after circulatory death $\mathrm{M}=$ male $\mathrm{F}=$ female 


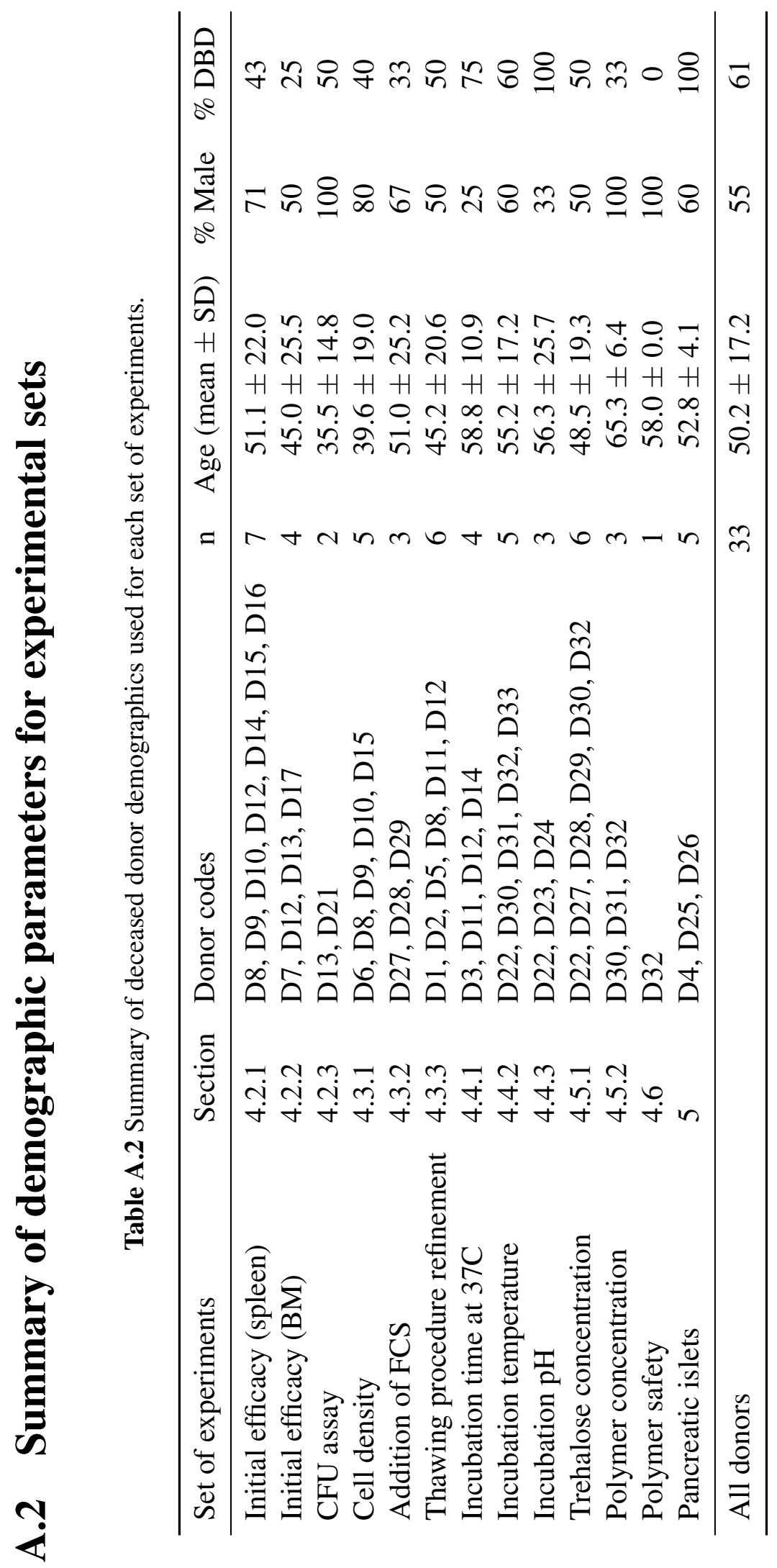




\section{A.3 Human islet preparation characteristics}

Table A.3 Clinical and islet isolation information about human islet preparations

\begin{tabular}{|c|c|c|c|}
\hline \multirow[t]{2}{*}{ Characteristic } & \multicolumn{3}{|c|}{ Islet preparation } \\
\hline & D4 & D25 & D26 \\
\hline \multicolumn{4}{|l|}{ Donor demographics } \\
\hline Age (years) & 55 & 51 & 47 \\
\hline $\operatorname{Sex}(M / F)$ & $\mathrm{F}$ & M & M \\
\hline $\mathrm{BMI}\left(\mathrm{kg} / \mathrm{m}^{2}\right)$ & 27 & 27 & 29 \\
\hline Ethnicity & white & white & white \\
\hline $\mathrm{HbA}_{1 \mathrm{c}}$ & $\mathrm{n} / \mathrm{a}$ & $\mathrm{n} / \mathrm{a}$ & $\mathrm{n} / \mathrm{a}$ \\
\hline Cause of death & $\begin{array}{c}\text { intracranial } \\
\text { haemorrhage }\end{array}$ & $\begin{array}{l}\text { intracranial } \\
\text { thrombosis }\end{array}$ & $\begin{array}{c}\text { intracranial - } \\
\text { unclassified }\end{array}$ \\
\hline Diabetes $(\mathrm{Y} / \mathrm{N})$ & $\mathrm{N}$ & $\mathrm{N}$ & $\mathrm{N}$ \\
\hline \multicolumn{4}{|l|}{ Pancreas } \\
\hline Source & Kirkcaldy & Hull & Newport \\
\hline Warm ischaemia time (h) & $7 \mathrm{~h} 2 \mathrm{~min}$ & $2 \mathrm{~h} 12 \mathrm{~min}$ & $1 \mathrm{~h} 3 \mathrm{~min}$ \\
\hline Cold ischaemia time $(\mathrm{h})$ & ND & 14 h 9 min & $11 \mathrm{~h} 24 \mathrm{~min}$ \\
\hline \multicolumn{4}{|l|}{ Islet handling and use } \\
\hline Isolation centre & Edinburgh & Edinburgh & Edinburgh \\
\hline Estimated purity (\%) & $90 \%$ & $54 \%$ & $49 \%$ \\
\hline Estimated viability (\%) & $85 \%$ & $\mathrm{n} / \mathrm{a}$ & $\mathrm{n} / \mathrm{a}$ \\
\hline Total culture time (h) & $70 \mathrm{~h}$ & $45 \mathrm{~h}$ & $70 \mathrm{~h}$ \\
\hline Used in Chapter & 2.6.1, 2.6.2 & $\begin{array}{c}5.2,5.3 .1,5.3 .2 \\
5.3 .3,5.4\end{array}$ & $5.2,5.4$ \\
\hline
\end{tabular}

This table adapted from Hart and Powers [236]. n/a = data not available 



\section{Appendix B}

\section{Flow cytometry panels and gating strategy}

Several antibody panels were used as part of this project and their development and justification of the changes are described below together with outline of the gating strategy. The markers of interest included viability and apoptosis stains, as well as CD3 for T cells, CD19 for B cells and CD34 for pooled haematopoietic progenitors.

All panels used for evaluation of pre-freeze or post-thaw viability of mononuclear cells contain the combination of Syto13 and viability dye (7-AAD or eFluor780). This combination ensured that debris can be excluded based on fluorescence, rather than less reliable light scatter characteristics [88].

In order to better identify progenitor population, additional markers were added to the first trial antibody panel (A1, Table B.1). Since haematopoietic progenitors are negative for the lineage markers, I used antibodies against CD3 and CD19 conjugated to a nearly identical fluorochromes (therefore detected at the same wavelength) and excluded these from the analysis. In addition, anti-CD133 antibody was used to help identify the progenitor population as is it another marker expressed by subset of progenitors [237]. The target population was Syto13 ${ }^{+}$, eFluor $780^{-} \mathrm{CD}^{+} 5^{+}, \mathrm{CD} 3^{-}, \mathrm{CD}^{-} 9^{-}, \mathrm{SSC}^{\text {low }}, \mathrm{CD} 34^{+}$. The gating strategy used for the A1 panel is shown in Figure B.1A.

After performing the first experiments, the panel was optimised by substituting the CD3 and CD19 antibodies for a more complex cocktail of lineage antibodies, containing CD2, CD3, CD14, CD16, CD19, CD56 and CD235a, all conjugated to APC fluorochrome. The anti-CD45 antibody was excluded from the panel since virtually all the detected alive nucleated cells were positive for this marker. Additionally, the anti-CD133 antibody was eliminated from the new panel because $\mathrm{CD} 34^{+}$population was easy to identify. The gating 

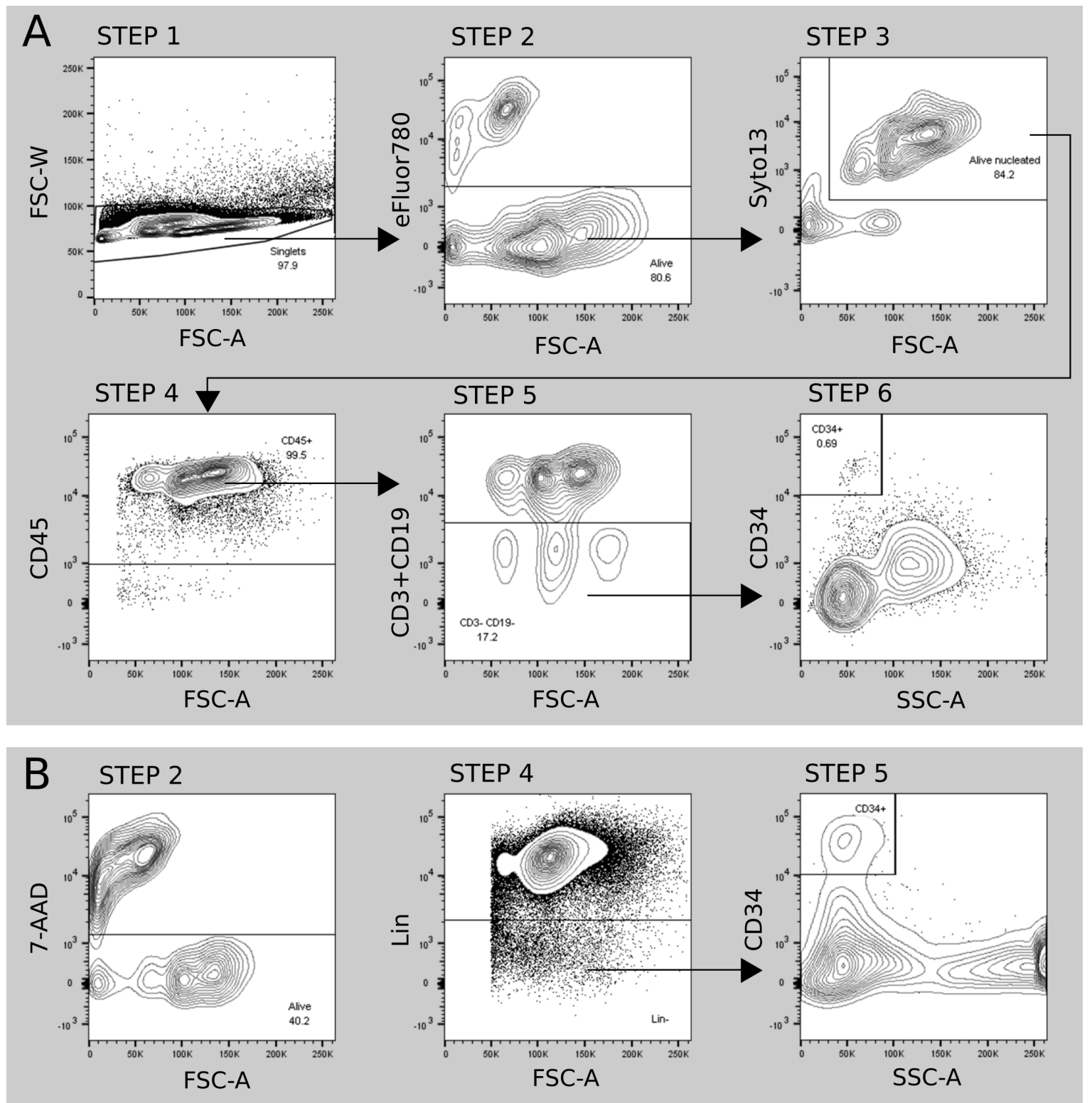

Fig. B.1 Gating strategies for enumeration of haematopoietic progenitor cells. (A) Gating strategy for the A1 panel (Table B.1). In Step 1, multiplets are excluded from the analysis. Step 2 is exclusion of dead cells using the gate on eFluor 780 positive cells, followed by selection of Syto13 positive nucleated cells in Step 3. In Step 4, cells positive for CD45 are selected. Exclusion of T cell and B cells from the analysis using the CD3 and CD19 markers is shown in Step 5. Finally, haematopoietic progenitors are selected as $\mathrm{CD}^{+} 4^{+} \mathrm{SSC}^{\text {low }}$ cells in Step 6. (B) Gating strategy for the B1 panel (Table B.1). Steps 1 and 3 are the same as in the previous strategy. In Step 2, 7-AAD viability dye is used for exclusion of dead cells instead of eFluor 780. Following Step 3, lineage negative cells are selected from the population of alive nucleated cells and finally, haematopoietic progenitors are identified as the $\mathrm{CD} 34^{+} \mathrm{SSC}^{\text {low }}$ cell subset. FSC-W $=$ forward scatter width, FSC-A = forward scatter area, $\mathrm{SSC}=$ side scatter, 7-AAD $=7$ - aminoactinomycin, $\mathrm{Lin}=$ lineage markers . 
strategy for the resulting panel B1 (Table B.1) is shown at Figure B.1B, identifying the target population of 7-AAD ${ }^{-}$, Syto $13^{+}, \mathrm{Lin}^{-}, \mathrm{SSC}^{\text {low }}, \mathrm{CD} 34^{+}$cells.

Simplified panel was also developed for experiments with limited amount of cells which combined viability and apoptosis staining, CD34, CD3 and CD19 in one panel (Panel C, Table B.1). This panel did not use the lineage cocktail but contained CD3 and CD19 which were not only used to quantify $\mathrm{T}$ and $\mathrm{B}$ cells but also to exclude lineage committed cells before identification of progenitors by the CD34 marker.

Last panel (Panel D, Table B.1) was used for flow cytometry of cells collected at the end of the CFU assay and was used for identification of myeloid and erythroid cell types.

The freezing process has been previously shown to be less damaging to haematopoietic progenitors than other cell subsets [63]. This is consistent with data from these experiments: the frequencies of haematopoietic progenitors were typically higher in conditions which had low overall viability of the mixed cell population (Figure B.2). To better asses the suitability of cryoprotectant for preservation of $\mathrm{CD}_{3} 4^{+}$cells, the absolute number of $\mathrm{CD} 34^{+}$cells after cryopreservation will be compared to that of fresh sample instead of simply comparing the frequencies of $\mathrm{CD} 34^{+}$cells in the total population.
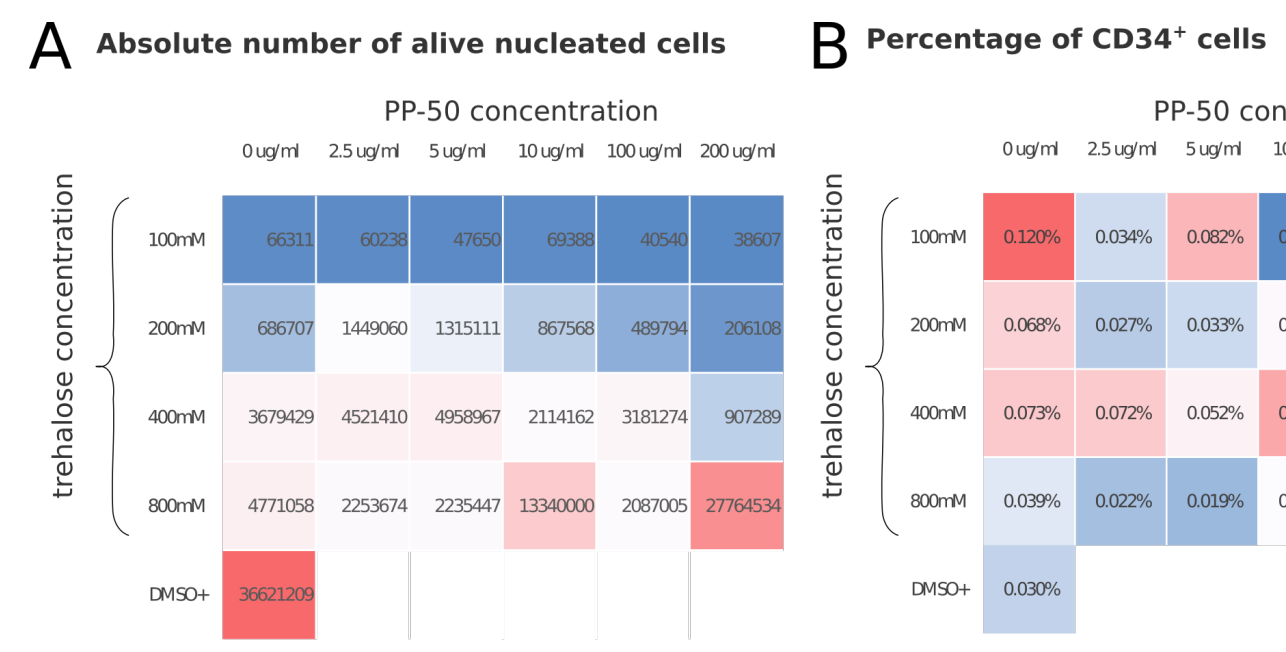

minimum

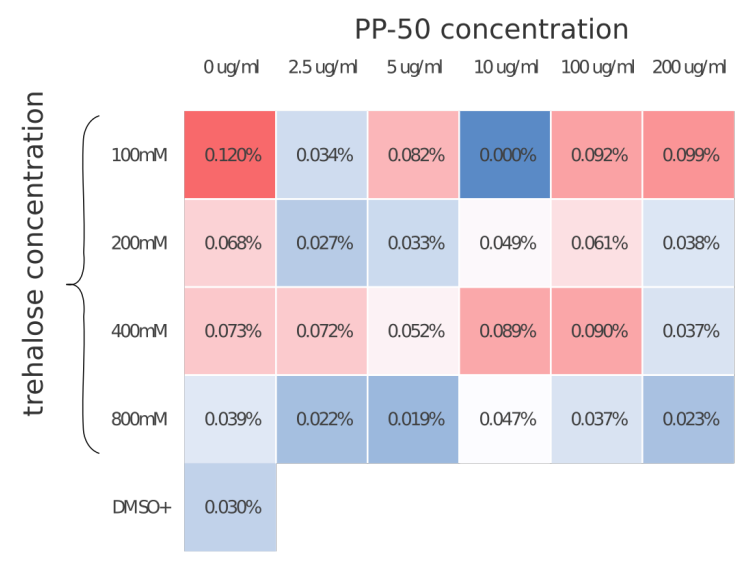

maximum

Fig. B.2 Comparison of cryopreservation protocols efficacy and frequencies of CD34 ${ }^{+}$cells. (A) Absolute cell count of alive nucleated cells determined by flow cytometry analysis in BD Trucount ${ }^{\mathrm{TM}}$ tubes for different cryopreservation conditions. (B) Frequency of CD34 ${ }^{+}$cells out of alive nucleated cell subset for different cryopreservation conditions. The colour coding of all the heat maps follows the scale on the bottom of this figure with the minimum value coded by blue and maximum value coded by red. 


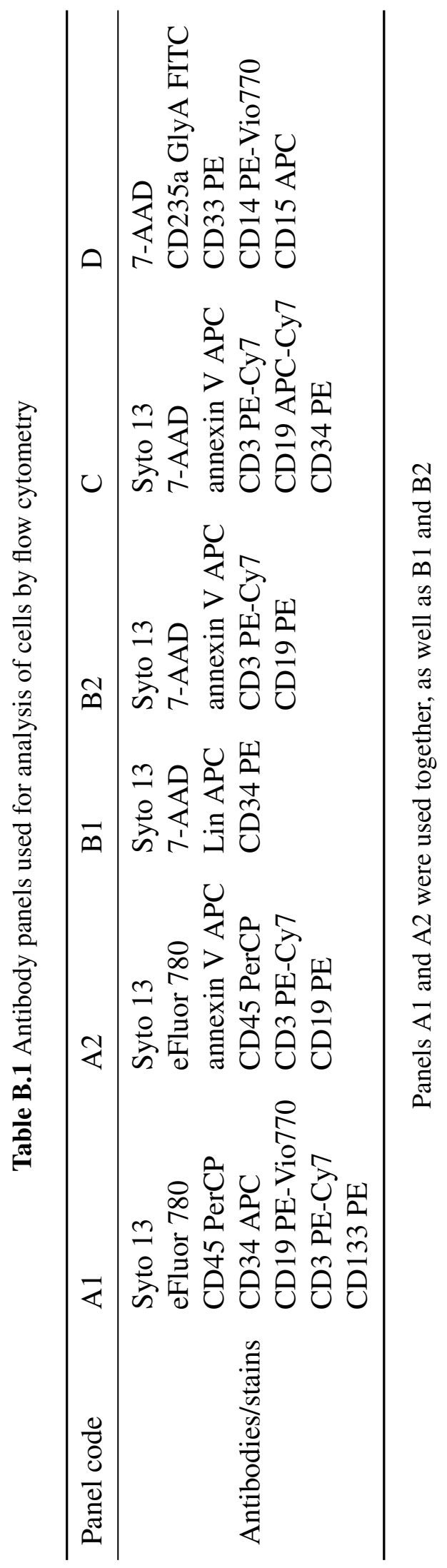




\section{Appendix C}

\section{Efficacy of permeabilising polymers in islet cryopreservation}

\section{C.1 Toxicity of polymers for pancreatic islets}

In Chapter 4, PP-50 was shown to lead to a decrease in viability of human splenocytes incubated with polymer concentrations over $200 \mu \mathrm{g} / \mathrm{mL}$. To select a safe concentration of polymer for pancreatic islets, similar toxicity assays were performed.

Mouse pancreatic islets were incubated with $0-800 \mu \mathrm{g} / \mathrm{mL}$ of PP-50 and PP-75 for 2, 24 or $48 \mathrm{~h}$ at standard culture conditions. At the end of the incubation time, viability of all islets was measured using FDA/PI staining. No decrease in viability was observed over $48 \mathrm{~h}$ period in all concentrations tested for both polymers (Figures C.1A and B).

However, it is possible that the toxicity could only be detectable if trehalose is also present. The experiments were therefore repeated using $100 \mu \mathrm{g} / \mathrm{mL}$ of PP-50 or PP-75 and range of trehalose concentrations $(0-800 \mathrm{mM})$. Again, no decrease in viability was observed with the non-toxic trehalose concentrations (Figures C.1C and D). The statistically significant viability decrease seen in $400 \mathrm{mM}$ and $800 \mathrm{mM}$ trehalose with polymers was mainly caused by trehalose, as has been shown in Section 5.2.2 of Chapter 5 .

Using one human islet donor, the toxicity test was repeated for selected conditions (200 mM trehalose, $100 \mu \mathrm{g} / \mathrm{mL}$ of PP-50 and PP-75 or their combinations). Again, viability was measured at $0 \mathrm{~h}, 2 \mathrm{~h}, 24 \mathrm{~h}$ and $48 \mathrm{~h}$ of incubation by FDA/PI staining. This data from human islets confirmed the findings from the mouse model. Even long-term incubation of islets with PP-50 or PP-75 at this concentration did not result in a decrease in viability. They are also safe to use for short incubation times in combination with $200 \mathrm{mM}$ trehalose (Figure C.1E). 

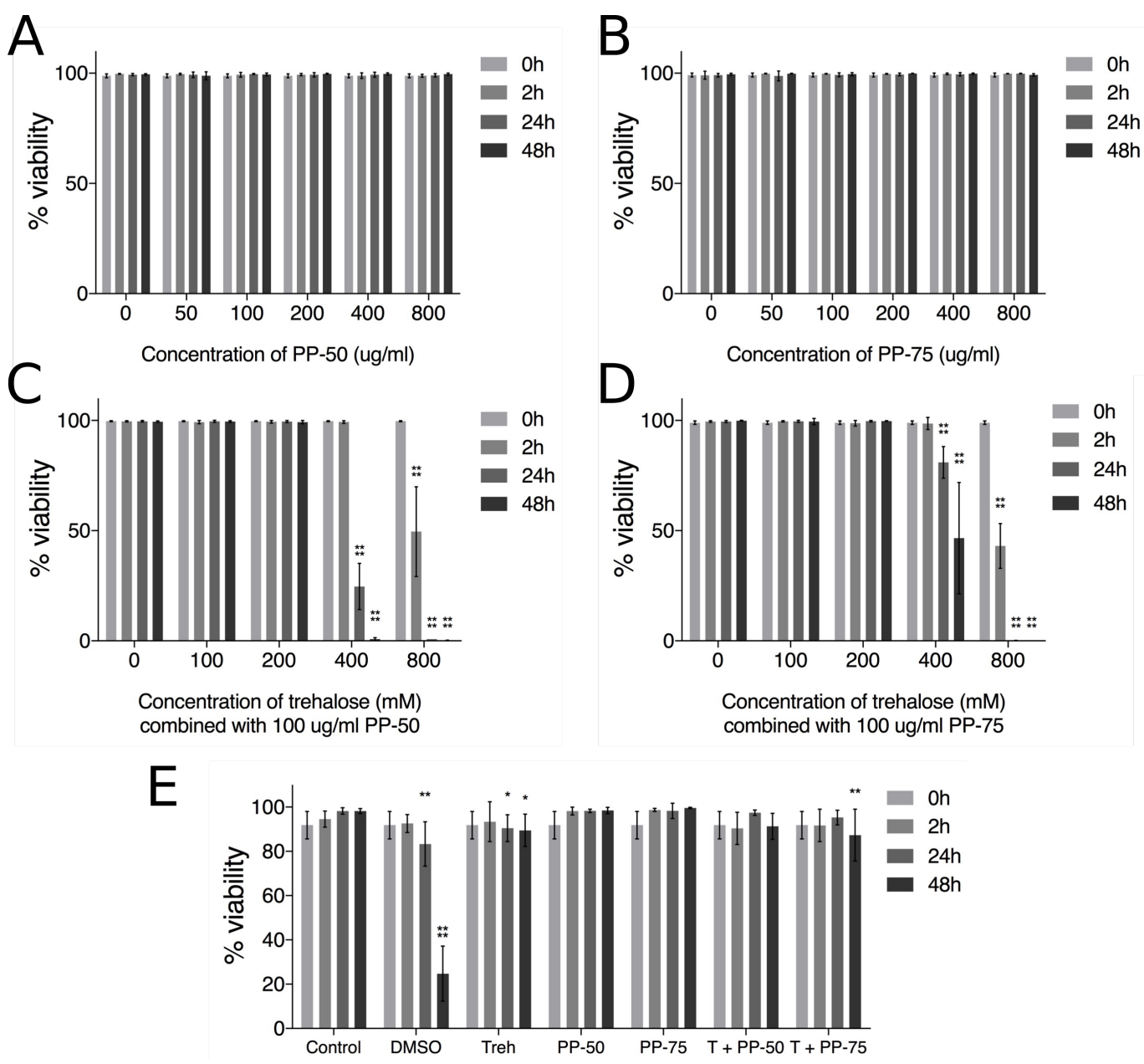

Fig. C.1 Toxicity of PP-50 and PP-75 for mouse and human pancreatic islets. (A) Viability of mouse pancreatic islets after incubation with different concentrations of PP-50 $(0-800 \mu \mathrm{g} / \mathrm{mL} ;$ mean $\pm \mathrm{SD}$ of one experiments shown, viability of each condition calculated using $6-13$ islets per experiment). (B) Viability of mouse pancreatic islets after incubation with different concentrations of PP-75 (0 $800 \mu \mathrm{g} / \mathrm{mL}$; mean \pm SD of one experiments shown, viability of each condition calculated using $7-13$ islets per experiment). (C) Viability of mouse pancreatic islets after incubation with $100 \mu \mathrm{g} / \mathrm{mL}$ PP-50 and different concentrations of trehalose $(0-800 \mathrm{mM}$; mean \pm SD of one experiments shown, viability of each condition calculated using $1-13$ islets per experiment). (D) Viability of mouse pancreatic islets after incubation with $100 \mu \mathrm{g} / \mathrm{mL}$ PP-75 and different concentrations of trehalose $(0-800 \mathrm{mM}$; mean \pm SD of one experiments shown, viability of each condition calculated using $2-13$ islets per experiment). (E) Viability of human pancreatic islets after incubation with $2 \mathrm{M}$ DMSO, $200 \mathrm{mM}$ trehalose, $100 \mu \mathrm{g} / \mathrm{mL}$ PP-50 or PP-75 and their combinations; mean \pm SD of one experiments shown, viability of each condition calculated using 3-13 islets per experiment). All groups were compared to control $(0 \mu \mathrm{g} / \mathrm{mL}, 0 \mathrm{mM}$ or Control) by two-way ANOVA followed by Dunnett test to calculate p-values. Significant differences are marked by asterisks: $* \mathrm{p} \leq 0.05, * * \mathrm{p} \leq 0.01 ; * * * \mathrm{p} \leq 0.001$; $* * * * \mathrm{p} \leq 0.0001$. 


\section{C.2 Polymer attachment to cell membrane}

The attachment of the polymer to the cell membrane is necessary to facilitate loading of extracellular molecules such as trehalose into the intracellular space. A fluorescent polymer was therefore synthesised by conjugating AlexaFluor 647 (AF647) to PP-50 and used to investigate its localisation in islet cells after incubation.

Whole or dissociated mouse pancreatic islets were incubated with fluorescent PP-50 for $4-24 \mathrm{~h}$. At the end of the incubation time, the islets/single cells were stained with Hoechst 33342 nuclear dye and PI and imaged on a confocal microscope.

In both dissociated islet cells and whole islets experiments, fluorescent PP-50 only entered selected cells while most did not have any AF647-PP-50 staining within the cells or on their surface. Interestingly, the cells which contained AF647-PP-50 also stained positively for propidium iodide which normally stains only dead cells (Figures C.2A and B). The experiment was repeated and FDA staining was added to determine viability of the loaded cells are viable or not. In Figure C.2C, both cells which stained positively for AF647-PP-50 and PI lacked FDA staining, which is consistent with the conclusion that these cells are no longer viable.

To fully understand the result of this experiment, it is important to consider the mechanism of viability staining with FDA and PI. PI is a membrane impermeable dye binding nuclear acids. It can only enter cells without an intact membrane, a marker typical for dead cells. The mechanism of fluorescein diacetate (FDA) staining uses two characteristics of live cells: active enzymes in the cytoplasm to cleave non-fluorescent FDA to produce fluorescent FITC molecule, and membrane integrity of live cells which ensures that the FITC remains locked in the cytoplasm and does not leak outside of the cells.

Two hypotheses could be postulated to explain the result observed in these experiments:

1. The polymer is only present in dead cells

- The polymer only enters dead cells OR

- The polymer kills the cells as it enters some of them.

2. The presence of polymer leads to the staining of live cells by PI

- Polymer increases fluidity of the membrane, allowing PI to enter the cells AND

- Polymer increases fluidity of the membrane so cleaved FITC leaks outside and does not accumulate in cytoplasm in detectable levels.

Of note, PP-50 appears to be accumulating in the cytoplasm since the staining is stronger than in the extracellular space. This observation is contrary to hypothesis 2 above and 

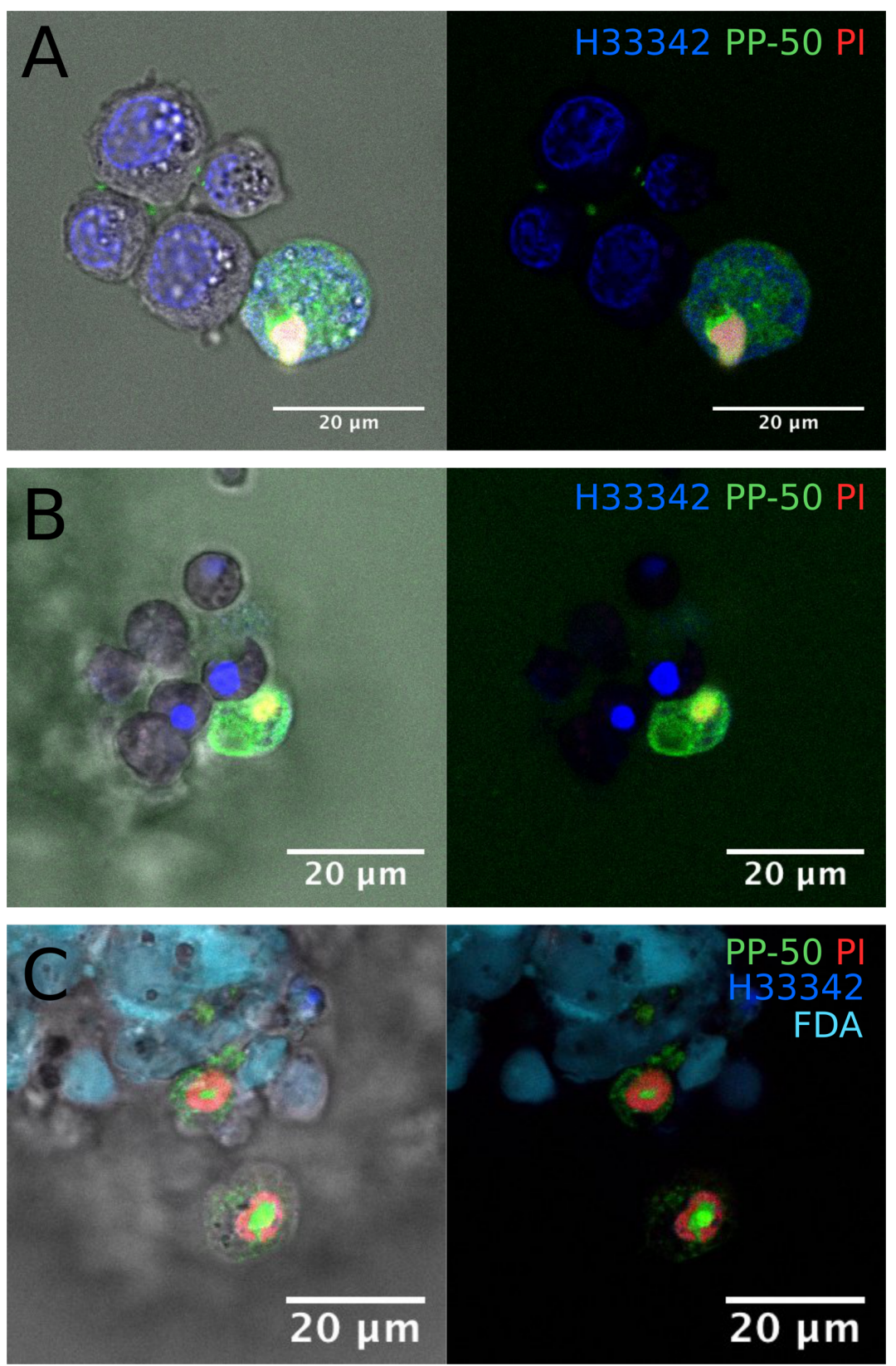

Fig. C.2 Attachment of fluorescent polymer to pancreatic islet cells. All images taken by a confocal microscope. (A) Dissociated mouse pancreatic islet cells incubated with AF647-PP50 for $4 \mathrm{~h}$ and subsequently stained with H33342 and PI. (B) Whole mouse pancreatic islet cells incubated with AF647-PP50 for $4 \mathrm{~h}$ and subsequently stained with H33342 and PI. (C) Whole mouse pancreatic islet cells incubated with AF647-PP50 for $24 \mathrm{~h}$ and subsequently stained with FDA, H33342 and PI. AF647 = AlexaFluor 647; FDA = fluorescein diacetate; H33342 = Hoechst 33342; PI = propidium iodide. 
suggests the polymer accumulated in dead cells. Nonetheless, FITC has very different electrochemical characteristics from the fluorescent polymer and it is theoretically possible for only one to leak out from the intracellular space. Nevertheless, uncontrolled leakage of any molecules from cells caused by PP-50 is a safety concern. If the cells are indeed dead, it is not clear whether they were already dead at the start of the experiment or died as a consequence of the polymer entrance. This causality could be further investigated using time-lapse imaging.

Irrespective of whether the polymer accumulates in live or dead cells, it is important to note that it selectively enters only some, and not all, cells. The cause of this variation is not clear (unless some cells are indeed dead from the beginning of the experiment and selectively accumulate the polymer). Nonetheless, these results suggest that polymers would not be suitable for safe intracellular intracellular loading of trehalose into all cells. A key limitation of this experiment is that he characteristics of the polymer may have changed by the conjugation to the fluorochrome.

A polymer with the same label was used to study attachment of PP-50 in SAOS-2 cells by Mercado et al. [146]. In this publication, it was shown that PP-50 enabled labeled trehalose to enter cells without affecting the viability of cells (measured by PI). In addition, AF647-PP-50 was detected in most cells on the presented images. Although the authors did not include appropriate controls to show that PI staining was working, this result contradicts what was found here for pancreatic islet cells. SAOS-2 is a cell line consisting of single cell type and it is expected that most cells would be viable (since all non-viable cells would detach from the surface and be washed off). However, pancreatic islets consist of many cell types with different characteristics and dead cells would remain within the sample since they are analysed in suspension culture. These and other differences between the cell types could explain the discordant findings in these two studies.

\section{C.3 Polymer efficacy in cryopreservation}

To study how addition of polymer affects the cryopreservation with trehalose, experiments were conducted using trehalose alone and in the newly developed protocol (DT6h).

First, mouse pancreatic islets were cryopreserved with $200 \mathrm{mM}$ trehalose $\pm 100 \mu \mathrm{g} / \mathrm{mL}$ PP-50/PP-75 incubated for 2 or $24 \mathrm{~h}$ or without incubation. Consistent with all other results, the viabilities obtained in cryopreservation with trehalose alone were very low. The presence of either of the polymers did not affect the post-thaw viabilities (Figure C.3A). 

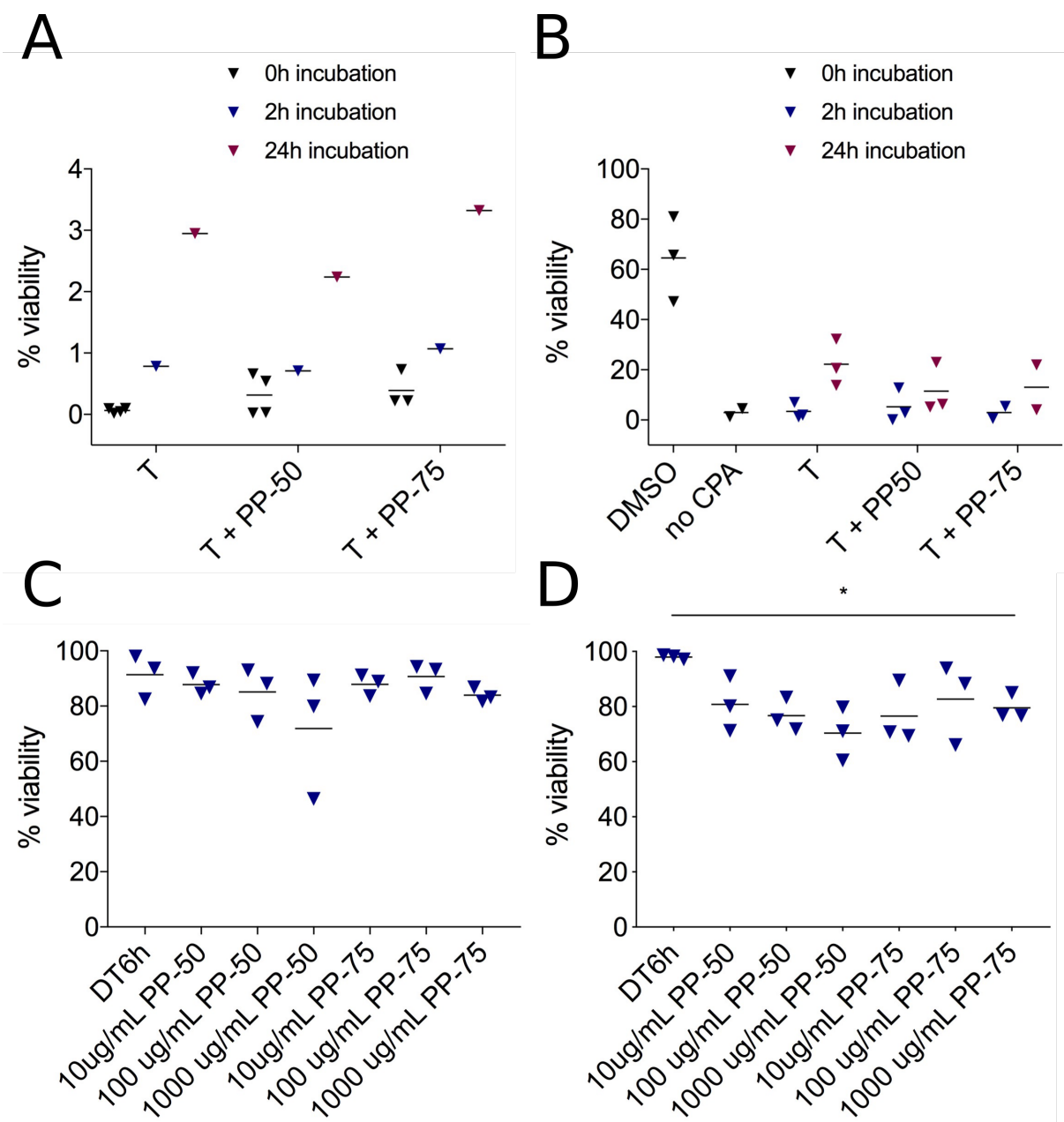

Fig. C.3 Efficacy of PP-50 and PP-75 in pancreatic islet cryopreservation. (A) Viability of mouse pancreatic islets incubated with $200 \mathrm{mM}$ trehalose $\pm 100 \mu \mathrm{g} / \mathrm{mL}$ PP-50 or PP-75 for $0 \mathrm{~h}, 2 \mathrm{~h}$ or $24 \mathrm{~h}$ measured immediately post-thaw. (B) Viability of human pancreatic islets incubated with $200 \mathrm{mM}$ trehalose $\pm 100 \mu \mathrm{g} / \mathrm{mL}$ PP-50 or PP-75 for $0 \mathrm{~h}, 2 \mathrm{~h}$ or $24 \mathrm{~h}$ measured immediately post-thaw (DMSO and no cryoprotectant controls included). (C) Viability of mouse pancreatic islets incubated with $200 \mathrm{mM}$ trehalose $\pm 100 \mu \mathrm{g} / \mathrm{mL}$ PP-50 or PP-75 for $6 \mathrm{~h}$, followed by DMSO addition and cryopreservation; measured immediately post-thaw. (D) Viability of mouse pancreatic islets incubated with $200 \mathrm{mM}$ trehalose $\pm 100 \mu \mathrm{g} / \mathrm{mL}$ PP-50 or PP-75 for $6 \mathrm{~h}$, followed by DMSO addition and cryopreservation; measured $24 \mathrm{~h}$ post-thaw. Triangles represent viabilities from individual replicates, horizontal lines represent mean. No statistical analysis was performed for (A) and(B), in (C) and (D), all groups were compared to control (DT6h) by two-way ANOVA followed by Dunnett test to calculate p-values. Significant differences $(\mathrm{p} \leq 0.05)$ are marked by asterisks. no $\mathrm{CPA}=$ nocryoprotectant; $\mathrm{T}=200 \mathrm{mM}$ trehalose, DT6h $=\mathrm{DMSO}+$ trehalose with $6 \mathrm{~h}$ incubation. 
The experiment was then repeated using human pancreatic islets. Although the post-thaw viabilities were on average higher than in mouse islets, the addition of polymer again did not lead to higher viability compared to trehalose alone (Figure C.3B).

Next, three different concentrations of PP-50 and PP-75 (10, 100 and $1000 \mu \mathrm{g} / \mathrm{mL})$ were tested in the DT6h protocol. Viability was measured immediately post-thaw and $24 \mathrm{~h}$ post thaw. Immediately post-thaw, the viabilities of the two lower concentrations of both polymers was comparable to the DT6h without any polymer. However, after $24 \mathrm{~h}$ post-thaw culture, there was a decrease in viability in all conditions where PP-50 and PP-75 were used, including significant decrease for the highest PP-75 concentration (Figure C.3C). It is noteworthy that the viability in the DT6h condition without any polymer was exceptionally high in all three replicates of the experiment; increasing this further would not be possible. However, the addition of the polymer led to a decrease in viability in all concentrations and both variants.

Taken together, this data indicate that PP-50 and PP-75 do not improve cryopreservation with neither trehalose alone nor the novel DMSO + trehalose protocol, although they were used in non-toxic concentrations. This result was consistent with the data on the splenocyte cryopreservation in Section 4.5.2 of Chapter 4. The lack of efficacy may stem from inability to enter all cells, as was shown by study of fluorescent PP-50 localisation by confocal microscopy. 



\section{Appendix D}

\section{Optimisation of in vitro functional assays}

\section{D.1 Measurements immediately versus $24 \mathrm{~h}$ post-thaw}

Pancreatic islets were cryopreserved in DMSO, no cryoprotectant agent (no CPA) or DMSO + trehalose with $3 \mathrm{~h}$ incubation time (DT3h). After thawing, ATP/ADP ratios and glucosestimulated insulin secretion (GSIS) were measured immediately or after $24 \mathrm{~h}$ post-thaw culture (fresh islet used as controls).

ATP/ADP ratios obtained from islets immediately post-thaw were very low for all cryopreservation conditions. The concentrations of ADP did not significantly differ from fresh islets (data not shown) which suggests that immediately after thawing, the ATP stores are depleted. In living and functioning islets, new ATP would be synthesised to re-establish the balance and indeed, in the successful conditions, the ATP/ADP ratio rises again after the post-thaw culture period. On the contrary, when no cryoprotectant was used (and all islets presumably died during the freezing process), new ATP is not synthesised and ATP/ADP ratio remained low (Figure D.1A). This data suggests that early measurement of ATP/ADP is less informative and it may be more appropriate to measure the ATP/ADP ratio is after a period of time needed for recovery of the ATP stores.

In the GSIS assay, a similar observation was made: none of the cryopreservation conditions resulted in islets which would respond to high levels of glucose immediately post-thaw. In addition, relatively high insulin concentrations were measured in all cryopreserved islets even in $3.3 \mathrm{mM}$ glucose concentration (Figure D.1B). This could point to the release of insulin which was already present in the islets (as opposed to newly formed and actively secreted) and is released due to cryopreservation-induced damage regardless of the glucose concentration. After $24 \mathrm{~h}$ culture period, partial recovery of islets in the successful cryopreservation conditions occurred, with decreased secretion of insulin at low glucose concentration and stimulation-dependent release of insulin in high glucose (Figure D.1C). Conversely, the 


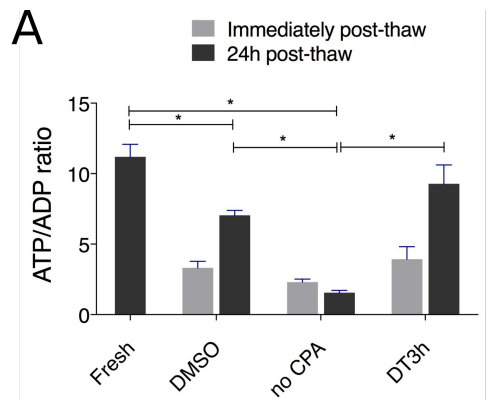

D
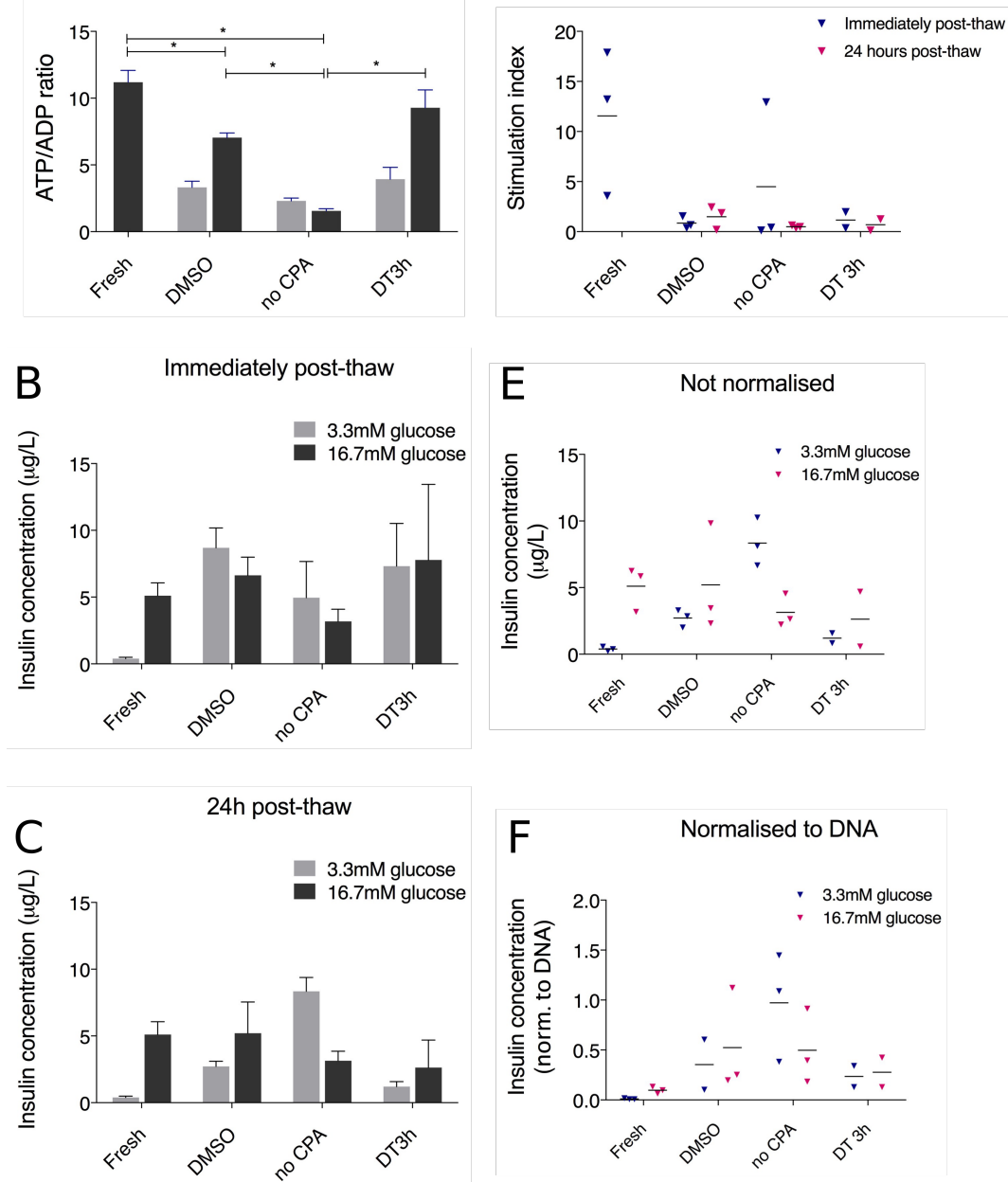

Fig. D.1 Optimisation of in vitro functional assays for pancreatic islets and their analysis. (A) ATP/ADP ratios in fresh and cryopreserved islets $(n=2-3)$. (B) Insulin secretion of fresh and cryopreserved islets stimulated with $3.3 \mathrm{mM}$ and $16.7 \mathrm{mM}$ glucose measured immediately postthaw $(\mathrm{n}=2-3)$. (C) Insulin secretion of fresh and cryopreserved islets stimulated with $3.3 \mathrm{mM}$ and $16.7 \mathrm{mM}$ glucose measured $24 \mathrm{~h}$ post-thaw $(\mathrm{n}=2-3)$. (D) Stimulation index (ratio of insulin concentration secreted in $16.7 \mathrm{mM}$ glucose and in $3.3 \mathrm{mM}$ glucose) of fresh and cryopreserved islets stimulated with $3.3 \mathrm{mM}$ and $16.7 \mathrm{mM}$ glucose $(\mathrm{n}=2-3)$. (E) Insulin secretion of fresh and cryopreserved islets stimulated with $3.3 \mathrm{mM}$ and $16.7 \mathrm{mM}$ glucose measured $24 \mathrm{~h}$ post-thaw, not normalised $(\mathrm{n}=2-3)$. (F) Insulin secretion of fresh and cryopreserved islets stimulated with $3.3 \mathrm{mM}$ and $16.7 \mathrm{mM}$ glucose measured $24 \mathrm{~h}$ post-thaw, normalised to DNA content $(\mathrm{n}=2-3)$. Bars represent mean $\pm \mathrm{SD}$, triangles represent individual measurements from each replicate, horizontal lines represent mean. Differences in (A) were measured by repeated measures one-way ANOVA, statistical differences $(\mathrm{p} \leq 0.05)$ are marked by an asterisk. no CPA $=$ no cryoprotectant; DT3h $=$ DMSO + trehalose with $3 \mathrm{~h}$ incubation time. 
unsuccessfully cryopreserved islets in no CPA condition still released high levels of insulin in $3.3 \mathrm{mM}$ glucose and decrease in insulin secretion in $16.7 \mathrm{mM}$ glucose (potentially because the stores of insulin were already depleted after the stimulation in low glucose). Because of the dysregulated release of insulin immediately after thawing, a period of recovery should therefore elapse to enable reliable measurement of post-thaw function of islets.

\section{D.2 Insulin concentration versus stimulation index}

GSIS assay data is typically presented in the literature in the form of a stimulation index. This is the ratio of the insulin concentration released in high glucose compared to low glucose concentration. Higher ratios are often associated with improved performance of islets. These ratios were calculated for the data in Figures D.1B and C and are shown in Figure D.1D.

Because the concentration of insulin released by fresh islets stimulated with $3.3 \mathrm{mM}$ glucose were very small (below 1), calculating the ratios introduced high variability in this group. In all cryopreserved conditions, the calculated stimulation index was very small both immediately and $24 \mathrm{~h}$ post-thaw. This was because insulin release in $3.3 \mathrm{mM}$ glucose was much higher than in fresh islets. In one replicate of no CPA islets measured immediately post-thaw, the ratio measured was similar to that of fresh islets, presumably because of the high variability in insulin release. It is clear, however, that this is not indicative of improved islet function in the sample because it contained only dead islets.

This data suggest that displaying the GSIS data only as stimulation indices would disregard some of the important information generated by the assay. It can be reliably used to compare islet function if the insulin release in basal glucose concentration (in this case, $3.3 \mathrm{mM}$ ) is similar for all the measured conditions. However, in the case of cryopreservation, this cannot be assumed and results should be displayed as the measured concentrations in low and high glucose concentrations.

\section{D.3 Normalisation of GSIS data}

Significant variability between the replicates was observed in the GSIS assay (Figure D.1E), possibly due to the variability in islet size. To test whether normalisation could remove some of this variability, islet pellets were kept from all GSIS samples and DNA extracted and quantified. Insulin concentrations of each sample were then normalised to its DNA content (Figure D.1F).

While the normalisation reduced variability very well for fresh islets, the variability persisted in the cryopreservation groups even after normalisation. These observations may 
be a consequence of the loss of viable islet mass during the cryopreservation and post-thaw culture. At the beginning of the assay, 8 - 10 islets per sample were randomly selected and the assay performed in triplicates for all conditions. The results in Chapter 5.5.1 demonstrated that some of the islets at $24 \mathrm{~h}$ after thawing are entirely dead and some entirely alive. It may be expected that the presence of dead islets does not increase the insulin concentration (other than perhaps through leakage) but will increase the DNA content. Because only $8-10$ islets were used for each group, this may result in significant variation between replicates. This variance would theoretically have more impact on conditions which contain large proportion of dead islets. Indeed, as shown for the no CPA condition in Figures D.1E and $\mathrm{F}$, normalisation to DNA content actually increased, rather than decreased variability.

One option to eliminate this variability would be to perform the assay only on the surviving islets. However, it is currently not possible to visually distinguish between live and dead islets at $24 \mathrm{~h}$ after thawing without viability staining. Moreover, this would provide limited information about the overall performance of the particular cryopreservation method. Another solution would be to increase the number of replicates per experimental sample with implication on the cost of the assay. From the available results, normalised and non-normalised data still provided the same conclusion about a performance of the cryopreservation condition. It is therefore not convincing that DNA normalisation adds value to the data obtained from cryopreserved pancreatic islets and for the purposes of this thesis, non-normalised data were presented.

In conclusion, the stress during cryopreservation appears to cause a transient decrease in function immediately after cryopreservation which recovers after a period of culture. Obtaining functional data from cryopreserved pancreatic islets should therefore be performed at a later time point, as demonstrated here on the examples of ATP/ADP ratio assay and GSIS assay. Furthermore, for cryopreservation data, it is necessary to display the GSIS results as the insulin concentrations in both low and high glucose concentrations instead of the stimulation index which could disregard the increase of insulin release in non-stimulated islets for unsuccessful cryopreservation conditions. Normalisation of the GSIS data to the amount of DNA introduced an additional source of variability. A future approach to reduce variability would therefore be to increase the number of replicates instead of normalisation. 


\section{Appendix E}

\section{STZ-induced diabetes in NSG mice}

\section{E.1 Dose of STZ}

There are no standardised protocols for induction of diabetes with STZ. Different research groups use different route for administration of STZ (intraperitoneal versus intravenous) or preparation of the STZ solution. Different mouse strains and genders also require very different doses of STZ for safe but effective induction of diabetes. For example, male BALB/c mice require five injections of $60-70 \mathrm{mg} / \mathrm{kg}$ of STZ while CD-1 Nude mice only $30 \mathrm{mg} / \mathrm{kg}$. Moreover, it is possible to induce diabetes with a single high dose of STZ to achieve severe injury with no remaining insulin production or use multiple smaller doses of STZ for milder diabetes with some residual $\beta$-cell function [238]. Choosing the right amount of injury is critical to ensure that it can be rescued by the intended dose of the treatment (in this case fresh and cryopreserved islets) but at the same time, the animals should not recover spontaneously. For this reason, the model using multiple low doses of STZ was chosen in this project.

Because one of the future aims of this project is to test the function of cryopreserved human islets in vivo, the choice of an immunodeficient mouse strain was important. The NSG immunodeficient mouse strain is well established and was previously used to study survival of fresh and cryopreserved mouse or human pancreatic islets and was also used for the STZ-induced diabetes experiments. However, protocols for this mouse strain have not been published and protocols for generation of STZ-induced diabetic NSG mice required optimisation.

The first dose tested was $75 \mathrm{mg} / \mathrm{kg}$ intraperitoneally for three consecutive days. This dose was used to induce diabetes in another strain of immunodeficient mice, Scid/Beige mice, in a publication by Lima et al. [239]. Using this dose, rapid onset of diabetes was observed, reaching severe levels in less than one week after the first injection. This resulted in rapid weight loss, indicating toxic effect of this high dose of STZ. The experiment had to 


\section{A 3x $75 \mathrm{mg} / \mathrm{kg} \mathrm{sTz}$}
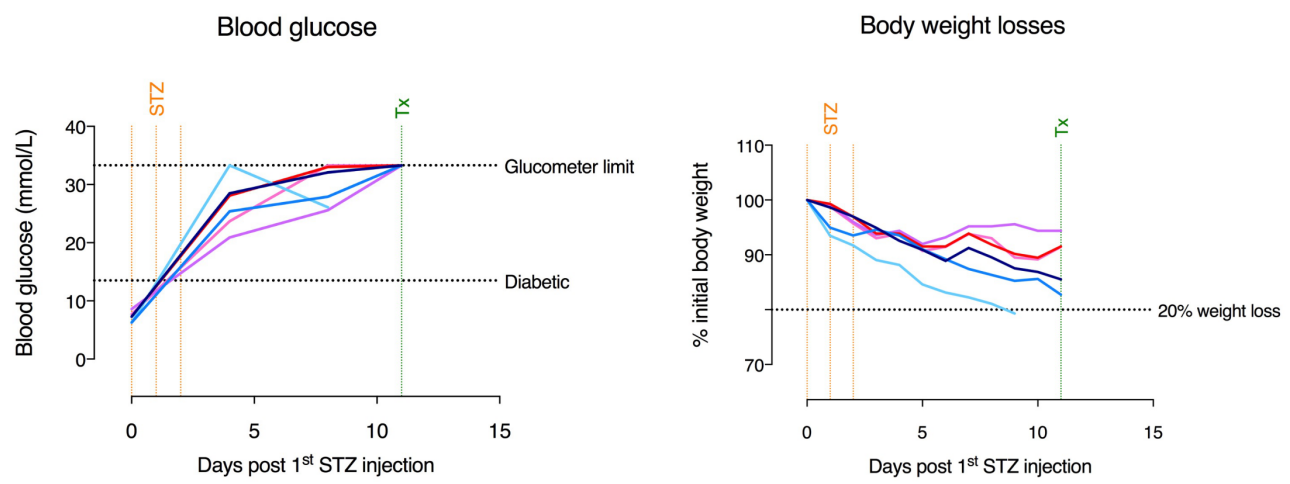

B 5x $50 \mathrm{mg} / \mathrm{kg} \mathrm{sTZ}$
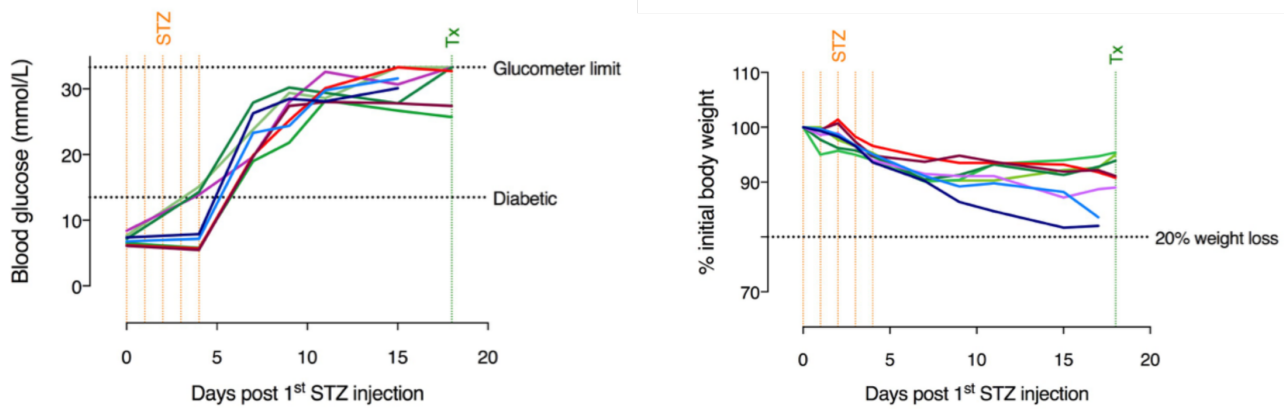

$C_{5 \times} 40 \mathrm{mg} / \mathrm{kg} \mathrm{sTZ}$
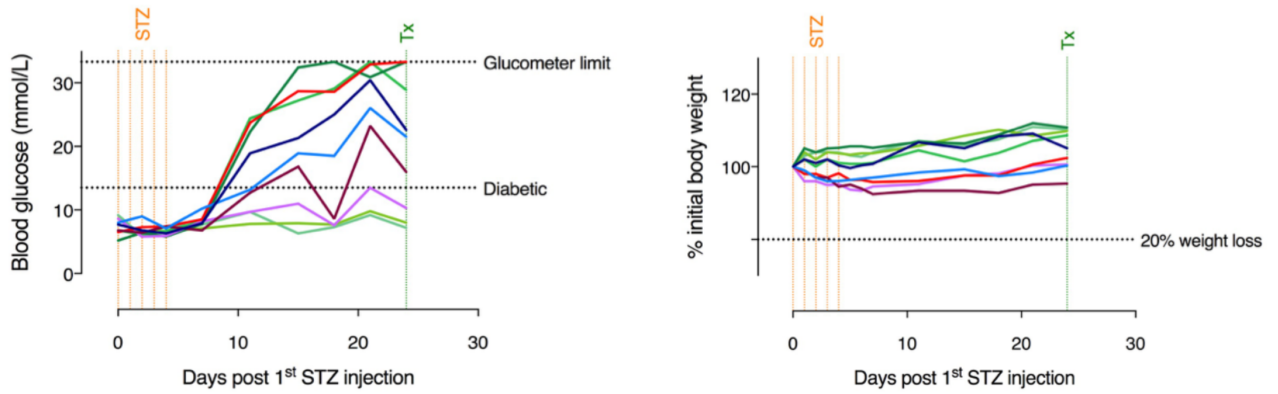

Fig. E.1 Optimisation of STZ dose in the in vivo diabetes model using NSG mice. (A) Blood glucose levels and weight losses of individual NSG mice injected with $75 \mathrm{mg} / \mathrm{kg} \mathrm{STZ} \mathrm{for} 3$ consecutive days $(\mathrm{n}=6)$. (B) Blood glucose levels and weight losses of individual NSG mice injected with $50 \mathrm{mg} / \mathrm{kg}$ STZ for 5 consecutive days $(n=8)$. (C) Blood glucose levels and weight losses of individual NSG mice injected with $40 \mathrm{mg} / \mathrm{kg} \mathrm{STZ}$ for 5 consecutive days $(\mathrm{n}=9)$. Three separate experiments are shown in figures (A), (B) and (C), all experimental animals shown in a single graph were tested concurrently. Each line connects the measured values from an individual animal. Days of STZ injections (STZ) and islet transplantations under kidney capsule (Tx) are indicated in the graphs by vertical lines. $\mathrm{STZ}=$ streptozotocin; $\mathrm{Tx}=$ transplantation. 
be terminated for two out of six mice (day 9 and 12) because the mice approached the $20 \%$ weight loss, which is an endpoint stipulated by the Home Office (Figure E.1A).

Two lower doses of STZ were therefore tested: i.p. injections of $50 \mathrm{mg} / \mathrm{kg}$ in five consecutive days and $40 \mathrm{mg} / \mathrm{kg}$ in five consecutive days. These doses work well for variety of mouse strains [238]. In NSG mice, five $50 \mathrm{mg} / \mathrm{kg}$ injection caused slower onset of diabetes but still resulted in weight loss in all of the animals (two out of eight terminated due to $20 \%$ weight loss on day 17, Figure E.1B). Five injections of $40 \mathrm{mg} / \mathrm{kg}$ did not result in any significant weight loss but there was high variability between the glucose levels in individual mice. Three out of 9 animals did not develop diabetes at all while some have reached severe levels of glucose in about two weeks after the first injection (Figure E.1C). The dose used by The Jackson Laboratory in commercially available mice is also $40 \mathrm{mg} / \mathrm{kg}$ for five consecutive days [240].

To conclude, the two higher doses tested $(75 \mathrm{mg} / \mathrm{kg}$ for 3 consecutive days and $50 \mathrm{mg} / \mathrm{kg}$ for five consecutive days intraperitoneally) caused rapid weight loss in most of the injected animals. Lower, non-toxic dose must therefore be used in further experiments. Although significant variability in glucose levels was observed with the lowest dose $(40 \mathrm{mg} / \mathrm{kg}$ for 5 consecutive days), there was no weight loss associated with this. This dose was therefore chosen for the future experiments. Experiments included larger groups of animals so that non-diabetic animals can potentially be excluded from the group before the transplantation.

\section{E.2 Dose of pancreatic islets for transplantation}

In the initial experiments, 300 fresh islets were transplanted under the kidney capsule of each diabetic NSG mice (based on a recommendation by Dr. Maja Wallberg for a minimum islet dose to reverse diabetes) but in only 3 out of 6 animals this led to a decrease in blood glucose levels after the transplantation (data not shown). The possible reasons include low dose of transplanted pancreatic islets or variations introduced by the technique of the surgeon.

Male NSG mice were next rendered diabetic using i.p. injections of $50 \mathrm{mg} / \mathrm{kg} \mathrm{STZ}$ for five consecutive days (Figure E.1B) and transplanted with 300 or 400 freshly isolated pancreatic islets from male C57BL/6JAX mice under the kidney capsule (3 mice per group). Body weight and blood glucose was measured regularly and the experiment was terminated 4 weeks after the transplantation.

The body weights and blood glucose levels were well matched in the two groups before transplantation. After transplantation, there was an increase in body weight for both groups but it was more pronounced in the group transplanted with higher dose of islets (Figure E.2A). There was high variability in the blood glucose level within the groups (Figure E.2B). 

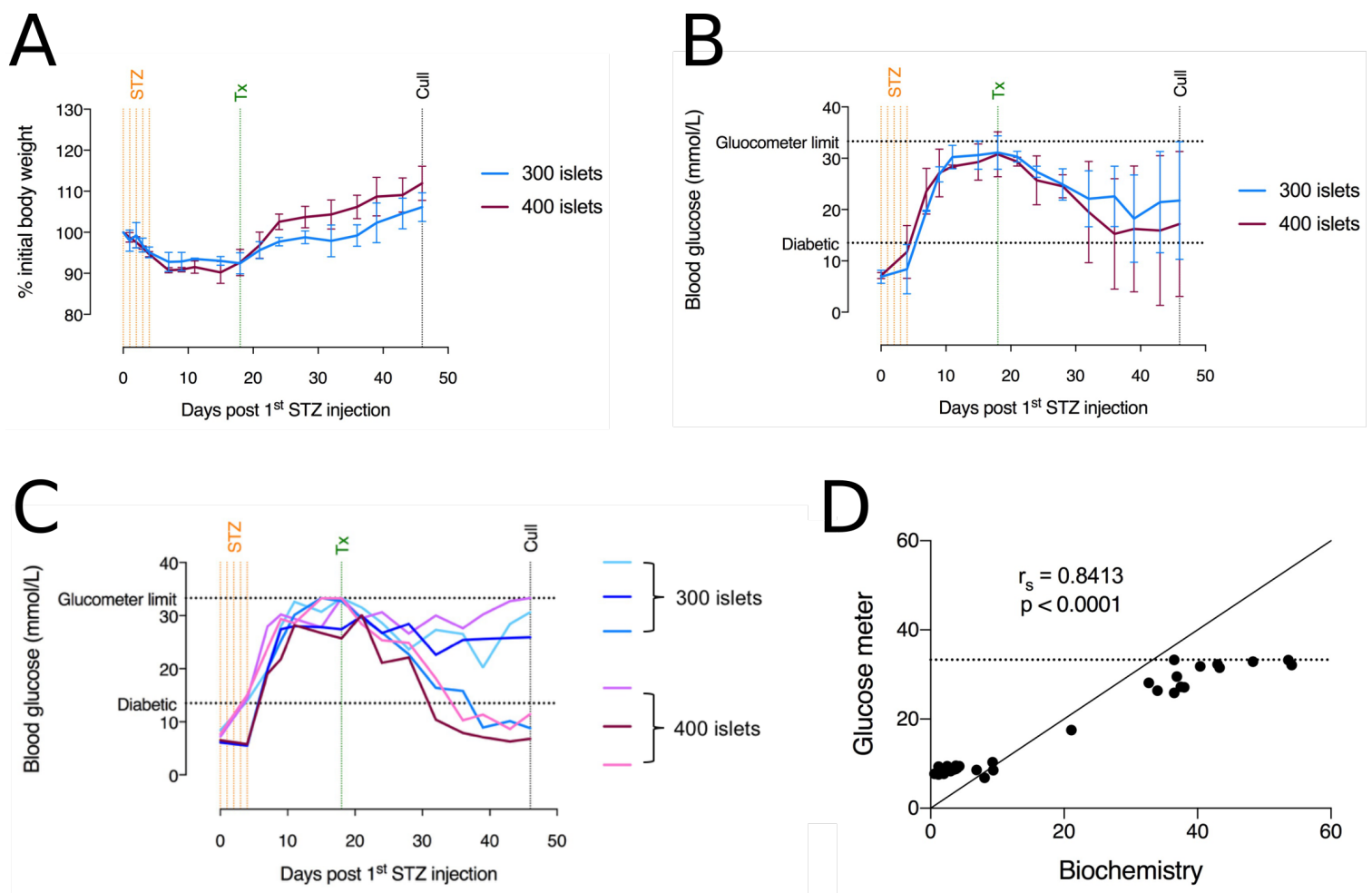

Fig. E.2 Optimisation of pancreatic islet dose for transplantation under the kidney capsule of diabetic NSG mice. (A) Average weight losses of animals injected with $50 \mathrm{mg} / \mathrm{kg} \mathrm{STZ}$ for 5 consecutive days and transplanted with 300 or 400 fresh mouse islets under the kidney capsule ( $\mathrm{n}=3$ animals per group). (B) Average blood glucose levels of animals injected with $50 \mathrm{mg} / \mathrm{kg}$ STZ for 5 consecutive days and transplanted with 300 or 400 fresh mouse islets under the kidney capsule ( $\mathrm{n}=3$ animals per group). (C) Blood glucose levels of individual NSG mice injected with $50 \mathrm{mg} / \mathrm{kg} \mathrm{STZ}$ for 5 consecutive days and transplanted with 300 or 400 fresh mouse pancreatic islets under the kidney capsule $(n=6)$. All experimental animals shown were tested concurrently. In (A) to (C), days of STZ injections (STZ), islet transplantations under kidney capsule (Tx) and the end of the experiment (Cull) are indicated in the graphs by vertical lines. STZ $=$ streptozotocin; $\mathrm{Tx}=$ transplantation. (D) Correlation between measurements from the portable glucometer and biochemical assay measured by the CBAL laboratory $(\mathrm{n}=38)$. Line of identity (slope $=1)$ is displayed on the graph (solid line), as well as the limit of the portable glucose meter (horizontal dashed line). Nonparametric Spearman's correlation coefficient $\left(r_{s}\right)$ was calculated and is displayed together with the $\mathrm{p}$-value. 
Inspection of the data from the individual animals reveals that in both groups, there were animals in which the diabetes was reversed and animals in which there was only slight (or temporary) decrease in blood glucose levels. Specifically, only one out of three animals transplanted with 300 islets and two out of three animals transplanted with 400 islets were not diabetic at the end of the experiment (Figure E.2C). When the transplanted kidneys were excised at the end of the experiment, very little or no visible islets were present in the graft under the kidney capsule of mice in which the diabetes was not reversed (data not shown).

The animals did not differ significantly in age, weight and were not housed in different cages. The animals were all transplanted by one surgeon on the same day within the minimal time possible $(2 \mathrm{~h})$. No technical errors or differences between individual transplantations were observed and the islets were clearly visible under the kidney capsule at the time of injection in all animals.

In conclusion, there is inherent biological variation in these experiments, the cause of which was not identified. Increasing the dose of fresh pancreatic islets from 300 to 400 per animal still did not guarantee the reversal of diabetes in all animals.

\section{E.3 Validation of the portable glucometer}

A comprehensive testing or glucometers was conducted by Togashi et al. where they reported that most glucometers record higher values than the biochemical test but the values generally correlated with the biochemistry results [241]. To evaluate the particular model of glucometer used in this project (iHealth Smart), multiple blood serum samples were taken over the course of the in vivo experiments at the same time as measurements by the portable glucose meter and sent for a biochemical analysis to the CBAL laboratory. Measurements which were over the limit of the portable glucose meter $(>33.3 \mathrm{mmol} / \mathrm{L})$ were excluded from the analysis and data from the matched measurements were then correlated.

The result suggested a strong correlation of the two measurements ( $\mathrm{p} \leq 0.0001)$, although low glucose concentrations were slightly overestimated and high values slightly underestimated by the iHealth Smart glucose meter, as can be seen in Figure E.2D. The biggest error of the glucose meter was observed for values close to the limit $(33.3 \mathrm{mmol} / \mathrm{L})$. In the available publication, only 1 out of 11 glucometers recorder lower values than biochemical test, the rest showed higher values, across the range of glucose concentrations [241]. 